\title{
The Epidemiology of Parkinsonism
}

Anette Schrag

Thesis submitted in fulfilment of the requirements for the degree of

Doctor of Philosophy

2000

Department of Clinical Neurology

Institute of Neurology

University of London 
ProQuest Number: U642213

All rights reserved

INFORMATION TO ALL USERS

The quality of this reproduction is dependent upon the quality of the copy submitted.

In the unlikely event that the author did not send a complete manuscript and there are missing pages, these will be noted. Also, if material had to be removed, a note will indicate the deletion.

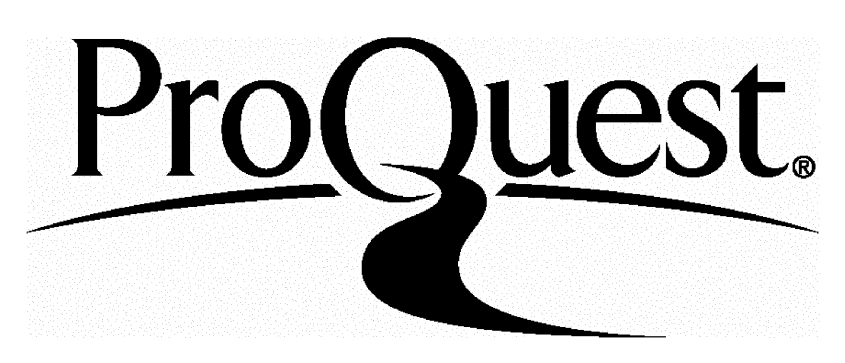

ProQuest U642213

Published by ProQuest LLC(2015). Copyright of the Dissertation is held by the Author.

All rights reserved.

This work is protected against unauthorized copying under Title 17, United States Code. Microform Edition (c) ProQuest LLC.

\author{
ProQuest LLC \\ 789 East Eisenhower Parkway \\ P.O. Box 1346 \\ Ann Arbor, MI 48106-1346
}




\begin{abstract}
This thesis is concerned with the descriptive epidemiology of parkinsonism with a particular emphasis on the differentiation of parkinsonian disorders. The areas investigated were the population prevalence of different parkinsonian disorders, the accuracy of their diagnosis in the community, and the clinical characteristics and natural history of Parkinson's disease (PD) in a population-based sample, including quality of life (QoL).
\end{abstract}

The age-adjusted prevalence rates of MSA was 4.4, of PSP 6.4, and of PD 168 per 100,000 . At least $15 \%$ of patients with a diagnosis of PD in the general population were misdiagnosed, and, conversely, approximately $20 \%$ of patients were unrecognised to have PD.

The most disabling problems in the population of patients with PD in the community were disease severity, depression and dementia. Unfavourable prognostic factors were older age at onset and development of dementia. The rates of motor complications in PD increased with disease duration and severity, but were also related to dose of levodopa and duration of levodopa treatment. Depression in patients with PD was associated with advancing disease severity and disability. However, it was also strongly influenced by the patients' own perceptions of their handicap rather than being simply the result of objective impairment.

This study also found evidence for the existence of different subtypes of PD. Factors associated with a faster rate of progression but a lower rate of motor fluctuations were older age at onset, older current age and presence of dementia. $\mathrm{PD}$ in this community-based sample of patients interfered with various aspects of QoL, particularly with those related to physical and social functioning, whereby depression, disability, postural instability and cognitive impairment had the greatest influence on QoL. The generic instrument EQ-5D was demonstrated to be a feasible and valid instrument to measure QoL in PD that reflects severity and complications of disease. 


\section{LIST OF CONTENTS}

$\begin{array}{lr}\text { List of tables } & 12\end{array}$

$\begin{array}{ll}\text { List of figures } & 14\end{array}$

List of abbreviations 15

$\begin{array}{ll}\text { Acknowledgements } & 16\end{array}$

\section{Chapter 1: Introduction}

1.1. The subtypes of parkinsonism and their diagnosis 18

$\begin{array}{lll}\text { 1.2. } & \text { Epidemiology }\end{array}$

1.3. Descriptive epidemiology of parkinsonism 27

Chapter 2: Aims of the study

2. 1. The prevalence and incidence of parkinsonian disorders 31

in the population

2.2. Accuracy of diagnosis 31

2.3. The clinical features and natural history of parkinsonian 31 disorders in a population-based sample

2.4. Quality of life in Parkinson's disease 33

Chapter 3: Methodology

3.1. Patient ascertainment. 33

3.1.1. Linkage Scheme with general practices 33

3.1.2. Patient searches 34

3.1.2.1. Stage I 34

3.1.2.2. Stage II 36

3.1.2.2.1. Inclusion criteria 36

3.1.2.2.2. Exclusion criteria 36

3.1.2.3. Stage III 37

$\begin{array}{lll}\text { 3.1.3. } & \text { Patient contact }\end{array}$ 
3.2. Patient assessment 38

3.2.1. Diagnostic visit $\quad 38$

3.2.1.1. Diagnosis $\quad 39$

3.2.1.2. Diagnostic criteria 40

3.2.1.3. Assessment of depression and quality of life 40

3.2.2. Follow-up 41

3.3. Data analysis 41

3.4. Approval by an Ethics Committee 41

3.5. Confidentiality and patient consent 42

Chapter 4: The population prevalence and incidence of the atypical

parkinsonian disorders progressive supranuclear palsy

and multiple system atrophy

4.1. Background 43

4.2. Method 43

4.2.1. Diagnosis 44

4.2.2. Diagnostic criteria 45

4.2.3. Data analysis 45

4.3. Results 45

4.3.1. Prevalence 46

4.3.2. Gender differences 47

4.3.3. Incidence 47

$\begin{array}{lll}\text { 4.4. Discussion } & 47\end{array}$

Chapter 5: The population prevalence of Parkinson's disease and

parkinsonism in London

5.1. Background 51

5.2. Patients and methods 51

5.2.1. Diagnostic criteria 51

5.2.2. Data analysis 52

$\begin{array}{lll}\text { 5.3. } & \text { Results } & 52\end{array}$ 
5.3.1.

Prevalence

5.3.2.

Age- and sex-specific rates

5.3.3.

Relative frequency of parkinsonian subtypes among 53

patients with parkinsonism

5.4. Discussion

5.4 .1

Comparison with findings from other UK studies

54

5.4 .2 .

Comparison with findings from other studies

world-wide

5.4 .3

Potential underascertainment

5.4.4.

Diagnostic accuracy

5.4.5.

Other causes of a parkinsonian syndrome

56

5.5 .

Conclusion

57

Chapter 6. Accuracy of the clinical diagnosis of Parkinson's disease in the community

6.1. Background 58

6.2. Methods 58

6.3. Results 58

6.3.1. Patients with a previous diagnosis of Parkinson's disease 59

6.3.2. Patients with a previous diagnosis other than 59

Parkinson's disease

6.3.3. Sensitivity, specificity and predictive value of a previous 59

diagnosis of Parkinson's disease

6.3.4. Comparison of patients in whom a diagnosis of 60

Parkinson's disease was maintained or rejected

6.3.5. Comparison of Parkinson's disease patients in whom a 61

diagnosis of Parkinson's disease was or was not previously

made 
Chapter 7: The clinical features and natural history of parkinsonian disorders

7.1. Clinical characteristics of patients with progressive supranuclear 65 palsy and multiple system atrophy

7.2. The clinical features of Parkinson's disease in a population-based 66 sample

7.2.1. Background 66

7.2.2. Methods 66

$\begin{array}{lll}\text { 7.2.3. Statistical analysis } & 66\end{array}$

$\begin{array}{lll}\text { 7.2.4. Results } & 66\end{array}$

7.2.4.1. Primary symptom at onset 67

$\begin{array}{lll}\text { 7.2.4.2. Findings } & 67\end{array}$

7.2.4.3. Disease severity 67

7.2.4.4. Treatment 67

7.2.4.5. Side effects of medication 68

7.2.4.6. Family history and socio-economic background 68

$\begin{array}{lll}\text { 7.2.4.7. Potential risk factors } & 68\end{array}$

7.2.4.8. Socioeconomic consequences of the disease 68

7.2.4.9. Factors determining disability 68

7.2.4.10. Rate of progression 69

$\begin{array}{lll}\text { 7.2.5. Discussion } & 69\end{array}$

7.2.5.1. Clinical features $\quad 69$

7.2.5.2. Treatment 70

7.2.5.3. Complications of disease and treatment 70

$\begin{array}{lll}\text { 7.2.5.4. Disability } & 71\end{array}$

$\begin{array}{lll}\text { 7.2.5.5. Family history } & 71\end{array}$

7.2.5.6. Rate of progression 72

7.2.5.7. Factors influencing disease progression 72 
7.3. Dyskinesias and motor fluctuations in Parkinson's disease $\quad 74$

$\begin{array}{lll}\text { 7.3.1. } & \text { Background } & 74\end{array}$

7.3.2. Methods 75

$\begin{array}{lll}\text { 7.3.3. Statistical analysis } & 75\end{array}$

$\begin{array}{lll}\text { 7.3.4. } & \text { Results }\end{array}$

7.3.4.1. Prevalence of motor fluctuations and dyskinesias 76

7.3.4.2. Rate of motor complications by disease duration, 76 treatment duration and Hoehn and Yahr stage

$\begin{array}{lll}\text { 7.3.4.3. Subtypes of motor fluctuations and dyskinesias } & 77\end{array}$

7.3.4.4. Time to onset of complications 77

7.3.4.5. Differences between patients with and without motor 78

fluctuations and dyskinesias

7.3.4.6. Discriminant analysis $\quad 79$

7.3.4.7. Patients without motor complications after more than 79

10 years of treatment with levodopa

$\begin{array}{lll}\text { 7.3.4.8. } & \text { Quality of life }\end{array}$

$\begin{array}{lll}\text { 7.3.5. Discussion } & 80\end{array}$

7.4. Depression in Parkinson's disease 84

7.4.1. Background $\quad 84$

$\begin{array}{lll}\text { 7.4.2. } & 84\end{array}$

7.4.3. Statistical analysis $\quad 85$

7.4.4. Results $\quad 85$

7.4.4.1. Extent of depression 85

7.4.4.2. Relation of depression to patient- and disease 85 characteristics

7.4.4.3. Relation of depression to disability 87

7.4.4.4. Relation of depression to self-rated health status 87

7.4.4.5. Multiple regression analysis $\quad 87$

7.4.4.5.1. Disease-characteristics - Degree of impairment 88

$\begin{array}{lll}\text { 7.4.4.5.2. Disability } & 88\end{array}$ 
7.4.4.5.3. Quality of life 88

7.4.4.5.4. Overall: impairment, disability and QoL 88

7.4.5. Discussion 88

7.4.5.1. Prevalence of depression in Parkinson's disease 88

7.4.5.2. Factors contributing to depression 88

7.5. The clinical subtypes of Parkinson's disease 92

$\begin{array}{lll}\text { 7.5.1. } & \text { Background } & 92\end{array}$

7.5.2. Methods 92

$\begin{array}{ll}\text { 7.5.3. Statistical analysis } & 92\end{array}$

7.5.4. Results 93

7.5.4.1. Young (<66 years) vs old age at onset ( $>66$ years) 94

7.5.4.2. Symptom at onset 94

7.5.4.3. Akinetic-rigid vs tremor-dominant Parkinson's disease 94

7.5.4.4. Slow vs fast progression 94

7.5.4.5. Young (<72 years) vs older age ( $>72$ years) 95

7.5.4.6. Gender differences 95

7.5.4.7. Family history of parkinsonism 95

7.5.4.8. Patient with and without dementia 95

7.5.4.9. Patients with and without depression 96

7.5.4.10. Patients with and without a history of hallucinations 96

7.5.4.11. Cluster analysis 96

$\begin{array}{lll}\text { 7.5.5. Discussion } & 98\end{array}$

7.6. Young onset Parkinson's disease - Clinical features, 103 and mortality

$\begin{array}{lll}\text { 7.6.1. } & 103\end{array}$

$\begin{array}{ll}\text { 7.6.2. } & 103\end{array}$

$\begin{array}{lll}\text { 7.6.3. } & 105\end{array}$

$\begin{array}{lll}\text { 7.6.3.1. Juvenile parkinsonism } & 105\end{array}$

$\begin{array}{lll}\text { 7.6.3.2. } & \text { Young onset Parkinson's disease } & 107\end{array}$ 
7.6.3.2.1.

7.6.3.2.2.

7.6.3.2.3.

7.6.3.2.4.

7.6.3.2.5.

7.6.3.3.

7.6.3.3.1.

7.6.3.3.2.

7.6.4.
Onset

107

Complications of 1-dopa treatment

107

Current state

107

Dementia

108

Mortality

109

Prospective subgroup of 60 patients

109

109

109

Young Onset Parkinson's disease

Discussion

\section{Chapter 8: Quality of life in Parkinson's disease}

8.1. Comparison of the EQ-5D, the SF 36 , two generic quality of life

measures, and the PDQ 39, a disease-specific instrument, to measure quality of life in patients with Parkinson's disease

8.1.1. Background 115

$\begin{array}{ll}\text { 8.1.2. Patients and methods } & 116\end{array}$

8.1.2.1. Qol instruments 116

$\begin{array}{lll}\text { 8.1.2.2. Statistical analysis } & 117\end{array}$

$\begin{array}{ll}\text { 8.1.3. Results } & 117\end{array}$

$\begin{array}{lll}\text { 8.1.3.1. Feasibility } & 117\end{array}$

$\begin{array}{lll}\text { 8.1.3.2. Validity } & 118\end{array}$

8.1.3.2.1. Correlation with ratings of disease severity 118

$\begin{array}{lll}\text { 8.1.3.2.1.1. EQ-5D summary index } & 118\end{array}$

$\begin{array}{lll}\text { 8.1.3.2.1.2. Visual analogue scale } & 118\end{array}$

8.1.3.2.2. Correlation with other QoL 119

8.1.3.2.3. Sensitivity to clinical features of Parkinson's disease 119

8.1.3.2.3.1. EQ-5D summary index and Visual analogue scale 119

8.1.3.2.3.2. Comparisons with other QoL measures 120

$\begin{array}{ll}\text { 8.1.4. Discussion } & 120\end{array}$

8.2. The impact of Parkinson's disease on quality of life 123 
$\begin{array}{ll}\text { 8.2.2. Patients and methods } & 123\end{array}$

$\begin{array}{ll}\text { 8.2.3. Results } & 123\end{array}$

8.2.3.1. The EQ-5D instrument 123

8.2.3.2. The Medical Outcome Study Short Form (SF 36) instrument 124

$\begin{array}{lll}\text { 8.2.3.3. The PDQ-39 instrument. } & 125\end{array}$

$\begin{array}{ll}\text { 8.2.4. Discussion } & 125\end{array}$

8.3. Factors that contribute to quality of life in patients with Parkinson's 129 disease

$\begin{array}{ll}\text { 8.3.1. Background } & 129\end{array}$

$\begin{array}{ll}\text { 8.3.2. Patients and methods } & 129\end{array}$

$\begin{array}{ll}\text { 8.3.3. Results } & 129\end{array}$

8.3.3.1. Impact of clinical features on PDQ-39 scores 129

8.3.3.2. Correlations of clinical scores with PDQ-39 130

$\begin{array}{ll}\text { 8.3.3.3. Regression analysis of PDQ } 39 \text { scores } & 130\end{array}$

$\begin{array}{ll}\text { 8.3.4. Discussion } & 140\end{array}$

Chapter 9: Summary, conclusions and implications

9.1. The population prevalence and incidence of atypical parkinsonian 135 disorders

9.2. The population prevalence of Parkinson's disease 136

9.3. Accuracy of the clinical diagnosis of Parkinson's disease in the 137 community

9.4. The clinical features of Parkinson's disease in a population-based 137 sample

9.5. Dyskinesias and motor fluctuations in Parkinson's disease 138

$\begin{array}{ll}\text { 9.6. Depression in Parkinson's disease } & 138\end{array}$

9.7. Subtypes of Parkinson's disease 139

9.8. Young Onset Parkinson's Disease - Clinical features, natural history and 140 mortality 
9.9. Quality of life in Parkinson's disease $\quad 140$

9.9.1. Comparison of different quality of life measures in patients with 141 Parkinson's disease

9.9.2. The impact of Parkinson's disease on quality of life 142

9.9.3. Factors that contribute to quality of life in patients with Parkinson's 142 disease

$\begin{array}{ll}\text { References } & 142\end{array}$

$\begin{array}{ll}\text { Tables } & 161\end{array}$

Figures 203

$\begin{array}{ll}\text { Appendices } & 215\end{array}$

Appendix 1. Definition of terms used in the analysis of accuracy of 215 diagnosis

Appendix 2. Quality of life instruments 216

Appendix 2.1. EQ 5D instrument 216

Appendix 2.2. Medical Outcomes Study Short Form (SF 36) 219

Appendix 2.3. PDQ 39 instrument 223

Appendix 3. Beck Depression Inventory 225

Appendix 4. Numbers of patients screened and examined in the overall 228 study 


\section{List of Tables}

Table 1. Study population by age groups and sex

Table 2. Diagnostic criteria for Parkinson's disease

Table 3. Diagnostic criteria for progressive supranuclear palsy

Table 4. Diagnostic criteria for multiple system atrophy

Table 5. Diagnostic criteria for corticobasal degeneration

Table 6. Clinical features of patients with probable or possible multiple system atrophy and progressive supranuclear palsy, and of patients with atypical parkinsonism

Table 7. Prevalence rates per 100,000 for progressive supranuclear palsy and multiple system atrophy

Table 8. Patient characteristics of all patients with parkinsonism

Table 9. Age- and sex-specific prevalence rates for Parkinson's disease and parkinsonism

Table 10. Age-specific prevalence rates for atypical, vascular, drug-induced and post dementia parkinsonism

Table 11. Breakdown of parkinsonism in different subtypes

Table 12. Initial and final diagnoses of patients seen in this population-based study

Table 13. Patients with an initial diagnosis of Parkinson's disease

Table 14. Patients with an final diagnosis of Parkinson's disease, including patients with atypical parkinsonism

Table 15. Median disease duration by Hoehn and Yahr stage

Table 16. Prediction of rate of progression

Table 17. Characteristics of patients on levodopa

Table 18. Percent of patients with motor fluctuations and dyskinesias by disease duration

Table 19. Differences between patients with and without motor fluctuations and dyskinesias

Table 20. Factors associated with significantly increased depression scores

Table 21. Comparison of the depressed and non-depressed patients on measures of impairment, disability and handicap 
Table 22. Results of stepwise multiple regression analysis of BDI scores

Table 23. Comparison of early vs late onset Parkinson's disease

Table 24. Comparison of akinetic-rigid vs tremor-dominant type

Table 25. Comparison of patients with slow vs those with fast progression

Table 26. Results of Cluster analysis to define subtypes of Parkinson's disease

Table 27. Characteristics of patients with Young Onset Parkinson's disease (YOPD)

Table 28. Clinical differences at disease onset between YOPD patients who later became demented and those who had not become demented

Table 29. Clinical differences at latest assessment demented and nondemented YOPD-patients

Table 30a. Classification of demented patients by female sex and positive family history of parkinsonism

Table 30b. Classification of demented patients by female sex, positive family history of parkinsonism, and psychiatric side effects of treatment

Table 31. Correct classification of dementia in YOPD

Table 32. Spearman correlations coefficients of different quality of life measures with clinical scales and disease duration

Table 33. Patient characteristics of respondents to Quality of life survey

Table 34. Spearman correlation coefficients of the EQ-5D summary score, subscores and visual analogue scale with the generic (SF 36)

Table 35. Clinical features associated with significantly impaired QoL scores

Table 36. The percentage of respondents reporting problems on the various dimensions of the EQ-5D by age group

Table 37. Mean scores and standard deviations of the three quality of life measures as a function of Hoehn and Yahr stage of illness

Table 38. Mean SF 36 scores, by age in comparison to the general UK population

Table 39. Mean quality of life scores in patients with Parkinson's disease in this study and previous studies

Table 40. Results of stepwise multiple regression analysis of PDQ 39 scores 


\section{List of figures}

Fig. 1 Age-specific prevalence rates of Parkinson's disease per 100,000 (95\% confidence intervals) for all United Kingdom studies of Parkinson's disease

Fig. 2 Age-specific prevalence rates of Parkinson's disease in this study and previous studies

Fig. 3. Percent of patients in each Hoehn and Yahr stage

Fig. 4. Percentage of depressed patients by Hoehn and Yahr stage

Fig. 5. Rate of prediction of depression scores by parameters of impairment, disability and handicap

Fig. 6. Number of patients with onset of Parkinson's disease before age 40, by age at onset

Fig. 7. Cumulative percentage of patients with onset of Parkinson's disease before age 40 and dyskinesias and motor fluctuations, by treatment duration

Fig. 8. Percent of patients indicating a problem on the EQ-5D

Fig. 9. Mean VAS scores of patients with Parkinson's disease compared to norms from the general population

Fig. 10. Percent of patients indicating a problem on the EQ-5D by Hoehn and Yahr stage

Fig. 11. Mean SF 36 scores, by disease severity

Fig. 12. Mean PDQ-39 scores, by disease severity 


\section{List of abbreviations}

PD Parkinson's disease

PSP Progressive supranuclear palsy

MSA Multiple system atrophy

CBD Corticobasal degeneration

YOPD Young onset Parkinson's disease

OOPD Old onset Parkinson's disease

JP Juvenile Parkinsonism

NHNN National Hospital for Neurology and Neurosurgery

CI Confidence Interval

SMR Standardised Mortality Ratio

UPDRS Unified Parkinson's Disease Rating Scale

BDI Beck Depression Inventory

MMSE Mini mental state examination

QOL Quality of life

PDQ 39 Parkinson's disease specific quality of life instrument

SF 36 Medical Outcome Studies Short Form

NPQ Niall Patrick Quinn

DIP Drug-induced parkisonism

LBPD Lewy body Parkinson's disease 


\section{Acknowledgements}

I am indebted to Professor Niall Quinn who supported me through all stages of this study with valuable advice and support, who was always available for discussions, and who was a constant and excellent teacher.

I would also like to thank Dr. Yoav Ben-Shlomo whose advice on the methodology, analysis and interpretation of epidemiological issues was invaluable.

My thanks also go to Dr. Marjan Jahanshahi for her advice on neuropsychology and statistical analysis, and Caroline Selai for her advice on measurements of quality of life.

I also thank all the general practitioners who allowed us to study their patients and who contacted them for the purpose of this study, Professor Ley Sander and Professor Simon Shorvon who allowed us to participate in the linkage scheme between the National Hospital for Neurology and Neurosurgery and several surrounding general practices, and the patients themselves.

The financial support of SmithKline Beecham Pharmaceuticals is gratefully acknowledged. 
'The government are very keen on amassing statistics - they collect them, add them, raise them to the $n$th power, take the cube root and prepare wonderful diagrams. But what you must never forget is that every one of those figures comes in the first instance from the ... village watchman, who puts down what he damn pleases.'

Sir Josiah Stamp (1929), British Statistician 


\section{Chapter 1. Introduction}

The aim of this thesis was to investigate the descriptive epidemiology of parkinsonian subtypes, with a particular view of differentiating idiopathic Parkinson's disease and atypical, neurodegenerative parkinsonian disorders. In this introductory chapter I will therefore outline the differences between these disorders and the difficulties in their differentiation, and discuss the importance of research in neuroepidemiology and the problems associated with it.

\section{$\underline{1.1 \text { Parkinsonian disorders }}$}

The clinical syndrome of parkinsonism embraces a variety of disorders, which share the three classical clinical features of bradykinesia, expressed as slowness and paucity of movement, rigidity, and, less frequently, tremor. These disorders can be classified into two major groups: Parkinson's disease and other parkinsonian disorders. Although pathologically they all share a defect in the nigrostriatal dopaminergic system, they are otherwise distinct entities with characteristic findings at post mortem examination of the brain. Despite this pathological distinction, their clinical differentiation in life is frequently difficult. In the absence of a biological marker, the diagnosis primarily rests on clinical observation, and specific features are often lacking. Late in the course of disease atypical parkinsonian disorders are often associated with additional features, but, particularly early in the course of disease, many patients are misdiagnosed. Their differentiation is however important in order to provide the patient and family with information on prognosis and possible complications, to choose the right treatment, and to not include patients in treatment trials not designed for their disease. Thus, the major diagnostic challenge for neurologists is to differentiate Parkinson's disease from this heterogeneous group of other parkinsonian disorders. 
Parkinson's disease is the most common type of parkinsonism. The main pathological correlate of Parkinson's disease is degeneration of dopaminergic pigmented neurons in the substantia nigra, and the presence of typical eosinophilic cytoplasmatic inclusion bodies, Lewy bodies, in brain stem nuclei. Parkinson's disease was first described in 1817 by James Parkinson (Parkinson, 1817), although earlier descriptions in the Indian and Chinese medicine exist (Manyam, 1990; Gourie-Devi, 1991). The classical features of bradykinesia, rigidity and tremor gradually progress, and axial features, such as bent posture, shuffling gait, postural instability, hypersalivation, speech impairment and increasing disability occur as the disease advances. Diagnosis of Parkinson's disease was thought to be straightforward, but clinicopathological studies have shown a high rate of misdiagnosis in patients dying with this diagnosis (Rajput, 1991; Hughes, 1992; Litvan, 1998).

In the last decades a number of different disorders have been recognized that can be associated with clinical features similar to those of Parkinson's disease. Most importantly, other and rarer neurodegenerative diseases such as multiple system atrophy, progressive supranuclear palsy, and corticobasal degeneration, which are pathologically characterized by entirely different correlates, can mimic the clinical picture of Parkinson's disease. The pathological abnormalities in multiple system atrophy extend beyond the substantia nigra, involving the striatum (mainly putamen), the inferior olives, pons and cerebellum, and the intermediolateral cell columns and Onuf's nucleus in the spinal cord. Oligodendroglial cytoplasmatic inclusion bodies (GCIs) are characteristically present, widespread and plentiful, and Lewy bodies absent, unless incidental (Lantos, 1998; Wenning, 1995). In brains of patients with progressive supranuclear palsy, neuronal loss and gliosis with neurofibrillary tangles and neuropil threads in high density are found in a characteristic distribution in the basal ganglia and brainstem (Hauw, 1994). In corticobasal degeneration asymmetrical neuronal loss and gliosis with swollen 
neurons is mainly found in the parietal and frontal cortex with variable involvement of subcortical structures (Gibb, 1990). Due to the more extensive neuronal loss in these disorders, clinical features indicating the more widespread involvement of other structures within the central nervous system typically occur, and often do not respond well to treatment with levodopa or other antiparkinsonian drugs. Thus, multiple system atrophy can cause cerebellar features, pyramidal signs, and early autonomic features, which do not occur in Parkinson's disease, and other features such as prominent antecollis, discoloured cold hands, involuntary sighs, stridor, newly developed snoring or permanent restriction to a wheelchair may also be present (Wenning, 1994a). Progressive supranuclear palsy, on the other hand, may present with early falls, often as the initial feature, often with little and frequently symmetrical limb akinesia. When a vertical supranuclear gaze palsy develops, usually later in the disease, a diagnosis can be made, but in some patients it may not develop at all (Litvan, 1996a). Corticobasal degeneration is even rarer than multiple system atrophy or progressive supranuclear palsy, and is often characterized by the pathognomonic 'alien limb', which patients described as having 'a mind of its own'. Classically, cortical sensory loss, apraxia and myoclonus in addition to parkinsonism occur, but can be absent in some patients (Gibb, 1989a; Litvan, 1997b). However, these fully developed classical syndromes often do not develop until late, and their distinction from Parkinson's disease poses a particular challenge to the neurologist. Not only can these disorders mimic Parkinson's disease, but may also be indistinguishable from each other (Litvan, 1997b). Expert clinical examination, application of strict diagnostic criteria, long-term follow-up, and eventually pathological examination of the brain would therefore be desirable for correct diagnosis. In clinical practice, however, this can rarely be achieved, and many of patients remain undiagnosed until death (Hughes, 1992). 
In addition to these atypical neurodegenerative diseases, parkinsonism can also be caused by other diseases, or as a consequence of drugs or toxins. A variety of disorders affecting the function of the basal ganglia, such as basal ganglia lesions, intoxication with carbon-monoxide or manganese, metabolic disorders such as Wilson's disease, metachromatic leukodystrophy, or neuroacanthocytosis, and other, neurodegenerative syndromes like Huntington's disease, dementia with Lewy bodies (which may be part of the same disease spectrum as Parkinson's disease), or Alzheimer's disease can cause secondary parkinsonism. However, in these disorders, other features are usually present, and confusion with Parkinson's disease is less frequent. Longterm treatment with dopamine depleting drugs such as neuroleptic drugs or antiemetics, however, can induce a, usually reversible, parkinsonian syndrome, which can otherwise be indistinguishable from idiopathic Parkinson's disease. Moreover, in many such cases underlying Parkinson's disease may only have been unmasked by the use of the dopamine antagonist (Bower, 1999).

To complicate matters further, some symptoms and signs of parkinsonism can be confused with other non-parkinsonian disorders. Vascular disease or hydrocephalus can cause a small stepped gait and postural instability, and benign essential tremor or other forms of tremor can be mistaken for parkinsonian tremor. Conversely, patients with early, particularly benign tremulous Parkinson's disease or elderly patients with Parkinson's disease are sometimes not diagnosed with the disorder, as their symptoms may be misdiagnosed as benign or 'senile' tremor or attributed to old age. Often, these other disorders can be differentiated by careful history taking and examination, but many of these patients may, nevertheless, only be correctly diagnosed at post-mortem examination.

As a consequence, in clinico-pathological studies up to $35 \%$ of all patients who die with a diagnosis of Parkinson's disease have a different 
diagnosis at post-mortem pathological examination (Rajput, 1991; Hughes, 1992). The main differential diagnoses in these series were progressive supranuclear palsy, multiple system atrophy, Alzheimer's disease, vascular disease, and essential tremor. This rate of incorrect diagnosis was shown to be improved by application of rigid clinical criteria that were derived from such clinico-pathological studies (Hughes, 1992), although a minority of patients never develop typical features of their disease even until death.

\subsection{Epidemiology}

Epidemiology is concerned with the study of distribution and dynamics of diseases in human populations (Schoenberg, 1977). The term epidemiology is derived from the Greek terms epi (upon), demos (people) and logos (doctrine or science), and was originally used to describe outbreak of infectious disease in large population groups (Schoenberg, 1977). In modern times, the use of the term epidemiology has widened. The main roles of epidemiological research today are the estimation of the magnitude of disease burden in the population, the provision of aetiological clues, accurate description of the natural history of disease, and the measurement of the impact of the disease and interventions. Thus, epidemiological studies are an important tool to plan the provision of health care resources, to investigate patterns of occurrence and risk factors, to predict prognosis, to determine the needs of patients with the disease, and to ultimately determine the cause of disease and plan its prevention. For these reasons, epidemiology has been increasingly recognized as an area of major importance by the World Health Organization, and its prime importance has been highlighted by the NHS Research and Development executive (Beecham, 1992), and by the newly appointed director of NHS Research and Development, Professor Michael Peckham in his inaugural lecture. 
Descriptive epidemiology characterizes observations of disease in human populations by attempting to identify and study all cases in a defined population. Two of the key measurements used in descriptive epidemiology are the prevalence and incidence of a disease (Schoenberg, 1981). Prevalence measures the number of all current cases of disease within a specific population at a given time and place. The incidence is the number of new cases of a disease occurring in a defined population and period of time, usually over a year's time. It can be calculated indirectly by dividing prevalence by the average duration of the disease (Schoenberg, 1981). Changes over time or differences between geographical areas can serve to identify patterns of disease occurrence. The determination of prevalence or incidence figures for a particular disease requires the ascertainment of all cases of the disease in the target population and high diagnostic accuracy. Major sources of error in prevalence and incidence studies are therefore underascertainment, caused by incomplete detection of cases in the study population, and overascertainment, caused by inclusion of false positive diagnoses.

Descriptive epidemiology is also concerned with the description of clinical features, natural history, and prognosis of disease, thus providing a fuller picture for our understanding of the disease. Examples are the description of the rate of progression, occurrence of remission and mortality of the disease, identification of subgroups, factors determining prognosis or general impact, and identification of needs in the population affected by the disease (Schoenberg, 1977).

The other main area of epidemiology, analytic epidemiology, is mainly concerned with the cause and control of disease (Schoenberg, 1982). It aims at identifying factors that are associated with a low or high risk of disease by comparing those with the disease to a control group, either in a prospective study or retrospectively in a case control study. Experimental epidemiology 
similarly aims to identify risk factors for disease, but this time by exposing individuals with the disease prospectively to experimental factors in a controlled fashion, for instance in a randomized trial.

Any kind of epidemiological research however has to fulfil two crucial requirements: 1) representativeness and 2) accuracy of diagnosis. The individuals studied (study population) must have the same or similar characteristics as the population under investigation (target population). In order to be able to generalize the results to other areas, this target population should also be similar to the overall population. Thus, a study in inhabitants of a retirement community might yield very different results from a study in a small University town where a third of the population are students, and the exclusive examination of individuals attending a sports centre will hardly be representative of the health status of the population.

In order to obtain valid results from epidemiological research it is also crucial not only to choose a population representative of the population one wishes to study, with ideally inclusion of all suitable subjects within this population, but also to exclude all subjects who do not have the disease under investigation. Contamination of patient samples with cases of different diagnoses can alter the results greatly, as the frequency, distribution, causes, risk factors and prognosis may be very different between different diseases. For instance, inclusion of patients with essential tremor among a population of patients with Parkinson's disease will yield higher prevalence rates, slower progression, better prognosis, and increase the rate of inheritance. Establishing the correct diagnosis is therefore a pivotal requirement of epidemiological research.

Epidemiological studies of neurological disorders have been burdened with major obstacles: Clinic-based studies are not representative for the population of all patients with the disease due to the substantial referral bias that is inherent to such samples. Population-based studies are therefore 
required to study a population that is representative of the whole population of patients with this disorder. On the other hand, in many neurological diseases, such as parkinsonism, no biological marker is available and diagnosis relies on clinical examination. Thus, expert assessment of clinical findings is essential to obtain high diagnostic accuracy. However, many neurological diseases are rare and, to minimize the likelihood of chance findings, epidemiological studies require large populations. A door-to-door survey, which aims to screen all individuals in the target population, is the method with the greatest sensitivity to detect all cases of disease in the population. However, examination of all individuals is impractical, especially if a high level of diagnostic accuracy with expert examination is attempted. They are labour-intensive and costly projects, even if performed with the help of trained helpers who are medically unsophisticated. Additionally, even in studies of this kind, some individuals with the disease may be missed, as a) not everyone may agree to participate, b) the screening criteria may not pick up 100 percent of cases, and c) many neurological diseases like Parkinson's disease start gradually with little or no findings in the initial stages. Among the alternatives, the next most complete method of case ascertainment is to screen the target population at primary care level, which requires that an individual has sought medical advice for a given symptom, or a finding was detected by a medical person incidentally. Although individuals who have not yet come to medical attention will not be captured with this method it does not require referral to a hospital or specialist, prescription of specific medication or even diagnosis of the disorder, as the symptoms are in themselves sufficient for inclusion in the study. However, this method is often not possible as a) the patient populations of primary care physicians in many countries are not necessarily representative of the study area, which introduces a selection-bias, b) primary care physicians are not necessarily aware of their patients attending other doctors or hospitals, and c) the practices may not have a computerized 
system from which information is easily retrievable, thus relying on the physicians' memory. If, as in the United Kingdom, the health care system is organized locally, with the general practitioner as the 'gatekeeper' to specialist medical care, these problems are avoided and underascertainment minimized. The third way of case ascertainment is using multiple sources, such as hospital records, nursing home records and drug-prescriptions. Identification of all suitable subjects then usually relies on medical records from a variety of different sources. The risk of incomplete case ascertainment is greater with this method than with the former two, as patients early in the disease may not be included due to the difficulties in early recognition of a slowly progressive disorder such as parkinsonism, and some patients may not be picked up by any of the sources. The least representative method of case ascertainment is that of obtaining data on a population from just one of these sources, such as from a hospital covering the area, or from records on patients receiving specific drug-prescriptions. This method may lead to overascertainment, e.g. through patients being referred from a different catchment area or receiving these drugs for a different disease, or underascertainment, e.g. by excluding patients who are not referred to this hospital or do not require or tolerate these drugs.

The validity of the results of epidemiological research relies not only on completeness of case ascertainment and representativeness, but also on the correct diagnosis. Too wide inclusion criteria, such as inclusion of all patients on antiparkinsonian drugs or all patients with tremor or a diagnosis of parkinsonism among patients with Parkinson's disease, will result in artificially high prevalence rates and can result in distortion of the conclusions on prognosis and aetiology of the disease, and ultimately inhibit or delay the finding of the cause and possible prevention. Too rigid diagnostic criteria, such as inclusion of only patients who present with the typical clinical syndrome, on the other hand, will result in the exclusion of true cases of the 
disease, which may not have fully developed, thus underestimating the extent of the disease and distorting the results in the opposite direction. The clinical diagnosis and the application of defined clinical criteria are therefore the key variables in the provision of epidemiological data.

\subsection{Descriptive epidemiology of parkinsonism}

In the case of parkinsonian disorders, the task of diagnostic accuracy is conspicuously difficult, and highly important at the same time. The difficulties and pitfalls in the diagnosis of parkinsonism can lead to over- or also underdiagnosis of Parkinson's disease. Epidemiological data on the prevalence of Parkinson's disease are reliant on correct diagnosis, and the described difficulties in diagnosing Parkinson's disease limit the determination of its true extent. Many studies on the prevalence of Parkinson's disease not only employed different methodology and case ascertainment methods, but also differing inclusion and exclusion criteria, sometimes resulting in the inclusion of a variety of other disorders, e.g. vascular parkinsonism, and drug-induced parkinsonism. The failure to differentiate Parkinson's disease from other parkinsonian syndromes, the varying use of diagnostic criteria, the subtle onset of the disease, the possible overlap with symptoms of aging, such as postural instability and slowness all contribute to the varying results of prevalence studies of parkinsonism. These diagnostic difficulties are therefore partly responsible for the very differing results of epidemiological studies of Parkinson's disease, resulting in prevalence rates ranging from 57 to 234 cases of Parkinson's disease per 100,000 people (Zhang, 1993), and in sometimes conflicting conclusions on the subtypes, characteristics or prognosis of Parkinson's disease (Jankovic, 1990; Graham, 1999; Hershey, 1991; Kostic, 1991). In addition, conclusions on the aetiology, natural history and impact of individual parkinsonian syndromes rely importantly on the differentiation of true cases of parkinsonism, uncontaminated by different disorders and known 
causes of parkinsonism. Contamination of samples of patients with Parkinson's disease may thus not only result in an overestimation of the rate of Parkinson's disease in the population, it may also lead to the distortion of the results of clinical studies on risk factors, complications and prognosis of Parkinson's disease. Atypical parkinsonian disorders can not only be associated with a number of additional features, but also progress faster towards greater disability and earlier death than patients with Parkinson's disease (Poewe, 1998; Wenning, 1994a). Thus, the faster rate of progression and the higher rate of mortality of atypical neurodegenerative parkinsonism would introduce a bias to a much worse prognosis of Parkinson's disease, and the lack of response to treatment may result in more severely impaired quality of life than in a sample of patients with typical Parkinson's disease only. The rate of progression in early studies of Parkinson's disease, which probably included patients with postencephalitic parkinsonism as well as those with atypical parkinsonian disorders, some of which were not recognised at the time, thus resulted in greater variability of rates of progression and greater mortality (Hoehn and Yahr, 1967) than later studies that excluded these patients (Martilla, 1977; Ben-Shlomo, 1995). In addition, disability may result from very different complications of the disease in atypical parkinsonian disorders than in Parkinson's disease, such as falls, speech and swallowing difficulties, or cognitive impairment, whereas other problems, such as motor fluctuations and dyskinesias may be more likely to compromise quality of life in patients with Parkinson's disease.

In recent years, it has become possible to directly investigate quality of life or subjectively evaluated health status, with validated and reliable instruments in patients with Parkinson's disease (Fitzpatrick, 1997; Rubenstein, 1998). It is increasingly recognised as one of the most important evaluation and outcome measures in management of these patients (Devinsky, 1995). However, unless rigorous exclusion criteria are applied, patients with 
atypical parkinsonian disorders will inevitably be included and studies will thus result in lower quality of life than in a sample exclusively including patients with Parkinson's disease (Fitzpatrick, 1997; Peto, 1995). It is thus crucial for the interpretation of all epidemiological and clinical studies that a high level of diagnostic accuracy is achieved and cases with atypical disorders are excluded.

These methodological and diagnostic difficulties not only have consequences for studies on the epidemiology of Parkinson's disease, but also of atypical parkinsonian disorders. These disorders, such as multiple system atrophy and progressive supranuclear palsy, carry a more severe prognosis than Parkinson's disease and have different, but still unknown, aetiologies. However, very little is known on their prevalence in the population. As patients with these disorders are often difficult to distinguish from patients with idiopathic Parkinson's disease, they are frequently not correctly diagnosed and misdiagnosed as Parkinson's disease. They are comparatively rare and have only been described in the last decades (Graham and Oppenheimer, 1969; Steele, 1964; Rebeiz, 1968). As a consequence, they are often not recognized in population-based epidemiological studies, which were not designed to detect these disorders. These studies usually employ methods of case ascertainment and neurological evaluation with a low sensitivity for the recognition of atypical parkinsonism, and either did not distinguish them from Parkinson's disease, or excluded them from the study population (Tandberg, 1995; Morgante, 1992). The results of studies based on pathological examination of brains of patients with parkinsonism suggest that these disorders are less rare than clinically suspected, and represent a substantial proportion of patients dying with a diagnosis of Parkinson's disease (Hughes, 1992). However, studies derived from brain banks, and also from specialist clinics, are likely to overrepresent these disorders 
(Maraganore, 1998), and it is unknown how frequently these disorders occur in the unselected population.

In this thesis I will report on a descriptive epidemiological study of the different types of parkinsonism, which was undertaken with a particular emphasis on diagnostic accuracy of the parkinsonian disorders. Five main areas were investigated. First, the prevalence and incidence of parkinsonism and its different subtypes, and their relative distribution among patients with parkinsonism. Second, the accuracy of diagnosis of these disorders in the community. Third, the description of the clinical syndrome of Parkinson's disease (the most common of these subtypes and the only one to allow meaningful further analysis) with its main complications and the factors contributing to them in this population, and the analysis of different subtypes of Parkinson's disease. Fourth, the analysis of quality of life in this population-based sample of patients with Parkinson's disease, and the factors determining it. 


\section{Chapter 2. Aims of the study}

\subsection{The prevalence and incidence of parkinsonian disorders in the population}

1. To determine the population prevalence and incidence of atypical parkinsonian disorders, such as multiple system atrophy and progressive supranuclear palsy

2. To determine the prevalence and incidence rates of Parkinson's disease in the area of London and to compare these with previously found prevalence and incidence rates in other areas

3. To calculate the distribution of parkinsonian disorders among patients with parkinsonism in this population

\subsection{Accuracy of diagnosis}

1. To determine the rate of true and false positive cases of patients diagnosed with Parkinson's disease and related disorders in the community

2. To determine the rate of true and false negative cases of Parkinson's disease and related disorders among all patients who had already come to medical attention in the community

\subsection{The clinical features and natural history of parkinsonian disorders in a population-based sample}

1. To analyse the frequency of symptoms and signs of the disease in patients with parkinsonian disorders in a cross-sectional sample of patients with these disorders

2. To determine the factors influencing disability in this cross-sectional sample of patients with Parkinson's disease

3. To retrospectively investigate the rate of progression and factors associated with fast progression in this cross-sectional sample of patients with Parkinson's disease

4. To investigate the rate of motor complications and the factors determining their occurrence in patients treated with levodopa

5. To investigate the rate of depression in a population-based sample of patients with Parkinson's disease and the factors that influence their occurrence

6. To determine the different subtypes of Parkinson's disesase 
7. To analyse the features and prognosis of Parkinson's disease in patients in whom it starts before the age of 40 years

\subsection{Quality of life in Parkinson's disease}

1. To investigate quality of life in a population-based sample of patients with Parkinson's disease using a disease-specific and two generic quality of life instruments.

2. To compare the validity and reliability of these three different quality of life questionnaires in patients with Parkinson's disease

3. To compare impairment of quality of life in patients with Parkinson's disease to that of people of the same age group and sex in the population 


\section{Chapter 3. Methodology}

The primary aim of this study was to obtain prevalence rates and clinical data on a representative sample of patients with the different types of parkinsonism classified with high diagnostic accuracy. In order to achieve this aim, rigid diagnostic criteria, which were mainly derived from clinico-pathological studies, were employed, and as many patients as feasible with possible parkinsonism in a representative target population were included in the screening phase of this study. A population-based approach was used, employing a linkage scheme with a number of general practices, who used computerised patient records, to identify all patients with possible parkinsonism in a population of over 100,000 people in the area of London.

\subsection{Patient ascertainment}

The most sensitive method of patient ascertainent, door-to-door surveys, require large human and financial resources, and therefore the next sensitive method, a populationbased survey with patient ascertainment via general practices was chosen. Primary care in the UK is organised locally and coverage of the population is high, with more than $98 \%$ of residents being registered with a general practitioner (Cockerell, 1993). The study population was therefore considered representative of the area of investigation.

\subsubsection{Linkage Scheme with general practices}

A linkage scheme between the National Hospital for Neurology and Neurosurgery (NHNN) and a number of general practices in and around London has been established since 1994 (Cockerell, 1996). Patients with neurological syndromes were referred from these general practices to the NHNN in an incidence study of neurological disease, which has been conducted for more than five years. One practice, which was also the biggest practice, was situated in Tonbridge, Kent (Warders Medical Centre), and the remaining practices in London. The patients belonged to different Health Authorities within London, including Fulham (Cassidy Medical Centre), Westminster and Pimlico (Westminster and Pimlico General Practice), and Hackney (The Lawson Practice), but the majority belonged to the Camden and Islington Health Authority (The Amwell Street Practice, Lonsdale Medical Centre; The Surgery, Mitchison Rd; The Holborn 
Medical Centre, The Museum Practie, Abbey Medical Centre, River Place Health Centre; The St. John's Way Medical Centre, The Caversham Group Practice, James Wigg Practice; Elizabeth Avenue Group Practice). The practices were included if they a) had computerised records with diagnoses and treatments to choose from a stardardised menu to enable easy and complete retrieval of information on diagnoses and treatment, b) were located in an area considered typical for the overall population, e.g. not primarily responsible for a nursing home population, c) were not involved in other population-based studies on diagnosis or treatment of Parkinson's disease in order not to confound the results.

All of the general practices in the linkage scheme were invited to participate in this study on the epidemiology of parkinsonian syndromes. All of them agreed to participate, but two practices (Bounds Green Group Practice and The Surgery, Fitzroy Square) had been excluded from the trial as both were already participating in a different study involving patients with Parkinson's disease. It was felt that a second study would be confusing and too great a burden for these parkinsonian patients, and that it could also confound the results of this and the other study, which examines the effects of nursing intervention in parkinsonian patients in a controlled study. The remaining general practices had an overall population of 89,821 . To cover a population of more than 100,000 persons a number of other general practices were contacted and invited to participate in the study. Only surgeries with three or more partners were contacted in order to exclude practices who did not use computerised records, and to achieve the highest possible consistency of screening methods. Three additional general practices belonging to the Camden and Islington Health Authority, covering a population of 31,787 on prevalence day, agreed to participate. The population figures by age and sex of all general practice on prevalence day is given in table 1 .

All general practices agreed to participate in this study after discussing its purpose, method and the potential benefit and burden for the patients and the practice among the partners. One practice (The Amwell Street Practice), whose partners had only recently taken over the practice, requested that the patients were contacted by me rather than by themselves. 


\subsubsection{Patient searches}

\subsubsection{Stage I}

The computerised records of all general practices were screened according to the same procedure and criteria, where possible. The search to detect patients with possible parkinsonism was performed on all patients who were registered with the practice on

prevalence day, the $1^{\text {st }}$ July 1997, and entries from the earliest entry to the day of search were included. A threefold search was performed using the following criteria:

1. All patients with a current or previous diagnosis of parkinsonism, without restriction of age, including paralysis agitans, parkinsonism secondary to drugs, malignant neuroleptic syndrome, postencephalitic parkinsonism, secondary parkinsonism due to other external agents, secondary parkinsonism unspecified, and Parkinson's disease not otherwise specified.

2. All patients with a current or previous diagnosis of tremor or those in whom a tremor had ever been recorded. No type of tremor and no patient with a tremor was excluded at this stage. Included were codings for 'has a tremor', 'trembling', 'fine tremor', 'intention tremor', 'coarse tremor', 'parkinsonian tremor', 'familial tremor', 'essential and other specified tremor', 'drug-induced tremor', 'other specified forms of tremor', and 'tremor not otherwise specified'.

3. All patients who had ever received a drug used in parkinsonism or related disorders, including dopaminergic and anticholinergic drugs used in parkinsonism, 1-dopa, drugs used in essential tremor, chorea, tics and related disorders, Britaject, apomorphine hydrochloride, Tasmar and tolcapone (after they were made available). The only drugs excluded from this search were botulinum A toxin, Botox, and Dysport injections, Nitoman and tetrabenazine, as they are not used for the treatment of parkinsonism, but of other movement disorders.

4. All patients who were diagnosed or suspected to have 'progressive supranuclear palsy' or 'progressive supranuclear ophthalmoplegia'.

5. All patients who were diagnosed or suspected to have 'striatonigral degeneration', 'Shy-Drager syndrome', or 'parkinsonism with orthostatic hypotension'.

6. All patients with 'other basal ganglia disorders not otherwise specified' were screened for symptoms suggesting any of the above. 
The same search was repeated in all practices at least once during, and at the end of the study to detect newly diagnosed patients, or patients who had developed parkinsonism or tremor during the time of the study.

\subsubsection{Stage II}

The personal records of all patients identified in stage I, on computer and written notes, were examined to determine whether the patients were correctly coded and met the inclusion and exclusion criteria (see below), and to extract as much clinical information as possible.

For each practice, a list of all patients who fulfilled the inclusion criteria and did not fulfil any of the exclusion criteria was constructed, including patients in whom insufficient information was available to determine whether the inclusion or exclusion criteria applied. For instance, in a number of, particularly elderly, patients, the onset of tremor was not recorded, but it was present at the time when the patient joined the practice after the age of 50 years. Or alternatively, tremor was mentioned in the notes as a known problem, but the onset was not recorded. These patients were also included on the preliminary list of eligible patients.

\subsection{Inclusion criteria}

I. All patients in whom a diagnosis of parkinsonism was made or the suspicion of it was recorded at any point in time, and

II. All patients with progressive supranuclear palsy, progressive supranuclear ophthalmoplegia, striatonigral degeneration, Shy-Drager syndrome, parkinsonism with orthostatic hypotension, and other basal ganglia disorders not otherwise specified with symptoms suggesting any of the above, and

III. All patients with a tremor in the past or at present, and

IV. All patients who had ever received antiparkinsonian drugs.

\subsection{Exclusion criteria}

Regarding I. Patients with parkinsonism 
Drug-induced parkinsonism (treatment with dopamine receptor blocking agents at and at least 6 months prior to the onset of symtoms)

Parkinsonism following dementia (onset of dementia clearly before the onset of symptoms of parkinsonism)

Regarding III. Patients with tremor

Tremor starting before the age of 50 years

Drug-induced tremor (treatment with dopamine receptor blocking agents at, and at least 6 months prior, to the onset of tremor, or tremor in clear relationship to treatment with lithium)

Tremor due to thyrotoxicosis

Tremor due to alcohol withdrawal

Regarding IV. Patients on antiparkinsonian drugs

Patients on antiparkinsonian drugs for a different indication (e.g.

bromocriptine for prolactinoma, procyclidine in combination with a neuroleptic drug for treatment of schizophrenia)

Miscoded patients (e.g. spouse of a patient who was mistakenly listed to receive antiparkinsonian treatment)

All patients who were eligible for the study according to the defined criteria and registered with the practices on prevalence day, were included. Patients who had screened positive at the beginning of the study, but were not part of the practice population on prevalence day, as they had moved or died, were excluded from the study, independently of whether they had been seen or not. Patients who were diagnosed with a tremor or parkinsonism, or who received an antiparkinsonian drug after prevalence day, were also excluded from the study.

\subsubsection{Stage III}

The general practioners decided whether the patients were listed correctly and were eligible for the study. Few patients were excluded by their general practioners because he or she had felt that the patient was in too poor a condition to participate in the survey. These patients were included in the group of patients who had declined to participate. 


\subsubsection{Patient contact}

A letter inviting each patient to participate in the study on headed paper from the general practice was signed by the patient's general practitioner and returned to the researchers. This letter was sent to each patient along with the patient information sheet, a stamped addressed envelope, and a return form to indicate refusal or agreement to participate. All patients were given the option to be seen at the NHNN, at their general practice or at their home. In any contact with the patients the study was named 'Study on slowness and tremor' to avoid mentioning the word 'Parkinson's disease', in order not to alarm the patient.

In patients who declined to participate, as much information as possible was extracted from the computerised and written notes in the general practices. Additionally, the records of the NHNN were screened to determine whether the patients had been seen there before, and if so, information was extracted from the notes.

Patients who did not reply received a reminder letter after 4 weeks, and a second after another 2 weeks if they failed to answer. If there was still no reply, the general practitioner was contacted to find out whether the patient was still alive and residing in the area, and if so, he or she received a reminder call.

\subsection{Patient assessment}

\subsubsection{Diagnostic visit}

If patients agreed to participate, they were contacted by telephone or in writing and an appointment was made at the NHNN, at the general practice, or at their home. All patients were seen by me, and patients who were seen at the NHNN were mostly seen together with Professor Niall Quinn. In addition to clinical history taking and a complete neurological examination, a questionnaire with ticklist for the presence or absence of symptoms and signs, specifically designed for the purpose of this study in order to detect any typical and atypical features of parkinsonism, was completed. As a subsection of this, the UPDRS motor part (Fahn, 1987) was applied. In addition, a short cognitive test, using the Minimental scale of Folstein (Folstein, 1975) was performed. 
A Hoehn and Yahr scale (Hoehn and Yahr, 1967), as well as a Schwab and England grading (Schwab and England, 1969) was performed. Patients were also asked to rate their disability on the Schwab and England scale themselves. Patients were questioned about the presence or absence of a number of symptoms and signs associated with Parkinson's disease and atypical parkinsonian syndromes, such as hallucinations, falls (and their first occurrence), gait impairment, motor fluctuations or dyskinesias, incontinence, orthostatic symptoms (and their severity), insomnia, visual disturbances, pain, speech or swallowing impairment or a family history of Parkinson's disease. They were also asked which single symptom had been present at onset and whether they were unemployed or had retired early due to having parkinsonism. Details of the patients' medical history and medication, including time of diagnosis and initiation of treatment, were extracted from their general practitioners' records. If the dose of antiparkinsonian treatment listed in the records differed from that the patient and carer reported as actually taken, the dose reported by the patient and carer was taken as the current dose.

\subsubsection{Diagnosis. A diagnosis was made according to published criteria (see below).} If necessary, further investigations were recommended. In all patients who were seen at home, at the general practice, or at the NHNN in the absence of NPQ, a video recording of the patient was taken according to a defined protocol (sitting at rest, speaking, rapid alternating movements, holding both arms extended, whole body and zoomed to different body regions, walking, handwriting, finger-to-nose test, eye movements), and all patients were discussed with the consultant who also reviewed the video, but was blinded to the initial clinical diagnosis.

In cases in whom an unequivocal diagnosis could not be made without a remaining doubt, a repeat visit was arranged for the end of the study, either at the NHNN, the general practice or at home. At this second visit, a repeat video recording was taken and reviewed, if the patient was not seen by the consultant personally, and a final diagnosis was made, if possible. 
3.2.1.2. Diagnostic criteria. Parkinsonism was diagnosed if bradykinesia and at least one other of the four cardinal signs (resting tremor, rigidity, or impaired postural reflexes) were present. Parkinson's disease was diagnosed according to the UK PD Society Brain Bank criteria (Gibb, 1989b; table 2) with the exception that patients with an isolated Babinski sign but otherwise typical Parkinson's disease were not excluded. In addition, patients with only a classical resting tremor were included as 'possible Parkinson's disease'. Progressive supranuclear palsy (probable and possible) was diagnosed according to criteria proposed by the National Institute of Neurological Disorders and Stroke (NINDS) and the Society for Progressive Supranuclear Palsy, Inc. (SPSP; Litvan, 1996a; table 3), and multiple system atrophy (probable and possible) according to the criteria of Quinn (Quinn, 1994; table 4). The diagnosis of CBD was made according to the criteria of Litvan (Litvan, 1997a; table 5). Vascular pseudoparkinsonism was diagnosed when there was presence of at least two of the following: history of previous strokes, abrupt onset with stepwise progression, hypertension, a wide-based gait with small steps, cognitive decline, pseudobulbar or pyramidal signs (Zijlmans, 1996).

\subsubsection{Depression and Quality of Life assessment}

At the first visit, all patients with a clinical diagnosis of parkinsonism, who agreed to it, were given a questionnaire booklet comprising the Beck Depression Inventory, a widely used 21-item depression inventory (BDI; Beck, 1961), and three different quality of life questionnaires, a disease-specific instrument for patients with Parkinson's disease, the PDQ 39 (Peto, 1995), and two generic, non-specific quality of life instruments, the EQ-5D (EuroQoL Group, 1990) and the Medical Outcomes Study Short Form (SF 36; Brazier, 1992). Patients were also asked to rate their current general level of health as better, much the same, or worse than during the past 12 months. They were asked to complete this questionnaire as soon as possible, and return it to the researchers in a stamped addressed envelope, which was provided.

\subsubsection{Follow-up}

All patients with a diagnosis of parkinsonism, who agreed to this, were sent a brief questionnaire at 3-monthly intervals with questions on development of additional 
symptoms or aggravation of symptoms like depression, hallucinations, confusion, falls, fluctuations of motor performance, dyskinesia, or forgetfulness during these last three months. Secondly, the general practitioners were asked at the end of the study whether they had noticed any new atypical features in any of their eligible patients. If any atypical features or features suggestive of atypical parkinsonism were detected a second visit was arranged at the end of the study (end of 1998)

\subsection{Data analysis}

For all analyses only patients who were registered with the involved general practice on prevalence day and fulfilled inclusion criteria on prevalence day were included. Thus, patients who had been seen and diagnosed, but had died or left the area before prevalence day, or those who developed parkinsonian features after prevalence day or moved into the area after it, were not included in the analysis. The akinesia score of the UPDRS was calculated as the sum of items 19 and 23 to 26 of the UPDRS for both sides, the score of axial features from items 27 to 30 , and the tremor score from items 20 and 21 for both sides. The tremor-dominant subtype of PD was defined as patients with a ratio of tremor to bradykinesia score (bradykinesia, rigidity and postural instability subscores from the UPDRS motor scale and history) $\geq 0.5$, and the akineticrigid subtype as patients with a ratio of $<0.5$. Socioeconomic groups were determined according to the classification of occupations and coding index (Office for Population indices, 1980).

Group differences were analysed by the student's t-test. Categorical data were compared with Chi-square test, and Fisher's exact test if the numbers were small. Spearman rank correlations were calculated for interval data. A statistical level of 5\% was considered as significant in most analyses. However, in order to take into account multiple comparisons in some analyses, a statistical threshold of $\mathrm{p}<0.005$ was considered to be sufficiently conservative for these analyses. Differences with $\mathrm{p}<0.01$ and $p<0.05$ were then considered as marginally significant. The statistical analyses of data will be further described in each individual chapter.

\subsection{Approval by an Ethics Committee}


The study and its design, including the methods of patient ascertainment, contacting eligible patients, the patient information sheet, the examination procedure, and followup questionnaires and examination were approved by the Joint Medical Ethics Committee of the National Hospital for Neurology and Neurosurgery and the Institute of Neurology.

\subsection{Confidentiality and patient consent}

The initials of all patients, their date of birth and their general practitioner were recorded on hard copy and computer system. No information was passed on to any third party, apart from a summary letter on the clinical findings, diagnosis and management suggestions, where appropriate, to the general practitioner, unless the patient declined this. 


\section{Chapter 4. The population prevalence and incidence of the atypical parkinsonian disorders progressive supranuclear palsy and multiple system atrophy}

\subsection{Background}

Progressive supranuclear palsy (PSP) and multiple system atrophy (MSA) are two distinct and well described neurodegenerative disorders but little is known about their prevalence and incidence in the general population. They are frequently misdiagnosed, particularly as Parkinson's disease (PD). The diagnosis may be particularly difficult in the early stages of the disease before atypical features have clearly emerged, so that ideally patients should be regularly reassessed by movement disorder specialists (Rajput, 1991). In the United Kingdom Parkinson's Disease Society Brain Bank, neuropathological examination of patients who died with a diagnosis of PD showed that around $6 \%$ and $5 \%$ of them actually had PSP and MSA respectively (Hughes, 1992), and among a series of 35 pathologically proven cases of MSA, 55\% of the 22 cases referred from other institutions died with an erroneous diagnosis of PD (Wenning, 1995). Over the 5 year period from 1989 to $1994,7.9 \%$ and $9.9 \%$ of 303 brains donated had PSP and MSA, respectively (Dr. Susan Daniel, personal communication). However, PSP and MSA patients are likely to be over-represented in studies of PD brain banks (Maraganore, 1998), and therefore extrapolating from these results to estimate their prevalence may produce very misleading estimates. Moreover, because survival in PSP and MSA is markedly shorter than in PD, such figures are a better indicator of incidence than prevalence.

There are no large population-based studies on the prevalence of PSP and MSA. Prevalence studies of PD usually either exclude atypical parkinsonian patients or do not attempt to diagnose such patients but simply include them under the diagnosis of parkinsonism. (Tandberg, 1995; Morgante, 1992). This population-based study was primarily carried out to determine the prevalence rates of PSP and MSA.

\subsection{Methods}

The methods of case ascertainment and diagnosis were described in chapter 3 . In brief, the computerised records of 15 general practices (12 participating in the linkage 
scheme and 3 additional practices; 14 in London and 1 in Kent) were screened for patients with the following entries: 'Parkinson's disease', 'parkinsonism', 'progressive supranuclear palsy', 'striatonigral degeneration', 'Shy-Drager syndrome', 'parkinsonism with orthostatic hypotension', and 'other extrapyramidal disorders, not otherwise specified', 'tremor' with onset above the age of 50 years, as well as for those patients who had ever been prescribed an antiparkinsonian drug. This over-inclusive multiple approach was taken to ensure that the screening criteria had a high sensitivity (include all PSP and MSA cases) at the expense of low specificity (include lots of other conditions). Patients with only tremor beginning before the age of 50 were excluded because the risk of missing PSP or MSA patients presenting at a young age with isolated tremor without other neurological signs was considered minimal. I reviewed the computerised and hard copy records of all eligible patients. Patients were excluded from further evaluation in this part of the study if they (a) had used antiparkinsonian drugs for other indications (e.g. bromocriptine for pituitary adenoma or anticholinergic drugs taken 'prophylactically' in association with dopamine antagonists in patients with psychotic disorders), (b) had a known alternative cause of tremor (e.g. hyperthyroidism), (c) had onset of parkinsonian symptoms within 6 months of at least 6 months treatment with dopamine receptor blocking drugs, (d) developed dementia before the onset of their parkinsonism, and (e) had been miscoded. All the remaining patients were contacted through their general practitioners (in all but one practice) and invited to be seen at the NHNN, the general practice, or at home. Those who did not reply were sent a reminder letter and, if they failed to respond, received a reminder telephone call.

4.2.1. Diagnosis. All patients who agreed to participate had a general and neurological interview and examination. I also administered a questionnaire specifically designed to detect signs and symptoms of PSP, MSA, or other atypical parkinsonian disorders. To enable independent diagnostic validation, a video recording of the neurological signs was made if the patient consented. The diagnosis was made according to published criteria (see chapter 3 and below) after review and discussion of each subject with NPQ. 
As the diagnosis in some cases may only become evident after a period of time as new clinical features develop, patients were kept under review using three methods. All patients with a diagnosis of parkinsonism, PSP or MSA received a three monthly questionnaire on the development of atypical features and symptoms of progression (e.g. development of falls). Secondly, the general practitioners were asked at the end of the study whether they had noticed any new atypical features in any of their eligible patients. Finally, patients with either no definitive diagnosis or with atypical features at the first visit, or who developed them during follow-up, were reviewed and reexamined after at least one year. As much information as possible was sought from the general practitioner and other hospitals they had attended on those patients who declined to participate. The same diagnostic criteria were applied to these patients.

4.2.2. Diagnostic criteria. PSP (probable and possible) was diagnosed according to criteria proposed by the National Institute of Neurological Disorders and Stroke (NINDS) and the Society for PSP, Inc. (SPSP; Litvan, 1996a), and MSA (probable and possible) according to the criteria of Quinn (Quinn, 1989; table 3 and 4).

4.2.3. Data analysis. The numbers of patients registered with each general practice by sex and age were obtained from the practice or the responsible Family Health Services Authority. Prevalence day was 1st July 1997. Crude prevalence and age-adjusted rates were calculated by direct standardisation to the 1996 European Population (Eurostat Data Shop). Crude incidence rates were calculated indirectly by dividing the prevalence rates by mean disease duration from previous publications (see below), making the assumptions of a steady state population. $95 \%$ confidence intervals $(95 \% \mathrm{CI})$ were calculated for the crude rates using the Poisson formula and for the standardised rates according to the method of Smith (Smith, 1987).

\subsection{Results}

The total population was 121,608 people, of whom 23,859 were older than 55 , and 14,272 older than 65 years. The screening process identified 679 patients, but 438 patients were excluded after reviewing their records, leaving 241 eligible patients. The reasons for exclusion were a) the use of antiparkinsonian drugs for other indications in 334 patients $(76 \% ; 286$ of whom had been receiving an anticholinergic in association 
with a dopamine antagonist for a psychiatric disorder), b) a known alternative cause of tremor in 51 patients $(12 \%), \mathrm{c})$ drug-induced parkinsonism in 43 patients $(10 \%), \mathrm{d})$ dementia before the onset of parkinsonian symptoms in 5 patients $(1 \%)$, and e) miscoding in 5 patients (1\%). 202 of these patients agreed to be examined ( $84 \%$ participation rate), with 33 patients declining to participate, and 6 patients died before they could be seen. There were no statistically significant differences for sex (59\% vs. $51 \%$ female, $\mathrm{Chi}^{2}$ value $=0.63, \mathrm{p}=0.43$ ) comparing patients who were not with those who were seen, but patients who were not seen were significantly older than those who were seen $(79.5$ years vs. 73.0 years, t-test $p<0.001)$.

170 patients fulfilled the criteria for parkinsonism (137 patients seen, 33 not seen). Five patients fulfilled criteria for probable PSP, and one patient was classified as having possible PSP. Two patients had probable MSA, and one patient qualified for possible MSA. One further patient, who had declined to be seen, was under the care of a movement disorder specialist with a suspected diagnosis of MSA, and fulfilled criteria for possible MSA. Apart from this case, none of the other patients had been previously diagnosed with either of these two diseases (table 6), the previous diagnoses being PD $(n=7)$, small vessel disease $(n=1)$ and no diagnosis $(n=1)$. Only two of the MSA patients and three of the PSP patients had previously seen a neurologist. In addition to these ten patients, another four with parkinsonism and features atypical for idiopathic PD were seen, who did not fulfil criteria for PSP, MSA, or another specific parkinsonian disorder (see below). All but one patient with probable PSP, who was of Asian origin, were Caucasian. There were no cases of corticobasal degeneration or other, rare neurodegenerative disorders.

4.3.1. Prevalence. The crude and age-adjusted prevalence for PSP, if all probable and possible cases were included, was 4.9 per 100,000 (95\% confidence interval 1.8 to 10.7 per 100,000) and 6.4 per 100,000 (95\% confidence interval 2.3 to 10.6 per 100,000), respectively (table 7). If only those with probable PSP were included, the crude and age-adjusted prevalence of PSP was 4.1 and 5.5 per 100,000. If it was assumed that all atypical, not classifiable cases had PSP, the prevalence for PSP would be 8.2 and 11.1 per 100,000, respectively. The crude prevalence for MSA (all probable and possible 
cases), was 3.3 per 100,000 (95\% confidence interval 0.9 to 8.4 per 100,000$)$ and adjusted rate was 4.4 per 100,000 (95\% confidence interval 1.2 to 7.6 per 100,000$)$ (table 7). If only the probable cases were included, the crude and age-adjusted prevalence of MSA were 1.6 and 2.2 per 100,000, respectively. If it was assumed that all atypical, not classifiable cases had MSA, the crude and age-adjusted prevalence for MSA would be 6.6 and 9.0, respectively.

\subsubsection{Gender differences. 116 men (48.1\%) and 125 women (51.9\%) were eligible for} the study. The rates for men and women with probable or possible PSP were almost identical - 5.0 per 100,000 and 4.8 per 100,000 based on 3 male and 3 female cases (relative rate $1.05,95$ confidence interval 0.14 to 7.8 ). For probable and possible MSA, the rates were 1.7 per 100,000 from men and 4.8 per 100,000 for women (relative rate $0.35,95 \%$ confidence interval 0.01-4.3) Among the four patients with atypical, not otherwise classified parkinsonism, one was male.

4.3.3. Incidence. Using survival times of 5.6 years for PSP derived from Litvan et al (Litvan, 1996b) and 5.3 years derived from Bower et al (Bower, 1997), indirect ageadjusted incidence rates were calculated for (probable and possible) PSP of 1.14 and 1.21 per 100,000 per year, respectively. For probable and possible MSA, using survival times of 9.5 derived from Wenning et al (Wenning, 1994a) and 8.5 derived from Bower et al (Bower, 1997), age-adjusted incidence rates were obtained of 0.46 and 0.52 per 100,000 per year, respectively.

\subsection{Discussion}

To my knowledge, this is the first large population-based prevalence study that has specifically attempted to measure the frequency of PSP or MSA using currently accepted diagnostic criteria and with a period of follow-up to increase diagnostic accuracy. The methods in this study have resulted in higher prevalence rates (PSP 6.4 per 100,000 , MSA 4.4 per 100,000) than have been previously reported. The results also illustrate the importance of community-based studies as none of the PSP cases and only one of the MSA cases were previously diagnosed. Initially, wide inclusion criteria were used so that all potential cases of parkinsonism could be identified. Not only were all patients examined with a particular view on detection of features indicating an atypical parkinsonian syndrome, but almost all also had a video recording taken for 
discussion and diagnostic confirmation. Twenty three patients with any atypical feature(s) were reviewed after a year follow-up period, two (9\%) of whom received a new diagnosis (one with probable PSP and one with atypical parkinsonism). These methods identified more patients than would have been found using more conventional methods.

The frequency of PSP and MSA has been estimated from a German door-to-door study of 1190 people aged 65 years or over. (Trenkwalder, 1995). The investigators did not identify any cases of PSP but found 3 cases of MSA and 7 cases of PD. Their prevalence rate for MSA (252 per 100,000, 95\% confidence interval 52 to 734 per $100,000)$ is very much greater among people older than 65 years than the equivalent rate in this study (28 per 100,000, 95\% confidence interval 8 to 72 per 100,000). In contrast, the prevalence for $\mathrm{PD}$ in this population (588 per 100,000, 95\% confidence interval 236 to 1212) is less than half the usual rate in Caucasian populations for patients over the age of 65 years (Tison, 1994). A door-to-door survey should identify not only cases who have, but also those who have not, sought medical help, so this might partially explain the difference. However, given the ease of access to primary care in the United Kingdom and the rapid progression of MSA, it is more likely that, given the wide confidence intervals, the extraordinary high prevalence rate of MSA in the German study is due to chance. In contrast, a large prevalence study, which specifically focused on PSP, but only included pre-diagnosed patients (Golbe, 1988), found a much lower prevalence rate of 1.4 per 100,000, which may be accounted for by failure to include mis- or undiagnosed patients. No comparable data on the prevalence of MSA derived from large population samples are available.

A number of studies on the prevalence of $\mathrm{PD}$, which specifically excluded patients with PSP and MSA, have reported data that enable presumptive calculation of their prevalence, even though one is uncertain of which diagnostic criteria were used. Wermuth et al (Wermuth, 1997) found a prevalence of PSP and MSA of 4.6 and 2.3 per 100,000, respectively, in a study of PD on the Faroe Islands with a population of 43,000. These results are similar to the findings in this study and given the small population of the Islands it is likely that all cases were identified. Chio et al (Chio, 
1998) found a prevalence of 3.2 and 4.9 per 100,000 for PSP and MSA, respectively, examining all patients on antiparkinsonian drugs in a study in Northwestern Italy covering a population of 61,830 . The prevalence data among patients above the age of 55 from the study by de Rijk et al in Rotterdam (De Rijk, 1995; 14.3 and 28.7 per 100,000 for PSP and MSA, respectively) are also comparable to those in the patients in this study above the age of 55 (25.1 and 16.8 per 100,000, respectively). However, small numbers (overall population of 6,969) once again make comparison difficult. Additionally, the degree of diagnostic certainty in these studies, which were mainly designed to detect idiopathic $\mathrm{PD}$, is unclear.

There are only a few reports on the incidence of PSP and MSA, yielding rates of between 0.3 to 1.1 (Bower, 1997; Mastaglia, 1973; Radhakrishnan, 1988; Rajput, 1984) per 100,000 per year for PSP and 0.6 per 100,000 per year for MSA (Bower, 1997). These findings are fairly comparable to the results of this study but incidence was measured indirectly so one must be cautious in making comparisons.

The frequency of PSP and MSA in this study as a proportion of all cases with parkinsonism was about half that seen from autopsy series from Parkinson brain banks. It is possible that cases who had not yet developed atypical features were underascertained. However, patients with atypical parkinsonian disorders are more likely to come to autopsy than those with idiopathic PD (Maraganore, 1998), and PSP and MSA are therefore likely to be over-represented in autopsy series.

Prevalence rates of PSP were similar for men and women in this study, in contrast to studies finding a higher incidence of PSP in men (Steele, 1964; Bower, 1997). It is possible that male cases are more likely to be ascertained from specialist centres (Rybicki, 1995). Alternatively, these discrepant observations may be reconciled if men with PSP die more rapidly than women. The results of this study do not support hypotheses of occupational risk factors in men or hormonal protection in women. MSA was more frequent in women, but due to the small numbers no firm conclusions can be drawn from this finding. Both diseases increased with increasing age, but fewer patients were found in the age-group above 80 years. This may indicate a true declining 
prevalence of these disorders, but may also be due to underascertainment, since a parkinsonian syndrome is less likely to be suspected in older patients (De Rijk, 1995) and older patients were more likely to decline participation. In addition, the increasing prevalence of concomitant dementia with increasing age may have lead to exclusion of cases of PSP (which can cause dementia) in this oldest age group if it began before the onset of parkinsonism. Last, it may also purely be a chance finding, as the numbers were small in this oldest age group.

Despite efforts to maximise case ascertainment, the prevalence rates for both diseases are probably underestimated for the following reasons. Firstly, despite a good participation rate (84\%), cases amongst non-participants could have been missed. The notes of all such patients were checked and in one patient the recorded clinical features were sufficient to make a diagnosis of possible MSA. Secondly, in some cases the correct diagnosis will only become evident at post-mortem, as demonstrated by the retrospective analysis of clinical features of patients with pathologically confirmed PSP and MSA (Hughes, 1992). Thirdly, I only ascertained cases who had been seen in primary care, so that early cases may have been missed. This is more likely to be a problem for PD, particularly in elderly patients, than for PSP or MSA, as these disorders progress more rapidly and are severely disabling. Finally, potential cases were seleced from patients with parkinsonism as most patients with PSP (Litvan, 1996a) and MSA (Wenning, 1994a) develop parkinsonian features during the course of the disease. However, patients with early PSP or MSA presenting only with falls, ataxia or orthostatic hypotension may not have been detected.

With the demographic increase in the elderly population, parkinsonian movement disorders will increase in relative importance. Whilst both PSP and MSA are much less frequent than $\mathrm{PD}$, their prevalences have been under-estimated and patients in the general population are often undiagnosed. This has important implications for treatment, prognosis and providing community care, particularly since these diseases are more disabling than PD and respond much less well to antiparkinsonian medications. Until better diagnostic tests are available, clinical studies must use rigorous methods with patient follow-up to provide more realistic estimates of disease frequency. 
Chapter 5. The population prevalence of Parkinson's disease and parkinsonism in London

\subsection{Background}

Idiopathic Parkinson's disease (PD) is one of the most frequent neurological disorders, particularly in the elderly. Estimates of its prevalence have however varied widely between studies and countries ranging from 10 (Nigeria) to 405 (Uruguay) affected patients per 100,000 people in the general population (Zhang, 1993). The interpretation of these differences is difficult as methodological (e.g. the use of different diagnostic criteria, varying methods of case ascertainment and different age-distributions) rather than true geographical differences account for at least part of them. The few previous studies on the prevalence of PD in the United Kingdom have yielded prevalence rates of 108 to $164 / 10^{5}$ with little change over the last decades (Sutcliffe, 1995), and none of them have been conducted in the London area.

This study investigated the prevalence of $\mathrm{PD}$ and other parkinsonian syndromes in the London area using a population-based approach with special emphasis on distinguishing different parkinsonian syndromes.

\subsection{Patients and methods}

Patients whose onset of parkinsonian symptoms was within 6 months of at least 6 months of treatment with dopamine receptor blocking drugs, and patients who had developed dementia before the onset of parkinsonism were not contacted, but their detailed medical records were extracted. The remaining patients were contacted through their general practitioners (in all but one practice) and invited to be seen at the NHNN, at their general practice, or at home.

5.2.1. Diagnostic criteria. Parkinsonism was diagnosed if bradykinesia and at least one other cardinal sign (resting tremor, rigidity or postural instability) were present. PD was diagnosed according to the UK Parkinson's Disease Society Brain Bank criteria (Gibb, $1989 \mathrm{~b}$ ) with the exception that an isolated positive Babinski sign, for instance in an elderly patient with otherwise typical PD was not considered to invalidate the diagnosis. In addition, patients with isolated classical resting tremor only were included as having 'possible Parkinson's disease'. Multiple system atrophy (probable and possible) was 
diagnosed according to Quinn (Quinn, 1989), and progressive supranuclear palsy (probable and possible) according to criteria proposed by the National Institute of Neurological Disorders and Stroke (NINDS) and the Society for PSP, Inc. (SPSP; Litvan, 1996a). Vascular parkinsonism was diagnosed when there was presence of at least two of the following: history of previous strokes, abrupt onset with stepwise progression, hypertension, a wide-based gait with small steps, cognitive decline, pseudobulbar or pyramidal signs. Drug-induced parkinsonism (DIP) was diagnosed if the onset of parkinsonian symptoms was within 6 months of at least 6 months treatment with dopamine receptor blocking drugs. Patients with DIP, and those with dementia before the onset of parkinsonian symptoms, were not seen, but their details were extracted from the records of the general practitioner. In patients with DIP, parkinsonism had to be well documented and still present on prevalence day. Patients in whom only tremor on treatment with a dopamine receptor blocking drug was noted were not included.

5.2.2. Data analysis. Prevalence day was July 1st, 1997. All patients seen who had died before prevalence day, or moved away from the study area were excluded. The numbers of patients registered with each general practice by sex and age were obtained from the practice or the responsible Health Authority. We calculated crude prevalence rates for this population, and age-adjusted rates by direct standardisation to the 1997 United Kingdom Population. In addition, the results were also adjusted to the European population, including all member states of the European Union at that time. The $95 \%$ confidence intervals $(95 \% \mathrm{CI})$ around age specific rates were calculated assuming the Poisson distribution (Gardner, 1992) whilst the method of Smith (Smith, 1987) was used for the $95 \%$ CIs around the directly standardised rates.

\subsection{Results}

235 patients fulfilled the criteria for parkinsonism, including DIP, vascular parkinsonism and parkinsonism in dementia, (148 patients of those who were, and 87 of those who were not seen). Probable PD was diagnosed in 152 patients, and possible PD in four. Three patients had developed parkinsonism following central ( 2 patients) or peripheral trauma (1 patient), and one following intoxication with carbon monoxide. However, in 
all 4 patients, the syndrome was slowly progressive, asymmetric, responsive to levodopa, and also otherwise characteristic for PD. We therefore considered these patients to have PD.

17 patients were diagnosed as having vascular parkinsonism and 43 had drug-induced parkinsonism. Five patients were classified as having parkinsonism with onset after the development of dementia, and 14 patients had atypical parkinsonism, including MSA, PSP and atypical not classifiable parkinsonism (only one of whom was not seen). No cases of postencephalitic parkinsonism or corticobasal degeneration were found. The clinical characteristics of the different groups are given in table 8.

5.3.1. Prevalence. The crude and age-adjusted prevalence figures for $\mathrm{PD}$, if all probable and possible cases were included, were 128 (95\% CI 109 to 150$)$ and 168 (95\% CI 142 to 195$)$ per 100,000 , respectively. The crude and age-adjusted prevalence rates for primary parkinsonism, including atypical parkinsonism were 140 (95\% CI 120 to 162) and 184 (95\% CI 156 to 211$)$ per 100,000, and that of all types of parkinsonism, including DIP, vascular parkinsonism and parkinsonism following dementia were 193 (95\% CI 169 to 220) and 254 (95\% CI 222 to 287) per 100,000, respectively. Ageadjustment to the standard European population gave prevalence rates per 100,000, for PD of 121 (95\% CI 101 to 141$)$, primary parkinsonism of 133 (95\% CI 112 to 154), and for all types of parkinsonism of 186 (95\% CI 161 to 211$)$, respectively.

5.3.2. Age-and sex-specific rates. The prevalence of $\mathrm{PD}$ as well as of parkinsonism as a whole was similar in men and women, with a slightly higher prevalence in men in all ages (table 9). There were two patients with PD currently below the age of 40 . The prevalence of PD increased with advancing age from an age-specific rate of $0.008 \%$ between age 30 and 40 to $1.3 \%$ in those above the age of 80 without a decline in the oldest patient group. This was also true for vascular parkinsonism and post-dementia parkinsonism (table 10). However, the prevalence of atypical parkinsonism showed an inverted U-shape pattern, with a fall in the higher age-group and the rates for DIP plateaued after 60 years of age. There were no patients with vascular parkinsonism or parkinsonism following dementia below the age of 60 . 


\subsubsection{Relative frequency of parkinsonian subtypes among patients with parkinsonism}

Among all patients with parkinsonism, the patients with probable and possible PD represented $66 \%$, those with atypical parkinsonism $6 \%$, those with vascular parkinsonism $7 \%$, those with drug-induced parkinsonism $18 \%$, and those with parkinsonism following dementia $2 \%$ (see table 11 ).

\subsection{Discussion}

\subsubsection{Comparison with findings from other UK studies}

In this study, we found an age-adjusted prevalence rate of 168 per 100,000 for PD in the area of London. There was a slightly higher rate in men than in women, and the agespecific prevalence increased consistently with age, without decline in the highest age group. This pattern is consistent with door-to-door surveys, which have demonstrated that a decline in prevalence for the oldest age group is due to ascertainment bias. Our prevalence rate is similar to that of the previous UK studies, which yielded prevalence rates of 164 reported from Aberdeen in 1984, and 121 reported from Northamptonshire in 1992 (figure 1). Only 2 out of the 20 age-specific rates have $95 \%$ confidence intervals that do not overlap with the pooled age-specific rate. The survey by Brewis and colleagues found a relatively lower rate for patients aged over 79 years and Mutch and colleagues had a relatively higher rate than the other studies for this age group. All the studies have used fairly similar diagnostic criteria (bradykinesia plus one of the other classical features) and patients with DIP and vascular parkinsonism were excluded. The three most recent surveys have used more thorough methods of case ascertainment and confirmation of diagnosis with examination. It is likely that Brewis and colleagues relied predominantly on hospital-based cases although seeking cases in the community, and therefore underascertained elderly cases. The study by Mutch and colleagues actively searched for elderly subjects resident in nursing homes. This may explain their higher prevalence rate for the over eighties age group.

\subsubsection{Comparison with findings from other studies world-wide}

Prevalence rates between different studies show wide variations (Zhang, 1993), but interpreting these differences is difficult owing to variable methodology. Thus, the lowest prevalence rates were found in studies relying on death certificates (Ben-Shlomo, 
1993; Vanacore, 1992), and the highest in population-based studies, which report a frequency of PD between 3 and 4\% in the population over 80 years (Bharucha, 1988; Morgante, 1992; de Rijk, 1997; de Rijk, 1995; Tison, 1994; Wang, 1996). Moreover, the use of different diagnostic criteria leads to markedly differing results between studies, as the difference between the prevalence rate of PD and of all parkinsonism in this study illustrates. Similarly, the crude prevalence rate in a door-to-door study in Sicily was 372 for all kinds of parkinsonism and 257 for PD alone. Additionally, the diagnosis of PD and its differentiation from other subtypes of primary parkinsonism is conspicuously difficult, and the rate of misdiagnosis even at death has been as high as $24 \%$ in the 1980 s in the UK, but is probably lower today, partly as a result of the publication of this study (Hughes, 1992). Furthermore, crude prevalence rates, even if they were created with identical methods, cannot be compared between populations of different population structures. Thus, the crude prevalence rates of 257 and 328 per $10^{5}$ found in the door-todoor studies in Sicily and Bombay, for instance, fall to 194 and 148 per $10^{5}$ adjusted to the 1970 US population, respectively (Zhang, 1993). Likewise, studies only assessing elderly populations (Schoenberg 1985, Tison, 1994) yield considerably higher results than those including all ages. Therefore, comparisons can only be made either after adjustment to the same population, or the age-specific rates are compared directly for each age-group. We here present the prevalence rates age-adjusted to the UK and standard European populations, and the age-specific prevalence rates of this and other studies (figure 1 and 2).

Provided similar methodology was employed, many studies world-wide have arrived at comparable age-specific prevalence rates (figure 2). The age-specific prevalence rates of this study are among the highest, with the exception of the door-to-door studies. These are probably the most accurate of prevalence studies, and generally yielded higher results with a substantial proportion of newly diagnosed patients. However, the use of different diagnostic criteria hampers a comparison even between these studies. Only one door-to-door study has been conducted, to my knowledge, with neurological expert diagnosis at screening level owing to the immense costs of such a study (Wang, 1996).

\subsubsection{Potential underascertainment}


Between 12\% (de Rijk, 1995) and 78\% (Wang, 1996) of patients are newly diagnosed in door-to-door studies, and are missed in studies relying on medical records. Although as complete case ascertainment as possible was attempted by including every patient who had ever been suspected of a parkinsonian syndrome, had received antiparkinsonian drugs, or had been noticed to have a tremor (with onset after age 50), this study was not a door-to-door survey, and patients who have not come to medical attention would not have been included. In the EUROPARKINSON collaboration, the proportion of newly diagnosed community cases varied between $11 \%$ (France) and 13\% (Holland up to $52 \%$ (Pamplona, Spain; de Rijk, 1997). We suspect that the UK health care system resembles the Dutch health care system in terms of quality and access and therefore the proportion of 'missed' cases would be around $10-20 \%$.

\subsubsection{Diagnostic accuracy}

A certain degree of uncertainty remains in those who were not personally examined. Their notes were examined in detail, and the same criteria applied to these patients. However, as this documentary data was less complete and thorough than in the patients who were seen, it is possible that any patients with atypical parkinsonism may have been misclassified as PD.

\subsubsection{Other causes of a parkinsonian syndrome}

Only 66 percent of all patients with parkinsonism suffered from PD. The other 34 percent had secondary parkinsonism including 6 percent with other types of primary parkinsonism. This percentage is higher than generally assumed (Rajput, 1984; de Rijk, 1997), but less than recent data from Rochester, Minnesota (Bower, 1999), where 41 percent of incident cases of parkinsonism had a diagnosis other than PD, but $17 \%$ of cases were classified as parkinsonism unspecified. The figure in this study might also have been even higher if all patients with drug-induced parkinsonism or tremor were seen, as the most likely cause of tremor in patients taking dopamine receptor blocking agents is drug-induced parkinsonism. In addition, it is possible that not all patients with DIP, parkinsonism following dementia, or vascular parkinsonism were correctly coded by every general practitioner. For this reason, and as the main purpose of this study was to determine the prevalence of PD and atypical parkinsonism, these patients were not 
personally examined. However, it is likely that the rate of drug-induced parkinsonism and vascular parkinsonism is higher. Overall, the prevalence of atypical parkinsonism was approximately $10 \%$, which has important implications for treatment trials, where these patients should be excluded due to the different response to medication and their different natural history with more rapid progression and worse prognosis.

\subsection{Conclusion}

We conclude that the prevalence of PD in the London area is similar to that reported from other parts of the UK. However, the total number of patients with parkinsonism, including atypical parkinsonism, DIP, vascular parkinsonism, and parkinsonism following dementia, is considerably higher. Prevalence rates increase with advancing age without a decline in the oldest age group, and there was a slightly higher prevalence in men. Assuming that $10-20 \%$ of patients had escaped medical attention, the prevalence of PD in London is estimated as approximately 200 per 100,000. 
Chapter 6. Accuracy of the clinical diagnosis of Parkinson's disease in the community

\subsection{Background}

Pathological examination of brains of patients with a clinical diagnosis of Parkinson's disease reveals a different diagnosis in up to $35 \%$ of cases (Rajput, 1991; Hughes, 1992; Ansorge, 1997; Litvan, 1998). Retrospective application of strict clinical criteria increases the likelihood of correct diagnosis (Hughes, 1992), although a proportion of patients who do not have PD still fulfil clinical criteria for PD in such a retrospective analysis. Conversely, patients with PD may be misdiagnosed as having other parkinsonian disorders (Litvan, 1998), or not be recognised to have parkinsonian features (Schoenberg, 1985). In the absence of a biological marker for $\mathrm{PD}$, the only certain means of diagnosis at present is the finding of depletion of brain stem pigmented neurons with Lewy bodies at pathological examination. However, pathological examination is only possible in a minority of patients with parkinsonism, and pathological case series are prone to referral bias and probably overrepresent atypical cases (Maraganore, 1998). In order to determine the accuracy of a diagnosis of PD in the general, unselected, population of patients with a parkinsonian syndrome, we applied the clinical 'goldstandard' of strict published criteria to all patients identified in this population-based study on the prevalence of PD.

\subsection{Methods}

The diagnosis of non-parkinsonian tremor included various types of tremor such as essential, dystonic and enhanced physiological tremor, as the distinction of these syndromes is still a matter of debate (Chouinard, 1997). Statistical analysis consisted mainly of calculating rates and proportions.

\subsection{Results}

134 patients ( $66 \%$ of 202 participants) had received a diagnosis of parkinsonism (131 of $\mathrm{PD}$, one of atypical parkinsonism and two of vascular parkinsonism). Ten additional patients (5\%) had been prescribed antiparkinsonian drugs for parkinsonian symptoms without a further specified diagnosis. 56 patients $(28 \%)$ had been noted to have a tremor with onset after age 50 without having been suspected of having 
parkinsonism, and two patients (1\%) were referred to Professor Quinn for diagnostic purposes without a previous diagnosis (table 12).

\subsubsection{Patients with a previous diagnosis of $P D$}

The diagnosis of probable PD was confirmed in 109 patients with this diagnosis $(83 \%)$, including three patients $(2 \%)$ in whom atypical features were found, but were insufficient to discard the diagnosis of PD. Two additional patients $(2 \%)$ were found to have possible PD. However, in 20 patients (15\%) the diagnosis of PD was unequivocally rejected. The alternative diagnoses were non-parkinsonian tremor in 4 patients $(3 \%)$, vascular parkinsonism in $6(5 \%)$, progressive supranuclear palsy (PSP) in 4 (3\%; probable in 3 and possible in one), and of multiple system atrophy (MSA) in 3 patients (2\%; probable in 2 and possible in one). Two patients received a diagnosis of idiopathic torsion dystonia, and one of dementia without parkinsonism (who was recorded as having PD and dementia not clearly starting before parkinsonism). When only those patients who had seen a consultant neurologist at some point in the past ( 72 patients, $55 \%$ ) were considered, the diagnosis was changed from Parkinson's disease to a different diagnosis in $8 \%$ (table 13).

\subsubsection{Patients with a previous diagnosis other than Parkinson's disease}

Among all patients seen, two were referred for diagnostic purposes without a previous diagnosis, and 69 patients (34\%) had a previous diagnosis other than PD. Among these, $81 \%$ had been given a diagnosis of non-parkinsonian tremor, $3 \%$ of vascular parkinsonism, $1 \%$ of atypical parkinsonism, and $14 \%$ had been prescribed an antiparkinsonian drug for parkinsonian features without a specific diagnosis. Thirteen of the patients with different diagnoses (19\%) and the two patients referred for diagnostic purposes fulfilled strict clinical criteria for PD. In two additional patients who had a previous diagnosis of non-parkinsonian tremor, a diagnosis of 'possible PD' was made (3\%; table 14). If only patients who had a some point in the past seen a consultant neurologist (39\%) were considered, the diagnosis was changed to $\mathrm{PD}$ in $11 \%$ of them.

\subsubsection{Sensitivity, specificity and predictive value of a previous diagnosis of $P D$} The definitions of sensitivity, specificity, positive and negative predictive value are given in the appendix. Sensitivity and specificity are independent of the sample size, 
whereas predictive values change with the size of the study population. They are therefore prone to selection bias in clinic-based or pathological studies, but are also dependent of the size and type of the investigated study sample (e.g. all patients with tremor or all patients with parkinsonism). These parameters were calculated for the overall sample excluding the patients referred for diagnosis (both of whom had PD), as well as separately for the subgroups of patients identified in the screening phase as having tremor or as having parkinsonsism.

The sensitivity of a clinical diagnosis of probable PD in the overall sample (patients identified through an initial diagnosis of parkinsonism, of tremor with onset after age 50, or identified through previous prescription of antiparkinsonian drugs) was $89.3 \%$ and the specificity $71.8 \%$. The positive and negative predictive values of a previous clinical diagnosis of $\mathrm{PD}$ were 83.2 and $81.2 \%$. If the patients with possible PD were included in the group of patients with PD, the sensitivity was $88.1 \%$, the specificity $73.0 \%$, and the positive and negative predictive values were 84.7 and $78.3 \%$.

Among patients identified because of tremor starting after age 50, the sensitivity, specificity and positive and negative predictive values were 64.0, 97.9, 94.1 and $83.9 \%$. If patients with possible PD were included in the group of patients with PD these values were $60.7,100,100$ and $80.4 \%$.

Among patients identified through a previous diagnosis of parkinsonism the sensitivity, specificity and positive and negative predictive values for a diagnosis of probable PD were 97.3, 31.3, 83.2 and 76.9\%, and when patients with possible PD were included $97.4,33.3,84.7$ and $76.9 \%$.

\subsubsection{Comparison of patients in whom a diagnosis of $P D$ was maintained or rejected}

Patients in whom a diagnosis of $P D$ was confirmed had more severe disease as measured by the Hoehn and Yahr stage $(p<0.05)$, more often had a tremor at rest $(\mathrm{p}<0.01)$ or a classical pill-rolling tremor $(\mathrm{p}<0.01)$ and more frequently reported a good initial and sustained response to levodopa (both $\mathrm{p}<0.001$ ) than those in whom it was rejected.

Patients in whom the diagnosis was changed to non-parkinsonian tremor had no other parkinsonian features like rigidity, bradykinesia, hypomimia or monotonous speech. They also reported falls significantly less frequently $(\mathrm{p}<0.01)$ and had higher minimental scores $(p<0.05)$. Those in whom the diagnosis was changed to atypical 
parkinsonism (multiple system atrophy or progressive supranuclear palsy) had more severe akinesia $(p<0.05)$, rigidity $(p<0.01)$ and postural instability $(p<0.05)$, reported less frequently an initially or currently good response to levodopa (both $\mathrm{p}<0.01$ ), but more frequently incontinence $(\mathrm{p}<0.01)$, and had additional features incompatible with PD. Those in whom the diagnosis was changed to vascular parkinsonism were older than those in whom a diagnosis of PD was confirmed $(\mathrm{p}<0.05)$, had a higher number of smoking years $(\mathrm{p}<0.01)$, more frequently had gait difficulties as their first complaint $(p<0.05)$ and had more severe postural instability $(p<0.001)$ than those in whom PD was confirmed, and never had rest tremor.

\subsubsection{Comparison of $P D$ patients in whom a diagnosis of $P D$ was or was not} previously made

Patients in whom a diagnosis of PD was previously made had a longer disease duration $(p<0.05)$, and higher disease severity $(p<0.01)$ with more severe akinesia $(\mathrm{p}<0.01)$, postural instability and rigidity (both $\mathrm{p}<0.001)$ than those in whom the diagnosis was not made before. They were also more likely to be depressed $(p<0.05)$, to have experienced dyskinesias $(\mathrm{p}<0.05)$, and to live alone or with their family than in a nursing home $(\mathrm{p}<0.05)$.

\subsection{Discussion}

The results of this study suggest that $\mathrm{PD}$ in the population is frequently confused with other disorders. The main areas of diagnostic difficulty concern distinction from other types of isolated, late onset tremor, vascular parkinsonism and atypical types of parkinsonism, which are often mistakenly diagnosed as PD. On the other hand, patients with PD are sometimes not recognised as having this disorder, particularly those with mild disease and a relatively isolated tremor. The rate of underdiagnosis is likely to be even higher as only patients who had already come to medical attention with tremor or parkinsonian features were included. Some patients not meeting the screening criteria, particularly elderly people with akinetic-rigid parkinsonism without tremor would have been missed in this study, if postural instability and other parkinsonian features were attributed to old age. In door-to-door studies on the prevalence of $\mathrm{PD}$, the percentage of undiagnosed patients ranged from 12 to $60 \%$ (Schoenberg, 1985; De Rijk, 1995). Wide screening criteria were employed in this study in order to detect as many patients with a parkinsonian 
syndrome as feasible. For this reason, and due to the comparatively easy access and high density of neurologists in the study area of London, the rate of underdiagnosis is likely to be relatively low and comparable to that of $12 \%$ found in a recent door-todoor study with similar study design in the Netherlands (De Rijk, 1995).

As may have been expected, $\mathrm{PD}$ was more often recognised in patients with greater disease severity, who had had the disease for longer and had already developed complications such as depression and dyskinesias, and were more likely to live in a nursing home than alone or with their family.

The accuracy of a diagnosis of PD has been conspicuously poor in the few studies assessing patients with this clinical diagnosis. When compared to pathological diagnosis, the rate of false positive diagnosis of PD was $24 \%$ in one study of 100 patients with a clinical diagnosis of PD (Hughes, 1992). This rate improved to $18 \%$ when strict clinical criteria were used retrospectively. In a second, later study the same group reported in abstract form that the rate of misdiagnosis in a series of 100 patients who had died with a diagnosis of PD had dropped to $16 \%$ (Ansorge, 1997). In another study of 43 patients with a clinical diagnosis of PD, the rate of correct diagnosis at the initial visit was only $65 \%$ compared to pathological diagnosis, but rose to $76 \%$ at the final visit (Rajput, 1991). A more recent study of 29 patients with Lewy body disease ( 15 with PD and 14 with diffuse Lewy body disease) found that only $60 \%$ of patients at the first and $77 \%$ at the last visit in fact had Lewy body disease. For PD alone, the correct diagnosis of PD was made in 46\% and $64 \%$ at the initial and final visit, respectively (Litvan, 1998). However, this high rate of misdiagnosis is probably overestimated as the total study sample of 100 patients was highly unrepresentative of the overall population of patients with parkinsonism, and also included patients with PSP, MSA, corticobasal degeneration, postencephalitic parkinsonism, Pick's disease, Alzheimer's disease with exrapyramidal features, Creutzfeldt Jakob disease, vascular parkinsonism and Whipple's disease. Samples from brain banks and specialist clinics are similarly likely to overrepresent atypical disorders due to the referral bias inherent in such samples (Maraganore, 1998). Nevertheless, prevalence studies of Parkinson's disease, which employed strict clinical diagnostic criteria also yielded rates of false positive diagnoses ranging from 14.5 to $44 \%$, the commonest alternative diagnoses being essential tremor, vascular parkinsonism, dementia and drug-induced parkinsonism (Marttila, 1976; Sutcliffe,1995; Mutch, 1986a). In a recent study 
assessing the accuracy of diagnosis in patients with presumed $\mathrm{PD}$, using the same criteria for PD (Gibb, 1989b) as in this study, only 53\% of 402 patients were clinically confirmed to have PD (Meara, 1999). Patients with drug-induced parkinsonism and dementia before the onset of parkinsonism, who were excluded in this study, constituted $12 \%$ and $4 \%$, respectively, and patients who did not fulfil full criteria for probable PD, which would have been labelled 'possible PD' in this study, were included in the group of 'unspecified parkinsonism'. Thus, if these cases are excluded, the rate of false positive diagnosis was similar in both studies. However, in the study cited (Meara, 1999), the diagnosis was more frequently changed to essential tremor (12\%), which was the final diagnosis in only $3 \%$ of patients previously diagnosed as PD in this study. A possible explanation for this finding may be earlier referral to neurology clinics and earlier diagnosis in this study due to the high density and relatively easy access to neurology departments in London.

A false positive diagnosis of $\mathrm{PD}$ was made predominantly in patients with other neurodegenerative causes of parkinsonism, such as multiple system atrophy and progressive supranuclear palsy, and in patients with vascular parkinsonism. This is in agreement to what has been found in pathological studies, where approximately $6 \%$ of incorrectly diagnosed patients had PSP, $5-9 \%$ had MSA and 3\% had vascular parkinsonism (Rajput, 1991; Hughes, 1992). As patients with dementia who later on developed parkinsonism were excluded, the rate of false positive diagnosis of $\mathrm{PD}$ in patients with Alzheimer's disease, which represented 2-6\% in pathological studies, is likely to be smaller in this sample. Likewise, patients who had used neuroleptic drugs within 6 months of the onset of symptoms were excluded a priori, and the rate of misdiagnosis of drug-induced parkinsonism was therefore lower. The accuracy of diagnosis of PD in the general population in this study thus reflects the pattern of diagnostic difficulties in the clinico-pathological studies.

In order to improve the accuracy of diagnosis, this study was specifically designed to detect atypical features, which is not possible in a retrospective analysis of patients coming to postmortem examination, and included video-recording of the patients, and obtaining follow-up information over more than one year with review of all patients with uncertain diagnosis at the end of the study. The accuracy of clinical diagnosis in this study is therefore likely to be relatively high. However, some patients do not develop atypical features even until death and can therefore not be unequivocally diagnosed during life. When using the same diagnostic criteria that 
were applied in this study, Hughes et al still found a rate of misdiagnosis of PD in $18 \%$ at post mortem examination (Hughes, 1992). Although in a later study by the same group the rate of misdiagnosis was lower (Ansorge, 1997), and despite the prospective approach of this study, at least another $10 \%$ of patients diagnosed with PD are likely to suffer from a different disorder.

Features helpful for the diagnosis of PD were the presence of a classical pillrolling tremor, a good and sustained response to levodopa and advanced disease with postural instability. Features suggestive of a diagnosis of non-parkinsonian tremor were the lack of rigidity, bradykinesia, cognitive decline or falls, whereas patients with vascular parkinsonism were older, had a longer history of smoking, more frequent gait difficulties, less frequently tremor, and more severe postural instability. In addition, this diagnosis was based on the presence of a wide-based small-stepped gait, history of previous strokes, abrupt onset with stepwise progression, hypertension, cognitive decline, pseudobulbar or pyramidal signs. Suggestive of atypical parkinsonism, on the other hand, were a more severe parkinsonian syndrome, greater postural instability, a poor initial or current response to levodopa, incontinence, and additional features incompatible with PD. These features, if not already part of the diagnostic criteria, may further help making a diagnosis in a patients with possible parkinsonism.

Overall, at least $15 \%$ of patients with a diagnosis of $\mathrm{PD}$ in the population did not fulfil strict clinical criteria for PD, and approximately $20 \%$ of patients with $\mathrm{PD}$ who have already come to medical attention were not diagnosed as such. The application of strict clinical criteria helps to identify patients with idiopathic PD, PSP and MSA, and aids their differentiation from vascular parkinsonism, drug-induced parkinsonism and non-parkinsonian tremor. 


\section{Chapter 7: The clinical features and natural history of parkinsonian disorders}

Only the group of patients with Parkinson's disease was large enough to allow further analysis of clinical features. I will therefore discuss the clinical characteristics and subgroups of this group and only describe the clinical characteristics of the patients with atypical parkinsonian disorders.

\subsection{Clinical characteristics of patients with progressive supranuclear palsy and multiple system atrophy}

The clinical characteristics of the patients with PSP and MSA in this study are similar to reported case series. Disease duration of the probable and possible PSP patients ranged from 1 to 10 (mean 4.4) years, and of the MSA patients from 1 to 7 (mean 3.3) years. All patients with possible or probable PSP or MSA had parkinsonism and a poor response to levodopa. All patients with PSP had prominent postural instability and falls, as well as a vertical supranuclear gaze palsy. All patients with MSA had urinary symptoms, but none had cerebellar signs. Orthostatic faintness was experienced by all patients with probable or possible MSA, one of whom (with additional diabetes) had recurrent syncope. Two patients with PSP and one with MSA had pyramidal signs. Cognitive impairment, by history and as assessed by a mini mental test (cut-off score 24 out of $30 ; 27$ ), was present in 3 of the 6 patients with PSP. Among the four patients with atypical features that did not suffice to exclude them from the group of patients with Parkinson's disease, 2 had a poor or declining response to levodopa, 2 had pyramidal signs, 2 early and prominent falls, and 3 urinary dysfunction (severe in one and mild in two). The characteristics and atypical features in these patients and of all patients with atypical parkinsonism are shown in table 6. 


\subsection{The clinical features of Parkinson's disease in a population-based sample}

\subsubsection{Background}

The clinical features and natural history of PD have been well described in clinic-based samples (Hoehn, 1967; Poewe, 1998). However, clinic-based samples may not be representative of the overall population of patients with $\mathrm{PD}$, as they may overestimate e.g. the proportion of younger or more severely affected patients, and thus under- or overestimate disability and disease burden in the general population. As a result of such a bias, the rate of clinical features and complications, and the factors determining disability may be different in clinic-based samples than in the community, and the rate of progression may be skewed towards more rapid disease. I therefore analysed the prevalence of clinical features and complications of $\mathrm{PD}$, and their impact on disability in this unselected population of patients with $\mathrm{PD}$, and analysed the rate of progression in these patients.

\subsubsection{Patients and methods}

All patients diagnosed with probable (n-124) or possible $(n=4)$ idiopathic PD in this study were included. The tremor-dominant subtype of PD was defined as patients with a ratio of tremor to bradykinesia score (bradykinesia, rigidity and postural instability subscores from the UPDRS motor scale and history) $>0.5$, and the akinetic-rigid subtype as patients with a ratio of $<0.5$ (modified from Jankovic, 1990).

\subsubsection{Statistical analysis}

In addition to the above described methods of statistical analysis, the rate of progression was calculated by dividing Hoehn and Yahr stage at the time of the examination by the duration of symptoms in years. Subjective response to levodopa was divided in poor or none $(<25 \%)$, moderate $(26-50 \%)$, good $(51-75 \%)$, and excellent $(>75 \%)$. Statistical significance was accepted when $\mathrm{p}<0.05$. Multiple regression analysis was performed to examine the factors influencing disability.

\subsubsection{Results}


67 patients (52.3\%) were male and $61(47.7 \%)$ female. The average age was 72 (SD 10.9) and average age at onset 66 (SD 14.3) years. Average disease duration was 6.0 (SD 4.6) and average duration of treatment was 4.8 (SD 4.8) years. 19 (15\%) patients were younger than 60 and $29(23 \%)$ older than 70 years at the time of the investigation. $3 \%$ of patients had had disease onset before the age of 40 , and 22 after the age of 70 . Disease duration was shorter than 5 years in $49 \%$ and treatment duration was shorter than 5 years in $61 \%$.

7.2.4.1. Primary symptom at onset, as reported by the patients, was tremor in $59 \%$ of patients, slowness in $22 \%$, pain in $9 \%$, handwriting disturbance in $5 \%$, stiffness in $3 \%$, dystonia in $1 \%$ and unsteadiness in $1 \%$.

7.2.4.2. Current symptoms included stiffness in $59 \%$, slowness in $82 \%$, and tremor in $94 \%$. Changes in handwriting were reported in $79 \%$, and with fine motor tasks in $73 \%$. Pain was reported by $39 \%$ of patients. $68 \%$ of patients reported difficulties in getting up from a chair, $62 \%$ with turning in bed, $68 \%$ reported shuffling or freezing, and $64 \%$ had unsteadiness with falls. Speech problems were reported by $49 \%$, and swallowing difficulties by $12 \%$. Subjective memory impairment was present in $37 \%$ of patients, $37 \%$ of patients had felt depressed at the time of the visit and $25 \%$ reported insomnia. Loss of smell on direct questioning was reported by $32 \%$, often many years before the onset of parkinsonian symptoms, and reduced facial expression had been noted by $39 \%$. $17 \%$ suffered from urinary incontinence, $27 \%$ had frequency, $21 \%$ urinary urgency, and $4 \%$ retention, $52 \%$ reported constipation, and $6 \%$ reported dizziness on getting up. 7.2.4.3. Findings: Rigidity was present in $93 \%$ and bradykinesia in all but the four patients with possible PD. Tremor at rest was seen in $82 \%$, tremor on posture in $64 \%$ and tremor on action in $29 \%$. Tremor was bilateral in $48 \%$ of patients with tremor, and also affected the legs in $48 \%$. $18 \%$ of patients with PD had dementia, as assessed by a minimental score of 24 or less, and $19.5 \%$ of those who returned the questionnaire had moderate to severe depression. $73 \%$ of patients had the akinetic-rigid subtype of PD, and $27 \%$ tremor-dominant parkinsonism.

Increasing age was associated with greater postural instability $(\mathrm{p}<0.01)$ and higher prevalence of falls $(p<0.05)$, as well as more frequent cognitive decline $(p<0.05)$ and hallucinations (both $\mathrm{p}<0.05)$. Dementia was associated with age $(\mathrm{p}<0.01)$, more severe disease $(p<0.01)$, and depression $(p<0.05)$. 
7.2.4.4. Disease severity: $26 \%$ of patients had a Hoehn and Yahr score of 3 or more (figure 3), and 34\% were dependent on others (Schwab and England score of 70 or less).

7.2.4.5. Treatment: $77 \%$ of patients had received antiparkinsonian treatment, and $70 \%$ were taking levodopa, but only $54 \%$ took an average dose of more than $300 \mathrm{mgs}$ per day. Amantadine was taken by $3 \%$, an anticholinergic by $12 \%$, a dopamine agonist by $11 \%$, and selegiline by $25 \% .79 \%$ had had an excellent or good initial response to medication, and $73 \%$ still responded with more than $50 \%$ improvent.

7.2.4.6. Side effects of medication. In the overall sample including patients who had not received levodopa, $28 \%$ had developed fluctuations in response to medication (all of whom had received levodopa), 19\% abnormal involuntary movements (all of whom had received levodopa), and $21 \%$ hallucinations (all of whom had received levodopa).

7.2.4.7. Family history and socio-economic background: A family history of parkinsonism in a first or second degree relative was reported by $16 \%$, whereby in $7 \%$ a parent was affected (both parents in one patient), in 3\% a sibling, and in $7 \%$ a second degree relative. A family history of an isolated tremor was reported by $8 \%$ of patients. $14 \%$ belonged to socio-economic group I, $21 \%$ to group $2,53 \%$ to group $3,11 \%$ to group 4 and $1 \%$ to group $5.38 \%$ of patients lived alone, $55 \%$ with their families, $3 \%$ with a friend, and $25 \%$ in care. Only $2 \%$ of patients were non-caucasian ( 3 patients with asian background).

7.2.4.8. Potential risk factors: Only $10 \%$ of patients were current smokers, but $48 \%$ had smoked at some point in the past. On average, patients had smoked for 14 (SD 17) years with an average number of 20 (SD 39.5) cigarettes per day. 58\% of patients drank alcohol with an average of 1.2 (SD 2.9) units per day. Exposure to chemicals in their life before the onset of parkinsonism was recalled by $12 \%$ of patients.

7.2.4.9. Socioeconomic consequence of the disease: $16 \%$ of patients had had to retire early, but among those aged younger than 60 years, this percentage was $33 \%$.

7.2.4.10. Factors determining disability. Schwab and England disability scores were best predicted by disease severity, as measured by Hoehn and Yahr scores ( $\mathrm{R}^{2}$ change 0.65$)$, depression scores $\left(\mathrm{R}^{2}\right.$ change 0.08$)$ and mini mental examination scores $\left(\mathrm{R}^{2}\right.$ change $0.03)$, together accounting for $75 \%$ of the variance $\left(R^{2} 0.76\right.$, adjusted $\left.R^{2} 0.75\right)$. However, Schwab and England scores not only correlated negatively with Hoehn and Yahr scores 
$(r=-0.73, p<0.0001)$ and depression scores $(r=-0.51, p<0.0001)$, and positively with mini mental examination scores $(\mathrm{r}=0.26, \mathrm{p}<0.05)$, but also correlated negatively with total UPDRS motor scores $(r=-0.3, p<0.05)$, bradykinesia $(r=-3.3, p<0.01)$ and rigidity subscores $(\mathrm{r}=-2.2, \mathrm{p}<0.05)$, but not with tremor subscores or age. In addition, patients with the akinetic-rigid subtype of $P D$, falls, fluctuations, dyskinesias (all $p<0.05$ ) and speech impairment $(\mathrm{p}<0.01)$ had significantly worse Schwab and England scores than those with the tremor-dominant subtype or without these features.

\subsubsection{Rate of progression. The median disease duration since symptom onset for} patients in each Hoehn and Yahr stage are given in table 15, separately for the overall sample and the subgroup of patients on levodopa. The rate of progression correlated positively with age at onset $(r=0.43, \mathrm{p}<0.0001)$ and age $(\mathrm{r}=0.25, \mathrm{p}<0.01)$, and negatively with dose of levodopa $(r=-0.31, p<0.001)$, and time from onset to initiation of levodopa treatment $(\mathrm{r}=-0.30, \mathrm{p}<0.005)$. Patients with dementia $(\mathrm{p}<0.0001)$, and those not receiving levodopa $(\mathrm{p}<0.002)$ had faster progression rates than those without these characteristics, whereas sex, family history, symptom at onset, response to levodopa and the presence of depression did not. Using stepwise multiple regression analysis, a number of variables that could be derived from information at onset of parkinsonism were tested for their predictive value for the subsequent rate of progression. Among the factors known at onset, only older onset age was a useful clinical predictor of fast disease progression (adjusted $\mathrm{R}^{2}=0.10$ ). When factors later in the disease course were also considered, the progression rate was best predicted by the presence of dementia, and additionally by considering whether patients were treated with levodopa, as well as their age at onset. Together these variables correctly predicted $27 \%$ of the variance of progression (table 16). No other variable contributed additionally to the prediction of rate of progression.

\subsubsection{Discussion}

\subsubsection{Clinical features}

The clinical features of the patients in this population of patients with PD in the area of London were similar to those found in the previous prevalence studies in the UK (Aberdeen (Mutch, 1986b) and Northamptonshire (Sutcliffe, 1985)), suggesting no differences between these populations which differed in time and location. However, the 
population reported from Aberdeen were overall older, had a longer disease duration, and greater disability. This difference is likely to be related to different ascertainment methods, as in this study not only all patients who had a diagnosis of PD were examined, but also those with an isolated tremor with onset after age 50, many of whom were found to have early PD (7\% of all patients with PD). In addition, the population of London is relatively young, which is reflected in the younger age of this sample. This may additionally explain the greater disease severity in the Aberdeen study, as postural instability, which is an important features in the classification of the Hoehn and Yahr scale, increases significantly with increasing age in the general population (Waite, 1996).

\subsubsection{Treatment}

It is noteworthy that only $77 \%$ of patients had ever received antiparkinsonian treatment, and $46 \%$ received doses without significant benefit. This may reflect cautious prescription of antiparkinsonian drugs in the elderly, where it is more likely to induce cognitive disturbance and hallucinations, or conscious delay of 1-dopa therapy to avoid future complications. Another explanation is however, that some features of PD such as postural instability and slowness are accepted as symptoms of old age. Thus, if antiparkinsonian medication treatment had been given to more patients and in higher doses, a greater number of patients may have responded and their disability and quality of life improved, but they may also have experienced more adverse events, such as dyskinesias, fluctuations, hallucinations and orthostatic hypotension.

\subsubsection{Complications of disease and treatment}

In comparison to clinic-based studies (Senard, 1997; Chrischilles, 1998), this study found a low rate of symptomatic orthostatic hypotension (6\%). Complications of longterm treatment with antiparkinsonian medication such as fluctuations in motor response and dyskinesias were also lower than reported in clinic-based studies (Chrischilles, 1998; Cedarbaum, 1991; Miyawaki, 1997) and present in less than a third of all patients. On the other hand, psychiatric side effects of medication, dementia and depression were each seen in approximately $20 \%$ of patients, which is similar to the rates reported in the literature, although comparisons are difficult to make due to varying methodology 
(Brown and Marsden, 1984;Mayeux, 1988). Postural instability with falls, however, had clearly occurred in the great majority of patients (64\%). The most likely explanation for the difference of the findings of this study with previously reported rates of complications of advanced disease and treatement is the high average age of this population (72 years). Dyskinesias and motor fluctuations occur less frequently in patients with older than those with young onset of PD (Diamond, 1989; Kostic, 1991), whereas postural instability with falls, dementia, and hallucinations are known to increase with increasing age in PD, and are rare in patients below the age of 60 (Waite, 1996; Quinn, 1987). An additional explanation is the lower mean dose of levodopa in this sample than in clinic-based samples, which is likely to result in a higher disease severity but lower rate of treatment related complications.

\subsubsection{Disability}

More than $30 \%$ of patients reported being dependent on others at least to some degree. A substantial proportion of patients, particularly among those younger than 60 had had to retire early due to PD. The rates of early retirement are higher than reported by Mutch et al (Mutch, 1986b), but are once again likely to be due to the younger average age in this sample. Disability, as measured by the Schwab and England scale, was mainly related to disease severity, as might be expected, but also to the presence of depression and dementia, but not to tremor scores, or to fluctuations and dyskinesias, which did not further explain the variance of progression rates. The lack of impact of motor complications on disability in this sample may be a result of the low rate and mild severity of these complications due to low doses of levodopa, but may also be related to the relatively old mean age. It is thus likely to be different in patient samples with a younger population on high doses of treatment, many of whom warrant stereotactic surgery to alleviate the severe motor fluctuations and dyskinesias (Schrag, 1998).

\subsubsection{Family history}

A family history of parkinsonism was reported by $17 \%$ of patients with $\mathrm{PD}$, which is similar to what has been reported in clinic-based and population-based samples of patients with PD (Payami, 1994; Sutcliffe, 1992) and higher than in control samples. 
(Payami, 1994), supporting the role of genetic factors in a proportion of patients with PD.

\subsubsection{Rate of progression}

The rate of progression between patients can vary greatly (Poewe, 1998). In keeping with this this study found a wide range of disease durations in patients with similar Hoehn and Yahr stages. The median disease duration for each stage in this sample was similar to those reported by Hoehn and Yahr (Hoehn and Yahr, 1967), and by Marttila and Rinne (Marttila, 1977; mean disease duration was reported). However, patients in Hoehn and Yahr stage V in this study had a shorter disease duration than those in stage IV, presumably due to the small number of patients and the high rate of dementia in this group. Patients receiving levodopa had longer disease durations at all Hoehn and Yahr stages (apart from stage V), indicating a similar rate of progression with and without levodopa, but a longer time patients spend in each stage, probably due to the symptomatic effect of levodopa.

\subsubsection{Factors influencing disease progression}

Among all variables known at onset, older age at onset was the best predictor of a relatively fast disease progression. Although only a small percentage of the variability of the rate of progression could be explained with this factor, this confirms findings by other authors who reported a better prognosis for younger patients (Goetz, 1988; Blin, 1989; Diamond, 1989). Unlike other studies (Marttila, 1977; Roos, 1996; Zetusky, 1985), symptom at onset was not a useful clinical predictor of the rate of progression in this study. When factors later in the disease course were also considered, the presence of dementia predicted faster progression of motor symptoms best, as has been reported previously by Biggins et al (Biggins, 1992). Treatment with levodopa, on the other hand, resulted in an apparent slowing of progression, which was probably achieved through its symptomatic effect. However, levodopa was not withdrawn and the underlying severity of parkinsonism was therefore not assessed. Despite this confounding factor, patients who had received levodopa therapy later were also found to have a slower disease progression. This could be explained by a beneficial effect of delaying levodopa therapy 
on disease progression, but is more likely to be due to a slower disease progression in these patients, which necessitated initiation of levodopa later.

The results of this study on the factors influencing rate of progression should be interpreted cautiously as information on events before the study were collected retrospectively, mainly from the patients and carers. However, the features and complications of patients with PD in this community-based sample are likely to be representative of the overall population of patients with $\mathrm{PD}$, and can provide the basis for provision of health care resources as well as an estimation of the rate of progression in an unselected sample of patients with PD. 


\subsection{Dyskinesias and motor fluctuations in Parkinson's disease}

\subsubsection{Background}

The occurrence of dyskinesias and motor fluctuation on chronic levodopa treatment is a major problem in the long-term management of patients with PD. It has been estimated that after initiation of treatment with levodopa approximately $10 \%$ of patients per year develop motor fluctuations (Marsden, 1977), and in clinic-based studies about 50\% suffer these complications after 5 years of treatment (Dupont, 1996; Sweet, 1975). However, considerable controversy exists on whether their occurrence is primarily related to the disease itself or to its treatment, and whether, as a consequence, treatment with levodopa should be delayed or kept at a low dose (Fahn, 1984; Markham, 1986; Melamed 1986; Poewe, 1986; Lees, 1981; Roos, 1990; Blin, 1988; Cedarbaum, 1991). In animal studies the occurrence of motor complications is related to the degree of loss of dopaminergic neurons in the substantia nigra (Boyce, 1990a; Schneider, 1989), and patients with MPTP-induced parkinsonism, who have severe depletion of nigral dopaminergic neurons (but are also young), develop motor complications rapidly and severely (Ballard, 1985). However, the rate of motor complications in MPTP-treated animals also depends on the duration and dose of treatment with levodopa (Boyce, 1990b; Arai, 1996). In clinic-based samples of patients with PD, motor complications have been shown to occur more frequently and earlier in patients with younger onset (Kostic, 1991), and to increase with longer disease duration and greater disease severity (Lesser, 1979; Tanner, 1985a; Parkinson Study Group, 1996). It has also been claimed that dyskinesias occur more frequently in women if disease duration is longer than 5 years (Lyons, 1998; Parkinson Study Group, 1996). On the other hand, higher doses of levodopa (Lees, 1981; Tanner, 1985a; Barbeau, 1980), and longer duration of treatment (Miyawaki, 1997) have been found to be associated with higher rates of these complications, and initiation of treatment with other antiparkinsonian drugs has resulted in lower rates of or delayed motor fluctuations and dyskinesias (Lees, 1981; Brannan, 1995; Rinne, 1987). However, many of these variables are related, such as age of onset, disease duration, duration of treatment, disease severity and dose of levodopa. It is therefore difficult to determine which of these factors are most important for the development of motor complications. In addition, clinic-based samples are 
probably not representative of the overall population of patients with PD. They may be biased towards more severely affected or younger patients, or those with a higher rate of complications. Patients seen in specialist neurological clinics are also more likely to be treated with high doses and more complex regimens of medication.

I therefore assessed the prevalence of motor fluctuations and dyskinesias in this unselected population-based sample of patients with $\mathrm{PD}$, and investigated the influence of disease- and treatment-related variables on their occurrence.

\subsubsection{Methods}

All 124 patients with probable PD were included. Patients with atypical parkinsonism, vascular parkinsonism, drug-induced parkinsonism, and those with parkinsonism following dementia were excluded. During the diagnostic visit, patients were questioned about the presence of oscillations in motor response (predictable wearingoff, unpredictable on-off fluctuations and sudden off-periods, as defined in the Unified Parkinson's Disease Rating Scale (UPDRS; Fahn, 1987) and involuntary movements (peak-dose, diphasic and off-period) related to treatment, and their time of first occurrence. Six patients in whom dyskinesias were present on examination, but not noticed by the patient or carer, were included in the group of patients with dyskinesias.

\subsubsection{Statistical analysis.}

The main analysis for this part of the study was performed on the subgroup of patients who had received levodopa, and consisted mainly of calculating rates and proportions. A statistical level of 5\% was considered as significant in most analyses. However, in order to take into account multiple comparisons between patients with and without dyskinesias, a statistical threshold of $\mathrm{p}<0.005$ was considered to be sufficiently conservative for this analysis. Differences with $p<0.01$ and $p<0.05$ were considered as marginally significant. In order to determine which factors contribute most to the prediction of the occurrence of motor fluctuations and dyskinesias, stepwise discriminant analysis was performed entering all variables that had differed between those with and those without complications at the $5 \%$ level $(\mathrm{p}<0.05)$. 


\subsubsection{Results}

\subsubsection{Prevalence of motor fluctuations and dyskinesias}

The characteristics of all patients on levodopa are given in table 17. In the overall sample of patients with PD, 35 (28.7\%) had experienced motor fluctuations and 24 (19.4\%) dyskinesias. Apart from one patient in whom levodopa had been discontinued due to psychiatric side effects, all of these patients were currently taking a levodopa preparation. Among the patients who had received levodopa $(n=87,70 \%)$, the rate of motor fluctuations was $41.2 \%$ and that of dyskinesias $27.9 \%$. If only the patients who had an excellent or good response to levodopa ( $>50 \%$ improvement) were considered $(\mathrm{n}=38,31 \%)$, the rates were $48.6 \%$ and $27.8 \%$, respectively. Among patients receiving doses of more than 300 mgs levodopa per day $(n=44,36 \%$; average dose 423.3 , SD 227.6), the rates were 61.4 and $40.0 \%$, respectively.

\subsubsection{Rate of motor complications by disease duration, treatment duration and} Hoehn and Yahr stage

The rates of occurrence of motor fluctuations and dyskinesias in levodopa-treated patients with a disease duration of less than 5 years were 10.5 and $5.0 \%$, respectively. After a disease duration of 5-10 years they had occurred in 40 and 15\%, respectively, and after more than 10 years of disease 66.7 and $58.3 \%$. These rates were lower if the overall sample including patients not on levodopa was considered, and greater when only those who had a good or excellent response to levodopa, or those with levodopa doses of more than 300 mgs were considered (table 18).

After a treatment duration of less than 5 years, 27.3 and $18.2 \%$ of patients on levodopa had developed fluctuations and dyskinesias, respectively, after 5 to 10 years of treatment 53.8 and $29.6 \%$, and after more than 10 years all patients had developed dyskinesias and/or motor fluctuations.

Motor complications had not occurred in patients on levodopa with a Hoehn and Yahr stage of 1 or 1.5 (as assessed during the diagnostic visit), but $42 \%$ of those in stages 2 or 2.5 had motor fluctuations and $28.9 \%$ had dyskinesias. In stage 3 patients, motor fluctuations were present in 50\% and dyskinesias in $42.9 \%$, and in patients in Hoehn and Yahr stages 4 or 5 they were present in 71.4 and $60 \%$, respectively. 


\subsubsection{Subtypes of motor fluctuations and dyskinesias}

Motor fluctuations were predominantly 'wearing off'-fluctuations ( $n=30,34 \%$ of patients on levodopa), but $9(10 \%)$ patients had more severe on-off fluctuations, and 6 patients $(7 \%)$ experienced sudden off periods. Dyskinesias were predominantly peak dose $(n=15,17 \%)$, but 5 patients $(6 \%)$ had diphasic dyskinesias and $9(10 \%)$ off-period dystonia.

Wearing-off had developed in $20.5 \%$ of those treated for less than 5 years, $44.4 \%$ of those treated for 5 to 10 years, and $100 \%$ of those patients treated for more than 10 years, whereas unpredictable on-off fluctuations had not developed in patients with a treatment duration of less than 5 years, and were present in $18.5 \%$ of those with a treatment duration of 5 to 10 years, and $50 \%$ of those with a treatment duration of more than 10 years. Sudden off periods had occurred in $2.3,7.4$ and $37.5 \%$ of patients after treatment durations of $<5,5$ to 10 , and more than 10 years, respectively. Peak-dose dyskinesias were present in $11.4 \%$ of patients with a treatment duration of less than 5 years, $29.6 \%$ with a treatment duration of 5 to 10 years, and $87.5 \%$ of patients with a treatment duration of more than 10 years. Off-period dystonia was reported by $2.3,14.8$ and $50 \%$, respectively, and diphasic dyskinesias by no patients with a disease duration of less than 5 years, $3.7 \%$ of those with a disease duration of 5 to 10 years and $50 \%$ of those with a disease duration of more than 10 years, respectively. Patients with diphasic dyskinesias and off period dystonia as well as wearing-off fluctuations had a younger age at onset $($ all $\mathrm{p}<0.05)$.

\subsubsection{Time to onset of complications}

The mean time from symptom onset to development of fluctuations, as reported by the patients, was 6.5 (SD 4.1) years, and to development of dyskinesias 6.7 (SD 3.1) years. The mean time from start of levodopa to the onset of fluctuations and dyskinesias was 4.8 (SD 3.4) and 5.7 (SD 2.7) years, respectively.

The latency from onset of symptoms to development of fluctuations and dyskinesias correlated positively with the time elapsed between disease onset and initiation of treatment with levodopa $(\mathrm{r}=0.59, \mathrm{p}<0.05$ and $\mathrm{r}=0.54, \mathrm{p}<0.05$, respectively). This could be due to a faster development of motor complications after levodopa was given earlier, but also to more rapidly progressive disease warranting earlier introduction of levodopa 
in some patients. The patients with more rapidly progressive disease would then be expected to be more severely disabled after the same duration of disease. Thus, disease severity should be negatively correlated with time from onset to initiation of levodopa, when controlled for disease duration. However, there was no such correlation $(r=0.15$, $\mathrm{ns}$ ), indicating that more rapidly progressive disease is not the explanation for the earlier development of motor complications in patients treated sooner with levodopa. Time from onset to development of complications was also positively associated with age $(\mathrm{r}=0.30$, ns for fluctuations, and $\mathrm{r}=0.76, \mathrm{p}<005$ for dyskinesias), indicating that older patients develop motor complications later. However, they also received lower doses of levodopa $(r=-0.30, p<0.005)$. Thus, when adjusted for dose of levodopa, age no longer correlated with time to onset of motor complications. Age at disease onset also correlated inversely with time to onset of fluctuations $(r=-0.64, \mathrm{~ns})$, and weakly inversely with time to onset of dyskinesias $(\mathrm{r}=-0.24, \mathrm{~ns})$. Thus, patients with younger onset tended to have motor complications earlier than those with older onset.

\subsubsection{Differences between patients with and without motor fluctuations and dyskinesias}

Patients with motor fluctuations had significantly longer disease duration, treatment duration, higher doses of levodopa, and marginally longer time since diagnosis than those without. They also had greater disability, as measured by the Schwab and England scale, and marginally greater disease severity, as measured by the Hoehn and Yahr scale, more often had a good response to levodopa, and were receiving a levodopa dose higher than $300 \mathrm{mgs}$ per day. They also tended more often to have a family history of parkinsonism in a first or second degree relative and the akinetic-rigid subtype of parkinsonism (table 19).

Patients with dyskinesias had a significantly longer treatment duration and were more severely disabled than those without. They tended to have longer disease duration, greater disease severity, and to have received a higher levodopa dose, and more often reported falls than those without dyskinesias (table 19). Men and women did not differ significantly with respect to the occurrence of motor fluctuations or dyskinesias, even when only those with a treatment duration of more than 5 years were considered. Age, symptom at onset, or time from onset to initiation of treatment, did not differ between 
the groups.

\subsubsection{Discriminant analysis}

In order to understand the influence of different variables on the occurrence of motor complications, two stepwise discriminant analyses were performed, separately for dyskinesias and motor fluctuations as the target variables.

Disease duration correctly predicted the occurrence of motor fluctuations in $72.0 \%$. The only variable that increased the prediction of occurrence of fluctuations further was dose of levodopa, which increased the percentage of correctly categorised cases to $74.8 \%$ (sensitivity 57.6 , specificity 86.0 , positive predictive value $73.0 \%$, negative predictive value $75.4 \%$ ). No other disease- or treatment-related factor helped predict the occurrence of fluctuations significantly once disease duration and dose of levodopa were accounted for.

Dyskinesias were predicted by duration of treatment with levodopa alone in $69.1 \%$ (sensitivity 45 , specificity 55 , positive predictive value $66.7 \%$, negative predictive value $83.6 \%$ ), whereas disease duration, time since diagnosis, disease severity, or levodopa dose did not help further in predicting their occurrence.

\subsubsection{Patients without motor complications after more than 10 years of treatment} with levodopa

A subgroup of 5 patients had not developed motor fluctuations even after a disease duration of 10 years, and 5 patients had not developed dyskinesias. Patients without fluctuations after more than 10 years disease duration had a shorter disease duration than those with fluctuations $(\mathrm{p}<0.05)$. Those without dyskinesias had a lower dose of levodopa $(\mathrm{p}<0.05)$. Age, age at onset, treatment duration, Hoehn and Yahr stage, Schwab and England score, and sex were not significantly different between these patients and those who had developed motor complications.

\subsubsection{Quality of life}

Quality of life was not significantly different between patients with and without motor fluctuations and dyskinesias. However, when quality of life of patients was evaluated according to response to treatment (which was associated with higher doses of 
levodopa), it was significantly better in those with a good response to levodopa than in those with a moderate or poor response in those with a disease duration of less than 5 or more than 10 years (both $\mathrm{p}<0.05$ ).

\subsubsection{Discussion}

The rate of both fluctuations and dyskinesias increased with increasing disease and treatment duration as well as advancing disease severity. Thus, no patient in Hoehn and Yahr stage I or I.5 had any motor complications, whereas more than $80 \%$ of those in stages 4 or 5 had motor fluctuations and more than $50 \%$ dyskinesias. Similarly, after a disease duration of more than 10 years, more than $60 \%$ had motor fluctuations, and more than $50 \%$ dyskinesias.

These rates of motor complications are lower than rates of response fluctuations and dyskinesias in patients after the same disease and treatment durations in large clinicbased, cross-sectional studies (Cedarbaum, 1991; Miyawaki, 1997; Fahn, 1992). Patients in these studies, which may be biased towards younger subjects or those with more severe disease, also used higher mean doses of levodopa. To my knowledge, no comparable data are available on the rate of dyskinesias in an unselected population of patients with $\mathrm{PD}$, and only one study has previously provided population-based data on the occurrence of motor fluctuations (Tandberg, 1995). In that study, which also included patients not receiving levodopa, the rate of motor fluctuations was $21 \%$, and $28 \%$ in those classified as 'definite PD', who were required to have a good response to levodopa. Although this rate is similar to the rate of $29 \%$ in the overall group of patients with $\mathrm{PD}$ in this study, it is considerably lower than the rate in the subgroup of patients receiving levodopa in this study, despite a higher mean dose of levodopa and longer mean disease and treatment duration in this study. Although there is no obvious explanation for this, it may be due to methodological differences in case ascertainment, diagnostic classification and a different definition of fluctuations, which was not specified further in the cited paper. In this study, great care was taken to include even patients who had noticed only mild wearing-off in the group with motor fluctuations. It is generally believed that dyskinesias and motor fluctuations develop in about $50 \%$ of patients on treatment with levodopa after 5 years (Lang, 1998), whereas the relationship of the occurrence of dyskinesias to duration of levodopa treatment is less 
clear (Fahn, 1984). However, the rates of these complications on antiparkinsonian treatment reported in the literature vary greatly, ranging form 19 to $80 \%$ of patients experiencing fluctuations after 5 or 6 years of treatment with levodopa (Poewe, 1986; Brannan, 1995), and from 0 to $66 \%$ developing dyskinesias during the first year of treatment (Barbeau, 1980; Brannan, 1995). A prospective study comparing standard release and controlled release levodopa/carbidopa (Block, 1997) found fluctuations or dyskinesias in only $20 \%$ (patient-rated) or $16 \%$ (observer-rated) of patients after 5 years in either treatment arm, whereas another study comparing standard and sustained release levodopa/benserazide (Dupont, 1996) found dyskinesias in approximately $40 \%$ and fluctuations in approximately $60 \%$ after 5 years of treatment. The differences between these studies suggest that certain variables can influence the occurrence of motor fluctuations and dyskinesias on levodopa treatment. They have partly been attributed to different dosages of levodopa and the initial lack of use of peripheral decarboxylase inhibitor (Poewe, 1986; Lees, 1981; Barbeau, 1980; Block, 1997), as well as the prior or concomitant use of other medication, such as dopamine agonists (Brannan, 1995; Rinne, 1987; Hely, 1994; Montastruc, 1994). Age at onset, age, sex, time from onset to initiation of levodopa and severity of disease may also influence the occurrence of dyskinesias (Blin, 1988; Kostic, 1991; Parkinson Study Group, 1996; Lyons, 1998; de Jong, 1987; Pederzoli, 1983). In the present study duration of disease and treatment as well as dose of levodopa were associated with a higher occurrence of motor complications, whereas age, sex, age or symptom at onset were not different between those with and those without fluctuations or dyskinesias. A positive family history of parkinsonism was also weakly associated with the occurrence of motor complications. Although no other features were different between those with and without a family history of parkinsonism, this may indicate that a subgroup of patients with genetically determined parkinsonism has a higher propensity to develop these complications.

When the interrelations of these disease- or treatment-related variables were accounted for by using stepwise discriminant analysis, and all variables that differed between patients with and without motor complications were entered, disease duration was the best predictor for the occurrence of motor fluctuations, although dose of levodopa helped predict a further $2 \%$ of cases. Dyskinesias, on the other hand, were best 
predicted by treatment duration alone. Age or symptom at onset, current age, sex or family history did not contribute further to the prediction of motor fluctuations or dyskinesias. However, the sensitivity of the prediction of dyskinesias was less than $50 \%$, indicating that other variables, not identified in this study, have additional influence of the occurrence of dyskinesias. The importance of treatment-related factors for the development of motor complications is supported by a number of further findings: 1. A shorter time from onset to treatment with levodopa was associated with earlier motor complications, which could not be adequately explained by more rapid disease progression. 2. The rate of motor fluctuations and dyskinesias was lower than that reported in clinic-based samples that employed higher doses of levodopa after a similar disease duration (Dupont, 1996; Miyawaki, 1997). 3. The rate of motor complications was higher in the subgroup of patients on levodopa than in the overall sample of patients with PD with the same disease duration (since no patient who had not received levodopa experienced these complications), and even greater in those patients who a good response to levodopa or received more than $300 \mathrm{mgs}$ levodopa per day despite similar disease severity. 4 . The difference in treatment duration between patients with and without dyskinesias was greater than the difference in disease duration. 5. Patients who had not developed dyskinesias after more than 10 years differed from those who had only in receiving lower doses of levodopa. All these findings indicate that, although disease duration is an important factor in the development of motor complications, earlier and longer treatment with, as well as higher doses of, levodopa are associated with a higher risk of their occurrence. The results of this study are also similar to those in a recent, clinic-based study of patients with $\mathrm{PD}$, in which longer treatment duration and shorter latency to initiation of levodopa treatment were identified as the main risk factors for motor fluctuations and dyskinesias (Denny, 1999).

However, it should also be noted that a large percentage (42 and 33\%) of patients on levodopa without dyskinesias and fluctuations were not receiving doses sufficient to provide satisfactory benefit, and those with unsatisfactory response to medication in the initial years of disease as well as in later stages after more than 10 years of disease duration had significantly worse quality of life than those with a good response to medication. Thus, although not experiencing motor complications, which were mild in 
the great majority of patients, these patients were failing to derive adequate benefit from medication, which resulted in poorer quality of life.

I believe that these results can be generalized to the overall population of patients with $\mathrm{PD}$, as an unselected sample was evaluated, and as high diagnostic accuracy was the primary aim of the study. However, as this was a cross-sectional assessment rather than a longitudinal follow-up study, there are two main limitations to this study: Firstly, the drug treatment of patients was not adjusted in a uniform way, as they were under the care of many different clinicians, and, secondly, most of the retrospective information was reported by the patients themselves or derived from the records of their general practitioners. In particular, patients were not examined before the start of treatment, and the time of onset of complications was reported by the patients themselves and their carers. However, the patients' own report of onset of symptoms is likely to be more accurate than the date of diagnosis or the first recording of motor complications, and the results were unchanged, but less significant, when date of diagnosis was used instead of duration of disease.

In summary, response fluctuations and dyskinesias had developed in approximately 40 and $30 \%$ of patients with $\mathrm{PD}$, respectively. These rates increased with disease duration and severity, but they were more strongly related to treatment-related factors, such as duration and dose of levodopa. Earlier treatment with levodopa was associated with earlier development of motor complications. However, less effective treatment and poorer quality of life associated with inadequate dosage of levodopa may be the price to pay for a low rate of motor complications in patients with PD. 


\subsection{Depression in Parkinsons's disease}

\subsubsection{Background}

Depression occurs more frequently in patients with $\mathrm{PD}$ than in the age-matched population (Warburton, 1967; Robins, 1976; Cantello, 1986; Ehmann, 1990; Gotham, 1986). It is presumed that in many cases it is related to the neurochemical changes in PD (Charlton, 1997; Mayeux, 1984; Mayeux 1986; Ring 1994), but its mechanism is poorly understood and it is unclear why some patients develop depression and others do not. There is no clear relation to severity or duration of disease or to the occurrence of specific parkinsonian symptoms (Brown, 1995). In addition, depression does not improve consistently with medical or surgical antiparkinsonian treatment, except for depression as an off-period symptom (Lieberman, 1998; Quinn, 1998; Fetoni, 1999). It has been suggested that other personal and social factors (MacCarthy, 1989), and reaction to the disease and its consequences play a role. However, disability and personal and social factors still only account for less than $50 \%$ of the variance of depression (MacCarthy, 1989).

The World Health Organisation (WHO, 1980) has suggested a useful model to assess the impact of chronic disease on patients' lives: The objective impairment caused by the disease, such as presence and severity of symptoms, may lead to disability, the functional deficit resulting from impairment. Impairment and disability can both result in handicap, which depends on the patient's social and personal circumstances and his/her social roles and physical and emotional make-up (WHO, 1980). Although handicap usually results from the disability associated with impairment, it can result directly from impairment even without any accompanying disability (e.g. symptoms causing social embarrassment). Furthermore, patients with the same objective impairment (symptom severity) may experience very different degrees of disability and handicap. Mediating factors like personality traits or social circumstances can prevent or increase handicap in patients with the same level of disability or impairment. In order to improve our understanding of the mechanisms of depression in $\mathrm{PD}$, some of the factors that may contribute to depression in this population-based sample of patients with PD were investigated. Taking the WHO model into account the relation of depression to various motor and non-motor symptoms of $\mathrm{PD}$, and to the resulting 
disability and self-reported handicap as indexed by quality of life (QoL) measures was evaluated.

\subsubsection{Methods}

All 124 patients diagnosed with probable PD were included in this part of the study. On the BDI, higher scores (range 0-63) indicate more severe depression, and the cut-off score for moderate to severe depression used was 17 (18 or more indicates moderate to severe depression; Beck, 1961).

\subsubsection{Statistical analysis}

Clinical and historical information on features and complications of PD were analysed with regard to their influence on depression scores. In addition, the association between depression scores and the results of the QoL questionnaire was investigated. Akinesia scores were calculated as the sum of items 19 and 23 to 26 of the UPDRS for both sides, and the tremor score from items 20 and 21 for both sides. The tremor-dominant subtype of PD was defined as patients with a ratio of tremor to bradykinesia score (rigidity and postural instability subscores from the UPDRS motor scale and history) $\geq 0.5$, and the akinetic-rigid subtype as patients with a ratio of $<0.5$. The subdimensions and summary scores of the QoL instrument were calculated according to the respective scoring algorithms. Mean values were compared by the Mann-Whitney test, and Spearman rank correlations were calculated for examining associations of variables. Categorical data were analysed using the Chi-square and Fisher's excact tests, where appropriate. Significance was accepted at the $1 \%$ level $(\mathrm{p}<0.01)$. Stepwise multiple regression analysis was used to determine the factors contributing independently to depression scores at a significance level of $\mathrm{p}<0.05$.

\subsubsection{Results}

The response rate was $78 \%$.

\subsubsection{Extent of depression}

The mean BDI score in this sample was 12 (SD 10.5; ranging from 0 to 54 ). 35 (36.1\%) of the responders had mild depression (BDI score 9-16), 10 (10.3\%) moderate (BDI 
score 17-23), and $9(9.3 \%$ ) severe depression (BDI score $>24)$. Thus, moderate to severe depression was reported by $19.6 \%$ of the sample.

\subsubsection{Relation of depression to patient-and disease characteristics}

There was a moderate, but significant correlation of increasing depression scores with higher disease severity as measured by the Hoehn and Yahr scale $(r=0.36, p<0.001)$ and higher akinesia scores $(r=0.37, r<0.01)$, and a trend for a correlation with higher tremor scores $(r=0.25, \mathrm{p}<0.05)$, but not with age, age at onset or disease duration, even after controlling for disease severity. As shown in table 20, depression scores were significantly higher for patients who reported recent deterioration of their health $(p<0.001)$ and those with falls $(p<0.01)$ than for those without these features. There was also a trend for patients with the akinetic-rigid subtype of $\operatorname{PD}(\mathrm{p}<0.05)$, with postural instability $(p<0.05)$, a mini mental score of less than $25(p<0.05)$, self-reported hallucinations $(p<0.05)$, stiffness $(p<0.05)$ and impairment of dexterity $(p<0.05)$ or speech $(\mathrm{p}<0.05)$, to have higher depression scores. Gender, onset before or after age 55 , current age above or below 60 , or the presence or absence of tremor or rigidity, and self-report of dyskinesias, on-off fluctuations or of response to levodopa did not have a significant effect on depression scores.

A proportion of patients with moderate to severe depression were in the early stages of illness. However, overall, more than $90 \%$ of patients with depression scores of 18 or more had Hoehn and Yahr stages of 3, 4 or 5 (see figure 4). However, patients with BDI scores of 18 or more had significantly greater postural instability, as measured on the UPDRS motor scale ( $\mathrm{p}<0.001$ ), more frequently reported recent deterioration of their health $(p<0.005)$, and tended to have lower MMSE scores $(p<0.01$; table 21$)$. No significant difference was seen between depressed and non-depressed patients with regard to age, sex or age at onset, tremor, rigidity and akinesia scores, presence/absence of fluctuations, dyskinesias or hallucinations, socioeconomic status or living conditions. 


\subsubsection{Relation of depression to disability}

Depression scores increased with decreasing Schwab and England scores, indicating greater depression with increased disability, whether assessed by the examiner ( $\mathrm{r}=-$ $0.57, \mathrm{p}<0.0001)$, or by the patient $(\mathrm{r}=-0.60, \mathrm{p}<0.0001)$. There was a strong correlation between the observer's and the patient's rating of disability on the Schwab and England scale $(\mathrm{r}=0.89, \mathrm{p}<0.0001)$. Patients with BDI scores of 18 or more had significantly greater disability scores as rated by the examiner $(p=0.002)$ or the patient $(p=0.0001$; table 21).

\subsubsection{Relation of depression to self-rated health status}

Depression scores were most closely related to subjectively perceived impairment of cognition, as measured on the PDQ-39, $(r=0.67, \mathrm{p}<0.0001)$. They were also significantly correlated with impairment of all other aspects of QoL, although less strongly with impairment of social support, as measured on the PDQ-39 ( $r=0.32$, $\mathrm{p}<0.01$ ). The patients with a BDI score of 18 or more had significantly worse scores on all areas of QoL (see table 21).

\subsubsection{Multiple regression analysis}

In order to determine the factors that contributed most to depression, a stepwise multiple regression analysis entering all factors that had shown correlation with depression scores or resulted in significantly different depression scores was performed. First, parameters measuring impairment (Hoehn and Yahr stage, akinesia score, postural instability, MMSE score and recent deterioration) were entered. Second, disability as measured by the Schwab and England scale, by the assessor and by the patient, were entered in a separate analysis. Third, a stepwise multiple regression was performed with QoL scores as the independent variables to determine which specific aspects of handicap most closely related to depression. Finally, an overall analysis was carried out, using measures of impairment, disability and handicap in a single regression analysis, to determine the best overall predictors of depression (see table 22). 


\subsection{Disease-characteristics - Degree of impairment}

Disease severity, as measured on the Hoehn and Yahr scale, accounted for $19 \%$ of variance in BDI scores, and the self-report of recent deterioration for an additional $11 \%$. Together they explained $28 \%$ of the variance of depression scores. No other disease-related factor or patient characteristic additionally contributed to the prediction of depression scores (table 22).

\subsection{Disability}

Disability, as measured by the observer-rated Schwab and England scores, was the strongest objective predictor of depression and explained $34 \%$ of the variance. Patientrated Schwab and England scores alone explained 51\% of the variance (table 22).

\subsection{Quality of life}

Stepwise multiple regression with QoL subscores revealed that, from the patient's perspective, impairment in the areas of cognition, emotional well-being, stigma and activities of daily living (as measured on the PDQ-39) predicted depression scores in $66 \%$ of patients (not shown). When the domain that measures depression (emotional well-being) was excluded from the analysis (as it measures the same content as the BDI), subjective impairment of cognition and the feeling of stigmatisation still predicted $53 \%$ of the variance of depression scores (see table 22).

\subsection{Overall: impairment, disability and $Q o L$}

When observer- or patient-rated disability, as measured by the Schwab and England scale, and measures of impairment were added to the model, only observer-rated disability and the report of recent deterioration contributed additionally to the prediction of depression scores. Overall, $65 \%$, or, if the domain of emotional functioning is excluded, $60 \%$ of the variance of depression scores could be explained taking these variables into account.

\subsubsection{Discussion}

\subsubsection{Prevalence of depression in $P D$}

Moderate to severe depression with BDI scores of more than 17 was seen in $19.5 \%$ of patients with PD. Studies in clinic-based samples or using different instruments have reported a wide range of depression rates (Celesia, 1972; Cummings, 1992; Mayeux, 1981), but in the only comparable, population-based study in Sweden a similar rate of 
$24 \%$ was found when using the same instrument and cut-off score, although the authors diagnosed major depression in only $7.7 \%$ of patients in that sample (Tandberg, 1996). The BDI was considered a suitable instrument to measure the severity of depression in patients with $\mathrm{PD}$ in this study and to examine the influence of determining factors, as a) it is widely used and has been repeatedly shown to be valid in patients with PD, b) it enables quantitative assessment of the severity of depression and c) its validity and internal consistency have been shown to be comparable in patients with PD and controls (Levin, 1988).

\subsubsection{Factors contributing to depression}

Greater disease severity was overall associated with higher depression scores, but the prevalence of depression had a bimodal distribution with a percentage of patients being depressed at initial stages of the disease (Hoehn and Yahr stage 1.5), and a larger percent at later stages (4 and 5). This finding has also been previously reported (Celesia, 1972; Starkstein, 1990), and may indicate reactive depression soon after diagnosis, improvement of depression in the process of adaptation to a slowly advancing disease and improvement in parkinsonian features after initial treatment, and subsequent recurrence of depression as the disease and its response to medication deteriorates.

In accordance with a previous report by Starkstein et al (Starkstein, 1998), subgroups of patients with the akinetic-rigid subtype of parkinsonism and those with cognitive impairment (Starkstein, 1990) were more likely to be depressed than those without these characteristics. In addition, postural instability and recent deterioration were also found to be associated with higher depression scores, whereas age and type of onset, sex, age and the presence of fluctuations or dyskinesias did not influence patients' overall mood.

However, measures of impairment could predict depression in only a minority of patients. Objective disease severity alone predicted depression scores in less than $20 \%$, which is similar to the findings of previous studies (Brown, 1995). The consistent finding that only a small proportion of depression scores can be predicted by disease severity suggest that a separate mechanism underlies the development of mood disorder than that of motor dysfunction in patients with PD. 
Although no other disease-related factors contributed to prediction of depression scores, the patients' own report of a recent deterioration further increased the prediction of depression scores. Disease progression was not assessed objectively in this crosssectional study, and a subjective report of deterioration may be influenced by the depressed patient's own pessimistic view of his condition. However, this finding is in agreement with previous studies which found that deterioration is an important factor in developing depression in PD (Starkstein, 1998; Brown RG, 1989), and studies in other chronic diseases, where a progressive course is associated with higher depression scores (Starkstein, 1992; McIvor, 1984; Mindham, 1981; Moos, 1964).

Disability, on the other hand, correlated strongly with depression and was the strongest objective predictor of depression in PD in this study. It explained $34 \%$ of the variance of the depression scores, which is more than twice that found in previous studies (Ehmann, 1990; Gotham, 1986; MacCarthy, 1989). As may be expected, the patients' own rating of disability correlated more strongly with their depression scores. On the other hand, objective measures of impairment, such as severity of motor symptoms or disease severity, did not contribute further to depression scores.

Although disability in this study was the single most influential predictor of depression, the degree of depression was best predicted by considering the patients' own judgement of their handicap, which explained more than $50 \%$ of the variation. It may be argued that self-report of poor QoL in depressed patients may reflect their pessimistic outlook on the future, and thus depression conversely influence self-rated QoL scores. However, the uneven distribution of correlations, with subjective impairment of mobility and physical functioning least impaired, suggests that this is not the full explanation. Among subjectively evaluated areas of health, impairment of mobility, as measured by the domains of mobility and activities of daily living scores on the PDQ 39, were least related to depression. More influential were the patients' impression of cognitive impairment and of stigmatisation. Even if a depressed patient views his quality of life worse than a non-depressed patient, the greater impairment of subjective cognitive and social functioning emphasizes the importance of these factors for the patients' well-being beyond the degree of physical impairment. 
Overall, in this study depression in PD was found to be the result of a multifactorial process, with objective impairment explaining only a comparatively small percentage of depression scores. Disability accounted for a greater proportion of the variability of depression scores, but the best predictor of depression was the patients' handicap in multiple areas, as measured by a health-related QoL instrument. Handicap alone predicted more than $50 \%$ of the variance of depression scores (see figure 5). A strong correlation of depression with subjectively evaluated handicap in the absence of correlation with measures of disease severity supports the notion that depression is at least partly a reaction to the impact of the disease on the individual's QoL. In contrast, Tandberg et al (Tandberg, 1997) concluded from their population-based study of depression in patients with PD that depression is a primary consequence of brain dysfunction. However, in their study, the only parameter assessing environmental factors was nursing home placement, and the patients' perceptions were not considered. I therefore propose that without disputing the known biochemical changes that may contribute, the patients' own personal circumstances and perception also greatly influence the occurence of depression in PD. The clinical differences between depressed and non-depressed patients (table 20) emphasise the complexity of this model, and suggest that subgroups of patients such as those with postural instability and cognitive decline may be more predisposed to developing depression. In conclusion, patients with cognitive impairment or postural instability, and those with recent deterioration, were more likely to be depressed than those without these disease factors. However, depression in patients with PD appears to be multifactorial, and strongly influenced by the individual patient's perception, rather than being an independent result of the disease process itself or a pure reflection of the patient's impairment. In practical terms, this underlines the importance of addressing the social and personal circumstances and the expectations and perceptions of a patient with PD who is depressed, and of treating his or her depression independently rather than restricting treatment to objective improvement of motor symptoms of PD. 


\subsection{The clinical subtypes of Parkinson's disease}

\subsubsection{Background}

For many years PD was considered a homogeneous disease characterized by levodopa responsive parkinsonism in the absence of additional neurological abnormalities and the finding of Lewy bodies at post mortem examination (Duvoisin, 1989). Its varying clinical expression has however recently led to the concept of subtypes of this disorder with the possibility of different pathogenic mechanisms (Jankovic, 1990; Duvoisin, 1989; Calne, 1989). In clinic-based samples of patients with PD differences have been found for instance between those with predominantly akinetic-rigid and tremor-dominant PD (Jankovic, 1990; Zetusky, 1985), patients with younger and older onset of disease (Kostic, 1991), those with tremor or bradykinesia at onset (Huber, 1988), between genders (Lyons, 1998), and those with and without distinct clinical features (Starkstein, 1989; Mayeux, 1992; Goetz, 1988). However, many of these variables may be related to each other, such as age at onset and age at examination, occurrence of depression and dementia, tremor at onset and tremordominant disease, or dementia and older age. In addition, patients assessed in these studies were derived from specialist clinics, or clinical trial populations (Jankovic, 1990), which may not be representative of the overall population of patients with PD. In order to overcome these limitations this population-based sample of patients with PD was analyzed according to the proposed clinical subtypes, and a subsequent cluster analysis with these variables performed to assess the existence of different subtypes in the overall population of patients with PD.

\subsubsection{Methods}

All patients diagnosed with possible or probable PD were included $(n=128)$. Depression was assessed with the Beck Depression Inventory (BDI; Beck, 1961) using a cut-off of 17 (18 or more indicating depression), and dementia with the mini mental state examination using a cut-off score of 24 (Folstein, 1975). The tremor-dominant subtype of PD was defined as patients with a ratio of tremor to bradykinesia score (rigidity and postural instability subscores from the UPDRS motor scale and history) $>0.5$, and the akinetic-rigid subtype as patients with a ratio of $<0.5$ (modified from 
Jankovic, 1990). In addition, those with age at onset before and after the mean age of onset (66 years), and those with current age younger or older than the mean current age (72 years) were compared. Rate of progression was calculated as disease severity divided by disease duration in years, and is henceforth referred to as rate of progression. Fast rate of progression was defined as a higher than the mean progression rate, and slow rate of progression as one lower than mean. Subjective response to levodopa was divided in poor or none $(<25 \%)$, moderate (26-50\%), good $(51-75 \%)$, and excellent (>75\%). Axial scores were derived from UPDRS items 27 to 30, tremor scores from items 21 to 22, and akinesia scores from item 19 and 23 to 26 .

The overall group of patients was divided according to the subtypes previously suggested, i.e. akinetic-rigid vs tremor-dominant $\mathrm{PD}$, slow vs fast progressors, according to symptom at onset, onset age, current age, those with and without dementia, depression, hallucinations or a family history of PD and between genders.

\subsubsection{Statistical analysis}

Due to the multitude of comparisons in this analysis only differences with $\mathrm{p}<0.005$ were accepted as statistically significant, but differences with $\mathrm{p}<0.05$ are given as marginally significant.In order to identify homogenous clusters in the overall sample of patients, an agglomerative hierarchical cluster analysis with squared euclidean distances using Ward's minimum variance was performed. The number of appropriate homogenous clusters was determined by the largest step in the squared Euclidean distance as a stopping rule. Non-hierarchical $k$-means cluster analysis was then performed to group patients according to their characteristics. Post hoc comparisons of

patients in the identified clusters were performed with the same statistical tests used for comparison of the proposed subtypes. All analyses were performed on SPSS software.

\subsubsection{Results}

Among the 128 patients $52.3 \%$ were male. The average age was 72 (SD 10.9), age of onset 66 (SD 14.3), and disease duration 6 (SD 4.6) years. 


\subsubsection{Young ( $<66$ years) vs old age at onset ( $>66$ years)}

Patients with onset of symptoms before age 66 were still significantly younger than those with onset after age 66, and but also had had symptoms for a longer time (table 23). Since disease severity was similar between the groups, rate of progression was marginally slower in patient with younger onset. Patients with younger onset also had marginally significantly higher rates of fluctuations and dyskinesias, lower rates of postural instability, and received higher doses of levodopa.

\subsubsection{Symptom at onset}

Onset with slowness or rigidity, as reported by the patients, was marginally significantly associated with a more frequent family history of parkinsonism, earlier onset of dyskinesias after symptom onset, higher disease severity with greater disability, more frequent difficulties with speech, freezing and falls (all $\mathrm{p}<0.05)$, but similar disease duration and age compared to onset with tremor. Dyskinesias, fluctuations and cognitive scores did not differ between the groups.

\subsubsection{Akinetic-rigid vs tremor-dominant $P D$}

Patients with the akinetic-rigid subtype of PD (73\%) had significantly greater disease severity and disability, as measured by the Hoehn and Yahr and the Schwab and England scale, than patients with tremor-dominant parkinsonism. However, they also had a slightly longer disease duration, indicating that patients with initially tremordominant PD later on developed more severe bradykinesia and axial features. Thus, the rate of progression in the overall sample was only marginally faster than in patients with akinetic-rigid subtype (table 24). As might be expected, tremor was the first symptom at onset in a slightly higher proportion of patients with tremor-dominant PD. Patients with the akinetic-rigid subtype also had marginally worse depression scores and quality of life scores, and higher rates of fluctuations, freezing and postural instability than those with tremor-dominant PD.

\subsubsection{Slow vs fast progression}

Patients with fast disease progression had greater disability and a marginally higher disease severity than those with slow progression despite a significantly shorter 
disease duration (table 25). They also had a significantly older age at onset, longer time from onset to initiation of levodopa treatment, used higher doses of levodopa, and tended to be older, to be demented and to have higher axial scores.

\subsubsection{Young (<72 years) vs older age ( $>72$ years)}

Patients with older current age also had older age at onset $(\mathrm{p}<0.0001)$, and had lower cognitive scores $(\mathrm{p}<0.0001)$. Older patients had a slightly shorter disease duration $(p<0.01)$ but also marginally higher disease severity $(p<0.05)$ and disability $(p<0.01)$. Thus, the rate of progression was marginally faster in older patients than in younger patients $(\mathrm{p}<0.05)$. On the other hand, time from symptom onset to first onset of dyskinesias was marginally longer in patients with older age $(p<0.05)$. Older patients also tended to have higher axial scores $(\mathrm{p}<0.01)$, reported falls marginally more often $(\mathrm{p}<0.05)$, received marginally lower doses of levodopa $(\mathrm{p}<0.05)$, and tended to be female $(\mathrm{p}<0.05)$.

\subsubsection{Gender differences}

Women were significantly older $(p<0.0001)$, tended to received lower doses of levodopa $(p<0.05)$ and tended to have worse quality of life $(p<0.05)$ than men. There were no other differences between the genders with regard to disease severity, disability, cognitive impairment or the occurrence of fluctuations or dyskinesias.

\subsubsection{Family history of parkinsonism}

Patients who had a first or second degree relative with parkinsonism (17\%) had significantly worse Hoehn and Yahr scores $(\mathrm{p}<0.005)$ than those without a family history of parkinsonism. However, as disease duration was also slightly longer in patients with a family history, a difference in disease progression failed to reach statistical significance. Patients with a family history of parkinsonism tended to have fluctuations and dyskinesias more often (both $\mathrm{p}<0.05$ ). Age at onset was not different between those with and without a family history of parkinsonism.

\subsubsection{Patient with and without dementia}


Patients with a mini mental score of 24 or less $(18 \%)$ had more severe disease and disability, had worse axial scores and poorer quality of life (all $\mathrm{p}<0.0001$ ). As disease duration did not differ significantly between demented and not demented patients, rate of progression was faster in patients with dementia $(p<0.0001)$. Patients with dementia also tended to be older, to have depression, postural instability, urinary incontinence or urgency more often than those without dementia, and to have lower tremor scores (all $\mathrm{p}<0.05$ ).

\subsubsection{Patients with and without depression}

Patients with BDI depression scores of more than 17 had more severe Schwab and England disability scores and worse quality of life scores (both $\mathrm{p}<0.0001$ ) than patients without depression. Depressed patients also more often tended to have falls $(p<0.01)$, greater postural instability, the akinetic-rigid subtype of $P D$, hallucinations and dementia, and take higher doses of levodopa (all $\mathrm{p}<0.05$ ) than not depressed patients. They did not differ with respect to age, age at onset or rate of progression.

\subsubsection{Patients with and without a history of hallucinations}

There were no significant differences between patients with $(19.5 \%)$ and without hallucinations. However, patients with hallucinations tended to have more severe disease, to have longer disease duration, be depressed and have worse quality of life than those without hallucinations (all $\mathrm{p}<0.05$ ).

\subsubsection{Cluster analysis}

In order to identify subgroups of patients with PD who share similar characteristics within the overall sample, a cluster analysis entering all variables that had been shown to be significantly different between groups was performed. Hierarchical cluster analysis indicated that a 3-cluster solution provided the most appropriate grouping of patients. After 3 iterations non-hierarchical k-means cluster analysis arrived at 3 clusters whose characteristics are shown in table 26.75 patients (58.6\%) formed cluster one, 37 patients $(28.9 \%)$ belonged to cluster 2 and 16 patients $(12.5 \%)$ to cluster 3 . The clusters differed significantly in all variables except axial scores and time from onset to initiation of treatment with levodopa. 
Mean disease and treatment duration was significantly longer in group 3 than in group 2 (both $\mathrm{p}<0.001$ ), and marginally longer than in group 1 (both $\mathrm{p}<0.05$ ). Patients in group 3 also had more severe disability compared to group $1(\mathrm{p}<0.001)$ and group 2 $(p<0.01)$, and took higher doses of levodopa than those in the other groups (both $\mathrm{p}<0.0001)$. However, disease severity was only marginally greater than in group 1 $(\mathrm{p}<0.05)$ and similar to group 2 . Thus, rate of progression was significantly slower than in group 2, and tended to be slower than in group 1 ( $\mathrm{p}<0.005$ and $\mathrm{p}<0.05$, respectively). The patients in group 3 also were significantly younger at onset (both $p<0.0001$ ), and were still younger than those in the other groups (both $\mathrm{p}<0.0001$ ). Despite the longer disease duration, they did not differ from the other groups with respect to mean depression or quality of life, and had better cognitive scores $(p<0.001)$. On the other hand, they had fluctuations more often than patients in group $2(p<0.001)$ and they tended to have more fluctuations than those in group 1.

Patients in group 2 had the oldest age at onset $(\mathrm{p}<0.005$ and $\mathrm{p}<0.0001$ compared to group 1 and 3, respectively) and were still older than those in both other groups $(p<0.005$ and 0.0001$)$. Although mean disease and treatment duration was shorter (both $\mathrm{p}<0.005$ compared to group 1 and $\mathrm{p}<0.001$ compared to group 3), mean disease severity was more severe than in group $1(\mathrm{p}<0.01)$, and similar to group 3 . Thus, rate of progression was faster than in both other groups (both $\mathrm{p}<0.005$ ). Patients in group 2 were taking lower doses of levodopa (both $\mathrm{p}<0.0001$ ), had worse cognitive scores than those in both other groups $(\mathrm{p}<0.001)$, worse quality of life than those in group 1 $(p<0.01)$, and tended to have worse disability than in group $1(p<0.05)$, but had fluctuations less frequently than those in both other groups (both $\mathrm{p}<0.001$ ).

Patients in group 1 were in between groups 2 and 3 with respect to most variables. Compared to those in group 3, patients in group 1 had older age at onset and age (both $\mathrm{p}<0.0001)$. They had slightly shorter disease duration $(\mathrm{p}<0.05)$, took lower doses of levodopa $(p<0.0001)$, had less disability $(p<0.001)$, and tended to have less severe disease $(\mathrm{p}<0.05)$. They had, however, slightly faster disease progression $(p<0.05)$. Compared to group 2, they were younger and had younger age at onset (both $\mathrm{p}<0.005)$, took higher doses of levodopa $(\mathrm{p}<0.0001)$, had better mini mental scores $(p<0.001)$ and had slower disease progression $(p<0.005)$. The groups did not differ with 
respect to percentage of the akinetic-rigid or tremor-dominant subtype, depression scores, to symptom at onset, hallucinations, family history of parkinsonism or gender.

\subsubsection{Discussion}

The results of this analysis of a population-based sample of patients with PD support the hypothesis of different subtypes of PD. In the overall sample three subgroups of patients were identified: 1) Patients with a young onset age in whom disease progression was slower than in the other groups, and who were also younger at the time of examination. Due to longer disease duration they were more severely disabled than older patients, used high doses of levodopa and had more frequently fluctuations. However they also had better cognitive scores than older patients, and rate of progression was slower. 2) Patients with an older age at onset in whom disease progression to more advanced stages was fast and who had low cognitive scores, but who had developed motor fluctuations more rarely than the younger patients. 3) An intermediate group with age at onset, current age, levodopa dose, and rate of disease progression in between group 1 and 2 . Thus the main factor discriminating between patients with different characteristics was age at onset.

This differentiation predominantly by age at onset was supported by the separate comparison of patients with younger vs older age at onset, which showed significant differences in disease progression. Patients with a young onset age also tended to develop motor complications more frequently than those with older onset age, whereas those with older age at onset tended to have developed postural instability more frequently. Patients with older current age (which correlated with age at onset) were additionally more likely to have dementia, tended to have falls, and progress more rapidly, but had developed motor fluctuations later. These peculiarities of PD in older patients may be explained by additional brain damage in older patients, which renders them more prone to falls, cognitive impairment and faster progression, but also prevents dyskinesias and fluctuations. The faster progression may in addition also be partly due to the scaling of the Hoehn and Yahr scale, in which postural instability, a common finding in elderly people (Waite, 1996), is an important factor. Most other studies similarly found clinical differences between patients with young vs old onset (Kostic, 1991; Zetusky, 1985), whereas pathological studies did not find a correlate 
explaining these differences, apart from greater extent of depletion of dopaminergic neurons (by $24 \%$ ) but no differences in the basic Lewy body pathology in patients older age, but longer disease duration (Gibb, 1989b).

Demented patients not only had a faster rate of progression, but also tended to have depression, postural instability, urinary incontinence and to have lower tremor scores than those without dementia. Their disability was greater as well their quality of life poorer compared to those without dementia. This confirms results from other studies that found that dementia in PD is associated with increasing disease severity, age and depression (Mayeux, 1981; Ebmeier, 1991; Huber, 1988; Zetusky, 1985). Jankovic et al (Jankovic, 1990) have suggested that the tendency of demented patients to have greater postural instability and lower tremor scores may be explained by a relatively greater degree of cholinergic deficit causing dementia in PD as well as postural instability, thus balancing out the cholinergic preponderance typically expressed as parkinsonian tremor.

The identified subgroups were however not distinguished by symptom at onset, gender, history of hallucinations, family history of parkinsonism, depression and predominance type. Nevertheless, those with the akinetic-rigid subtype were more severely diseased, with more severe features of advanced disease, although this may in part have been a reflection of the increasing akinesia and axial involvement in advancing disease. However, they tended to have progressed more rapidly than those with tremor-dominant PD, confirming previous reports (Jankovic, 1990; Zetusky, 1985). The results of some (Hershey, 1991), but not other studies suggested that tremor at onset is a prognostically positive factor (Huber, 1988; Röhl, 1994). In this study, patients with bradykinesia or rigidity at onset tended to be more severely diseased after a similar disease duration, but this difference in rate of progression did not reach statistical significance. Patients with a family history of parkinsonism tended to have motor complications more often than those without, and a difference towards faster disease progression just failed to reach significance. Although these findings hint at a genetically determined different type of parkinsonism, these differences were not significant. Hallucinations were not associated with a specific subtype of PD, but tended to occur in advanced disease and were associated with depression and poorer quality of life. Depression, on the other hand, was not strongly associated with disease 
severity, but depressed patients were more disabled and had worse quality of life than those without depression. They also tended to have postural instability and falls, dementia and hallucinations more often than those without depression. This is in accordance with previous studies that have suggested that depression is not directly related to increasing motor disability (Starkstein, 1989; Santamaria, 1986). Unlike in other studies (Santamaria, 1986), however, there was no evidence to suggest a separate subtype of patients with depression and PD within the overall group of patient with PD.

The existence of different subtypes of PD has previously been examined by comparison of different groups of patients (Huber, 1988; Hershey, 1991; Zetusky, 1985; Kostic, 1991; Mayeux, 1981). However, only few studies evaluated the existence of different subtypes by comparing patients with or without a variety of different distinctive characteristic in the same group of patients. Jankovic et al (Jankovic, 1990) used the opportunity of a very large database of patients with PD in the DATATOP study to explore the possibility of different subgroups in this population. They found evidence that patients with young onset functioned cognitively better than those with older onset and had slower disease progression, that patients with rapid progression not only were older at onset, but more often had bradykinesia, postural instability and gait difficulties at onset than those with slow progression, and that patients with akineticrigid $\mathrm{PD}$ had greater subjective intellectual, motor and occupational impairment than those in the tremor-dominant subtype after a similar disease duration. These findings are similar to those in this study, despite the fact that the patients were selected from clinic-based samples for eligibility in a treatment trial and had similar disease severity at inclusion in the study. Fast progression in that study, however, was not related to cognitive decline, indicating that motor and cognitive decline are not necessarily associated with each other. However, as the authors point out, DATATOP excluded patients with actual cognitive impairment, and these results may thus not be generalisable to the overall population of patients with PD, including those with actual dementia. In this study, demented patients had progressed more rapidly than not demented patients, although there was only a marginally significant difference between the proportion of demented patients between fast and slow progressors. This finding indicates that development of dementia is an important factor for faster motor progression of disease, confirming results by Mayeux (1981), Huber (1988), and 
Ebmeier (1991). The relation of several variables in that and this study suggests that several clusters of patients exist within the overall population, which could be confirmed using cluster analysis. A similar approach to classify patients within the overall group of patient with PD has been used by Graham et al (Graham, 1999). Using non-hierarchical cluster analysis with an arbitrarily chosen number of 5 clusters, these authors found that a clinic-based sample of patients with PD fell into 3 groups with short and 2 with long disease duration, which differed in motor and cognitive scores. One group with short disease duration also had older age at onset and more severe impairment of motor and cognitive scores. The authors concluded that there are three subtypes of PD, predominantly determined by cognitive impairment: Those with mean motor impairment only, those with motor and cognitive impairment, and those with an older age at onset and rapid progression of motor and cognitive performance. In agreement with this patients in the cluster with the oldest age at onset had the most rapid disease progression and tended to have worse cognitive scores. However, in this study cognitive state was not the determining variable differentiating the subgroups. The most likely explanation for this difference is that in this study only a screening tool for dementia (the mini mental state examination) was used to diagnose clinically significant dementia, whereas the previous study performed cluster analysis predominantly based on a variety of cognitive scores. It was thus weighted towards cognitive scores, whereas in this study clinically significant cognitive decline was only one of a variety of demographic and clinical variables. In addition, only variables that had been shown to differ between postulated subgroups of PD were entered in the cluster analysis to minimize chance findings. Furthermore, prior to k-mean cluster analysis hierarchical cluster analysis was performed to determine the number of naturally occurring subgroups within the sample and avoid the necessity of arbitrarily choosing the number of clusters.

Due to the inherent limitations of a cross-sectional population-based sample, this study has some limitations. Clinical diagnosis was not confirmed with postmortem examination and, thus, the inclusion of patients with atypical forms of parkinsonism cannot be excluded with certainty. However, the main emphasis of the overall study was the differentiation of different types of parkinsonism, and I therefore believe that 
the rate of diagnostic accuracy was comparatively high. In future studies an important aspect would be to correlate pathological differences with the clinical subtypes in order to explain the differences found between the subtypes. Patients with mild disease, who have not yet come to medical attention, may also have been underascertained, and patients with dementia, on the other hand, may have been more likely to have declined participation, and were excluded if dementia was present before onset of parkinsonism. However, I endeavoured to obtain as complete patient ascertainment as possible, and the participation rate in this study was high (84\%). Therefore, it is likely that the patients in this sample are more representative of the overall population of patients with PD than previous studies undertaken in selected, clinic-based samples.

I conclude that different subtypes of PD exist. The most important factor for subclassification of patients with PD is age at onset, but other influential factors differentiating patients within this population were presence of dementia, akinetic-rigid or tremor predominance, current age and rate of progression, which were often associated with age at onset. Symptom at onset, and occurrence of hallucinations, gender, family history or presence of depression, on the other hand, appear to be relatively independent factors, which are not associated with a specific subtype of disease. 


\subsection{Young Onset Parkinson's Disease - Clinical features, natural history and mortality}

\subsubsection{Background}

PD starting before the age of 40 years is rare. However, if the disease starts at an early age, as discussed above, it not only has a longer duration, but also appears to have a number of characteristics which differentiate it from the overall sample of patients with PD. In order to study the clinical characteristics and prognosis of PD starting at a young age, a large group of patients with such early onset of PD was therefore investigated in a separate group from the population based sample of patients with PD.

Patients who develop parkinsonian symptoms or signs between 21 and 40 years have arbitrarily been called 'young onset Parkinson's disease' (YOPD), and those in whom they start before age 21 'juvenile parkinsonism' (JP; Quinn, 1987). Juvenile parkinsonism probably represents a mixture of different pathologies (Horowitz, 1975; Horowitz, 1917; Van Bogaert, 1930; Davison, 1947; Yokochi, 1993; Gibb, 1988; Mizutima, 1991; Takahashi, 1994; Yoshimura, 1988). In contrast, YOPD is thought to usually represent the lower end of the age spectrum of Lewy body PD since most pathological studies have revealed typical Lewy body pathology (Quinn, 1987; Gibb, 1988; Yokochi, 1993; Mizutima, 1991). However, a number of studies have reported differences between YOPD and older onset PD in terms of clinical features, progression of disease, latency to time of first appearance of levodopa-related motor complications, and prognosis (Giovannini, 1991; Gibb, 1988; Kostic, 1991), giving rise to the assumption that there may be two different subtypes of $\mathrm{PD}$, one a predominant motor disorder with an earlier age of onset, and one with greater mental deterioration and a later age of onset (Lieberman, 1979). Due to the rarity of the disorder, most studies have reported only small numbers of patients with YOPD from whom it is difficult to draw firm conclusions.

Additionally, no prospective studies have been performed to assess the prognosis of early onset PD.

The clinical data and mortality of a large group of patients with apparent idiopathic PD starting before the age of 40 , and of a subgroup of 60 prospectively studied patients who were reported 10 years ago (Quinn, 1987) are reported here. Due to the rarity of this subgroup, this group was investigated independent of the population-based sample of patients with $\mathrm{PD}$.

\subsubsection{Patients and methods}

All patients who developed apparent idiopathic parkinsonism before age 40 were identified among all patients with parkinsonism seen by Professor Niall Quinn 
(NQ) and the late Professor David Marsden (CDM) during the last 17 years. For inclusion in the study at least two of three cardinal features of parkinsonism (tremor, rigidity and bradykinesia) had to be present. Patients were excluded if they had a history of encephalitis, repeated head trauma or treatment with neuroleptics, or presented additional neurological features incompatible with idiopathic PD such as pyramidal signs, supranuclear gaze palsy or cerebellar ataxia. One patient who was demented at presentation was excluded. Wilson's disease was excluded in all cases. A subgroup of 60 patients, first reported 10 years ago (Quinn, 1987), were analysed separately. These patients had either died or had been seen during the previous 5 years. For the separate prospective analysis of this subgroup, the above inclusion and exclusion criteria were met at the time of the first report, but subsequently two of these patients had their diagnosis revised and were excluded from the overall analysis.

Approval for the study was obtained from the Joint Medical Ethics Committee of the National Hospital for Neurology and Neurosurgery and the Institute of Neurology. The case notes of all patients were examined and information on symptoms, signs and age at onset of parkinsonism, a history of family members with parkinsonism, time that 1-dopa was started and response to treatment, occurrence of, and latency to, motor complications of treatment, and features of disease progression were recorded. Most patients continued to be followed by NQ or CDM at the National Hospital for Neurology and Neurosurgery. All living patients were contacted and asked about parkinsonism in first or second degree family members, current symptoms, history of psychiatric side effects of medication, falls, degree of disability, first occurrence of dyskinesias and motor fluctuations, and asked to self-report the Schwab and England disability scale which was read to them. Their spouses acted as additional informants. If they agreed to participate, a cognitive test validated for telephone administration (Brandt, 1988) was administered by telephone. This cognitive status questionnaire has a maximum score of 41 , and a cut-off score for clinically significant cognitive impairment (henceforth considered as dementia) of 30 or less. Vital status of those who could not be contacted was ascertained from the National Health Service (NHS) Central Register, which keeps records of all individuals in the UK registered with a health authority. Cause of death was ascertained from death certificates and classified according to the World Health Organisation (WHO) rules, and care was taken to follow rule 3. For example, if bronchopneumonia was given as the sole cause of death in part I and PD was mentioned in part II, PD was coded as cause of death. Statistical analysis of categorical data was performed using Chi-Square and Fisher's exact test. T-test for independent samples was used for comparison of interval data between groups. Correlation of interval data was performed with Pearson 
correlation, and discriminant analysis was used for classification of dementia. The relative mortality experience of this cohort was compared to that from the general population. This was done by using indirect standardisation to calculate an expected number of deaths. In this way it was possible to calculate a standardised mortality ratio (SMR) comparing observed to expected deaths (Clayton, 1993). Because there have been marked secular changes in mortality, it was essential that standardisation used the appropriate age, sex, and cohort specific mortality rates. Therefore, the person-years of follow-up for each observation were partitioned into 10-year age bands and 5-year time periods from 1941 to 1996 . In this way, the routine mortality data could be used to calculate rates for the same groups for all of England and Wales. 95\% confidence intervals (CIs) were calculated using the Poisson distribution. Significance testing for the clinical sub-groups was carried out using the maximum likelihood estimate of the rate ratio. All these survival analyses were carried out using STATA software (Clayton, 1993).

\subsubsection{Results}

Ten patients developed their first symptoms of parkinsonism before the age of 21 (JP) and 139 between the age of 21 and 39 (YOPD, figure 6). In the subgroup of 60 patients who were reported previously 4 patients had JP and 56 YOPD. The characteristics of all patients are shown in table 27. The JP patients will be described case by case, while those with YOPD will be considered as a group.

\subsubsection{JP group:}

Recent follow-up status was obtained in all patients. Three patients had died and 7 patients were still alive. Current age or age at death in this group ranged from 10 to 62 years. There was a male predominance of $4: 1$. Early development and birth history was normal in all patients. First symptoms appeared at the age of 5 to 19 years with dystonia in $6(60 \%)$, tremor in $5(50 \%)$, and an akinetic-rigid syndrome in $2(20 \%)$ patients. $50 \%$ of patients had at least one first degree relative with parkinsonism, in all of whom the disease had started before the age of 40 . All but one patient who received l-dopa had an excellent initial motor response, but this rapidly declined in one of them. Dyskinesias and motor fluctuations developed in all patients who received l-dopa, after a median of 6 months treatment.

Case 1 (sporadic) was a 28 year old patient who had presented with dystonia at the age of 7 years and had initially been diagnosed as dopa-responsive dystonia. However, after one year of 1-dopa treatment he developed wearing-off and mild dyskinesias and his ${ }^{18}$ F-Dopa PET scan revealed severely reduced uptake in caudate and putamen bilaterally. He nevertheless continues to be dramatically improved by 1-dopa and leads an active life. Case 2 (sporadic), who had first 
developed a tremor at the age of 13 , also had a peripheral and autonomic neuropathy. Chronic treatment with 1-dopa was not tolerated because of postural hypotension and painful dystonic spasms. A PET scan revealed markedly decreased striatal ${ }^{18}$ F-Dopa uptake. Genetic testing was positive for a mutation in the Parkin gene. Case 3 (sporadic) suffered from severe concomitant depression, and case 4 developed dementia at age 33. His father had died at the age of 48 after nine years of parkinsonism. Genetic testing for the Parkin gene was negative. Case 5 was a 32 year old Madeiran patient with parkinsonism since the age of 17 . He has a sister who also has juvenile parkinsonism. They were the product of a consanguineous marriage, and their father was also said to have a tremor. Motor fluctuations and involuntary movements developed early and were later severe enough to warrant pallidotomy. There was no dementia, but he suffered psychotic side effects with dopaminergic medication. Among other investigations, genetic analysis for the SCA 3 mutation was negative, but he tested positive for the Parkin gene. Case 6 (sporadic) was a 42 year old patient, who had had parkinsonism since the age of 18 . Her response to 1-dopa was excellent, but she developed severe motor fluctuations and dyskinesias within the first two years. She had no cognitive decline, had never experienced confusion or hallucinations and had only mild impairment of balance. Case 7, who reported a paternal uncle suffering from parkinsonism, and several of his siblings to have parkinsonism or a tremor, had predominantly dystonic peakdose dyskinesias. He was in the low normal range of intelligence (verbal and performance IQ 81), but there was no cognitive decline. However, he has experienced two episodes of manic psychosis on dopaminergic medication. Genetic testing for the Parkin gene was positive. Case 8 (sporadic), the youngest patient in this series, had developed gait problems at the age of 5. L-dopa improved his akinetic-rigid syndrome dramatically but he experienced wearing off fluctuations immediately and dyskinesias within nine months. He also suffered painful dystonic spasms lasting up to 5 hours. All investigations, including copper studies, a CT scan, bone marrow, liver and rectal biopsies, and plasma and urinary amino acids were negative except for bilateral non-specific slow wave activity in his EEG with multifocal spike-wave activity. He died at the age of 10 years. Case 9 noted the first symptoms of parkinsonism at the age of 17 . He had two sisters and one brother who had also developed parkinsonism in their twenties or earlier. He died of a bronchial carcinoma at the age of 62 years after 45 years of disease. Case 10 was a patient who had an akinetic-rigid syndrome when he was first seen at age 35 and had first developed 'vibrations' at the age of 17 years. Five out of 8 of his siblings, including one sister, were similarly affected by history. He responded dramatically to 1-dopa but developed marked choreiform involuntary movements in response to treatment. He continued to work as a lecturer until the age of 61 and died at age 72 . Post- 
mortem examination of the brain was not performed in any of these three patients who had died. The standardised mortality ratio (SMR) in this group was 3.1 (95\% CIs 0.64-9.1) compared to the normal population.

\subsubsection{YOPD group:}

109 patients were still alive, 23 had died and 7 patients were lost to follow-up. I was able to contact and administer the short cognitive test to 98 patients $(90 \%$ of those alive). The remaining 11 living patients did not reply or declined to participate.

7.6.3.2.1. Onset: Age and clinical features at onset are given in table 27. $16 \%$ of patients had at least one first (12 patients, $8 \%$ ) or second (10 patients, $7 \%$ ) degree relative with parkinsonism. Two patients had two affected first or second degree relatives with parkinsonism.

\subsection{Complications of l-dopa treatment. Over $25 \%$ of patients developed} complications within one week of commencing treatment and over $40 \%$ within 6 months. After 5 years of treatment with 1-dopa $91 \%$ of 99 patients had developed dyskinesias and $92 \%$ motor fluctuations, and after 10 and 12 years, respectively, all patients were suffering these side effects (figure 7). A large number of patients who could be contacted (36 out of 102, 35\%) had undergone stereotactic surgery for relief of these complications (4 fetal transplantations, 3 pallidal stimulations, 23 pallidotomies, 5 thalamotomies, and one patient who had undergone thalamotomy and pallidotomy). However, many of these patients came under our care specifically because they had been referred for these procedures. Psychiatric side effects (psychosis, confusion or hallucinations) had occurred in $49 \%$ of patients at some point in their disease. These patients were significantly older at time of contact (median age 53 vs 47 years, $\mathrm{p}<0.001$ ) and had a longer disease duration (20 vs 14 years, $\mathrm{p}<0.001)$. They also had a significantly lower telephone mini mental score (median 31, SD 6.0) compared to the remainder (median 35, SD 3.9, p<0.001). There was no significant difference in history of psychiatric side effects between patients below and above the age of 60 across the sample as a whole $(50 \%$ and $68 \%$, respectively). However, only $10 \%$ of those who had had the disease for less than 10 years had experienced psychotic side effects.

7.6.3.2.3. Current state: Median age at the time of investigation or age at death was 51 (33 - 78) years and median disease duration was $18(2-43)$ years. All patients had akinesia and rigidity, 68 (78\%) tremor and 60 (67\%) dystonia (usually treatment-related). Hoehn \& Yahr stage was 4 or 5 in $57 \%$ of patients in the off state, but in only $4 \%$ of patients in the on state. Similarly, the Schwab and England score was $50 \%$ or lower in $13 \%$ of patients in the off state, but in only $5 \%$ of patients in the on state. Falls had occurred in $50 \%$ of patients, but in only $5 \%$ of 
patients with a disease duration of less than 10 years. Fallers were significantly older (median age 54 vs 47 years, $\mathrm{p}<0.001$ ) and had had PD for longer (median 21 vs 11 years, $\mathrm{p}<0.001$ ). As far as it was possible to ascertain, in $81 \%$ falls were related to impairment of balance, in $75 \%$ to freezing episodes, and in $29 \%$ to dyskinesias. Many patients experienced falls related to two or more of these features.

7.6.3.2.4. Dementia: Cognitive functions, as assessed by the brief telephone cognitive test (with a maximum score of 41 ), was determined in 98 patients $(90 \%$ of those alive). 19 patients (19\%) were below the cut-off score for dementia of 31/41. Characteristics of patients with and without dementia are shown in tables 28 and 29. Demented patients were significantly older at the time of assessment with a longer disease duration. Dementia was uncommon in patients below age $60(13 \%)$, but was found in $43 \%$ of those aged over 60 . Likewise, dementia was rare in patients who had PD for less than 10 years, but more frequent in those with a longer disease duration.

In an attempt to understand the relationship between dementia, current age and disease duration a series of partial correlations were calculated. When adjusted for current age using Pearson's partial correlation, disease duration no longer correlated with the mental status score $(r=0.46, p=0.27)$. However, when the opposite correlation was carried out, age still significantly correlated with the mental status score $(r=0.24, \mathrm{p}<0.05)$. Of those with PD for 20 to 43 years, $66 \%$ ( 25 patients) had no evidence of cognitive decline. While the strongest correlation was between current age and cognitive status, demented patients were also significantly more likely to be female $(\mathrm{p}<0.01)$, to have experienced psychiatric side effects during the course of treatment $(\mathrm{p}<0.001)$ or falls $(\mathrm{p}<0.05)$ and to have lower 'on' $(p<0.01)$ and 'off' $(p<0.01)$ Schwab and England scores. They also tended more often to have a positive family history of parkinsonism, although this effect was not significant. Men and women did not differ in age, disease duration, onset age, and all other investigated parameters, but psychiatric side effects of medication, falls, and disability scores all correlated with age and disease duration.

Using discriminant analysis, a number of variables that could be derived from information at onset of parkinsonism were tested for their predictive value for the subsequent development of dementia. Among the factors known at onset, female sex and positive family history of parkinsonism were useful clinical predictors of dementia (correct classification in $72 \%$, sensitivity $67 \%$, specificity $73 \%$, positive predictive value $36 \%$, negative predictive value $90 \%$ ). Patients with a relatively older age at onset within the YOPD group were also more likely to develop dementia but the difference was small ( 36 vs. 33 years) and not clinically useful. Initial response to l-dopa and symptom at onset were not useful predictors. 
The accuracy of prediction of dementia was further increased by considering the occurrence of psychiatric side effects on 1-dopa during the disease course. Together these variables correctly classified $82 \%$ of patients as demented or non-demented (sensitivity $71 \%$, specificity $84 \%$, positive predictive value $50 \%$, negative predictive value $93 \%$, tables $30 \mathrm{a}, 30 \mathrm{~b}, 31$ ).

7.6.3.2.5. Mortality: Vital status was known in 132 YOPD patients (95\%). 23 patients had died (median age at death 57.7 years and disease duration 22.5 years) and 109 patients were alive (median age 50.1 years, disease duration 16.9 years). The SMR was 2.1 (95\% CIs 1.4-3.2) compared to the normal population. An increased risk of death was found in four patients with a poor initial response to 1dopa $(p<0.001)$. There was also a tendency for patients with tremor at disease onset to die earlier $(\mathrm{p}=0.11)$. The factors sex and family history of parkinsonism were not predictive of mortality.

Median survival was 27 years for patients with disease onset of 36 to 39 years and 35 years for patients with disease onset from 22 to 35 years.

Cause of death: Death was considered directly related to parkinsonism in 15 patients $(65 \%)$. One patient with a disease duration of 15 years died after an episode of severe diphasic dyskinesias at the age of 51 . Two patients $(9 \%)$ died of neoplasms, four of ischaemic heart disease $(17 \%)$, one of gastric ulceration $(4 \%)$, and one of bronchopneumonia (4\%). A post-mortem examination of the brain had been performed in five patients and revealed brain stem Lewy body disease in all of them. In the father of one further patient who also had YOPD, Lewy body PD was also confirmed pathologically.

\subsubsection{Prospective subgroup of 60 patients}

7.6.3.3.1. Juvenile parkinsonism._In the four patients with $\underline{\mathrm{JP}}$, current age or age at death was 43.5 (35-62) years and disease duration 25.5 (16-45) years. All patients had at least one first degree relative with parkinsonism. No alternative diagnosis had been made in any of these patients, although one of them later developed dementia at the age of 33. Only one patient had died, at the age of 62. The SMR was 3.2 (95\% CIs 0.08-18).

7.6.3.3.2. Young Onset Parkinson's disease. In the YOPD group, 30 patients were still alive, 22 had died, and 4 patients were lost to follow-up. Current age or age at death in this group was 57 (35-78) years and median disease duration 21.5 (5-41) years. The diagnosis was changed in two YOPD patients. One patient, despite an excellent initial response and good ( $>50 \%$ improvement) persisting response to levodopa therapy, had developed early postural instability with falls, postural hypotension, respiratory stridor and urinary retention and incontinence with a highly abnormal sphincter EMG and died 5 years after onset of symptoms at the age 
of 39. A clinical diagnosis of MSA was made in life and confirmed pathologically after death (Wenning, 1994). Another patient who had had a partially 1-dopa responsive parkinsonism-dystonia syndrome since the age of 36 subsequently developed insulin-dependent diabetes, a peripheral neuropathy, and abnormal eye movements. Genetic analysis revealed that he had Machado-Joseph disease. The family history was initially thought to be negative, but it became eventually known that one sister had developed an ataxic syndrome with muscular atrophy, ophthalmoplegia and areflexia without parkinsonism from the age of 49 , and one brother and the father were also said to be affected. Of the remaining 54 YOPD patients, 21 had died after a median disease duration of 25 (11-38) years at an average age of 60 (48-71) years, whereas those still alive had had parkinsonism for 22 (14-41) years at a median age of $57(41-78)$ years. The mortality ratio of the YOPD group was 2.9 (95\% CIs 1.78-4.40) compared to the normal population.

\subsubsection{Discussion}

Most patients (73\%) in this study developed parkinsonian features between the age of 30 and 40 years, whereas JP was very rare (7\% of the whole group). This distribution is similar to that in other studies in Western populations but differs from the pattern in Japan where onset before the age of 21 is much more common (Yokochi, 1984). In Japanese studies familial parkinsonism was also reported to be present in a high percentage (up to $42.5 \%$ ) of patients. This may be partly related to the high levels of parental consanguinity in these series, since recessive early (usually juvenile) onset parkinsonism is particularly common in the Japanese families (Mizutima, 1991; Ishikawa, 1996). Studies in Western populations have reported familial occurrence of young onset parkinsonism in a rather lower percentage of 9.5 to $37.5 \%$ (Quinn, 1987; Ludin, 1989; Barbeau, 1982). The familial occurrence of PD in the YOPD group in this report (16\%) did not exceed that reported in the general PD population (Payami, 1994; Bonifati, 1995), indicating no greater genetic component in YOPD than in older onset PD (OOPD). However, the recent finding of a higher concordance of PD in monozygotic than in dizygotic twins in patients with young onset, which was not found in patients with older onset, suggests a greater genetic determination in YOPD (Tanner, 1997). Fourteen percent of YOPD patients had focal dystonia at onset of symptoms, a higher percentage than reported in OOPD patients, an observation which had previously been made in YOPD (Quinn, 1987; Gibb, 1988; Gershanik, 1986). There was a male predominance of 1.7:1 in the YOPD group, and of 4:1 in the JP group. Other groups have reported similar findings (Zetusky, 1985; Friedman, 1994; Pantelatos, 1993). In this young patient group this is unlikely to reflect referral patterns or access to health care facilities, and may therefore be a true finding. 
When patients are diagnosed with $\mathrm{PD}$ at a relatively young age the question of prognosis in terms of progression of motor symptoms, disability, preservation of mental function, and mortality is of particular interest.

Few studies have investigated mortality in YOPD. In a retrospective study of pathologically proven cases of 12 patients with YOPD (onset $<40$ years) and 22 cases with OOPD (onset $>70$ years) Gibb and Lees (Gibb, 1988) found that, although mean disease duration to death was 12 years longer in the YOPD group than in the OOPD group, difference in mean age at death was 30 years in favour of OOPD cases. Therefore, patients who develop PD at a young age will live in absolute terms for a longer period of time than older cases. However their life expectancy is reduced to a greater degree than older cases. Diamond et al. (Diamond, 1989) compared mortality in young and old onset PD patients to mortality in the general population and found that patients with onset before the age of 50 had a lower observed to expected mortality ratio than those who had developed the disease above the age of 59, supporting the notion that early onset carries a more benign prognosis. However, this difference was not significant. In contrast, mortality in YOPD was increased twofold compared to the normal population, which is similar to the expected to observed mortality ratio in the general PD population (Martilla, 1976; Bennett, 1996; Ben-Shlomo, 1995; Ransmayer, 1986). However, the number of patients who were referred specifically for consideration of stereotactic surgery, together with the possibility that other patients with a worse prognosis might have died before coming to our attention, might introduce a bias to this sample. This potential bias is avoided in the analysis of the smaller prospective subgroup of 54 YOPD patients, in whom mortality was threefold compared to the normal population. However, because of the wide confidence intervals, this result is still consistent with the mortality risk found in the general PD population. Four patients with a poor initial response to 1-dopa had a higher risk of early death, possibly suggesting a more aggressive, and perhaps different, disease in these patients. However, this number is too small to draw any useful conclusions. Patients with tremor at onset did not have a better prognosis than those with akinetic-rigid onset, which is in contrast to the finding of others (Hoehn and Yahr, 1967; Bennett, 1996; Ransmayer, 1986) who found a lower mortality in PD patients with tremor onset compared to those with akinetic onset.

Postural reflexes were preserved in almost all of the patients in this study for the first 10 years and even in the majority of patients who had had PD for 20 years. Similarly, disability as assessed by Schwab and England and Hoehn and Yahr scores was relatively low in both 'on' and 'off' states after a disease duration of 10 years, supporting the notion of slow disease progression in YOPD. Diamond et al (Diamond, 1989) similarly found that after 4 years of disease patients in the 
youngest of their three groups (age of onset less than 50 years) had significantly lower disability scores than in the oldest group (age of onset more than 59 years). This was mainly due to a difference in axial symptoms such as posture, gait, and postural reflexes. Using a different approach, Goetz et al (Goetz, 1988) found that the best factor to distinguish rapid from slow progressors was older age at onset. The studies by Ludin et al (Ludin, 1989) and Jankovic et al (Jankovic, 1990) both found that disease duration was longer in patients with earlier age of onset than in patients with late age of onset at the same level of disability, suggesting that disease progression was slower in earlier onset $\mathrm{PD}$. Only a minority of studies have found a more rapid progression in early onset patients than in old onset patients (Giovannini, 1991; Caraceni, 1985).

In contrast, motor fluctuations of response to 1-dopa and dyskinesias have consistently been found to occur more commonly, earlier, and to a more severe degree, in patients with a relatively young age at onset (Giovannini, 1991; Quinn, 1987; Gibb, 1988; Yoshimura, 1988; Gershanik, 1986; Friedman, 1994; Pantelatos, 1993). These earlier findings were confirmed in this study. After 10 years of disease duration almost all patients were experiencing motor fluctuations and dyskinesias, necessitating stereotactic surgery in many. More patients might have had stereotactic surgery had they lived to the renaissance of pallidotomy for treatment of 1-dopa induced dyskinesias.

A relatively high number of patients had experienced psychiatric side effects of treatment, even among those below the age of 60 years $(50 \%)$. Other authors (Giovannini, 1991; Friedman, 1994) found a much lower occurrence (5\%) of this complication of therapy, significantly less frequently than in their older onset groups (15\% and $43 \%)$. The difference between those findings and those of this study may partly be due to the fact that the history of these side effects was recorded after a much longer duration of disease (18 vs. 10.4 and 12.7 years). Thus, after a disease duration of up to 10 years only $10 \%$ of the patients in this study had experienced these side effects.

It has been reported that dementia occurs later in the course of disease and more rarely in younger patients (Giovannini, 1991; Quinn, 1987; Gibb, 1988; Lieberman, 1979; Ludin, 1989; Tanner, 1985a; Hietanen, 1988). In a populationbased cross-sectional investigation of PD in New York (Mayeux, 1992), dementia was not found in any patient with PD below the age of 50 years, but in $24 \%$ in the 60-69 year group, in 30\% in the 70-79 year group, and in 69\% of those older than 80 years. Disease duration between demented and non-demented patients was not different, suggesting that it is primarily age that influences the prevalence of dementia in PD. 
In this study, despite a median disease duration of 18 years, documented cognitive impairment had occurred in only $19 \%$ of patients. It occurred in only one patient $(5 \%)$ with a disease duration of less than 10 years, and in only $13 \%$ of patients below the age of 60 , whereas it was much more common above the age of $60(43 \%)$. It may be argued that any patient who might have demented would also be more likely to have died (Roos, 1996; Van Dijk, 1991), and hence the percentage of dementia in the deceased patients may have been higher. However, only two of the 12 patients who had been under our care until death had demented. Current age was the single most important predictive factor for dementia. At disease onset, female sex and a positive family history of parkinsonism were useful predictive factors for subsequent dementia and allowed prediction of dementia status in $67 \%$ of patients. However, the positive predictive value of these features was only around $30 \%$. This indicates that these features are relatively weak predictors in individual cases, and should be used with considerable caution. The reason for this increased risk of female patients to develop dementia is unclear as for once differential longevity is allowed for, there is no known increase of risk in women for dementia in general, or for Alzheimer's disease which may be an additional incidental pathology in many patients. The likelihood of developing dementia later on was further increased by a history of psychiatric side effects which may often indicate subclinical dementia (Kalra, 1996; Crystal, 1990). Although subtle changes on specific neuropsychological subtests may be more frequent in YOPD than in controls (Wermuth, 1996; Tsai, 1994), a low rate of dementia in YOPD was also found by other authors (Quinn, 1987; Tanner, 1985a; Mayeux, 1992) and contrasts with a cumulative incidence of up to $41 \%$ in the general PD population, which clearly exceeds the rate of dementia in an age-matched control population (Lieberman, 1979), despite a much longer disease duration in the patients in this series. A possible explanation for this dissociation between early occurrence of motor complications of therapy on the one hand, and late development of disabling dementia and axial symptoms on the other, may be a more restricted nigrostriatal dopaminergic deficit in YOPD. In contrast, in OOPD degeneration of other dopaminergic and other transmitter systems may be superimposed on age-related changes, including the presence of Alzheimer-type pathology (neurofibrillary tangles and senile plaques; Quinn, 1986; Hakim, 1979; Gaspar, 1984; Boller, 1980) and cerebrovascular disease, a combination of which may account for dementia and axial symptoms.

In 2 of the 56 patients (4\%) reported 10 years ago, the diagnosis was subsequently changed despite fulfilling the inclusion criteria at study entry. In the remaining 96\%, however, no new features indicating a disease other than Lewy body PD (LBPD) had evolved over a median disease duration of 23 (11-41) years. 
In two of these patients, and in five in the whole group who came to autopsy, LBPD was confirmed pathologically. The great majority of YOPD patients is therefore likely to suffer from LBPD. This view is supported by the few pathological reports in YOPD (Gibb, 1988; Yokochi, 1993). Gibb and Lees found no substantial difference in the pathology of patients with YOPD and OOPD but a greater nigral cell loss $(24 \%)$ in the YOPD group after a disease duration of 15 years compared to 3 years in the OOPD group.

$\mathrm{JP}$ is generally thought to be different from YOPD because of a higher occurrence of familial parkinsonism, a more common first presentation with dystonia, and varying pathology in the few autopsy reports (Van Bogaert, 1930; Davison, 1947; Yokochi, 1993) although this view has been questioned (Muthane, 1994). The small group of JP patients in this series comprised 10 subjects with differing clinical presentations. Dystonia was a presenting symptom in $50 \%$ of patients, which is significantly higher than in the YOPD group $(\mathrm{p}<0.05)$. Those five patients (50\%) all appeared to have a genetically determined disorder (2 probably autosomal dominant, 3 probably autosomal recessive), in one of whom this was combined with young-onset dementia. Genetic testing for abnormalities in the recently found Parkin gene interestingly revealed an abnormality in two of the familial and one of the seemingly sporadic cases. Clinically, the ten JP patients were also heterogeneous. This confirms previous reports of a high occurrence rate of familial parkinsonism and of disease heterogeneity in this group (Quinn, 1987; Yokochi, 1993; Muthane, 1994), only a minority of whom may have LBPD.

In conclusion, JP is a rare disorder probably representing several different diseases, whereas YOPD usually represents the lower end of the age spectrum of LBPD. The relative mortality is similar in YOPD and OOPD but life expectancy is reduced relative to an age-matched control population. Dyskinesias and motor fluctuations occur early and frequently, whereas cognitive function, postural reflexes and independence are preserved for many years provided the patients are still biologically and chronologically young. 


\section{Chapter 8. Quality of life in Parkinson's disease}

Parkinson's disease is a chronic progressive disorder for which there is no cure. It affects many aspects of patients' lives, only some of which can be appreciated by the available clinical rating scales. For instance, social embarrassment caused by a mild tremor is not appreciated by any of the clinical rating scales, and the isolation that can be the consequence of increasing disability is not reflected in these scales. In recent years patient evaluation has therefore shifted increasingly from traditional outcome measures, which focus only on the physician's perspective, to methods that incorporate the patients' perspective, which is the essence of health related quality of life measures. Subjective evaluation of quality of life (QoL) and health status is thus increasingly recognised to be important in the assessment and treatment of PD patients. Evidencebased evaluation of QoL has been made possible by the development of valid, reliable and sensitive instruments. Disease-specific instruments are valuable in assessing disease-specific problems and measuring success of treatment in altering QoL over time. Generic, i.e. non-specific, QoL measures, on the other hand, are designed to complement disease-specific QoL measures, and allow comparisons with other patient groups and the general population.

8.1. Comparison of the EQ-5D, the SF 36, two generic quality of life measures, and the PDQ 39, a disease-specific instrument, to measure quality of life in patients with Parkinson's disease

\subsubsection{Background}

Two instruments to measure quality of life in Parkinson's disease are now available. These disease-specific instruments are the PDQ-39, a disease-specific, well-validated, reliable and sensitive 39-item questionnaire on QoL in Parkinson's disease (Peto, 1995), and the PDQL, a validated, reliable and sensitive measure with 37 items (de Boer, 1996), which was developed recently and was not available at the beginning of this study. However, with these instruments, a comparison with the general population and other patient groups is not possible due to the disease-specificity of their questions ('Due to having Parkinson's disease, ... have you...?'). Generic instruments, which are 
not disease-specific, can therefore be valuable to supplement disease-specific QoL instruments to enable comparisons with other patient groups and to assess the impact of the disease on QoL in comparison to the general population. One such generic instrument, the widely used Medical Outcomes Study Short Form (SF 36) has been used in $\mathrm{PD}$ and been shown to have acceptable validity in this patient group (Rubenstein, 1998; Jenkinson, 1995). However, it is relatively long and has lower feasibility in an elderly patient group with a greater number of missing data (Brazier, 1996). The EQ-5D (EuroQoL Group, 1990; Brooks, 1996) is another generic QoL instrument, which has been extensively validated and been shown to be sensitive, internally consistent and reliable in the general population and other patient groups (Dorman, 1997; Hurst, 1994). Its usefulness in PD patients has not been examined. It is brief, comprising five questions and a visual analogue scale, which makes it easy to complete and attractive for use in an elderly population. In order to compare QoL in patients with $\mathrm{PD}$ with the general population and other patient groups, I examined the feasibility and validity of the EQ-5D instrument for assessing QoL in $\mathrm{PD}$ in comparison to the disease-specific PDQ-39 and the generic SF 36 in a population-based sample of patients with PD.

\subsubsection{Patients and methods}

All 124 patients with a diagnosis of probable PD in this population-based study on the prevalence of parkinsonism were included. All patients were asked to complete the disease-specific PDQ-39 (Peto, 1995), and the generic EQ-5D (EuroQoL Group, 1990) and SF-36 (Brazier, 1992), and to return them in a stamped, addressed envelope. 8.1.2.1. Qol instruments: The EQ-5D (EuroQoL Group, 1990) is a short questionnaire with 5 questions on mobility, self-care, pain, social activities and psychological status and 3 response options ( $1=$ no problem, $2=$ moderate and $3=$ extreme problem). A summary index with a maximum score of one can be derived from these 5 dimensions (maximum score 1.0 indicating best possible health state). In addition, there is a visual analogue scale to indicate general health status (100 indicating best health status). The Medical Outcome Study Short Form (SF-36; Brazier, 1992) comprises 36 questions with 2 to 5 response possibilities. Eight different subdimensions and a physical and mental summary score can be derived from these (maximum score 100 indicating best 
possible health state). Both have been shown to be valid, reliable and sensitive in the general population and in a variety of disorders (Jenkinson, 1993; Hurst, 1994). They were also found to be valid and feasible in this population-based sample of PD (table 32). The PDQ-39 is a disease-specific instrument, which has been shown to be feasible, reliable, valid and responsive to change in patients with PD (Peto, 1995). It comprises 39 questions with 5 different response options, from which 8 subdimension scores and one summary index can be calculated (maximum score 100 indicating worst level of problem).

Feasibility was determined by the number of missing responses in each instrument. The validity of an instrument is the extent to which it measures what it claims to measure. Different types of validity can be assessed. The concurrent validity of the EQ-5D was evaluated by studying the relationship between the scores on this scale and other generic and disease-specific measures of QoL as well as parameters reflecting disease severity. Convergent validity was determined by correlating with other QoL instruments, and discriminant validity by comparing groups of patients with and without particular clinical features (Anastasi, 1976).

8.1.2.2. Statistical analysis: The subdimensions and summary scores were calculated according to the respective scoring algorithms. Nonparametric tests for comparisons were selected for the analysis of QoL scores because of the ordinal nature of the scales. Group differences were analysed by the Mann-Whitney test and associations between scales with Spearman rank correlations. In order to take into account the multiple number of comparisons performed, a statistical threshold of $\mathrm{p}<0.005$ was considered to be sufficiently conservative. Differences with $\mathrm{p}<0.01$ and $\mathrm{p}<0.05$ were considered as marginally significant.

\subsubsection{Results}

Five questionnaires were returned with incomplete data (less than $50 \%$ of the QoL instrument), and were not used for further analysis. There was no statistical difference between responders and non-responders with respect to age, disease duration, sex, Hoehn and Yahr stage and Schwab and England score. The characteristics of the responders are given in table 32 .

\subsubsection{Feasibility}


97 of the 124 patients returned the questionnaires, which constitutes a response rate of $78 \%$. Their mean age was 73 (SD 11.3, ranging from 36 to 94 ) years with a mean disease duration of 5.8 (SD 4.9, ranging from 0.2 to 25) years, and a mean onset age of 67.6 (SD 12.5, ranging from 23.7 to 93.5 ) years. There were 50 men and 47 women who responded, and mean Hoehn and Yahr stage was 2.4 (SD 0.9, ranging from 1 to 5). There were 20 (4.1\%) missing responses on the EQ-5D and $6(6.2 \%)$ patients had not completed the VAS. On the PDQ-39, 180 answers were missing (4.8\%), and on the SF36286 answers $(8.2 \%)$ were not completed.

\subsubsection{Validity}

The validity of the EQ-5D was examined in several ways. First, by measuring the association between the EQ-5D summary index, the subscores, and the visual analogue score with the clinical ratings of disease severity in PD (table 33). Second, by examining its correlation with the generic (SF 36) and the disease-specific (PDQ 39) measures of QoL (table 34). Third, by determining whether the EQ-5D successfully differentiated between patients with and without features of advanced disease, such as those with or without falls or dementia (table 35).

\subsection{Correlation with ratings of disease severity (table 33)}

\subsection{EQ-5D summary index}

As expected, lower EQ-5D summary index scores correlated significantly with greater disease severity as measured by Hoehn and Yahr stages $(r=-0.68, p<0.001)$ and with lower independence scores, as measured with the Schwab and England disability scale $(r=0.66, p<0.001$; table 33). Specific dimensions of QoL, as measured in the subscores, also correlated significantly with both disease scales but, as expected, in the opposite direction. Higher scores on the motor section of the UPDRS correlated significantly with lower EQ-5D summary scores $(r=-0.40, p<0.001)$ and with a higher degree of impairment in the subscores of mobility and pain $(r=0.40, \mathrm{p}<0.001$, and $\mathrm{r}=0.31$, $\mathrm{p}<0.001$ ). Low MMSE scores showed the highest significant correlations with greater degree of impairment on the mobility and usual activities subscores (both $\mathrm{r}=-0.37$, $\mathrm{p}<0.001$ ). Disease duration correlated most with a greater degree of impairment on the mobility subscore of the EQ-5D $(r=0.35, \mathrm{p}<0.001)$.

\subsection{Visual analogue scale}


The correlation of the VAS with measures of disease severity were in the same direction but less strong than those obtained for the EQ-5D summary index. The VAS correlated significantly with the degree of independence quantified with the Schwab and England scale $(r=0.42, \mathrm{p}<0.001)$ and negatively with the depression score as measured by the BDI $(r=-0.63, p<0.001)$, but not with the motor section of the UPDRS, and only marginally significantly and negatively with the Hoehn and Yahr scale ( $r=-$ $0.32, \mathrm{p}<0.01)$.

\subsection{Correlation with other QoL scales (table 34)}

The EQ-5D summary index correlated strongly and negatively with the PDQ-39 summary index $(\mathrm{r}=-0.75, \mathrm{p}<0.001)$ and most PDQ-39 subscores, and positively with the SF 36 scores except for the 'Emotional Role' subscore (see table 34). As expected, the strongest associations were between the EQ-5D and PDQ-39 or SF 36 subscores of both instruments that measure similar aspects of QoL, indicating good convergent validity. The strongest, negative, correlation was found between the EQ-5D and the PDQ-39 summary indices $(r=-0.75, \mathrm{p}<0.001)$. The VAS of the EQ-5D correlated significantly with all the SF 36 (positively) and PDQ-39 scores (negatively) with correlations ranging from 0.40 to 0.62 , except the 'Social Functioning' subscore of the PDQ-39, and the 'Emotional Role' subscore of the SF 36, which were less strongly and only marginally significantly correlated with the VAS $(r=-0.30, p<0.01$, and $r=0.25$, $\mathrm{p}<0.05$, respectively).

\subsection{Sensitivity to clinical features of Parkinson's disease}

\subsection{EQ-5D summary index and Visual analogue scale}

Using a cut-off score of 14 to define presence or absence of moderate or severe selfreported depression as measured on the $\mathrm{BDI}$, the EQ-5D summary index was signficantly different for patients with and without depression as measured on the BDI $(p<0.0001$; Beck, 1961). Patients with cognitive deficits as indicated a by a MMSE less than 25 had significantly lower EQ-5D scores than the group without cognitive deficits $(p<0.0001)$. The features of the illness which had a significant impact on EQ-5D scores were presence/absence of falls $(p<0.0001)$, postural instability $(p<0.0001)$, on-off fluctuations experience of hallucinations (the latter two only marginally significant, $p=0.046$ and $p=0.007$, respectively). In contrast, age (being older or younger than 70 years) had no significant impact on EQ-5D scores. In addition, patients who felt that 
their general health had deteriorated over the past year had significantly worse EQ-5D scores than those whose health had subjectively improved or remained much the same $(p<0.0001)$. There was no significant difference in EQ-5D scores between those patients who felt improved compared to one year before and those who had deteriorated, but this may have been due to the small number of patients $(n=6)$ who had noticed an improvement.

The visual analogue scale was less sensitive to these disease-related features and only significantly differentiated groups with or without depression $(\mathrm{p}<0.0001)$, postural instability $(p<0.0001)$, deterioration of general health over the past year $(p<0.0001)$, falls $(\mathrm{p}<0.001)$ and cognitive deficits as well as older patients (both only marginally significant $0<0.007$ and $\mathrm{p}<0.013$; see table 35).

\subsection{Comparisons with other QoL measures}

The correlation of the EQ-5D summary index and its subscores with the clinical scales was very similar to that of the PDQ-39 and the SF 36 summary scores (table 33). The correlation of the VAS with these scales, on the other hand, was weaker than that of the PDQ-39 summary index and SF 36 physical summary, except its correlation with the BDI. However, the association of the VAS of the EQ-5D with the clinical measures was better than the correlation of the mental summary of the SF 36 with these scales. Furthermore, the EQ-5D significantly differentiated between patients with and without particular clinical features such as falls, postural instability, worsening of health over the past year, on-off fluctuations, hallucinations, cognitive deficits or depression as well as the PDQ-39 and better than the SF-36 physical score (see table 35).

\subsubsection{Discussion}

The EQ-5D summary index had good feasibility and validity in this population-based sample of patients with PD. It correlated moderately with the measures of disease severity and disability as well as with depression and cognitive impairment, reflecting its sensitivity to disease severity, depression and cognitive deficit coupled with sufficient distinctiveness. It also correlated strongly with the disease-specific PDQ-39 summary index and its subscores and, to a lesser degree, with the physical score of the generic health-status instrument SF-36. Its sensitivity to the clinical features of 
Parkinson's disease was similar to that of the disease-specific instrument, whereas the SF-36 was less sensitive.

These results are similar to those reported for the disease specific QoL instrument, the PDQ-39 (Peto, 1995). The validity of the EQ-5D summary index in this sample of patients with $\mathrm{PD}$ was high and better than reported for other disorders such as epilepsy (Selai, 1998) or lower limb ischemia (Chetter, 1997) and comparable to that of the disease-specific PDQ-39. A possible explanation for this finding could be that the correlation of the PDQ-39 with clinical scales was poorer than in previous studies, which were either clinic-based or used a selected sample (members of the PD society; Peto, 1995). However, the correlation of the PDQ-39 and the SF 36 with the Hoehn and Yahr and other clinical scales found in this study was very similar to previously reported results (Fitzpatrick, 1997; Rubenstein, 1998) confirming their validity in an unselected sample of patients with PD. Therefore, at first sight it may appear surprising that a non-specific generic measure which only comprises five questions with three levels of response option correlated well with the clinical scales. However, it should be noted that these five questions cover five core areas which are particularly important in PD: mobility, self-care, usual activities, pain, and anxiety/depression. They represent the aspects of life most affected by PD and its typical features. Other features like stigma, social support, communication and cognition are not covered, but the areas most relevant for QoL in PD, as found by Fitzpatrick et al (Fitzpatrick, 1997), are also those covered in the EQ-5D: difficulty with leisure activities, difficulty looking after the home, problems writing clearly (covered in 'usual activities'), difficulties getting around in public places, problem walking half a mile, difficulty carrying shopping bags (covered in 'mobility'), problems in doing up buttons or shoelaces (covered in 'selfcare'), worrying about the future (covered in anxiety/depression) and bodily aches and pains (covered in 'pain'). The EQ-5D may be a particularly suitable generic instrument for patients with $\mathrm{PD}$ as it is a very mobility-based questionnaire. It may also be more valid in this patient group than for example in patients with epilepsy or headache as there are no disease-free intervals which could distort the true extent of the problem when asked for the 'health state today'. It may also be more suitable for patients with PD than for patients with congenital or early onset disorders as PD usually starts late in life so that patients can easily compare their current to their previous health state. The 
VAS part of the EQ-5D, although easy to complete, was not as strongly correlated with clinical measures or sensitive to the presence or absence of clinical features. On the other hand, it correlated highly with depression.

The SF-36 was a generally less sensitive measure (lower correlation with clinical scales, and poorer differentiation between patients with and without features of advanced disease) than the EQ-5D summary index and the PDQ-39, and additionally had a higher percentage of missing answers. Moreover, patients frequently added comments to its questions as they did not feel these were appropriately reflecting their problems, such as 'I have arthritis and can therefore not walk long distances', or 'I am widowed' on the question on a decrease of libido.

A shortcoming of this study is that the EQ-5D's sensitivity to change was not formally assessed, which is important if a QoL instrument is to be used in clinical trials to test effectiveness of treatment. This was a cross-sectional study with only one evaluation of QoL. However, patients who reported deterioration of their general health over the past year had significantly worse QoL scores than those whose health had not deteriorated or improved, indicating the instrument's ability to reflect change in disease severity.

The EQ-5D appears to be feasible and valid in a population-based sample of patients with $\mathrm{PD}$, and comparable to disease specific instruments. Its sensitivity to change needs to be assessed in further studies. 


\subsection{The impact of Parkinson's disease on quality of life}

\subsubsection{Background}

Most previous studies of QoL in PD have assessed selected samples of patients derived from specialist clinics (Fitzpatrick, 1997; Rubenstein, 1998) or members of the PD society (Fitzpatrick, 1997). To date, only one study in Norway has assessed QoL in a population-based sample of patients with PD using the generic Nottingham Health Profile (NHP; Karlsen, 1999). However, no comparisons with the general population were made and, to my knowledge, normative data for the NHP from the Swedish general population are not available.

In order to determine the impact of Parkinson's disease on QoL, I assessed QoL, as measured by the disease-specific and the two generic instruments, in this populationbased sample of patients with PD in London, and compared it to the published results in the general UK population.

\subsubsection{Patients and methods}

All 97 patients with PD who had returned the questionnaires were included.

Statistical analysis. Mean values were compared by Mann-Whitney and Kruskal-Wallis tests, and Spearman rank correlations. Categorical data were analysed using the Chisquare test. Statistical significance was accepted at the $\mathrm{p}<0.005$ level.

Published normative values of the SF 36 and the EQ-5D in the UK population, which were taken from studies providing normative values in the relevant age groups (Brazier, 1992; Kind, 1998), were used as comparative data. Data from another large survey providing population norms for the SF 36 separately for men and women could not be used due to small numbers in the respective age-groups for men and women in this study.

\subsubsection{Results}

\subsubsection{The EQ-5D instrument}

A moderate or severe problem in at least one dimension was reported by $79(81.4 \%)$ patients (moderate in $77 \%$, and severe in $27 \%$; not mutually exclusive; figure 8 ). The most frequently reported problems were in the domain of mobility followed by social 
functioning and pain. Compared to the general population, PD patients reported problems more frequently in all dimensions at all ages, apart from the age group of 4049 , as only one patient with PD was in this young age group. Compared to norms available from the general population (Kind, 1998) the most striking differences were seen in the domains of self-care, social functioning, mobility, and anxiety/depression, with 2 to 10 times more patients reporting problems than the general population. In contrast, pain and discomfort occurred also in a substantial proportion of the general population. The differences were generally most marked in the younger age groups (excluding age group 30-39 and 40-49 as they only comprised 3 patients), and diminished with increasing age. Only anxiety/depression had a markedly higher prevalence in the oldest age group of $\mathrm{PD}$ patients compared to the general population (table 36).

The mean state of health recorded by PD patients on the visual analogue scale was 64.0 (SD 22.6). The mean score differed from that of the general population for all age groups (figure 9).

There were no significant differences in the percentage of patients indicating a problem between men and women with PD, in different age groups or social classes, even if controlled for Hoehn and Yahr stage. There was however a trend for female patients, patients over 70 years old and patients in lower social classes to have a worse perception of their general health as measured by the VAS ( $p<0.05$ for all three comparisons). The EQ-5D summary index was significantly different for different stage of illness as measured by the Hoehn and Yahr scale $(p<0.001$; table 37). This effect of stage of illness was seen in all EQ-5D subdimensions, but there was a ceiling effect for the mobility and usual activity subscores, as problems in these areas were already reported by all patients at stage III (figure 10). The EQ-5D summary score correlated positively with disease duration $(\mathrm{r}=0.35, \mathrm{p}=0.001)$.

\subsubsection{The Medical Outcome Study Short Form (SF 36) instrument}

Lower scores on the SF 36 reflect poorer health status. A comparison of SF 36 scores with the general UK population (Brazier, 1992) showed that PD patients had poorer SF 36 scores than the general population in the same age group (table 38). The greatest differences from the general population norms were seen in the domains of physical 
and social functioning, physical role limitations and general health perceptions, particularly in the younger age groups. In contrast, pain, emotional role limitations, vitality and mental health were only slightly worse for the PD patients than in the agematched UK sample.

The physical subscore correlated with age $(\mathrm{r}=0.33, \mathrm{p}=0.001)$, and there was a trend for patients in lower socioeconomic groups to have worse subscores of mental health and vitality (both $\mathrm{p}<0.05$ ), but otherwise there was no significant difference between SF 36 scores in men and women with $\mathrm{PD}$, or between age groups or social classes. The physical functioning and social functioning subscores of the SF 36 showed a significant effect of Hoehn and Yahr stage of illness ( $p<0.001$ for both subscores) and there was a trend for worse subscores of physical role limitation, general health perceptions, vitality, mental health and pain in higher disease stages $(p<0.01$; figure 11$)$. There was no correlation between any of the SF 36 subscores and disease duration.

\subsubsection{The $P D Q-39$ instrument.}

The maximum score of this disease-specific instrument is 100 , which indicates the poorest possible health status. The scores obtained in this population-based sample of patients with PD was similar to those reported in a clinic-based sample of patients with PD (Fitzpatrick, 1997), but better than those reported in a population-based sample of members of the PD society (Fitzpatrick, 1997; table 39). All subscores apart from social support and stigma reflected significant effects of disease stage $(p<0.001$; figure 12). There was no difference in PDQ-39 scores between men and women. The only subscore that correlated significantly with age was mobility $(r=0.33, p=0.002)$. Only the activities of daily living subscore correlated marginally significantly with disease duration $(\mathrm{r}=0.31, \mathrm{p}=0.007)$.

\subsubsection{Discussion}

In this representative cross-sectional study of patients with $\mathrm{PD}, \mathrm{QoL}$ and subjectively evaluated health status were impaired at all ages and in both sexes. QoL, as measured by all three instruments, deteriorated significantly with increasing stages of illness. The mean index score of the EQ-5D in patients with the worst disease severity (Hoehn and 
Yahr stage V) even reached a negative value, which is considered lower than the worst possible health state in a subjective health state evaluation (table 37). The spheres of daily life which significantly deteriorated with advancing disease were predominantly those associated with mobility, self-care, activities of daily living, physical and social functioning, but also cognition, communication, bodily discomfort and (on the diseasespecific instrument only) emotional well-being. Stigma and social support did not deteriorate significantly with advancing disease. The social support dimension of the PDQ 39 measures predominantly close ties and family support rather than distant relationships. Fitzpatrick et al also found that social support as measured by the PDQ 39 is not greatly influenced by disease severity in patients with $\mathrm{PD}$, and other diseases (Fitzpatrick, 1997; Fitzpatrick, 1988). This suggests that close relationships are less jeopardised by chronic disease than distant relationships. Age correlated with the mobility subscore of the PDQ-39, but otherwise neither age nor gender had a significant impact on QoL in PD despite the fact that being older, being female, and belonging to lower socioeconomic groups are associated with poorer QoL in the general population (Jenkinson, 1993).

Compared to the UK general population, this group of PD patients had worse QoL scores in all areas, apart from pain, which has been reported to have a high prevalence in the general population (Kind, 1998), and was only slightly more common in PD patients. The greatest difference between the general population and the PD sample was seen in the area of self-care, where PD patients reported problems up to 10 times more frequently. Other areas related to physical function were also significantly impaired in PD. The difference in the percentage of patients reporting problems appeared greatest in the younger age groups, particularly in the subdimensions of the EQ-5D instrument, although no conclusions can be drawn from those below 50 due to small numbers. Younger people generally lead more active lives than older people do, with higher expectations and responsibilities. Therefore, the interference of the symptoms of PD with their everyday life is likely to be greater than in older people. Only in the domain of anxiety/depression was there a difference between PD patients and the general population in the oldest age group, which may relate to increasing loneliness and isolation. However, the larger difference of QoL between the general population and PD patients in younger age groups was not seen in the visual analogue 
scale of the EQ-5D, which measures the patients' overall assessment of their general health status.

It was not possible to directly compare the results in the PD group with those in the general population statistically as there was no general population-based control group in this study. However, the studies providing norms for comparable age groups of the UK population were considered representative of the UK population (Kind, 1998; Brazier, 1992), and this sample was based on a population-based prevalence study. The samples within the respective age groups are therefore likely to be comparable.

To my knowledge, this is the first study providing comprehensive QoL and subjective health state evaluations in patients with PD in comparison to the general population in the UK. However, health-related QoL as measured by the Nottingham Health Profile (NHP) was reported recently in a sample of patients with PD participating in a prevalence study in Norway (Karlsen, 1999). The results are broadly similar to the findings in this study. Patients with PD had worse QoL scores in all dimensions than the general population, and there was no difference between men and women. The worst scores were found in the area of physical mobility, but sleep and energy also revealed high rates of impairment. Fitzpatrick et al (1997) used the disease-specific PDQ-39 and the SF 36 in a clinic-based sample of patients with PD and reported scores in the same range as in this study. However, the results from a population-based sample (members of the PD society) reported by the same group were consistently worse. This may be explained by truly poorer QoL in a population-based sample of patients with $\mathrm{PD}$, who may have generally less access to specialist treatment, as the authors suggested. However, the patients were not examined prior to inclusion in the study, but were recruited on the basis of their membership in the PD society. It is therefore difficult to establish what disease stage these patients were or how accurate the diagnosis was. Therefore, patients with other disorders like multiple system atrophy, progressive supranuclear palsy as well as those with vascular parkinsonism may have been inadvertently included in their population-based sample. Furthermore, populationbased samples of active members of the PD society are not necessarily representative of the general population, but are a selection of patients who actively seek support because of their disease. In contrast, in one study in Northern America (Rubenstein, 1998), which used the SF 36 in a clinic-based sample of patients diagnosed with PD, 
the QoL scores were slightly better across all ages in all dimensions relative to this study (table 39). In that study fewer patients had advanced disease, which is likely to explain this difference. An important advantage of the present study is that it was not based on a selected sample of patients (clinic-based or members of the PD society) but on a population-based prevalence study with high participation rate (84\%). In addition, the diagnosis is likely to be more accurate as each patient was examined and strict clinical diagnostic criteria applied, since the primary purpose was the differentiation of different parkinsonian syndromes.

In summary, the main impairment of QoL in PD lies in the areas related to mobility and physical functioning, and increases with increasing disease severity. Younger patients appeared to have worse QoL compared to the age-matched population, but there was no difference in QoL scores between men and women. Efforts to improve health care for patients with $\mathrm{PD}$ should be designed to consider these aspects of QoL in patients with PD. 


\subsection{Factors that contribute to quality of life in patients with Parkinson's disease}

\subsubsection{Background}

Treatment of $\mathrm{PD}$ is primarily aimed at improving motor function. However, especially in advanced stages, PD is often complicated by additional problems such as treatment-related complications, falls, depression, and dementia (Lang, 1998), which may have much greater impact on the patients' quality of life (QoL) than the cardinal features of PD. A disease-specific QoL instrument for use with patients with PD, the PDQ-39, has been shown to have good reliability, validity, responsiveness and reproducibility (Peto, 1998, Fitzpatrick, 1997a, Peto, 1995), and is now used in many treatment trials in PD to assess effectiveness of treatment (Fitzpatrick, 1997b). Its disease specificity and the single summary index offer the opportunity to assess the overall impact of illness, and it is easy to interpret (Jenkinson, 1997). In this study the PDQ 39 was used to determine which diseaserelated factors, such as degree of impairment, presence of specific symptoms, disability, depression and cognitive impairment, are the most relevant for quality of life in this population-based sample of patients with PD.

\subsubsection{Patients and methods}

All 124 patients with probable PD were included. Patients with atypical parkinsonian disorders, drug-induced parkinsonism, vascular pseudoparkinsonism and those with parkinsonism following dementia were excluded.

Statistical analysis. Stepwise multiple regression analysis was used to determine the factors that best accounted for variance in QoL scores. Due to the number of different comparisons, statistical significance was only accepted at $\mathrm{p}<0.005$. Differences with $\mathrm{p}<0.05$ were considered as marginally significant.

\subsubsection{Results}

\subsubsection{Impact of clinical features on $P D Q-39$ scores}

Patients with high levels of depression (with BDI scores of 18 or more), a minimental state score of 24 or less (out of 30 ), postural instability on examination (as described in the UPDRS), a history of falls or of gait difficulties had significantly worse PDQ-39 summary index scores than patients without these features. QoL 
scores of patients with the akinetic-rigid subtype of PD were also worse than of those with tremor-dominant PD. This difference could not be explained by older age or longer disease duration, as age was similar between those with tremordominant and those with akinetic-rigid Parkinson's disease, and the difference in disease duration just failed to reach statistical significance. The difference of QoL scores between those with and those without a history of hallucinations was marginally significant (table 35). No difference in PDQ-39 scores were found between males and females and between patients with or without a poor (subjectively $<50 \%$ ) initial or current response to treatment with antiparkinsonian medication. A history of dyskinesias or fluctuations, incontinence, orthostatic symptoms, insomnia, pain, speech or swallowing impairment, a family history of $\mathrm{PD}$, and symptom at onset had no significant impact on QoL scores. There was also no difference between those who were unemployed or had retired early due to the disease and those who were not, those with disease onset before or after age 50, and those with current age above or below 60 or 70 .

\subsubsection{Correlations of clinical scores with $P D Q-39$}

The PDQ-39 summary index correlated significantly and strongly $(r=0.68, p<0.001)$ with depression as measured by the BDI score and negatively with disability as measured by the Schwab and England scale $(r=-0.66, p<0.001)$. Significant correlations were also obtained with disease severity as measured by the Hoehn and Yahr scale $(r=0.6, p<0.001)$, the UPDRS motor subscore of axial features $(r=0.57$, $\mathrm{p}<0.001)$, the total UPDRS motor score $(\mathrm{r}=0.41, \mathrm{p}<0.001)$ and the MMS $(\mathrm{r}=-0.32$, $\mathrm{p}<0.001)$. Akinesia score $(r=0.3, p=0.028)$ and socioeconomic groups $(r=0.25$, $\mathrm{p}=0.023$ ) were marginally significantly correlated to QoL, whereas correlations with disease duration and age were not significant $(r=0.18$ and $r=0.14$, respectively).

\subsubsection{Regression analysis of $P D Q 39$ scores}

In order to determine which factors contributed most to QoL scores, a stepwise linear regression was performed, entering age, disease duration and all factors that were shown to have an impact on QoL scores into the regression in a stepwise fashion (table 40). The most important predictive factor was the BDI depression score, followed by disability as measured by the Schwab and England score, which together accounted for $64 \%$ of the variance of QoL scores (table 40). Additional 
contributing factors were postural instability and cognitive score, which accounted for a further $8 \%$ on the variance of QoL scores. In order to account for some of the overlap of contents in quality of life and disability measures, as well as the colinearity of Schwab and England scores with Hoehn and Yahr scores ( $\mathrm{r}=-0.74$, $\mathrm{p}<0.0001$ ), a separate analysis excluding Schwab and England scores from the analysis was performed. Depression scores, Hoehn and Yahr scores and cognitive score together still accounted for $59 \%$ of the variance of quality of life scores.

\subsubsection{Discussion}

In this study, the strongest predictor of QoL in PD was the presence of depression, as measured by the Beck Depression Inventory score. This correlation of QoL with depression has also been found in other studies on QoL in PD (Martinez-Martin), but also in other chronic diseases where depression has been found to have a major influence on QoL scores (King). It could be argued that the PDQ 39 and the BDI measure the same content and hence have a strong correlation. However, the PDQ 39 comprises 8 different dimensions of which depression is a feature in only one. In addition, the results were similar when two other, generic, QoL instruments were used. The association of PD with depression is well known, but treatment of depression, in PD as well as the general population, is frequently insufficient (Livingstone). The results of this study highlight the need to diagnose and address this feature in PD more effectively in order to improve the patients' QoL.

As may be expected, QoL was also strongly related to Schwab and England disability scores. However, there is some overlap in the content of QoL and disability scales, and, additionally, disability scores also strongly correlated with disease severity, as measured by the Hoehn and Yahr scale $(r=-0.74, p<0.0001)$. In order to reduce these problems of overlap of disability scores with QoL on the one hand, and collinearity with disease severity on the other hand, a separate regression analysis was performed excluding disability as a variable. In this analysis, the results were similar, but, as might be expected, disease severity replaced disability. In addition, postural instability, which is one of the key criteria for staging in the Hoehn and Yahr scale and was therefore associated with higher Hoehn and Yahr scores $(\mathrm{p}<0.005)$, no longer additionally contributed to predicting QoL scores. Cognitive scores, on the other hand, continued to contribute further to the prediction of QoL scores, as they were not strongly associated with depression ( $\mathrm{r}=-0.15, \mathrm{~ns})$ or 
Hoehn and Yahr scores $(r=-0.27, p<0.05)$. As cognitive impairment does not respond to, and can be aggravated by, medication that improves mobility, it presents a major problem in the management of $\mathrm{PD}$, and has been shown to be the primary reason for nursing home placement (Goetz, 1993).

The correlation of QoL scores with disease severity and disability, which has been shown previously (Pet, 1995; Peto, 1998; Karlsen, 1999), is not surprising. However, the motor part of the UPDRS, which is the primary outcome measure for most treatment trials in PD, correlated less strongly with QoL scores. This scale, in contrast to the Hoehn and Yahr scale, is a detailed assessment of parkinsonian features, comprising items of speech, hypomimia, tremor and rigidity in addition to bradykinesia and axial features such as postural instability and gait. However, these subscores did not have a significant influence on QoL scores. As a consequence, the Hoehn and Yahr scale, which reflects predominantly axial features, correlated more strongly with QoL scores than the motor part of the UPDRS.

Tremor and rigidity scores did not correlate with QoL scores. Gait problems and limb akinesia, on the other hand, were associated with worse QoL although not contributing further to its prediction once disease severity was accounted for. Similarly, Peto et al (Peto, 1995) found that self-reported slowness was significantly related to 7 out of the 8 subdimensions of the PDQ-39, whereas self-reported tremor and rigidity were much less markedly correlated with the PDQ-39 subdimensions.

Patients with the akinetic-rigid subtype of PD (which incorporates impairment of axial features) had worse QoL scores than those with the tremordominant subtype of PD. In studies assessing sub-types of parkinsonism, tremordominant PD has usually been found to have a better prognosis than the akineticrigid subtype of $\mathrm{PD}$, with a slower rate of progression of motor symptoms (Zetusky, 1985; Jankovic, 1990). The findings of a better QoL in tremor-dominant PD in this study further support this differentiation. Symptoms at onset, on the other hand, did not significantly affect QoL scores. However, information on type of symptom at onset was collected by patient report, which has a low reliability (Richards, 1994).

A history of hallucinations on antiparkinsonian treatment was also marginally significantly associated with worse QoL scores, although, in contrast to previous reports (Sanchez-Ramos, 1996), it was not significantly associated with worse cognitive scores. This finding illustrates the importance of striking a balance between benefits and side effects of medication in the treatment of PD, and is in 
agreement with reports that the presence of hallucinations predicts nursing home placement (Goetz, 1995).

Surprisingly, a history of fluctuations of motor response to medication or presence of dyskinesias was not associated with poor QoL. This may be due to the relatively small number of patients in this population-based sample with these complications, which, if present, were usually not severe.

In accordance with the findings in this study, Karlsen et al (Karlsen, 1999) also found that depression and disability are important predictors of QoL in PD. However, in contrast to this study, postural instability did not correlate as strongly with QoL, whereas insomnia, which did not predict QoL in this study, was an important influential factor in that study. This difference is likely to arise from their use of the Nottingham Health Profile (NHP), which is a generic instrument to measure health status. It is not a disease-specific instrument, and does not therefore address specific problems that arise in PD. It has been used to assess QoL in patients with PD in a large study of Sinemet CR (Block, 1997), but its validity in patients with $\mathrm{PD}$ has not been formally tested. In addition, it is highly skewed at the severe end of ill health, and will not detect subtle impairments. On the other hand, the section on sleep is larger in the NHP than in the disease specific PDQ-39, and insomnia is therefore more likely to correlate with NHP scores.

QoL is a complex concept to which many factors other than health contribute. This complexity of the concept of QoL is also the main limitation of this study, as many parameters that potentially contribute to QoL, such as social support and individual coping strategies, were not directly measured. Also, I have no information on variables such as the cultural context of the patients' lives or availability of health care resources, which may have great potential for modifying the impact of the disease on the QoL in PD.

On the positive side, the results of this study are likely to be generalisable to a wider population of patients with $\mathrm{PD}$, as the patients were seen in a populationbased epidemiological study with a high response rate (84\%), and that the results are clinically relevant in this patient group due the disease-specificity of the instrument used.

In conclusion, QoL in IPD was primarily determined by depression, disability, disease severity (particularly related to axial features), and cognitive impairment. Patients with the akinetic-rigid subtype of PD had worse QoL scores 
than those with tremor-dominant PD. The motor part of the UPDRS, although the primary outcome measure for clinical trials with antiparkinsonian medication, was not the determining measure for QoL in Parkinson's disease, and additional emphasis on other features should therefore gain greater importance in the treatment of PD. 


\section{Chapter 9. Summary, conclusions, and implications}

In this thesis I investigated the prevalence and incidence of different typical and atypical parkinsonian syndromes and their subtypes in the general population. In addition, this representative sample of patients with PD served to determine the existence of different subtypes within this heterogeneous disease. I also endeavoured to analyse the rate of motor complications and depression in the patients with $\mathrm{PD}$, and to determine the factors that predict the occurrence of these complications. Finally, subjectively evaluated quality of life in this population-based sample of patients with $\mathrm{PD}$ was assessed and compared with that in the general population, the factors that contribute to it most were determined, and the validity of different instruments to measure QoL in these patients were examined.

The findings of the studies were discussed in detail at the end of each Chapter. The purpose of this final section is to briefly summarise the findings of the studies, in order to draw conclusions, and give directions for future research and management of patients with parkinsonian disorders.

\subsection{The population prevalence and incidence of atypical parkinsonian disorders}

The prevalence of PSP and MSA in the general population was previously unknown. Clinico-pathological studies of patients diagnosed as having PD suggested that both disorders are frequently underdiagnosed, but these series were likely to be biased towards an over-representation of such cases. In this population-based study with a participation rate of $84 \%$, the age-adjusted prevalence rate for PSP was determined as 6.4 per 100,000 , based on 5 probable and 1 possible cases, with $95 \%$ confidence intervals of 2.3-10.6 per 100,000. The age-adjusted prevalence rate for MSA was 4.4 per 100,000 , based on 2 probable and 2 possible cases, with $95 \%$ confidence interval 1.2-7.6 per 100,000. An additional 4 atypical patients were ascertained but did not fulfil published criteria for PSP or MSA. These prevalence rates were lower when only patients who fulfilled the clinical criteria for probable MSA or PSP are included, but higher if the patients with atypical features, who did not fulfil the criteria for possible MSA or PSP, are included. 
Interpretation and implications: These results suggest that prevalence studies of PSP and MSA, which do not examine all cases of parkinsonism regardless of diagnosis, will underestimate the true prevalence rate as many patients in the community remain misdiagnosed.

\subsection{The population prevalence and incidence of Parkinson's disease}

The prevalence rates for IPD and parkinsonism, adjusted to the English population, were 168 (95\% confidence interval 142 to 195) and 254 (95\% confidence interval 222 to 287 ) per 100,000 , respectively. Taking into account an estimated $10-20 \%$ of patients not having come to medical attention, the prevalence of IPD in London is estimated as approximately 200 per 100,000. In both sexes the rate of IPD and parkinsonism increased with age and peaked at $1.3 \%$ and $1.8 \%$, respectively, in the oldest age group ( $>80$ years). IPD accounted for $66 \%$ of all parkinsonian syndromes, atypical primary parkinsonism for $6 \%$, drug-induced parkinsonism for $18 \%$, parkinsonism following dementia for $2 \%$ and vascular pseudoparkinsonism for $7 \%$.

Interpretation and implications: The prevalence of PD in London is similar to that in other parts of the United Kingdom, and comparable to that in most other parts of the world. Assuming that $10-20 \%$ of patients had escaped medical attention, the prevalence of PD in London is estimated as approximately 200 per 100,000. Prevalence rates increase with advancing age without a decline in the oldest age group, indicating that a previously found decline of prevalence in the oldest age group was likely to be due to underascertainment. However, the total number of patients with parkinsonism, including atypical parkinsonism, DIP, vascular parkinsonism, and parkinsonism following dementia, is considerably higher. Due to the differences in aetiology, prognosis, treatment and needs of patients, these disorders should be differentiated from patients with PD in future clinical and epidemiological studies and calculations for the provision of health care resources in the population.

\subsection{Accuracy of the clinical diagnosis in Parkinson's disease in the community}

As shown in pathological studies, many patients diagnosed with PD in fact have a different disorder, and, conversely, not all patients with PD in the population may be 
recognised to have it. This study assessed the accuracy of a clinical diagnosis of PD according to strict published criteria in the general population. A diagnosis of probable PD was confirmed in $83 \%$ of patients with this diagnosis, including three patients $(2 \%)$ in whom atypical features were found, but were not sufficient to discard the diagnosis of PD. Two additional patients (2\%) were found to have possible PD. However, in $15 \%$ of patients the diagnosis of PD was unequivocally rejected. Conversely, 15 patients $(19 \%)$ who had previously not been diagnosed as $\mathrm{PD}$, were found to have this disorder. Conclusions and implications: If strict clinical criteria are used, at least $15 \%$ of patients with a diagnosis of PD in the population suffer from a different disorder, and approximately $20 \%$ of patients with PD who have already come to medical attention have not been diagnosed as such. The main differential diagnoses are non-parkinsonian tremor, vascular parkinsonism, and atypical parkinsonian disorders. These diagnostic problems should be considered when results of different prevalence studies of IPD are compared.

\subsection{The clinical features and natural history of parkinsonian disorders}

Sufficient data for analysis of clinical features and treatment complications, degree of disability and rate of progression, and existence of subtypes were available only in the group of patients with PD. In this population, falls with postural instability was among the most common complications of advancing disease (64\%). Motor complications of treatment, on the other hand, only occurred in less than $30 \%$. The features that contributed most to disability were disease severity, depression and dementia, whereas motor fluctuations and dyskinesias had less impact on disability in the overall sample. The rate of progression was best predicted by age at onset and development of dementia, but levodopa appeared to provide slowing of symptom progression.

Conclusion and implications: The most disabling problems in the population of patients with PD in the community are disease severity, depression and dementia, whereas motor complications have less impact on the majority of patients. Unfavourable prognostic factors are older age at onset and development of dementia. These factors should be taken into account for the provision of health care resources, and in treatment plans for patients with PD. 


\subsection{Dyskinesias and motor fluctuations in patients with Parkinson's disease}

Fluctuations in motor response and dyskinesias constitute a major problem in long-term treatment of PD. This study investigated the rate of motor fluctuations and dyskinesias, and factors determining their occurrence in the population of patients with PD. Among levodopa-treated patients $28 \%$ suffered from treatment induced dyskinesias and $41 \%$ from response fluctuations. The occurrence of these complications increased with duration of disease and treatment, and with severity of disease. Patients with a shorter time from symptom onset to initiation of levodopa had developed dyskinesias and response fluctuations earlier. The overall rate of motor fluctuations was best predicted by disease duration and dose of levodopa, whereas dyskinesias could be best predicted by duration of treatment. In this population-based sample, age, sex, onset age and symptom at onset were not significant factors in the development of motor fluctuations or dyskinesias. Although a satisfactory response to medication was associated with higher rates of motor complications, poor or moderate response was associated with lower quality of life in patients with a disease duration of less than 5 or more than 10 years.

Conclusion and implications: The rate of motor complications in PD increases with disease duration and severity. Motor fluctuations are more strongly related to disease duration and dose of levodopa, and dyskinesias more to duration of levodopa treatment. However, poorer quality of life associated with inadequate dosage of levodopa may be the price to pay for a low rate of motor complications in patients with PD.

\subsection{Depression in PD}

Depression is a common problem in patients with PD. However, previous studies have disagreed not only on the cause for, but also on the rate of depression in this population. I therefore analysed the rate of depression in this population-based sample of patients with $\mathrm{PD}$ and investigated the factors that contribute to depression in them. Moderate to severe depression (BDI $>17)$ was reported by $19.5 \%$ of patients. Increased depression scores were associated with advancing disease severity $(\mathrm{p}<0.001)$, recent deterioration as reported by the patient $(p<0.001)$, higher akinesia scores $(p<0.005)$, a mini mental score of less than $25(\mathrm{p}<0.01)$, and occurrence of falls $(\mathrm{p}<0.01)$. Objective disability as measured by the Schwab and England scale accounted for $34 \%$ of the 
variance of depression scores. In contrast, subjective impairment of cognitive function and the feeling of stigmatisation accounted for more than $50 \%$ of the variance of depression scores.

Conclusion and implications: Depression in patients with PD is associated with advancing disease severity, recent disease deterioration and occurrence of falls. However, it is strongly influenced by the patients' own perceptions of their handicap rather the result of objective impairment. The treatment of depression should therefore be addressed independently of treatment of motor symptoms of the disease, and consider the patients' own perception of their disease.

\subsection{Subtypes of PD}

$\mathrm{PD}$ has been suggested to be a heterogeneous disorder rather than a single disease. This study assessed the existence of subtypes in the studied population. The clinical features of patients with previously proposed clinical subtypes of PD were compared, and a cluster analysis performed entering all variables that had differed between these groups. Age at onset, predominance type, current age, presence of dementia and rate of progression differentiated patients within this sample of patients with PD, whereas gender, symptom at onset, family history, occurrence of depression or hallucinations were not useful discriminators. Cluster analysis revealed 3 subgroups of patients: 1) Patients with young mean onset and current age, who were more disabled after a longer disease duration than patients in the other groups, but had a slower rate of progression and better cognitive scores. 2) Patients with older age at onset and current age, who had a faster rate of progression, and were more often demented, but developed motor fluctuations less frequently. 3) An intermediate group with age at onset, current age, rate of progression, levodopa dose, disease progression and dementia in between the other two groups.

Conclusions and implications: This study found evidence for the existence of different subtypes of PD in the unselected population of patients with PD. Older age at onset and current age and occurrence of dementia were associated with a faster rate of progression but a lower rate of motor fluctuations, whereas presence of depression, symptoms at onset, gender or family history were not discriminating factors between subgroups. 


\subsection{Young Onset Parkinson's Disease - Clinical features, natural history and mortality}

In a separate sample the clinical features, natural history and mortality of patients with PD starting before age 40 (YOPD) were analysed, including a subgroup of 60 prospectively studied patients. In the group of patients with juvenile parkinsonism (onset before age 21 ), $50 \%$ had a positive family history of parkinsonism in a first degree relative and clinical presentation was heterogeneous. Mortality risk was threefold compared to the normal population (standardised mortality ratio (SMR) 3.1, 95\% CIs 0.64-9.1). In the YOPD group (onset between age 21 and 39), mortality was twofold compared to the normal population (SMR 2.1 with 95\% CIs 1.4-3.2), and threefold in the prospectively studied subgroup (SMR 2.9 with 95\% CIs 1.78-4.40). Poor initial response to 1-dopa was a risk factor for early death $(\mathrm{p}<0.001)$. After a median disease duration of 18 years, clinically significant cognitive impairment had occurred in only $19 \%$ of YOPD patients. It had developed in only $13 \%$ of those still below the age of 60 years, but in $43 \%$ of patients above that age. Age was the most important factor for development of dementia, but, among the factors at onset of parkinsonism, female sex and positive family history of parkinsonism also had, more modest, predictive value. After a disease duration of 10 years or less only $5 \%$ of patients were experiencing falls and 30\% freezing, but all patients had developed levodopa related motor complications and dyskinesias.

Conclusion and implications: The mortality in parkinsonism starting before the age of 40 is increased in comparison to the normal population and similar to the general PD population. Intellectual function and postural reflexes are usually well preserved for many years despite a long history of parkinsonism and the early and frequent occurrence of treatment complications, provided the patients are still biologically and chronologically young.

\subsection{Quality of life in Parkinson's disease}

9.9.1. Comparison of different quality of life measures in patients with Parkinson's disease 
This study tested the feasibility and validity of the EQ-5D to assess QoL in this population-based sample of patients with $\mathrm{PD}$. The response rate was $78 \%$ and the completion rate of the EQ-5D among responders was $96 \%$. The EQ-5D summary index correlated strongly with the PDQ-39 $(r=-0.75, \mathrm{p}<0.001)$ as well as the physical score of the SF $36(r=0.61, p<0.001)$. There was a significant correlation of the EQ-5D summary index with disease severity, as measured by Hoehn and Yahr stage of illness, the Schwab and England disability scale, the motor section of the UPDRS and the depression score. The EQ-5D summary index was also able to distinguish between patients with and without depression, falls, postural instability, cognitive impairment hallucinations, and those with deterioration of health over the previous year.

Conclusion and implications: The EQ-5D is a feasible and valid instrument to measure QoL in PD and reflects severity and complications of disease.

\subsubsection{The impact of Parkinson's disease on quality of life}

QoL in this population-based sample of patients with PD in different disease stages was assessed and compared to that reported in the general population. QoL as measured by the PDQ-39, the EQ-5D and the physical summary of the SF 36 deteriorated significantly with increasing disease severity. The greatest impairment was seen in the areas related to physical and social functioning, whereas reports of pain and poor emotional adjustment had similar prevalence in PD patients and the general population. The impairment of QoL life was seen in all age groups and was similar for men and for women, but the differences between PD patients and the general population were most marked in the younger patient groups.

Conclusions and implications: PD interferes with various aspects of QoL, particularly those related to physical and social functioning. This information should be taken into account in the clinical management and planning and allocation of health care resources to this population.

\subsubsection{Factors that contribute to quality of life in patients with Parkinson's disease} Quality of life is increasingly recognised as a critical measure in health care which incorporates patients' own perspective of their health. This study identified the factors that determine quality of life in patients with idiopathic PD. The factor most closely 
associated with QoL was the presence of depression, but disability, as measured by the Schwab and England scale, postural instability, and cognitive impairment additionally contributed to poor QoL. Although the UPDRS part III correlated significantly with QoL scores, it did not contribute substantially to predicting their variance once depression, disability, and postural instability had been taken into account. In addition, patients with akinetic-rigid PD had worse QoL scores than those with tremor-dominant $\mathrm{PD}$, mainly due to impairment of axial features.

Conclusion and implications: Depression, disability, postural instability and cognitive impairment have the greatest influence on QoL in $\mathrm{PD}$. The improvement of these features should therefore become a greater target in the treatment of PD. 
Ben-Shlomo Y, Marmot MG: Survival and cause of death in a cohort of patients with parkinsonism: possible clues to aetiology? J Neurol Neurosurg Psychiatry 1995;58:293299

Bharucha NE, Bharucha EP, Bharucha AE, Bhise AV, Schoenberg BS. Prevalence of Parkinson's disease in the Parsi community of Bombay, India. Arch Neurol $1988 ; 45: 1321-3$

Biggins CA, Boyd JL, Harrop FM, et al. A controlled, longitudinal study of dementia in Parkinson's disease. J Neurol Neurosurg Psychiatry 1992;55:566-71

Blin J, Bonnet AM, Agid Y. Does levodopa aggravate Parkinson's disease? Neurology 1988;38:1410-6

Blin J, Dubois B, Bonnet AM, Vidailhet M, Brandabur M, Agid Y. Does ageing aggravate parkinsonian disability? J Neurol Neurosurg Psychiatry 1991;54:780-2 Block G, Liss C, Reines S, Irr J, Nibbelink D. Comparison of immediate-release and controlled release carbidopa/levodopa in Parkinson's disease. A multicenter 5-year study. The CR First Study Group. Eur Neurol 1997;37:23-7

Boller F, Mizutani T, Roessmann U, Gambetti P. Parkinson's disease, dementia and Alzheimer's disease:clinicopathological correlations. Ann Neurol 1980;7:329-335

Bonifati V, Fabrizio E, Vanacore N, De Mari M, Meco G. Familial Parkinson's disease: a clinical genetic analysis. Can J Neurol Sci 1995;22:272-9

Bower JH, Maraganore DM, McDonnell SK, Rocca WA. Incidence of progressive supranuclear palsy and multiple system atrophy in Olmsted County, Minnesota, 1976 to 1990 . Neurology $1997 ; 49: 1284-8$

Boyce S, Clarke CE, Luquin R, Peggs D, Robertson RG, Mitchell IJ, Sambrook MA, Crossman AR. Induction of chorea and dystonia in parkinsonian primates. Mov Disord $1990 \mathrm{~b} ; 5: 3-7$

Boyce S, Rupniak NM, Steventon MJ, Iversen SD. Nigrostriatal damage is required for induction of dyskinesias by L-DOPA in squirrel monkeys. Clin Neuropharmacol 1990a; 13:448-58

Brandt J, Spencer M, Folstein M: The telephone interview for cognitive status.

Neuropsychiatr Neuropsychology Behav Neurol 1988; 1: 111-117.

Brannan T, Yahr MD . Comparative study of selegiline plus L-dopa-carbidopa versus 
L-dopa-carbidopa alone in the treatment of Parkinson's disease. Ann Neurol 1995;37:95-8

Brazier JE, Harper R, Jones NM, O'Cathain A, Thomas KJ, Usherwood T, et al. Validating the SF-36 health survey questionnaire: new outcome measure for primary care. BMJ 1992;305:160-4

Brazier JE, Walters SJ, Nicholl JP, Kohler B. Using the SF-36 and Euroqol on an elderly population. Qual Life Res 1996;5:195-204

Brewis M, Poskanzer DC, Rolland C, Miller H. Neurological disease in an English city. Acta Neurol Scand 1966;42:Suppl 24:1-89

Broman T. Parkinson syndrome, prevalence and incidence in Gotenborg. Acta Neurol Scand 1963;399(suppl 4):95-101

Brooks R, with the EuroQol Group. EuroQol: the current state of play. Health Policy 1996;37:53-72

Brown R, Jahanshahi M. Depression in Parkinson's disease: a psychosocial viewpoint. Adv Neurol 1995;65:61-84

Brown RG, MacCarthy B, Jahanshahi M, Marsden CD. Accuracy of self-reported disability in patients with parkinsonism. Arch Neurol 1989;46:955-9

Brown RG, Marsden CD. How common is dementia in Parkinson's disease? Lancet $1984 ; 2: 1262-5$

Calne DB. Is "Parkinson's disease" one disease? J Neurol Neurosurg Psychiatry 1989;Suppl:18-21

Cantello R, Gilli M, Riccio A, Bergamasco B. Mood changes associated with "end-ofdose deterioration" in Parkinson's disease: a controlled study. J Neurol Neurosurg Psychiatry 1986;49:1182-90

Caraceni T, Giovannini P, Girotti F, Grassi MP, Piccolo I, Soliveri P. Juvenile parkinsonism: a different clinical expression of Parkinson's disease. J Neurol 1985;232 suppl:66

Cedarbaum JM, Gandy SE, McDowell FH . "Early" initiation of levodopa treatment does not promote the development of motor response fluctuations, dyskinesias, or dementia in Parkinson's disease. Neurology 1991;41:622-9 
Celesia GG, Wanamaker WM. Psychiatric disturbances in Parkinson's disease. Dis Nerv Syst 1972;33:577-83

Charlton CG. Depletion of nigrostriatal and forebrain tyrosine hydroxylase by Sadenosylmethionine: a model that may explain the occurrence of depression in Parkinson's disease. Life Sci 1997;61:495-502

Chetter IC, Spark JI, Dolan P, Scott DJ, Kester RC. Quality of life analysis in patients with lower limb ischaemia: suggestions for European standardisation. Eur J Vasc Endovasc Surg 1997;13:597-604

Chio A, Magnani C, Schiffer D. Prevalence of Parkinson's disease in Northwestern Italy: comparison of tracer methodology and clinical ascertainment of cases. Mov Disord 1998;13:400-5

Chouinard S, Louis ED, Fahn S. Agreement among movement disorder specialists on the clinical diagnosis of essential tremor. Mov Disord 1997;12:973-6

Chrischilles EA, Rubenstein LM, Voelker MD, Wallace RB, Rodnitzky RL . The health burdens of Parkinson's disease. Mov Disord 1998;13:406-13

Clayton D, Hills M. Statistical methods in epidemiology. Oxford University Press 1993, Oxford

Cockerell OC, Goodridge DM, Brodie D, Sander JW, Shorvon SD. Neurological disease in a defined population: the results of a pilot study in two general practices.

Neuroepidemiology 1996;15:73-82

Cockerell OC; Sander JW; Shorvon SD. Neuroepidemiology in the United Kingdom J Neurol Neurosurg Psychiatry 1993;56:735-8

Crystal HA, Dickson DW, Lizardi JE, Davies P, Wolfson LI. Antemortem diagnosis of diffuse Lewy body disease. Neurology 1990;40:1523-1528

Cummings JL. Depression and Parkinson's disease: a review. Am J Psychiatry 1992;149:443-54

D'Alessandro R, Gamberini G, Granieri E, Benassi G, Naccarato S, Manzaroli D. Prevalence of Parkinson's disease in the Republic of San Marino. Neurology 1987;37:1679-82

Davison D: Pallido-pyramidal disease. J Neuropathol Exp Neurol 1947;13:50-59 
de Boer AG, Wijker W, Speelman JD, de Haes JC . Quality of life in patients with Parkinson's disease: development of a questionnaire. J Neurol Neurosurg Psychiatry 1996;61:70-4

de Jong GJ, Meerwaldt JD, Schmitz PI. Factors that influence the occurrence of response variations in Parkinson's disease. Ann Neurol 1987;22:4-7 de Rijk MC, Breteler MM, Graveland GA, Ott A, Grobbee DE, van der Meche FG, Hofman A. Prevalence of Parkinson's disease in the elderly: the Rotterdam Study. Neurology 1995;45:2143-6 de Rijk MC, Tzourio C, Breteler MM, Dartigues JF, Amaducci L, Lopez-Pousa S, Manubens-Bertran JM, Alperovitch A, Rocca WA. Prevalence of parkinsonism and Parkinson's disease in Europe: the EUROPARKINSON Collaborative Study. European Community Concerted Action on the Epidemiology of Parkinson's disease. J Neurol Neurosurg Psychiatry 1997;62:10-5

Denny AP, Behari M. Motor fluctuations in Parkinson's disease. J Neurol Sci $1999 ; 165: 18-23$

Devinsky O. Outcome research in neurology: incorporating health-related quality of life. Ann Neurol 1995;37:141-2

Diamond SG, Markham CH, Hoehn MM, McDowell FH, Muenter MD. Effect of age at onset on progression and mortality in Parkinson's disease. Neurology 1989;39:1187-90 Dorman PJ, Waddell F, Slattery J, Dennis M, Sandercock P . Is the EuroQol a valid measure of health-related quality of life after stroke? Stroke 1997;28:1876-82 Dupont E, Andersen A, Boas J, Boisen E, Borgmann R, Helgetveit AC, Kjaer MO, et al. Sustained-release Madopar HBS compared with standard Madopar in the long-term treatment of de novo parkinsonian patients. Acta Neurol Scand 1996;93:14-20 Duvoisin RC. Is there a Parkinson's Disease? In: Quinn NP, Jenner PG, eds. Disorders of Movement. Clinical, pharmacological and physiological aspects. London: Academic Press, 1989.

Ebmeier KP, Calder SA, Crawford JR, Stewart L, Cochrane RH, Besson JA. Dementia in idiopathic Parkinson's disease: prevalence and relationship with symptoms and signs of parkinsonism. Psychol Med 1991;21:69-76 
Ehmann TS, Beninger RJ, Gawel MJ, Riopelle RJ. Depressive symptoms in

Parkinson's disease: a comparison with disabled control subjects. J Geriatr Psychiatry Neurol 1990;3:3-9

EuroQoL Group. EuroQoL: a new facility for the measurement of health-related quality of life. Health Policy 1990;16:199-208

Eurostat Data Shop, Office for National Statistics, 1Drummond Gate, London SW1V 2QQ

Fahn S, Bressman SB. Should levodopa therapy for Parkinsonism be started early or late? Evidence against early treatment. Can J Neurol Sci 1984;11(1 Suppl):200-5 Fahn S, Elton RL, members of the UPDRS Development Committee. Unified Parkinson's Disease Rating Scale. In: Fahn S, Marsden CD, Calne DB, Goldstein M, eds. Recent developments in Parkinson's Disease, Vol 2. Florham Park NJ: MacMillan Healthcare Information, 1987:153-163

Fahn S. Adverse effects of levodopa. In: Olanow CW, Lieberman AN, eds. The Scientific Basis for the Treatment of Parkinson's disease. Carnforth: Parthenon Publishing Group, 1992:89-112

Fetoni V, Soliveri P, Monza D, Testa D, Girotti F. Affective symptoms in multiple system atrophy and Parkinson's disease: response to levodopa therapy. J Neurol Neurosurg Psychiatry 1999;66:541-4

Fitzpatrick R, Jenkinson C, Peto V, Hyman N, Greenhall R. Desirable properties for instruments assessing quality of life: evidence from the PDQ-39. J Neurol Neurosurg Psychiatry $1997 b ; 62: 104$

Fitzpatrick R, Newman S, Lamb R, Shipley M. Social relationships and psychological well-being in rheumatoid arthritis. Soc Sci Med 1988;27:399-403

Fitzpatrick R, Peto V, Jenkinson C, Greenhall R, Hyman N. Health-related quality of life in Parkinson's disease: a study of outpatient clinic attenders. Mov Disord $1997 \mathrm{a} ; 12: 916-22$

Folstein MF, Folstein SE, McHugh PR. "Mini-mental state". A practical method for grading the cognitive state of patients for the clinician. J Psychiatr Res 1975;12:189-98 Forno LS. Pathology of parkinsonism: A preliminary report. of 24 cases. J Neurosurg $1966 ; 24: 266-271$ 
Friedman A: Old-onset Parkinson's disease compared with young-onset disease: clinical differences and similarities. Acta Neurol Scand 1994;89:258-261

Gaspar P, Gray F. Dementia in idiopathic Parkinson's disease. A neuropathological study of 32 cases. Acta Neuropathol 1984;64:43-52

Gershanik OS, Leist A. Juvenile onset Parkinson's disease. Adv Neurol 1986;45:213216

Gibb WR, Luthert PJ, Marsden CD. Clinical and pathological features of corticobasal degeneration. Adv Neurol 1990;53:51-4

Gibb WR, Luthert PJ, Marsden CD. Corticobasal degeneration. Brain 1989a;112:117192

Gibb WR; Lees AJ . The significance of the Lewy body in the diagnosis of idiopathic Parkinson's disease. Neuropathol Appl Neurobiol. 1989b;15:27-44

Gibb WRG, Lees AJ: A comparison of clinical and pathological features of young- and old-onset Parkinson's disease. Neurology 1988; 38:1402-1406

Giovannini P, Piccolo I, Genitrini S, et al. Early-onset Parkinson's disease. Mov Disord $1991 ; 6: 36-42$

Goetz CG, Stebbins GT. Mortality and hallucinations in nursing home patients with advanced Parkinson's disease. Neurology 1995;45:669-71

Goetz CG, Stebbins GT. Risk factors for nursing home placement in advanced Parkinson's disease. Neurology 1993;43:2227-9

Goetz CG, Vogel C, Tanner CM, Stebbins GT. Early dopaminergic drug-induced hallucinations in parkinsonian patients. Neurology 1998;51:811-4

Golbe LI, Davis PH, Schoenberg BS, Duvoisin RC. Prevalence and natural history of progressive supranuclear palsy. Neurology 1988;38:1031-4

Gotham AM, Brown RG, Marsden CD. Depression in Parkinson's disease: a quantitative and qualitative analysis. J Neurol Neurosurg Psychiatry 1986;49:381-9 Gourie-Devi M, Ramu MG, Venkataram BS. Treatment of Parkinson's disease in 'Ayurveda' (ancient Indian system of medicine): discussion paper. J R Soc Med 1991;84:491-2

Graham JG, Oppenheimer DR. Orthostatic hypotension and nicotine sensitivity in a case of multiple system atrophy. J Neurol Neurosurg Psychiatry 1969;32:28-34 
Graham JM, Sagar HJ. A data-driven approach to the study of heterogeneity in idiopathic Parkinson's disease: identification of three distinct subtypes. Mov Disord 1999;14:10-20

Gudmundsson KR. A clinical survey of parkinsonism in Iceland. Acta Neurol Scand 1967;43:Suppl 33:1-61

Hakim AM, Mathieson G. Dementia in Parkinson's disease. A neuropathological study. Neurology 1979;29:1209-1214

Harada H, Nishikawa S, Takahashi K. Epidemiology of Parkinson's disease in a Japanese city. Arch Neurol 1983 Mar;40:151-4

Hauw JJ, Daniel SE, Dickson D, et al. Preliminary NINDS neuropathologic criteria for Hely MA, Morris JG, Reid WG, O'Sullivan DJ, Williamson PM, Rail D, Broe GA, et al. The Sydney Multicentre Study of Parkinson's disease: a randomised, prospective five year study comparing low dose bromocriptine with low dose levodopa carbidopa. J Neurol Neurosurg Psychiatry 1994;57:903-10

Hershey LA, Feldman BJ, Kim KY, Commichau C, Lichter DG. Tremor at onset. Predictor of cognitive and motor outcome in Parkinson's disease? Arch Neurol 1991;48:1049-51

Hietanen M, Teravainen $\mathrm{H}$. The effect of ageing and onset on neuropsychological performance in Parkinson's disease. J Neurol Neurosurg Psychiatry 1988;51:244-249 Hoehn MM, Yahr MD. Parkinsonism: onset, progression and mortality. Neurology $1967 ; 17: 427-42$

Horowitz G, Greenberg J. Pallido-pyramidal syndrome treated with levodopa. J Neurol Neurosurg Psychiatry 1975;38:238-240

Horowitz JR. Progressive atrophy of the globus pallidus. Brain 1917;40:58-148 Huber SJ, Paulson GW, Shuttleworth EC. Relationship of motor symptoms, intellectual impairment, and depression in Parkinson's disease. J Neurol Neurosurg Psychiatry $1988 ; 51: 855-8$

Hughes AJ, Ben-Shlomo Y, Daniel SE, Lees AJ. What features improve the accuracy of clinical diagnosis in Parkinson's disease: a clinicopathologic study. Neurology $1992 ; 42: 1142-6$ 
Hurst NP, Jobanputra P, Hunter M, Lambert M, Lochhead A, Brown H. Validity of Euroqol--a generic health status instrument--in patients with rheumatoid arthritis. Economic and Health Outcomes Research Group. Br J Rheumatol 1994;33:655-62 Ishikawa A, Tsuji S. Clinical analysis of 17 patients in 12 Japanese families with autosomal-recessive type juvenile parkinsonism. Neurology 1996;47:160-166 Izumi K, Kuroiwa Y. Parkinsonism: clinical observations on 183 cases. Naika. 1971;27:311-306

Jahanshahi M, Marsden CD. Depression in torticollis: a controlled study. Psychol Med $1988 ; 18: 925-33$

Jankovic J, McDermott M, Carter J, et al. Variable expression of Parkinson's disease: a base-line analysis of the DATATOP cohort. The Parkinson Study Group. Neurology 1990;40:1529-34

Jenkinson C, Coulter A, Wright L. Short form 36 (SF 36) health survey questionnaire: normative data for adults of working age. BMJ 1993;306:1437-40

Jenkinson C, Fitzpatrick R, Peto V, Greenhall R, Hyman N. The Parkinson's Disease Questionnaire (PDQ-39): development and validation of a Parkinson's disease summary index score. Age Ageing 1997;26:353-7

Jenkinson C, Peto V, Fitzpatrick R, Greenhall R, Hyman N. Self-reported functioning and well-being in patients with Parkinson's disease: comparison of the short-form health survey (SF-36) and the Parkinson's Disease Questionnaire (PDQ-39). Age Ageing 1995;24:505-9

Kalra S, Bergeron C, Lang AE. Lewy body disease and dementia. A review. Arch Intern Med 1996;156:487-93

Karlsen KH, Larsen JP, Tandberg E, Maeland JG. Influence of clinical and demographic variables on quality of life in patients with Parkinson's disease. J Neurol Neurosurg Psychiatry 1999;66:431-5

Kind P, Dolan P, Gudex C, Williams A . Variations in population health status: results from a United Kingdom national questionnaire survey. BMJ 1998;316:736-41

King RB. Quality of life after stroke. Stroke 1996;27:1467-72 
Kostic V, Przedborski S, Flaster E, Sternic N: Early development of 1-dopa induced dyskinesias and response fluctuations in young onset Parkinson's disease. Neurology 1991;41: 202-205

Lang AE, Lozano AM . Parkinson's disease. Second of two parts. N Engl J Med 1998;339:1130-43

Lantos PL. The definition of multiple system atrophy: a review of recent developments. J Neuropathol Exp Neurol 1998;57:1099-111

Lees AJ, Stern GM . Sustained bromocriptine therapy in previously untreated patients with Parkinson's disease. J Neurol Neurosurg Psychiatry 1981;44:1020-3 Lesser RP, Fahn S, Snider SR, Cote LJ, Isgreen WP, Barrett RE . Analysis of the clinical problems in parkinsonism and the complications of long-term levodopa therapy. Neurology 1979;29:1253-60

Levin BE, Llabre MM, Weiner WJ. Parkinson's disease and depression: psychometric properties of the Beck Depression Inventory. J Neurol Neurosurg Psychiatry $1988 ; 51: 1401-4$

Li SC, Schoenberg BS, Wang CC, Cheng XM, Rui DY, Bolis CL, Schoenberg DG. A prevalence survey of Parkinson's disease and other movement disorders in the People's Republic of China. Arch Neurol 1985;42:655-7

Lieberman A, Dziatolowski M, Kupersmith M, et al: Dementia in Parkinson's disease. Ann Neurol 1979; 6: 355-359.

Lieberman A. Managing the neuropsychiatric symptoms of Parkinson's disease. Neurology 1998;50(Suppl 6):S33-8

Litvan I, Agid Y, Calne D, et al. Clinical research criteria for the diagnosis of progressive supranuclear palsy (Steele-Richardson-Olszewski syndrome): report of the NINDS-SPSP international workshop. Neurology 1996a;47:1-9

Litvan I, Agid Y, Goetz C, Jankovic J, et al. Accuracy of the clinical diagnosis of corticobasal degeneration: a clinicopathologic study. Neurology 1997b;48:119-25 Litvan I, MacIntyre A, Goetz CG, et al. Accuracy of the clinical diagnoses of Lewy body disease, Parkinson disease, and dementia with Lewy bodies: a clinicopathologic study. Arch Neurol 1998;55:969-78 
Litvan I, Mangone CA, McKee A, et al. Natural history of progressive supranuclear palsy (Steele-Richardson-Olszewski syndrome) and clinical predictors of survival: a clinicopathological study. J Neurol Neurosurg Psychiatry 1996b;60:615-20

Litvan I. Progressive supranuclear palsy and corticobasal degeneration. In: Quinn NP, ed. Clinical Neurology. London: Baillièrre Tindall, 1997a.

Livingston G, Watkin V, Milne B, Manela MV, Katona C. The natural history of depression and the anxiety disorders in older people: the Islington community study. $\mathrm{J}$ Affect Disord 1997;46:255-62

Ludin SM, Ludin HP: Is Parkinson's disease of early onset a separate disease entity? J Neurol 1989;236:203-207

Lyons KE, Hubble JP, Troster AI, Pahwa R, Koller WC . Gender differences in Parkinson's disease. Clin Neuropharmacol 1998;21:118-21

MacCarthy B, Brown R. Psychosocial factors in Parkinson's disease. Br J Clin Psychol $1989 ; 28: 41-52$

Manyam BV. Paralysis agitans and levodopa in "Ayurveda": ancient Indian medical Maraganore DM, Anderson DW, Bower JH, McDonnell SK, Rocca WA. Potential bias in autopsy series of Parkinson's disease and related disorders. Neurology 1998;50:A98 Markham CH, Diamond SG . Long-term follow-up of early dopa treatment in Parkinson's disease. Ann Neurol 1986;19:365-72

Marsden CD, Parkes JD . Success and problems of long-term levodopa therapy in Parkinson's disease. Lancet 1977;12;1:345-9

Martinez-Martin P, Frades Payo B. Quality of life in Parkinson's disease: validation study of the PDQ-39 Spanish version. The Grupo Centro for Study of Movement Disorders. J Neurol 1998;(Suppl 1):S34-8

Marttila RJ, Rinne UK. Epidemiology of Parkinson's disease in Finland. Acta Neurol Scand 1976;53:81-102

Marttila RJ, Rinne. Disability and progression in Parkinson's disease. Acta Neurol Scand 1977;56:159-69

Mastaglia FL, Grainger K, Kee F, Sadka M, Lefroy R. Progressive supranuclear palsy (the Steele-Richardson-Olszewski syndrome) clinical and electrophysiological observations in eleven cases. Proc Aust Assoc Neurol 1973;10:35-44 
Mayeux R, Denaro J, Hemenegildo N, et al. A population-based investigation of Parkinson's disease with and without dementia. Relationship to age and gender. Arch Neurol 1992;49:492-7

Mayeux R, Stern Y, Cote L, Williams JB. Altered serotonin metabolism in depressed patients with Parkinson's disease. Neurology 1984;34:642-6

Mayeux R, Stern Y, Rosen J, Leventhal J. Depression, intellectual impairment, and Parkinson disease. Neurology 1981;32:645-50

Mayeux R, Stern Y, Rosenstein R, et al . An estimate of the prevalence of dementia in idiopathic Parkinson's disease. Arch Neurol 1988;45:260-2

Mayeux R, Stern Y, Williams JB, Cote L, Frantz A, Dyrenfurth I. Clinical and biochemical features of depression in Parkinson's disease. Am J Psychiatry 1986;143:756-9

McGuire D, Michalek D, Koller WC. Motor complications of chronic levodopa therapy in Parkinson's disease. Clin Neuropharmacol 1997 Dec;20:523-30

McHorney CA, Ware JE Jr, Lu JF, Sherbourne CD . The MOS 36-item Short-Form Health Survey (SF-36): III. Tests of data quality, scaling assumptions, and reliability across diverse patient groups. Med Care 1994;32:40-66

McHorney CA, Ware JE Jr, Raczek AE. The MOS 36-Item Short-Form Health Survey (SF-36): II. Psychometric and clinical tests of validity in measuring physical and mental health constructs. Med Care 1993;31:247-63

McIvor GP, Riklan M, Reznikoff M. Depression in multiple sclerosis as a function of length and severity of illness, age, remissions, and perceived social support. J Clin Psychol 1984;40:1028-33

Meara J, Bhowmick BK, Hobson P. Accuracy of diagnosis in patients with presumed Parkinson's disease. Age Ageing 1999;28:99-102

measures. J Neurol 1998;245(Suppl 1):S10-4

Melamed E. Initiation of levodopa therapy in parkinsonian patients should be delayed until the advanced stages of the disease. Arch Neurol 1986;43:402-5

Mindham RH, Bagshaw A, James SA, Swannell AJ. Factors associated with the appearance of psychiatric symptoms in rheumatoid arthritis. J Psychosom Res $1981 ; 25: 429-35$ 
Miyawaki E, Lyons K, Pahwa R, Troster AI, Hubble J, Smith D, Busenbark K, Mizutima Y, Anno M, Yokochi M, Narabayashi H. Clinicopathological analysis of ten autopsy cases of juvenile parkinsonism. Abstract book, 10th Int. Symp on Parkinson's disease. Tokyo Oct 27-31 1991a, p224

Mizutima Y, Yokochi M, Ozanagi S. Juvenile parkinsonism: a case with first clinical manifestation at the age of six years and with neuropathological findings suggesting a new pathogenesis. Clin Neuropathol 1991b;10:91-97

Montastruc JL, Rascol O, Senard JM, Rascol A. A randomised controlled study comparing bromocriptine to which levodopa was later added, with levodopa alone in previously untreated patients with Parkinson's disease: a five year follow up. J Neurol Neurosurg Psychiatry 1994;57:1034-8

Moos RH, Solomon GF. Personality correlates of the rapidity of progression of rheumatoid arthritis. Ann Rheum Dis 1964;23:145-151

Morgante L, Rocca WA, Di Rosa AE et al. Prevalence of Parkinson's disease and other types of parkinsonism: a door-to-door survey in three Sicilian municipalities. The Sicilian Neuro-Epidemiologic Study (SNES) Group. Neurology 1992;42:1901-7 Mutch WJ, Dingwall-Fordyce I, Downie AW, Paterson JG, Roy SK. Parkinson's disease in a Scottish city. Br Med J 1986a;292:534-6

Mutch WJ; Strudwick A; Roy-SK; Downie AW. Parkinson's disease: disability, review, and management. Br Med J 1986b; 293: 675-7

Muthane EB, Swamy HS, Satishchandra P, Subash MN, Rao S, Subbakrishna D. Early onset Parkinson's disease: Are juvenile and young onset different? Mov Disord 1994;5:539-544

Nobrega FT, Glattre E, Kurland LT, Okazaki H. Comments on the epidemiology of parkinsonism including prevalence and incidence statsitics for Rochester, Minnesota 1935-1966. In: Barbeau A, Brunette JR, eds. Progress in Neurogenetics. Proceedings of the second International Congress of Neurogenetics and Neuro-Ophtalmology.

Amsterdam: Excerpta Medica, 1969.

Office of Population Censuses and Surveys. Classification of occupations and coding index, 1980. HMSO, 1980 
Okada K, Kobayashi S, Tsunematsu T. Prevalence of Parkinson's disease in Izumo City, Japan. Gerontology 1990;36:340-4

Pantelatos A, Fornadi F. Clinical features and medical treatment of Parkinson's disease in patient groups selected in accordance with age at onset. Adv Neurol 1993;60:690697

Parkinson Study Group. Impact of deprenyl and tocopherol treatment on Parkinson's disease in DATATOP patients requiring levodopa. Ann Neurol 1996;39:37-45

Payami H, Larsen K, Bernard S, Nutt J. Increased risk of Parkinson's disease in parents and siblings of patients. Ann Neurol 1994;36:659-61

Pederzoli M, Girotti F, Scigliano G, Aiello G, Carella F, Caraceni T . L-dopa long-term treatment in Parkinson's disease: age-related side effects. Neurology 1983;33:1518-22 Peto V, Jenkinson C, Fitzpatrick R, Greenhall R. The development and validation of a short measure of functioning and well being for individuals with Parkinson's disease. Qual Life Res 1995;4:241-8

Poewe WH, Lees AJ, Stern GM. Low-dose L-dopa therapy in Parkinson's disease: a 6 year follow-up study. Neurology 1986;36:1528-30

Poewe WH, Wenning GK. The natural history of Parkinson's disease. Ann Neurol 1998;44(Suppl 1):S1-9

Quinn N, Critchley P, Marsden CD: Young onset Parkinson's disease. Mov Disord 1987;2: 73-91

Quinn N. Multiple system atrophy--the nature of the beast. J Neurol Neurosurg Psychiatry 1989;Suppl:78-89

Quinn NP. Multiple system atrophy. In: Marsden CD, Fahn F, eds. Movement Disorders 3. Oxford: Butterworth-Heinemann, 1994:261-80

Quinn NP, Rossor MN, Marsden CD. Dementia and Parkinson's disease - pathological and neurochemical considerations. Br Med Bull 1986;42:86-90

Quinn NP. Classification of fluctuations in patients with Parkinson's disease.

Neurology 1998;51(Suppl 2):S25-9

Radhakrishnan K, Thacker AK, Maloo JC, Gerryo SE, Mousa ME. Descriptive epidemiology of some rare neurological diseases in Benghazi, Libya.

Neuroepidemiology 1988;7:159-64 
Rajput AH, Birdi S. Epidemiology of Parkinson's disease. Parkinsonism and related disorders 1997;3:175-186

Rajput AH, Offord KP, Beard CM, Kurland LT. Epidemiology of parkinsonism: incidence, classification, and mortality. Ann Neurol 1984;16:278-82

Rajput AH, Rozdilsky B, Rajput A. Accuracy of clinical diagnosis in parkinsonism--a prospective study. Can J Neurol Sci 1991;18:275-8

Ransmayer G, Poewe W, Plorer S, Gerstenbrandt F, Leidlmair K, Mayr U. Prognostic implications of the motor symptoms of Parkinson's disease with respect to clinical, computertomographic and psychometric parameters. J Neural Transm 1986;67:1-14 Rebeiz JJ, Kolodny EH, Richardson EP Jr. Corticodentatonigral degeneration with neuronal achromasia. Arch Neurol 1968;18:20-33

Richards M, Marder K, Cote L, Mayeux R. Reliability of symptom onset assessment in Parkinson's disease. Mov Disord 1994;9:340-2

Ring HA, Bench CJ, Trimble MR, Brooks DJ, Frackowiak RS, Dolan RJ. Depression in Parkinson's disease. A positron emission study. Br J Psychiatry 1994;165:333-9 Rinne UK . Early combination of bromocriptine and levodopa in the treatment of Parkinson's disease: a 5-year follow-up. Neurology 1987;37:826-8

Robins AH. Depression in patients with Parkinsonism. Br J Psychiatry 1976 Feb;128:141-5

Röhl A, Friedrich HJ, Ulm G, Vieregge P. The relevance of clinical subtypes for disease course, family history and epidemiological variables in Parkinson's diease. Eur J Neurol 1994;1:65-72

Roos RA, Jongen JC, van-der-Velde-EA. Clinical course of patients with idiopathic Parkinson's disease. Mov Disord 1996;11:236-42

Roos RA, Vredevoogd CB, van der Velde EA. Response fluctuations in Parkinson's disease. Neurology 1990;40:1344-6

Rosati G, Granieri E, Pinna L, et al. The risk of Parkinson disease in Mediterranean people. Neurology 1980;30:250-5

Rubenstein LM, Voelker MD, Chrischilles EA, Glenn DC, Wallace RB, Rodnitzky RL. The usefulness of the Functional Status Questionnaire and Medical Outcomes Study Short Form in Parkinson's disease research. Qual Life Res 1998;7:279-90 
Rybicki BA, Johnson CC, Gorell JM. Demographic differences in referral rates to neurologists of patients with suspected Parkinson's disease: implications for casecontrol study design. Neuroepidemiology 1995;14:72-81.

Sanchez-Ramos JR, Ortoll R, Paulson GW. Visual hallucinations associated with Parkinson disease. Arch Neurol 1996;53:1265-8

Santamaria J, Tolosa E, Valles A. Parkinson's disease with depression: a possible subgroup of idiopathic parkinsonism. Neurology 1986;36:1130-3

Schneider JS. Levodopa-induced dyskinesias in parkinsonian monkeys: relationship to extent of nigrostriatal damage. Pharmacol Biochem Behav 1989;34:193-6

Schoenberg BS, Anderson DW, Haerer AF. Prevalence of Parkinson's disease in the biracial population of Copiah County, Mississippi. Neurology 1985;35:841-5

Schoenberg BS, Osuntokun BO, Adeuja AO, et al. Comparison of the prevalence of Parkinson's disease in black populations in the rural United States and in rural Nigeria: door-to-door community studies. Neurology 1988;38:645-6

Schoenberg BS. Descriptive neuroepidemiology: applications to occupational neurology. Acta Neurol Scand Suppl 1982;92:1-9

Schoenberg BS. Neuroepidemiology. Incidents, incidence, and coincidence. Arch Neurol 1977;34:261-5

Schoenberg BS. Neurological disease in the elderly: Epidemiologic considerations. Seminars in Neurology. 1981;1:5-12

Schwab RS, England AC. Projection technique for evaluating surgery in Parkinson's disease. In: Gillingham FJ, Donaldson IML, eds. Third Symposium on Parkinson's disease. Edinburgh: Livingstone, 1969: 152-157

Selai, C.E., Elstner, K. \& Trimble, M.R. Is the EuroQol EQ-5D sensitive to clinically defined treatment outcome in epilepsy? Proceedings of the EuroQol Plenary Meeting, Hannover, 1-3 October, 1998. Hannover University (in press)

Senard JM, Rai S, Lapeyre-Mestre M, et al. Prevalence of orthostatic hypotension in Parkinson's disease. J Neurol Neurosurg Psychiatry 1997;63:584-9

Smith P. Comparison between registries: age-standardized rates. In: Muir C, Waterhouse J, Mack J, Powell J, Whelan S, eds. Cancer incidence in five continents. Volume V. Lyon: IARC Scientific Publications No 88, 1987:790-5 
Starkstein SE, Berthier ML, Bolduc PL, Preziosi TJ, Robinson RG. Depression in patients with early versus late onset of Parkinson's disease. Neurology 1989;39:1441-5 Starkstein SE, Mayberg HS, Leiguarda R, Preziosi TJ, Robinson RG. A prospective longitudinal study of depression, cognitive decline, and physical impairments in patients with Parkinson's disease. J Neurol Neurosurg Psychiatry 1992;55:377-82 Starkstein SE, Petracca G, Chemerinski E, et al. Depression in classic versus akineticrigid Parkinson's disease. Mov Disord 1998;13:29-33

Starkstein SE, Preziosi TJ, Bolduc PL, Robinson RG. Depression in Parkinson's disease. J Nerv Ment Dis 1990;178:27-31

Steele JC, Richardson JC, Olszewski J. Progressive supranuclear palsy. A heterogeneous degeneration involving the brain stem, basal ganglia and cerebellum with vertical gaze and pseudobulbar paslsy, nuchal dystonia and dementia. Arch Neurol 1964;10:333-359

Steele-Richardson-Olszewski syndrome (progressive supranuclear palsy). Neurology 1994;44:2015-9

Sutcliffe RL, Meara JR. Parkinson's disease epidemiology in the Northampton District, England, 1992. Acta Neurol Scand 1995;92:443-50

Sutcliffe RL, Prior R, Mawby B, McQuillan WJ. Parkinson's disease in the district of the Northampton Health Authority, United Kingdom. A study of prevalence and disability. Acta Neurol Scand 1985;72:363-79

Sweet RD, McDowell FH . Five years' treatment of Parkinson's disease with levodopa. Therapeutic results and survival of 100 patients. Ann Intern Med 1975;83:456-63 Takahashi H, Ohama E, Suzuki S, et al. Familial juvenile parkinsonism: clinical and pathological study in a family. Neurology 1994;44:437-441

Tandberg E, Larsen JP, Aarsland D, Cummings JL. The occurrence of depression in Parkinson's disease. A community-based study. Arch Neurol 1996;53:175-9

Tandberg E, Larsen JP, Aarsland D, Laake K, Cummings JL. Risk factors for depression in Parkinson disease. Arch Neurol 1997;54:625-30

Tandberg E, Larsen JP, Nessler EG, Riise T, Aarli JA. The epidemiology of Parkinson's disease in the county of Rogaland, Norway. Mov Disord 1995;10:541-9. Tanner CM, Kinori I, Goetz CG, Carvey PM, Klawans HL. Age at onset and clinical 
outcome in idiopathic Parkinson's disease. Neurology 1985a;35(suppl 1):276 Tanner CM, Kinori I, Goetz CG, et al. Clinical course in Parkinson's disease: relationship to age at onset. J Neurol 1985b;232(suppl):25

Tanner CM, Ottman R, Ellenberg JH, et al. Parkinson's disease (PD) concordance in elderly male monozygotic (MZ) and dizygotic (DZ) twins. Neurology 1997;48, suppl:A333

Tison F, Dartigues JF, Dubes L, Zuber M, Alperovitch A, Henry P. Prevalence of Parkinson's disease in the elderly: a population study in Gironde, France. Acta Neurol Scand 1994;90:111-5

treatise. Mov Disord 1990;5:47-8

Trenkwalder C, Schwarz J, Gebhard J, et al. Starnberg trial on epidemiology of Parkinsonism and hypertension in the elderly. Prevalence of Parkinson's disease and related disorders assessed by a door-to-door survey of inhabitants older than 65 years. Arch Neurol 1995;52:1017-22

Tsai CH, Lu CS, Hua MS, Lo WL, Lo SK: Cognitive dysfunction in early onset parkinsonism. Acta Neurol Scand 1994;89: 9-14

Van Bogaert L. Contribution clinique et anatomique à l'étude de la paralysie agitante juvenile primitive. Rev Neurol 1930;2:315-326

Van Dijk PTM, Dippel DWJ, Habbema JDF. Survival of patients with dementia. J Am Geriatr Soc 1991;39:603-610

Vanacore N, Bonifati V, Bellatreccia A, Edito F, Meco G. Mortality rates for Parkinson's disease and parkinsonism in Italy (1969-1987). Neuroepidemiology 1992;11:65-73

Waite LM, Broe GA, Creasey H, Grayson D, Edelbrock D, O'Toole B. Neurological signs, aging, and the neurodegenerative syndromes. Arch Neurol 1996;53:498-502 Wang SJ, Fuh JL, Teng EL, et al. A door-to-door survey of Parkinson's disease in a Chinese population in Kinmen. Arch Neurol 1996 Jan;53:66-71

Warburton JW. Depressive symptoms in Parkinson patients referred for thalamotomy. Neurol Neurosurg Psychiatry 1967;30:368-70 
Wenning GK, Ben Shlomo Y, Magalhaes M, Daniel SE, Quinn NP. Clinical features and natural history of multiple system atrophy. An analysis of 100 cases. Brain $1994 \mathrm{a} ; 117: 835-45$

Wenning GK, Ben-Shlomo Y, Magalhaes M, Daniel SE, Quinn NP.

Clinicopathological study of 35 cases of multiple system atrophy. J Neurol Neurosurg Psychiatry 1995;58:160-6

Wenning GK, Quinn N, Magalhaes M, Mathias C, Daniel SE. "Minimal change" multiple system atrophy. Mov Disord 1994b;9:161-166

Wermuth L, Joensen P, Bunger N, Jeune B. High prevalence of Parkinson's disease in the Faroe Islands. Neurology 1997;49:426-32

Wermuth L, Knudsen L, Boldsen J. A study of cognitive functions in young onset parkinsonism patients. Acta Neurol Scand 1996;93:21-24

World Health Organisation. International classification of impairments, disabilities and handicaps. A manual of classification relating to the consequences of disease. Geneva: World Health Organisation; 1980.

Yokochi M, Narabayashi H, lizuka R, Nagatsu T: Juvenile parkinsonism-Some clinical, pharmacological, and neuropathological aspects. Adv Neurol 1984;40:407-413

Yokochi M. Nosological concept of juvenile parkinsonism with reference to the doparesponsive syndrome. Adv Neurol 1993;60:548-552

Yoshimura N, Yoshimura I, Asada M, et al. Juvenile Parkinson's disease with widespread Lewy bodies in the brain. Acta Neuropathol 1988;77:213-218 Zetusky WJ, Jankovic J, Pirozzolo FJ. The heterogeneity of Parkinson's disease: clinical and prognostic implications. Neurology 1985;35:522-6 Zhang ZX, Roman GC. Worldwide occurrence of Parkinson's disease: an updated review. Neuroepidemiology 1993;12:195-208

Zijlmans JC, Poels PJ, Duysens J, et al. Quantitative gait analysis in patients with vascular parkinsonism. Mov Disord 1996;11:501-8 
Table 1. Study population by age groups and sex

\begin{tabular}{lccc} 
Age $(\mathrm{yrs})$ & Men & Women & Total \\
\hline $\mathbf{0 - 2 9}$ & 22829 & 25438 & 48267 \\
$\mathbf{3 0 - 3 9}$ & 13214 & 12909 & 26123 \\
$\mathbf{4 0 - 4 9}$ & 8815 & 7774 & 16589 \\
$\mathbf{5 0 - 5 9}$ & 6186 & 5737 & 11923 \\
$\mathbf{6 0 - 6 9}$ & 4291 & 4191 & 8482 \\
$\mathbf{7 0 - 7 9}$ & 2810 & 3540 & 6350 \\
$\mathbf{> 8 0}$ & 1266 & 2608 & 3874
\end{tabular}


Table 2. UK Parkinson's Disease Society Brain Bank clinical diagnostic criteria for Parkinson's disease (Gibb, 1988)

\section{Step 1. Diagnosis of parkinsonian syndrome}

Bradykinesia (slowness of initiation of voluntary movement with progressive reduction in speed and amplitude of repetitive actions).

And at least one of the following:

a. muscular rigidity

b. 4-6 Hz rest tremor

c. postural instability not caused by primary visual, vestibular, cerebellar or proprioceptive dysfunction.

\section{Step 2. Exclusion criteria for Parkinson's disease.}

History of repeated strokes with stepwise progression of parkinsonian features

History of repeated head injury

History of definite encephalitis

Oculogyric crisis

Neuroleptic treatment at onset of symptoms

More than one affected relative

Sustained remission

Strictly unilateral features after three years

Supranuclear gaze palsy

Cerebellar signs

Early severe autonomic involvement 
Early severe dementia with disturbance of memory, language and praxis

Babinski sign

Presence of a cerebral tumour or communicating hydrocephalus on CT scan

Negative response to large doses of levodopa (if malabsorption excluded)

MPTP exposure

Step 3. Supportive prospective positive criteria for Parkinson's disease. Three of more required for diagnosis of definite Parkinson's disease.

Unilateral onset

Rest tremor present

Progressive disorder

Persistent asymmetry affecting the side of onset most

Excellent response (70-100\%) to levodopa

Severe levodopa-induced chorea

Levodopa response for 5 years or more

Clinical course of 10 years or more 
Table 3. NINDS-SPSP clinical criteria for the diagnosis of progressive supranuclear palsy (Litvan, 1996)

Mandatory inclusion criteria

Possible Gradually progressive disorder

Onset at age 40 or later

Either vertical (upward or downward gaze) supranuclear palsy* or both slowing of vertical saccades and prominent postural instability with falls in the first year of disease onset

No evidence of other diseases that could explain the foregoing features, as indicated by mandatory exclusion criteria

\section{Mandatory exclusion criteria}

Recent history of encephalitis

Alien limb syndrome, cortical

Sensory deficits, focal frontal or

temporoparietal atrophy

Hallucinations or delusions unrelated to dopaminergic therapy

Cortical dementia of Alzheimer's type

(severe amnesia and aphasia or agnosia, according to NINCDS-ADRA criteria)

Prominent early cerebellar symptoms or prominent, early unexplained dysautonomia (marked hypotension and urinary disturbances)

Severe asymmetric parkinsonian signs

(i.e. bradykinesia)

Neuroradiologic evidence of relevant
Supportive criteria

Symmetric akinesia or

rigidity, proximal more than distal; abnormal neck posture, especially retrocollis

Poor or absent response of parkinsonism to levodopa therapy Early dysphagia and dysarthria; Early onset of cognitive impairment including at least two of the following: apathy, impairment in abstract thought, decreased verbal fluency, utilization or imitation behavior, or frontal release signs 
Probable

Gradually progressive disorder

Onset at age 40 or later

Vertical (upward or downward gaze)

supranuclear palsy* and prominent

postural instability with falls in the

first year of disease onset

No evidence of other diseases that could

explain the foregoing features, as

indicated by mandatory exclusion criteria

Definite

Clinically probable or possible and

histopathologic evidence of typical PSP structural abnormality (i.e. basal ganglia

or brainstem infarcts, lobar atrophy)

Whipple's disease, confirmed by

polymerase chain reaction, if indicated

*Upward gaze is considered abnormal when pursuit or voluntary gaze or both, have a restriction of at least $50 \%$ of the normal range; ${ }^{\#}$ Definite PSP is a clinicopathologic diagnosis 
Table 4. Multiple system atrophy: diagnostic criteria (Quinn, 1989)

\section{SND-type}

(predominantly parkinsonism)

Sporadic adult-onset

non-/poorly levodopa

responsive parkinsonism

Above, 1 plus severe

symptomatic autonomic

failure $^{2}$ or cerebellar signs

or pyramidal signs or

pathological sphincter EMG

$\underline{\text { Post-mortem confirmed }}$

\section{OPCA-type}

(predominantly cerebellar)

possible Sporadic adult-onset cerebellar

syndrome with parkinsonism

probable Sporadic adult-onset cerebellar

syndrome* (with or without

parkinsonism or pyramidal signs),

plus severe symptomatic autonomic

failure $^{2}$ or pathological sphincter EMG

definite Post-mortem confirmed

*without DSM III-R dementia, generalized tendon areflexia, prominent supranuclear palsy for downgaze or other identifiable cause; ${ }^{1}$ moderate or good, but often waning, response to levodopa may occur, in which case multiple atypical features need to be present; ${ }^{2}$ postural syncope and/or marked urinary incontinence or retention not due to other causes. Sporadic: no other case of MSA among first or second degree relatives. Adult onset: onset age 30 years or above. 
Table 5. Diagnostic criteria for corticobasal degeneration (Litvan, 1997a)

Inclusion criteria

1. Chronic progressive course

2. Asymmetric at onset (includes speech, dyspraxia, dysphasia)

3. Presence of higher cortical dysfunction, including either

a) ideomotor apraxia

b) cortical sensory loss or

c) alien limb syndrome

4. Presence of movement disorder including rigid/akinetic syndrome resistant to levodopa and either apraxias a) dystonic limb posturing or

b) spontaneous and reflex focal myoclonus
Exclusion criteria

1. Presence of levodopa responsivity (other than mild worsening on withdrawal)

2. Presence of typical parkinsonian resting tremor

3. Presence of severe autonomic disturbances including symptomatic postural hypotension, urinary or bowel incontinence, and constipation to the point of repeated impaction

4. Presentation with cognitive disturbances other than or speech and language disorders

5. Presence of DSM-IV acceptable dementia while the patient remains ambulatory 


\section{Table 6. Clinical features of patients with probable or possible multiple system atrophy and progressive supranuclear palsy, and of patients with atypical parkinsonism}

Patient Age Age of onset Sex dd Symptom Pre-study Response Additional

(yrs) (yrs) (yrs) at onset diagnosis to l-dopa features

Probable PSP

1

2

$3 \quad 79 \quad 69$

$4 \quad 67 \quad 66$

$76 \quad 69$

$\begin{array}{cc}\text { m } & 4 \\ \text { f } & 1 \\ \text { f } & 10 \\ \text { f } & 1 \\ \text { m } & 7.5\end{array}$

fatigue and
unsteadiness
falls
falls

$\begin{array}{cc}\text { PD } & \text { poor } \\ \text { vascular } p . & \text { poor } \\ & \text { poor }\end{array}$

early frequent falls and hypophonia, SNGP, cognitive impairment, axial>distal akinesia

$\begin{array}{ccc}\text { slowness } & \text { PD } & \text { none } \\ \text { falls } & \text { PD } & \text { poor }\end{array}$

initial falls, symmetric, early dysphagia, mild cognitive impairment, frontalis overactivity, SNGP

axial>>distal bradykinesia, frontalis overactivity, SNGP, visual impairment, staring face, cognitive impairment

incontinence, cognitive impairment, SNGP, axial>distal bradykinesia, initial falls, frontalis overactivity, rapid progression, symmetric

frequent falls, urinary incontinence, visual disturbance, SNGP, severe side effects of medication,

extensor plantar

Possible PSP

slowness

declining

Probable MSA

$$
1
$$

$76 \quad 71$

f 5

unsteadiness

PD

slowness

PD

none

poor

SNGP but no falls, symmetric, mild cognitive impairment, frontalis overactivity, axial>distal bradykinesia, change of personality, urinary incontinence, emotional lability, staring face, extensor plantar

Possible MSA

$$
1
$$

f 2

tremor

$2^{a}$

68

63

m 5

tremor

PD

insufficient dose urinary retention, falls, high-pitched voice

?MSA

poor

severe antecollis, peripheral neuropathy, urinary incontinence and retention, postural hypotension (but also diabetes mellitus)

Atypical parkinsonism

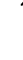

55

f 7

tremor

PD

good

frequent falls (10 per day), extensor plantar, non-specific visual disturbance, predominantly axial features

PD

insufficient dose early falls, urinary incontinence and retention, en-bloc sitting down

$62 \quad 58$

m 4

tremor

PD

good

one fall and

PD

poor

early dysphagia, urinary urgency and frequency, hyperreflexia

slowness

$d d=d i s e a s e$ duration, ${ }^{a}=$ not personally examined, $S N G P=$ vertical supranuclear gaze palsy, $P D=$ Parkinson's disease, $p .=$ parkinsonism 


\section{Progressive supranuclear palsy}

\begin{tabular}{|c|c|c|c|c|c|c|c|c|c|c|c|c|c|c|c|c|c|c|}
\hline \multirow[b]{3}{*}{ Age group } & \multicolumn{5}{|c|}{ Probable and possible cases } & \multicolumn{5}{|c|}{ Only probable cases } & \multirow[b]{2}{*}{ Total } & & \multicolumn{6}{|c|}{ Probable, possible, and atypical ${ }^{1, a}$ cases } \\
\hline & \multicolumn{2}{|l|}{ Men } & \multicolumn{2}{|c|}{ Women } & \multirow{2}{*}{$\begin{array}{c}\text { Total } \\
\mathbf{n} \\
\end{array}$} & \multirow[b]{2}{*}{ rate } & \multicolumn{2}{|l|}{ Men } & \multicolumn{2}{|c|}{ Women } & & & Men & & Won & & Total & \\
\hline & $n$ & rate & $\mathbf{n}$ & rate & & & $\mathbf{n}$ & rate & $\mathrm{n}$ & rate & $\mathbf{n}$ & rate & $\mathbf{n}$ & rate & $\mathbf{n}$ & rate & $\mathbf{n}$ & rate \\
\hline $0-49$ & 0 & 0.0 & 0 & 0.0 & 0 & 0.0 & 0 & 0.0 & 0 & 0.0 & 0 & 0.0 & 0 & 0.0 & 0 & 0.0 & 0 & 0.0 \\
\hline $50-59$ & 1 & 16.2 & 0 & 0.0 & 1 & 8.4 & 1 & 16.2 & 0 & 0.0 & 1 & 8.4 & 1 & 16.2 & 0 & 0.0 & 1 & 8.4 \\
\hline $60-69$ & 0 & 0.0 & 1 & 23.9 & 1 & 11.8 & 0 & 0.0 & 1 & 47.7 & 1 & 11.8 & 1 & 23.3 & 3 & 71.6 & 4 & 47.2 \\
\hline $70-79$ & 1 & 35.6 & 2 & 56.5 & 3 & 47.2 & 1 & 35.6 & 2 & 28.2 & 3 & 47.2 & 1 & 35.6 & 3 & 84.8 & 4 & 63.0 \\
\hline$>80$ & 1 & 79.0 & 0 & 0.0 & 1 & 25.8 & 0 & 0.0 & 0 & 0.0 & 0 & 0.0 & 1 & 79.0 & 0 & 0.0 & 1 & 25.8 \\
\hline Total ${ }^{*}$ & 3 & 5.9 & 3 & 7.1 & 6 & 6.4 & 2 & 4.0 & 3 & 7.3 & 5 & 5.5 & 4 & 8.1 & 6 & 14.3 & 10 & 11.1 \\
\hline
\end{tabular}

Multiple system atrophy

\begin{tabular}{|c|c|c|c|c|c|c|c|c|c|c|c|c|c|c|c|c|c|c|}
\hline \multirow[b]{3}{*}{ Age group } & \multicolumn{5}{|c|}{ Probable and possible cases } & \multicolumn{5}{|c|}{ Only probable cases } & \multirow{2}{*}{ Total } & & \multicolumn{6}{|c|}{ Probable, possible, and atypical ${ }^{1, b}$ cases } \\
\hline & \multicolumn{2}{|l|}{ Men } & \multicolumn{2}{|c|}{ Women } & \multicolumn{2}{|l|}{ Total } & \multicolumn{2}{|l|}{ Men } & \multicolumn{2}{|c|}{ Women } & & & Men & & Wom & & Total & \\
\hline & $\mathbf{n}$ & rate & $\mathbf{n}$ & rate & $\mathbf{n}$ & rate & $\mathbf{n}$ & rate & $\mathbf{n}$ & rate & $\mathbf{n}$ & rate & $\mathbf{n}$ & rate & $\mathbf{n}$ & rate & $\mathbf{n}$ & rate \\
\hline $0-49$ & 0 & 0.0 & 0 & 0.0 & 0 & 0.0 & 0 & 0.0 & 0 & 0.0 & 0 & 0.0 & 0 & 0.0 & 0 & 0.0 & 0 & 0.00 \\
\hline $50-59$ & 0 & 0.0 & 0 & 0.0 & 0 & 0.0 & 0 & 0.0 & 0 & 0.0 & 0 & 0.0 & 0 & 0.0 & 0 & 0.0 & 0 & 0.00 \\
\hline $60-69$ & 1 & 23.3 & 0 & 0.0 & 1 & 11.8 & 0 & 0.0 & 0 & 0.0 & 0 & 0.0 & 2 & 46.6 & 2 & 47.7 & 4 & 47.20 \\
\hline $70-79$ & 0 & 0.0 & 2 & 56.5 & 2 & 31.5 & 0 & 0.0 & 2 & 56.5 & 2 & 31.5 & 0 & 0.0 & 3 & 84.7 & 3 & 47.24 \\
\hline$>80$ & 0 & 0.0 & 1 & 38.3 & 1 & 25.8 & 0 & 0.0 & 0 & 0.0 & 0 & 0.0 & 0 & 0.0 & 1 & 38.3 & 1 & 25.81 \\
\hline Total* & 1 & 2.3 & 3 & 6.6 & 4 & 4.4 & 0 & 0.0 & 2 & 4.6 & 2 & 2.2 & 2 & 4.5 & 6 & 13.8 & 8 & 9.03 \\
\hline
\end{tabular}

* Total rates have been age-adjusted to the European population; 1 patients with atypical parkinsonism not fulfilling criteria for progressive supranuclear palsy or multiple system atrophy, ${ }^{a}$ if all atypical cases have PSP, ${ }^{b}$ if all atypical cases have MSA; $n=n u m b e r$ of cases 
Table 8. Patient characteristics of all patients with parkinsonism

Probable and Atypical Drug-induced Parkinsonism Vascular possible IPD parkinsonism parkinsonism following dementia pseudoparkinsonism

n 156

$\mathrm{M}: \mathrm{F}$

Median age (yrs)

Age range (yrs)

$82: 74$

14

43

$24: 19$

$71.5 \quad 67$

$34-94$

$57-87$

$42-97$

17

$11: 6$

78

$\begin{array}{cc}4.1 & 78 \\ 77 & 63-97\end{array}$


Table 9. Age and sex specific prevalence rates of Parkinson's disease and parkinsonism

\begin{tabular}{|c|c|c|c|c|c|c|c|c|c|c|c|c|}
\hline & \multicolumn{3}{|c|}{ Parkinson's disease $^{a}$} & \multicolumn{9}{|c|}{ All types of parkinsonism } \\
\hline & Men & & Women & & Total & & Men & & Women & & Total & \\
\hline Age & $\mathrm{n}$ & prevalence & $\mathrm{n}$ & prevalence & $\mathrm{n}$ & prevalence & $\mathrm{n}$ & prevalence & $n$ & prevalence & $\mathrm{n}$ & prevalence \\
\hline $0-29$ & 0 & 0 & 0 & 0 & 0 & 0 & 0 & 0 & 0 & 0 & 0 & 0 \\
\hline $40-49$ & 2 & 23 & 0 & 0 & 2 & 12 & 45 & 45 & 0 & 0 & 4 & 24 \\
\hline 50-59 & 9 & 145 & 4 & 70 & 13 & 109 & 162 & 162 & 7 & 122 & 17 & 143 \\
\hline $60-69$ & 19 & 443 & 10 & 239 & 29 & 342 & 746 & 746 & 21 & 501 & 53 & 625 \\
\hline$>80$ & 21 & 1659 & 28 & 1074 & 49 & 1265 & 5093 & 2370 & 38 & 1457 & 68 & 1755 \\
\hline Crude rate & 82 & 138 & 74 & 119 & 156 & 128 & 126 & 212 & 109 & 175 & 235 & 193 \\
\hline $\begin{array}{l}\text { Age-adjusted } \\
\text { rate }^{\text {b }}\end{array}$ & & 171 & & 164 & & 168 & & 265 & & 241 & & 154 \\
\hline
\end{tabular}

prevalence=prevalence per $100,000,{ }^{2}=$ including probable and possible Parkinson's disease

$b=$ adjusted to the 1997 UK population 
Table 10. Age-specific prevalence rates for atypical, drug-induced and post dementia parkinsonism

\begin{tabular}{|c|c|c|c|c|c|c|c|c|}
\hline \multirow[b]{2}{*}{ Age } & \multicolumn{2}{|c|}{ Atypical $^{\mathrm{a}}$} & \multicolumn{2}{|c|}{ Vascular } & \multicolumn{2}{|c|}{ Drug-induced } & \multicolumn{2}{|c|}{ Post-dementia } \\
\hline & $\mathbf{n}$ & prevalence & $n$ & prevalence & $n$ & prevalence & $\mathrm{n}$ & prevalence \\
\hline $0-29$ & 0 & 0 & 0 & 0 & 0 & 0 & 0 & 0 \\
\hline $30-39$ & 0 & 0 & 0 & 0 & 0 & 0 & 0 & 0 \\
\hline $40-49$ & 0 & 0 & 0 & 0 & 2 & 12 & 0 & 0 \\
\hline $50-59$ & 1 & 8 & 0 & 0 & 3 & 25 & 0 & 0 \\
\hline $60-69$ & 5 & 59 & 1 & 12 & 17 & 200 & 1 & 12 \\
\hline $70-79$ & 6 & 94 & 9 & 142 & 13 & 205 & 2 & 31 \\
\hline$>80$ & 2 & 52 & 7 & 181 & 8 & 207 & 2 & 52 \\
\hline Crude rate & 14 & 12 & 17 & 14 & 43 & 35 & 5 & 4 \\
\hline $\begin{array}{l}\text { Age-adjust } \\
\text { rate }^{\mathrm{b}}\end{array}$ & & 15 & & 19 & & 46 & & 6 \\
\hline
\end{tabular}

rate

prevalence=prevalence per 100,$000 ;{ }^{a}$ including MSA, PSP and atypical parkinsonism

${ }^{b}=$ adjusted to the 1997 UK population 
Table 11. Breakdown of parkinsonism in different subtypes

\begin{tabular}{cccccccc} 
& \multicolumn{2}{c}{ Total } & \multicolumn{3}{c}{ Men } & \multicolumn{3}{c}{ Women } \\
& & $\mathrm{n}$ & $\%$ & $\mathrm{n}$ & $\%$ & $\mathrm{n}$ & $\%$ \\
\hline Primary degenerative parkinsonism & & 170 & 72.3 & 87 & 69.0 & 83 & 76.1 \\
Parkinson's disease & & 156 & 66.4 & 82 & 65.1 & 74 & 67.9 \\
& probable & 152 & 64.7 & 79 & 62.7 & 73 & 67.0 \\
& possible & 4 & 1.7 & 3 & 2.4 & 1 & 0.9 \\
Atypical parkinsonism & & 14 & 6.0 & 5 & 4.0 & 9 & 8.3 \\
MSA & & 4 & 1.7 & 1 & 0.8 & 3 & 2.8 \\
& probable & 2 & 0.9 & 0 & 0.0 & 2 & 1.8 \\
PSP & possible & 2 & 0.9 & 1 & 0.8 & 1 & 0.9 \\
& & 6 & 2.6 & 3 & 2.4 & 3 & 2.8 \\
& probable & 5 & 2.1 & 2 & 1.6 & 3 & 2.8 \\
Atypical, not classifiable & possible & 1 & 0.4 & 1 & 0.8 & 0 & 0.0 \\
Parkinsonism due to other causes & & 4 & 1.7 & 1 & 0.8 & 3 & 2.8 \\
Drug-induced & & 65 & 27.7 & 39 & 31.0 & 26 & 23.9 \\
Vascular & & 43 & 18.3 & 24 & 19.0 & 19 & 17.4 \\
Dementia & & 17 & 7.2 & 11 & 8.7 & 6 & 5.5 \\
& & 5 & 2.1 & 4 & 3.2 & 1 & 0.9
\end{tabular}


Table 12. Initial and final diagnoses of patients seen in this population-based study $(n=202)$

Initial diagnosis

Percentage Final diagnosis

n Percentage

Parkinson's disease

$131 \quad 64.9$

Probable Parkinson's disease

Possible Parkinson's disease

124

61.4

Atypical parkinsonism

1

2

Non-parkinsonian trem

0.5

Multiple system atrophy

Non-parkinsonian tremor

27.7 Progressive supranuclear palsy

$4 \quad 2.0$

Other patients on antiparkinsonian medication

Diagnostic referral

5.0 Non-parkinsonian tremor

$3 \quad 1.5$

10

1.0

Vascular parkinsonism

$6 \quad 3.0$

$46 \quad 22.8$


Table 13. Patients with an initial diagnosis of Parkinson's disease $(n=131)$

\begin{tabular}{lccccc} 
Final diagnosis & $\mathrm{n}$ & $\%$ & $\mathrm{M}: \mathrm{F}$ & Age & Seen by neurologist (\%) \\
\hline Probable Parkinson's disease & 109 & 83 & $59: 51$ & 71.6 & 78 \\
Possible Parkinson's disease & 2 & 1.5 & $1: 1$ & 81.5 & 100 \\
Multiple system atrophy & 3 & 2.3 & $0: 3$ & 78.3 & 0 \\
Progressive supranuclear palsy & 4 & 3.1 & $3: 1$ & 71.8 & 50 \\
Non-parkinsonian tremor & 4 & 3.1 & $3: 1$ & 65.5 & 67 \\
Vascular parkinsonism & 6 & 4.6 & $4: 2$ & 82.5 & 50 \\
Other & 3 & 2.3 & $1: 2$ & 84.7 & 0
\end{tabular}


Table 14. Patients with an final diagnosis of Parkinson's disease $(n=124$, incl. patients with atypical parkinsonism not

\begin{tabular}{lccccc} 
Initial diagnosis & $\mathrm{n}$ & $\%$ & $\mathrm{M}: \mathrm{F}$ & $\begin{array}{c}\text { Mean Age } \\
(\mathrm{yrs})\end{array}$ & $\begin{array}{c}\text { Seen by neurologist } \\
(\%)\end{array}$ \\
\hline Parkinson's disease & 109 & 85.2 & $56: 53$ & 72 & 74 \\
Atypical parkinsonism & 1 & 0.8 & $1: 0$ & 55 & 100 \\
Vascular parkinsonism & 1 & 0.8 & $0: 1$ & 69 & 0 \\
Non-parkinsonian tremor & 9 & 7.0 & $5: 4$ & 74 & 0 \\
Patients on antiparkinsonian medication & 2 & 1.6 & $0: 2$ & 84.5 & 0 \\
Referred for diagnosis & 2 & 1.6 & $2: 0$ & 82.5 & 0
\end{tabular}


Table 15. Median disease duration (SD) by Hoehn and Yahr stage

$\begin{array}{lccccc} & \text { HY stage I } & \text { HY stage II } & \text { HY stage III } & \text { HY stage IV } & \text { HY stage V } \\ \text { Hoehn and Yahr }{ }^{1,2} & 3.0(7.1) & 6.0(6.9) & 7.0(6.3) & 9.0(7.2) & 14.0(3.4) \\ \text { Marttila and Rinne } & \text { not available } & 2.9(0.2) & 5.5(0.3) & 7.5(0.6) & 9.7(1.0) \\ \text { All patients in this study } & 3.0(2.1) & 5.2(4.2) & 5.8(5.2) & 6.7(3.5) & 5.7(4.6) \\ \text { Patients on levodopa in this study } & 4.0(2.1) & 6.0(4.7) & 7.0(4.6) & 7.4(2.9) & 5.7(4.6)\end{array}$

${ }^{1}$ Derived from Poewe and Wenning (Poewe) ${ }^{2}$ Before treatment with levodopa was available (Hoehn), ${ }^{3}$ patients on levodopa (Martilla), mean values (SD) 
Table 16. Prediction of rate of progression ${ }^{1}$

$\begin{array}{lcccc} & \text { R2 } & \text { R2 change } & \text { Adjusted R2 } & \text { F-change } \\ \text { Dementia }^{2} & & 0.17 & & 9 \\ \text { Treatment with levodopa } & & 0.08 & & 4.62 \\ \text { Onset age } & 0.32 & 0.07 & 0.27 & 4.62\end{array}$

${ }^{1}$ Calculated from Hoehn and Yahr stage divided by disease duration in years; ${ }^{2}$ Mini mental state score 24 out of 30 or less 
Table 17. Characteristics of patients on levodopa $(n=87)$

\begin{tabular}{|c|c|c|}
\hline & Mean & SD \\
\hline Age (yrs) & 71.3 & 11.6 \\
\hline Disease duration (yrs) & 6.8 & 4.3 \\
\hline Age at onset (yrs) & 64.5 & 7.3 \\
\hline Duration of treatment with levodopa (yrs) & 5.2 & 4.4 \\
\hline Dose of levodopa (mgs/day) & 423.3 & 227.6 \\
\hline Hoehn and Yahr stage & 2.4 & 0.8 \\
\hline Schwab and England score & 81.3 & 15.1 \\
\hline PDQ 39 & 28.8 & 18.3 \\
\hline \multicolumn{3}{|l|}{ Percent of patients who are } \\
\hline Male & 47.1 & \\
\hline On amantadine & 5 & \\
\hline On selegiline & 32.5 & \\
\hline On an anticholinergic & 12.5 & \\
\hline On a dopamine agonist & 13.8 & \\
\hline
\end{tabular}


Table 18. Percent of patients with motor fluctuations and dyskinesias by disease duration

Disease duration

Motor fluctuations

Overall sample

Patients on levodopa
Patients with a good ${ }^{1}$ response to levodopa

$<5$ years $5-10$ years $>10$ years

Overall sample

Dyskinesias

Patients on levodopa

Patients with a good ${ }^{1}$ response to levodopa

61.5

$\begin{array}{lll}10.5 & 40 & 66.7\end{array}$

$22.2 \quad 50 \quad 72.7$

$\begin{array}{lll}2.9 & 13 & 46.3\end{array}$

$5 \quad 15 \quad 58.3$

${ }^{1}$ response subjectively $>50 \%$ 
Table 19. Differences between patients with and without motor fluctuations and dyskinesias (mean/SD)

\begin{tabular}{|c|c|c|c|c|c|c|}
\hline & \multicolumn{3}{|c|}{ Motor fluctuations } & \multicolumn{2}{|l|}{ Dyskinesias } & \multirow[b]{2}{*}{ p-value $<$} \\
\hline & With & Without & p-value $<$ & With & Without & \\
\hline Age, yrs & $68.8 / 10.2$ & $73.3 / 12.2$ & $\mathrm{~ns}$ & $70.0 / 11.3$ & $71.8 / 11.7$ & ns \\
\hline Disease duration, yrs & $9.0 / 5.2$ & $5.2 / 2.6$ & 0.0001 & $8.8 / 5.8$ & $5.9 / 3.5$ & $0.01^{1}$ \\
\hline Time since diagnosis, yrs & $7.2 / 4.7$ & 4.3/4.1 & $0.05^{1}$ & $7.4 / 3.8$ & $5.0 / 4.8$ & ns \\
\hline Treatment with levodopa, yrs & $7.4 / 5.4$ & $3.5 / 2.6$ & 0.0001 & $7.5 / 6.0$ & $4.2 / 3.4$ & 0.005 \\
\hline Time from onset to initiation of levodopa & $0.5 / 0.8$ & $1.5 / 1.5$ & ns & $0.5 / 3.8$ & $1.7 / 2.3$ & ns \\
\hline Levodopa dose, mgs/day & $542.3 / 224.1$ & $340.4 / 191.8$ & 0.0001 & $515.8 / 221.2$ & $393.9 / 228.9$ & $0.05^{1}$ \\
\hline Hoehn and Yahr stage & $2.6 / 0.7$ & $2.2 / 0.7$ & $0.01^{1}$ & $2.7 / 0.7$ & $2.3 / 0.7$ & $0.05^{1}$ \\
\hline $\begin{array}{l}\text { Schwab and England score } \\
\text { Percent of patients with }\end{array}$ & $73.8 / 18.3$ & $86.2 / 10.1$ & 0.005 & $72.6 / 17.6$ & $84.7 / 12.9$ & 0.001 \\
\hline Levodopa dose $>300 \mathrm{mgs} /$ day & 79.4 & 20.6 & 0.0001 & 73.7 & 45.9 & $0.01^{1}$ \\
\hline Good response to levodopa & 100 & 56.3 & 0.001 & 100 & 65 & $0.05^{1}$ \\
\hline Family history of parkinsonism & 39.3 & 11.1 & $0.05^{1}$ & 43 & 20.2 & ns \\
\hline Akinetic-rigid subtype & 94.7 & 53.5 & $0.05^{1}$ & 90 & 71.2 & ns \\
\hline Falls & 78.9 & 58.3 & ns & 100 & 56.8 & $0.01^{1}$ \\
\hline
\end{tabular}


Table 20. Factors associated with significantly increased depression scores

\section{Beck Depression Inventory}

Mean score (SD) p-value<

\begin{tabular}{|c|c|c|c|}
\hline Change in health compared to previous year ${ }^{1}$ & Worsening & $18.6(12.7)$ & 0.001 \\
\hline \multicolumn{2}{|c|}{ Improvement or no change } & $9.7(8.3)$ & \\
\hline \multirow{2}{*}{ Falls $^{1}$} & Present & $14.5(11.9)$ & 0.01 \\
\hline & Absent & $8.4(6.5)$ & \\
\hline \multirow[t]{2}{*}{ Postural instability } & Present & $13.5(11.9)$ & $0.05^{2}$ \\
\hline & Absent & $7.9(5.4)$ & \\
\hline \multirow[t]{2}{*}{ Akinetic-rigid subtype } & Present & $14.0(12.3)$ & $0.05^{2}$ \\
\hline & Absent & $8.0(6.0)$ & \\
\hline \multirow[t]{2}{*}{ Impaired dexterity ${ }^{1}$} & Present & $13.5(10.3)$ & $0.05^{2}$ \\
\hline & Absent & $9.6(10.7)$ & \\
\hline \multirow[t]{2}{*}{ Stiffness $^{1}$} & Present & $14.4(11.8)$ & $0.05^{2}$ \\
\hline & Absent & $9.9(8.3)$ & \\
\hline \multirow[t]{2}{*}{ Minimental score $<25$} & Present & $16.5(12.2)$ & $0.05^{2}$ \\
\hline & Absent & $9.2(6.4)$ & \\
\hline \multirow[t]{2}{*}{ Hallucinations $^{1}$} & Present & $15.1(8.6)$ & $0.05^{2}$ \\
\hline & Absent & $9.0(6.6)$ & \\
\hline \multirow[t]{2}{*}{ Speech impairment ${ }^{1}$} & Present & $13.9(10.6)$ & $0.05^{2}$ \\
\hline & Absent & $10.6(10.8)$ & \\
\hline
\end{tabular}

${ }^{1}$ self-reported, ${ }^{2}$ marginally significant 
Table 21. Comparison of the depressed (BDI>17) and non-depressed patients on measures of impairment, disability and handicap (Standard deviations are given in parentheses)

\section{Level Variable}

Impairment Postural instability* (mean; SD)

$\bar{\alpha}$
Depressed

\begin{tabular}{ccc} 
Depressed & Non-depressed & p-value< \\
1.9 & 1.1 & 0.001 \\
25.7 & 27.9 & 0.01 \\
20 & 61.5 & 0.005 \\
$71.4(22.4)$ & $83.6(14.0)$ & 0.002 \\
$48(29.0)$ & $79(16.4)$ & 0.0001 \\
$52.9(23.5)$ & $25.3(18.3)$ & 0.00001 \\
$50.1(26.9)$ & $32.6(19.0)$ & 0.0005 \\
$35.4(28.0)$ & $15.3(17.1)$ & 0.0001 \\
$46.0(25.8)$ & $23.2(16.6)$ & 0.0005 \\
$29.4(26.1)$ & $10.2(16.3)$ & 0.001 \\
\hline $51.1(29.4)$ & $33.1(26.6)$ & 0.003 \\
$32.3(29.7)$ & $19.0(25.4)$ & 0.004 \\
$63.5(31.1)$ & $45.5(32.0)$ & 0.004 \\
\hline
\end{tabular}

Cognition
Bodily pain
Communication
Emotional well-being
Social support
Activities of daily living
Stigma
Mobility

"measured on the Unified Parkinson's Disease Rating Scale 
Table 22. Results of stepwise multiple regression analysis of BDI scores

Independent variable

Standardized Regression $R$ square $R$ square Adjusted t value Significance

Analysis 2a Disability Observer-rated disability (SE)

Cognition (PDQ-39)

Stigma (PDQ-39)

Observer-rated disability (SE)

Recent deterioration
Coefficient

0.35

Recent deterioration

Analysis $2 b$

Analysis 3

Handicap

Patient-rated disability (SE)

Cognition (PDQ-39)

Stigma (PDQ-39)

change $\mathrm{R}$ square

$(p<)$

Analysis 4

Overall

3.48

$\begin{array}{llll}0.11 & 0.28 & 3.39 & 0.001\end{array}$

0.51

8.35
0.34

0.35
0.34
-0.58
-0.72
0.54
0.31
0.36
0.26
-0.25

0.2

36
0.19

0.3

0.34

0.52

0.47

0.54

0.08

0.47

0.08

0.05

0.62

0.04

0.52
0.001

$\begin{array}{llll}0.33 & 0.34 & 6.1 & 0.0001\end{array}$

0.0001

$6.47 \quad 0.0001$

$\begin{array}{lll}0.53 \quad 3.39 & 0.0001\end{array}$

$3.75 \quad 0.0001$

$3.03 \quad 0.003$

$2.82 \quad 0.006$

$\mathrm{S}=$ Schwab and England scale

Multiple $\mathrm{R}=0.79, \mathrm{R} 2=0.62$, adjusted $\mathrm{R} 2=0.6$ 


\section{Table 23. Comparison of early vs late onset PD}

\begin{tabular}{|c|c|c|c|c|c|}
\hline \multirow[t]{2}{*}{ Variable } & \multicolumn{2}{|l|}{$\begin{array}{l}\text { Early onset } \\
\text { (<67 years) }\end{array}$} & \multicolumn{2}{|c|}{$\begin{array}{l}\text { Late onset } \\
\text { (>67 years) }\end{array}$} & \multirow[b]{2}{*}{$p$-value $<$} \\
\hline & Mean & SD & Mean & SD & \\
\hline Age (yrs) & 50.1 & 11.1 & 81.4 & 7.1 & 0.0001 \\
\hline Age at onset (yrs) & 41.1 & 9.7 & 79 & 9.7 & 0.0001 \\
\hline Duration since symptom onset (yrs) & 9 & 4.5 & 2.4 & 1.9 & 0.005 \\
\hline Progression rate ${ }^{1}$ & 0.31 & 0.14 & 1.09 & 1.51 & $0.01^{2}$ \\
\hline Fluctuations (\%) & 46.2 & & 16.7 & & $0.05^{2}$ \\
\hline
\end{tabular}

${ }^{1}$ Hoehn and Yahr stage divided by disease duration in years, ${ }^{2}$ marginally significant 
Table 24. Comparison of akinetic-rigid vs tremor-dominant type

\begin{tabular}{lccccc} 
Variable & \multicolumn{3}{c}{ Akinetic-rigid type } & Tremor-dominant type \\
& Mean & SD & Mean & SD & p-value< \\
\hline Duration since symptom onset (yrs) & 6.4 & 4.1 & 4.3 & 2.3 & $0.05^{1}$ \\
Hoehn andYahr stage & 2.5 & 0.9 & 1.9 & 0.6 & 0.005 \\
Schwab and England score & 79.6 & 15.5 & 89.3 & 11 & 0.0001 \\
Depression score & 13.7 & 12.6 & 7.2 & 5.5 & $0.01^{1}$ \\
Quality of life & 33.5 & 20.8 & 21.5 & 14.9 & $0.05^{1}$ \\
Progression rate & 0.74 & 1.22 & 0.63 & 0.65 & $0.05^{1}$ \\
Onset with tremor (\%) & 20 & & 51 & & $0.05^{1}$ \\
Fluctuations (\%) & 8.3 & & 48.6 & & $0.05^{1}$ \\
Postural instability (\%) & 79.5 & & 50 & & $0.05^{1}$ \\
Freezing (\%) & 41.2 & & 77.6 & & $0.01^{1}$
\end{tabular}


Table 25. Comparison of patients with slow vs those with fast progression

\begin{tabular}{lccccc} 
Variable & \multicolumn{3}{c}{ Slow progression } & Fast progression & \\
& Mean & SD & Mean & SD & p-value< \\
\hline Age (yrs) & 70.5 & 11.6 & 78.6 & 9.8 & $0.05^{4}$ \\
Age at onset (yrs) & 62.7 & 13.7 & 76.7 & 6.7 & 0.0001 \\
Duration since symptom onset (yrs) & 7.8 & 4.7 & 1.9 & 0.9 & 0.0001 \\
Treatment duration (yrs) & 5.8 & 5 & 1.1 & 0.8 & 0.0001 \\
Time from onset to treatment (yrs) & 2 & 0.8 & 2.6 & 0.7 & 0.001 \\
Hoehn and Yahr stage & 2.3 & 0.7 & 2.7 & 0.8 & $0.05^{4}$ \\
Schwab and England score $_{\text {Axial score }}{ }^{1}$ & 83.5 & 14.4 & 77.2 & 16.4 & $0.05^{4}$ \\
Progression rate $^{2}$ & 5 & 3.2 & 7.1 & 4 & $0.05^{4}$ \\
Dementia $^{3}$ (\%) & 0.38 & 0.17 & 1.88 & 1.5 & 0.0001
\end{tabular}

${ }^{1}$ from UPDRS items $27-30,{ }^{2}$ Hoehn and Yahr stage divided by disease duration in years, ${ }^{3}$ mini mental score 24 out of 30 or less, ${ }^{4}$ marginally significant 


\section{Table 26. Results of Cluster analysis to define subtypes of Parkinson's disease}

\begin{tabular}{|c|c|c|c|c|}
\hline Variable & Group 1 & Group 2 & Group 3 & F-value \\
\hline Age at onset (yrs) & 71.7 & 77.4 & 61.3 & 16.3 \\
\hline Duration since symptom onset (yrs) & 6.5 & 4 & 8.1 & 5.1 \\
\hline Age (yrs) & 78.2 & 81.4 & 69.4 & 15.0 \\
\hline Progression rate ${ }^{1}$ & 0.57 & 1.19 & 0.36 & 5.9 \\
\hline Hoehn and Yahr stage & 2.2 & 2.7 & 2.7 & 4.8 \\
\hline Schwab and England score & 86.1 & 79.3 & 71 & 7.4 \\
\hline Duration of treatment with levodopa (yrs) & 5.1 & 2.9 & 6.9 & 3.4 \\
\hline Latency form onset to initiation of levodopa & 1.9 & 1.3 & 1.8 & $.6^{\mathrm{a}}$ \\
\hline Axial score ${ }^{2}$ & 5.1 & 6.5 & 6.2 & $1.5^{\mathrm{a}}$ \\
\hline Quality of life ${ }^{3}$ & 24.5 & 39.8 & 34.5 & 4.5 \\
\hline Mini mental score ${ }^{4}$ & 27.6 & 23.4 & 27.7 & 7.8 \\
\hline Dose of levodopa (mg) & 428.6 & 134.8 & 870 & 150.5 \\
\hline
\end{tabular}

${ }^{1}$ Hoehn and Yahr stage divided by disease duration in years; ${ }^{2}$ derived from UPRDS items 27 to $30 ;{ }^{3} \mathrm{PDQL}$ (Peto); all p<0.01 except ${ }^{\mathrm{a}} \mathrm{ns}$ 
Table 27. Characteristics of patients with Young Onset Parkinson's disease (YOPD)

\begin{tabular}{|c|c|c|c|}
\hline & & $\begin{array}{c}J P \\
(n=10) \\
\end{array}$ & $\begin{array}{c}\text { YOPD } \\
(n=139)\end{array}$ \\
\hline $\bar{M}: F$ & & $8: 2$ & $88: 51$ \\
\hline Age in yrs ${ }^{1}$ (range) & & $41(10-62)$ & $51(33-78)$ \\
\hline Disease duration in $\mathrm{yrs}^{1}$ (range) & & $24(5-45)$ & $18(2-43)$ \\
\hline Onset age ${ }^{1}$ in yrs (range) & & $17(5-19)$ & $34(22-39)$ \\
\hline $\mathrm{FH}(\%)$ & & $5(50 \%)$ & $22(16 \%)$ \\
\hline \multirow[t]{3}{*}{ Onset symptom } & dystonia & $60 \%$ & $14 \%$ \\
\hline & tremor & $50 \%$ & $52 \%$ \\
\hline & akinetic-rigid & $20 \%$ & $69 \%$ \\
\hline \multirow[t]{3}{*}{ Initial response to levodopa } & excellent & $78 \%$ & $81 \%$ \\
\hline & good & $11 \%$ & $15 \%$ \\
\hline & poor or none & $11 \%$ & $4 \%$ \\
\hline
\end{tabular}

$\mathrm{JP}=$ =juvenile parkinsonism; YOPD=young onset Parkinson's disease; $\mathrm{FH}=$ family history

(at least one first- or second degree relative affected); ${ }^{1}$ median 
Table 28. Clinical differences at disease onset between YOPD patients who later became demented and those who had not become demented

\begin{tabular}{|c|c|c|c|c|}
\hline & & $\begin{array}{c}\text { Demented } \\
(n=19)\end{array}$ & $\begin{array}{c}\text { Not demented } \\
(n=79)\end{array}$ & $\begin{array}{c}\text { Statistical } \\
\text { significance }\end{array}$ \\
\hline Median cognitive score (range) & & $24.4(15-30)$ & $35.1(31-41)$ & $p<0.001^{1}$ \\
\hline $\operatorname{Sex}(M: F)$ & & $7: 12$ & $58: 21$ & $p<0.01^{2}$ \\
\hline Positive family history for parkinsonism ${ }^{3}$ & & $4(22.2 \%)$ & $10(13.0 \%)$ & $n s^{2}$ \\
\hline \multirow[t]{3}{*}{ Symptom present at onset } & dystonia & $12 \%$ & $13 \%$ & $n s^{2}$ \\
\hline & tremor & $56 \%$ & $47 \%$ & \\
\hline & akinetic-rigid & $78 \%$ & $65 \%$ & \\
\hline \multirow[t]{3}{*}{ Initial response to levodopa } & excellent & $80 \%$ & $82 \%$ & $\mathrm{~ns}^{2}$ \\
\hline & good & $20 \%$ & $16 \%$ & \\
\hline & none or poor & $0 \%$ & $2 \%$ & \\
\hline
\end{tabular}

YOPD=young onset Parkinson's disease; ${ }^{1}$ t-test for independent samples; ${ }^{2}$ useful prospective predictive variables at disease onset in the discriminant analysis: ${ }^{3}$ two-tailed Chi-square/Fisher's exact test 
Table 29. Clinical differences at latest assessment between demented and nondemented YOPD-patients

\begin{tabular}{lccc} 
& Demented & Not demented & $\begin{array}{c}\text { Statistical } \\
\text { significance }\end{array}$ \\
\hline Mean age in years (range) & $57.8(41-78)$ & $48.8(33-68)$ & $\mathrm{p}<0.001^{1}$ \\
Mean disease duration in years (range) & $22.3(8-41)$ & $15.8(2-43)$ & $\mathrm{p}<0.01^{1}$ \\
No of patients with psychiatric side effects (\%) & $15(83.3)$ & $29(38.2)$ & $\mathrm{p}<0.001^{3}$ \\
Patients with falls (\%) & $12(70.6)$ & $27(37.0)$ & $\mathrm{p}<0.05^{3}$ \\
Patients with freezing (\%) & $15(88.2)$ & $49(68.1)$ & $\mathrm{ns}$ \\
S\&E score in On (range) & $65.7(40-90)$ & $81.2(10--100)$ & $\mathrm{p}<0.01^{1}$ \\
S\&E in Off (range) & $39.1(10-80)$ & $61.1(20-100)$ & $\mathrm{p}<0.01^{1}$
\end{tabular}

YOPD=young onset Parkinson's disease; S\&E Schwab and England rating scale; ${ }^{1}$ t-test for independent samples;

${ }^{2}$ useful prospective variables during the disease course in the discriminant analysis; 3 Chi-square/Fisher's exact test 
Table 30a. Classification of demented patients by female sex and positive family history of parkinsonism

\begin{tabular}{lccc} 
& \multicolumn{2}{c}{ Actual cognitive status } \\
& & demented & non-demented \\
\hline Predicted cognitive status & demented & $12(67 \%)(\mathrm{a})$ & $21(27.3 \%)(\mathrm{b})$ \\
& non-demented & $6(33 \%)(\mathrm{c})$ & $56(73 \%)$ (d)
\end{tabular}

Overall prediction: $72 \%$

Table 30b. Classification of demented patients by female sex, positive family history of parkinsonism, and psychiatric side effects of treatment

Actual cognitive status

demented non-demented

$\begin{array}{cccc}\text { Predicted cognitive status } & \text { demented } & 12(71 \%)(\mathrm{a}) & 12(16 \%)(\mathrm{b}) \\ & \text { non-demented } & 5(29 \%) \text { (c) } & 63(84 \%)(\mathrm{d})\end{array}$

Overall prediction: $82 \%$ 
Table 31. Correct classification of dementia in YOPD

Definition

Sensitivity $=(a / a+c)$

Specificity $=(d / b+d)$

Positive predictive value $=(a / a+b)$
The proportion of demented patients that were correctly predicted to be demented

The proportion of nondemented patients that were correctly predicted to be nondemented

The proportion of those predicted

to be demented that are demented

Negative predictive value $=(d / c+d)$

The proportion of those predicted not

to be demented that are nondemented
Classification by female

sex and family history $66.7 \%$

$72.7 \%$

$36.4 \%$

$90.3 \%$
Classification by female

sex, family history and psychiatric side effects $70.6 \%$

$84.0 \%$

$50.0 \%$

$92.6 \%$

YOPD=young onset Parkinson's disease 
Table 32. Patient characteristics. Standard deviations are given in parentheses.

\begin{tabular}{lccc} 
& & $\mathrm{n}$ & $\%$ \\
\hline Hoehn and Yahr stage & $\mathrm{I}$ & 11 & 11.3 \\
& $\mathrm{I} .5$ & 9 & 9.3 \\
& $\mathrm{II}$ & 22 & 22.7 \\
& $\mathrm{II.5}$ & 29 & 29.9 \\
& $\mathrm{III}$ & 19 & 19.6 \\
& $\mathrm{IV}$ & 3 & 3.1 \\
Mean age (yrs) & $\mathrm{V}$ & 4 & 4.1 \\
Mean disease duration (yrs) & $73.0(11.3)$ & & \\
Sex (M:F) & $5.8(4.9)$ & & \\
Mean age of onset (yrs) & $50: 47$ & & \\
Mean UPDRS score & $67.6(12.5)$ & & \\
Mean BDI score & $22.7(11.3)$ & & \\
Mean mini mental score & $11.5(10.6)$ & & \\
& $27.5(3.6)$ &
\end{tabular}


Table 33. Spearman correlations coefficients of different quality of life measures with clinical scales and disease duration

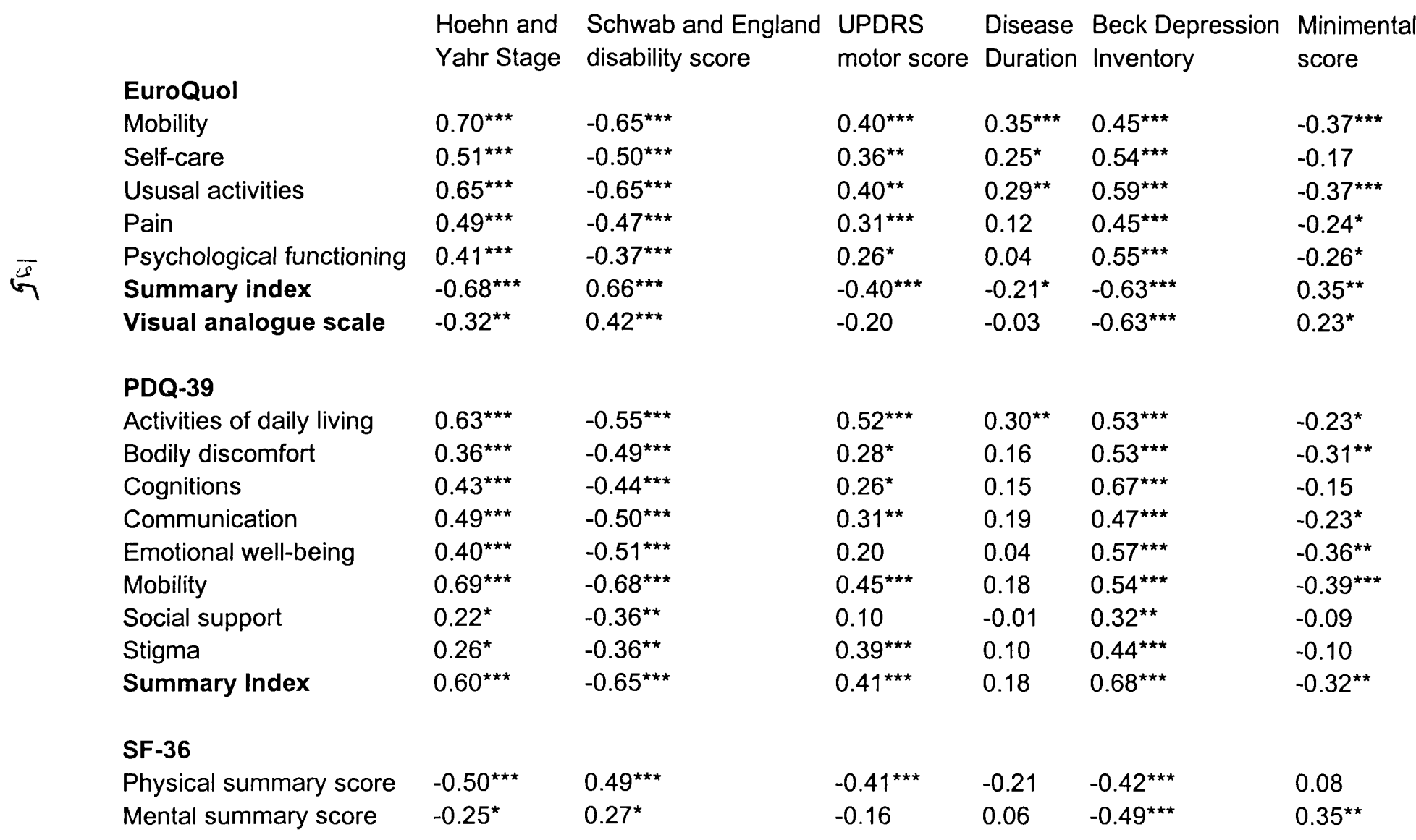

UPDRS=Unified Parkinson's Disease Rating Scale; ${ }^{*} p<0.05,{ }^{* \star} p<0.01,{ }^{* \star *} p<0.001$ 
Table 34. Spearman correlation coefficients of the EQ-5D summary score, sub-scores and visual analogue scale with the generic (SF 36) and the disease specific (PDQ 39) quality of life measures

\begin{tabular}{|c|c|c|c|c|c|c|c|}
\hline & $\begin{array}{l}\text { EQ-5D } \\
\text { Mobility }\end{array}$ & Selfcare & Usual activities & Pain & Anxiety & Summary index & Visual analogue score \\
\hline \multicolumn{8}{|l|}{ PDQ 39 SI } \\
\hline Mobility & $0.73^{\star \star \star}$ & $0.59^{\star \star \star}$ & $0.67^{\star \star \star}$ & $0.54^{\star \star \star}$ & $0.47^{\star \star \star}$ & $-0.74^{\star \star \star *}$ & $-0.56^{\star \star \star}$ \\
\hline $\mathrm{ADL}$ & $0.65^{\star \star \star}$ & $0.72^{\star \star \star \star}$ & $0.65^{\star \star \star}$ & $0.37^{\star \star \star}$ & $0.38^{\star \star \star}$ & $-0.63^{\star \star \star}$ & $-0.41^{\star \star \star}$ \\
\hline Emotional & $0.54^{\star \star \star}$ & $0.42^{\star \star \star}$ & $0.46^{\star \star \star}$ & $0.37^{\star \star \star}$ & $0.50^{\star \star \star}$ & $-0.54^{\star \star \star}$ & $-0.43^{\star \star \star}$ \\
\hline Stigma & $0.34^{\star \star \star}$ & $0.30^{\star \star}$ & $0.33^{\star \star}$ & $0.35^{\star \star \star}$ & $0.48^{\star * *}$ & $-0.41^{* \star \star}$ & $-0.47^{\star \star \star}$ \\
\hline Social Functioning & $0.26^{*}$ & $0.28^{\star \star}$ & $0.28^{\star \star}$ & $0.31^{\star \star}$ & $0.26^{\star}$ & $-0.35^{\star \star \star}$ & $-0.30^{\star *}$ \\
\hline Cognitions & $0.49^{\star \star \star}$ & $0.51^{\star \star \star}$ & $0.58^{\star \star \star}$ & $0.35^{\star \star \star}$ & $0.45^{\star \star \star}$ & $-0.55^{\star \star \star}$ & $-0.47^{\star \star \star}$ \\
\hline Communication & $0.53^{\star \star \star}$ & $0.58^{\star \star \star}$ & $0.57^{\star \star \star}$ & $0.34^{\star \star}$ & $0.28^{\star \star}$ & $-0.56^{\star \star \star}$ & $-0.42^{\star \star \star}$ \\
\hline Bodily & $0.40^{\star \star \star}$ & $0.35^{\star \star \star}$ & $0.45^{\star \star \star}$ & $0.47^{\star \star \star}$ & $0.40^{\star \star \star}$ & $-0.55^{\star \star \star}$ & $-0.51^{\star \star *}$ \\
\hline Summary score & $0.69^{\star \star \star}$ & $0.63^{\star \star \star}$ & $0.677^{\star \star \star}$ & $0.54^{\star \star \star}$ & $0.55^{\star \star \star}$ & $-0.75^{\star \star \star}$ & $-0.60^{\star \star \star}$ \\
\hline \multicolumn{8}{|l|}{ SF-36 } \\
\hline Physcial functioning & $-0.58^{\star \star \star}$ & $-0.44^{\star \star \star}$ & $-0.52^{\star \star \star}$ & $-0.42^{\star \star \star}$ & $-0.36^{\star \star \star}$ & $0.57^{\star \star \star}$ & $0.47^{\star \star \star}$ \\
\hline Physical role & $-0.42^{\star \star \star}$ & -0.18 & $-0.40^{\star \star \star}$ & -0.40 & $-0.27^{\star}$ & $0.43^{\star \star \star}$ & $0.43^{\star \star \star}$ \\
\hline Bodily pain & $-0.35^{\star \star \star}$ & $-0.33^{\star \star \star}$ & $-0.36^{\star \star \star}$ & $-0.59^{\star \star \star}$ & $-0.32^{\star \star}$ & $0.56^{\star \star \star}$ & $0.54^{\star \star \star}$ \\
\hline General health & $-0.37^{\star \star \star}$ & $-0.40^{\star \star \star}$ & $-0.48^{\star \star \star}$ & $-0.36^{\star \star \star}$ & $-0.35^{\star \star \star}$ & $0.47^{\star \star \star}$ & $0.62^{\star \star \star}$ \\
\hline Vitality & $-0.39^{\star \star \star}$ & $-0.46^{\star \star \star}$ & $-0.48^{\star \star \star}$ & $-0.34^{\star \star}$ & $-0.46^{\star \star \star}$ & $0.52^{\star \star \star}$ & $0.49^{\star \star \star}$ \\
\hline Social Functioning & $-0.56^{\star \star \star}$ & $-0.52^{\star \star \star}$ & $-0.52^{\star \star \star}$ & $-0.46^{\star \star \star}$ & $-0.46^{\star \star \star}$ & $0.59^{\star \star \star}$ & $0.53^{\star * *}$ \\
\hline Emotional role & -0.19 & -0.13 & -0.19 & -0.11 & -0.21 & 0.20 & $0.25^{\star}$ \\
\hline Mental health & $-0.32^{\star \star \star}$ & $-0.43^{\star \star \star}$ & $-0.45^{\star \star \star}$ & $-0.36^{\star \star \star}$ & $-0.53^{\star \star *}$ & $0.50^{\star \star \star}$ & $0.49^{\star \star \star}$ \\
\hline Physical score & $-0.52^{\star \star \star}$ & $-0.41^{\star \star \star}$ & $-0.51^{\star \star \star}$ & $-0.53^{\star \star \star}$ & $-0.31^{\star \star}$ & $0.61^{\star \star \star}$ & $0.55^{\star \star \star}$ \\
\hline Mental score & $-0.25^{\star}$ & $-0.33^{\star \star \star}$ & $-0.34^{\star \star \star}$ & -0.20 & $-0.41^{* * \star}$ & $0.35^{\star \star \star}$ & $0.40^{\star \star \star}$ \\
\hline
\end{tabular}

${ }^{\star} p<0.05,{ }^{\star \star} p<0.01,{ }^{\star \star \star} p<0.001$ 
Table 35. Clinical features associated with significantly impaired QoL scores

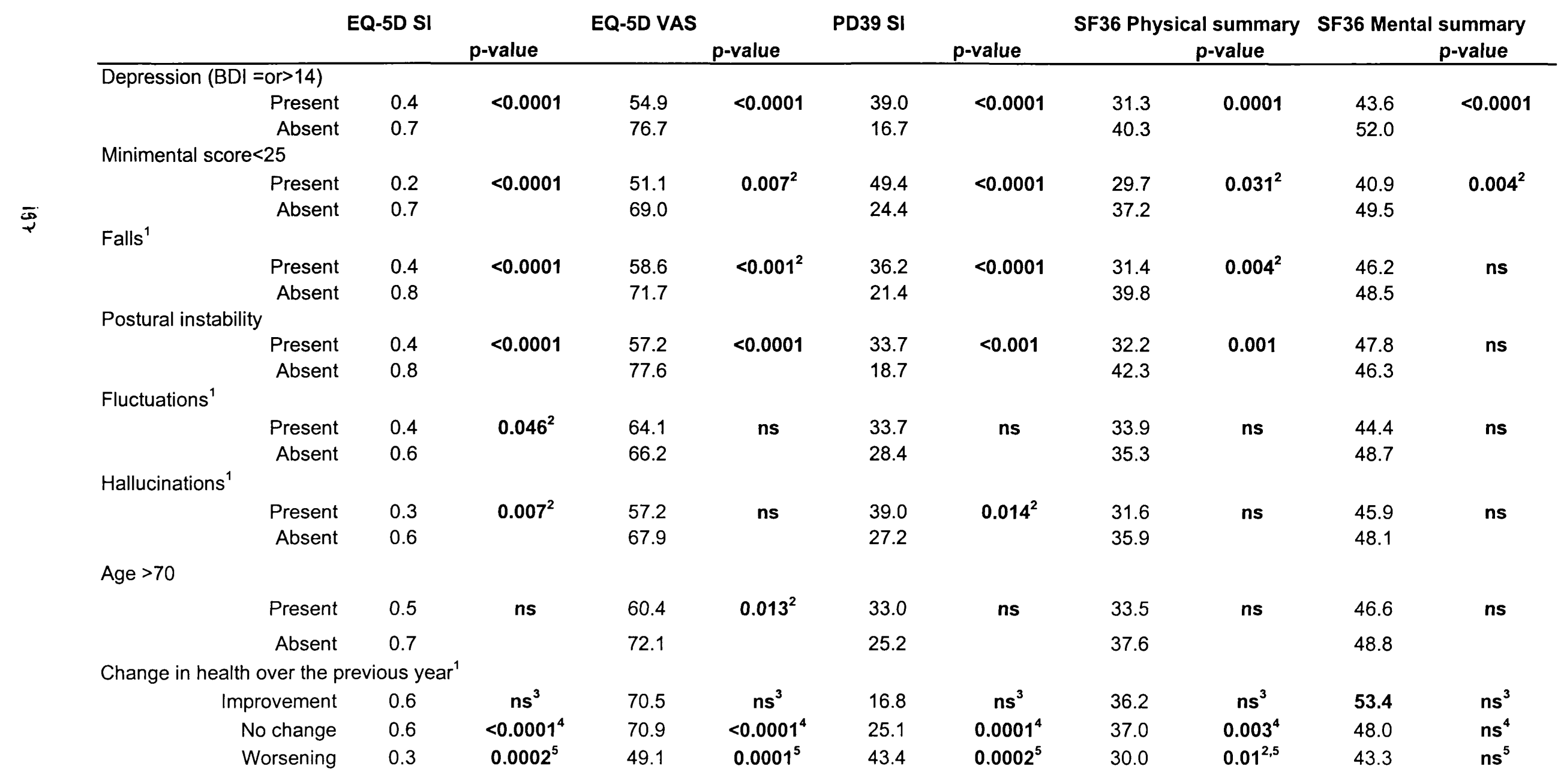

VAS=visual analogue scale; SI-summary index, s. $=$ summary score; ${ }^{1}$ self-reported, ${ }^{2}$ marginally significant, ${ }^{3}$ improvement compared to no change, ${ }^{4}$ no change compared to worsening, ${ }^{5}$ Kruskall Wallis test for all three levels 
Table 36. The percentage of respondents reporting problems on the various dimensions of the EQ-5D by age group

\begin{tabular}{|c|c|c|c|c|c|c|c|}
\hline & & e grou & & & & & \\
\hline & & $30-39$ & $40-49$ & $50-59$ & $60-69$ & $70-79$ & $>80$ \\
\hline General UK population & $\mathrm{n}=$ & 683 & 543 & 461 & 475 & 406 & 140 \\
\hline PD patients & $\mathrm{n}=$ & 2 & 1 & 6 & 23 & 37 & 28 \\
\hline EuroQol dimension & & & & & & & \\
\hline Mobility & & & & & & & \\
\hline General UK population & & 7.8 & 10.3 & 21.9 & 29.3 & 39.8 & 56.7 \\
\hline PD patients & & 50 & 100 & 66.7 & 63.6 & 63.9 & 100 \\
\hline Self-care & & & & & & & \\
\hline General UK population & & 1.6 & 4.2 & 5.2 & 5.7 & 7.4 & 16.3 \\
\hline PD patients & & 50 & 0 & 50 & 45.5 & 47.2 & 42.3 \\
\hline Social functioning & & & & & & & \\
\hline General UK population & & 8.6 & 10.8 & 21.9 & 24.7 & 26.3 & 44 \\
\hline PD patients & & 50 & 0 & 100 & 54.5 & 61.1 & 84.6 \\
\hline Pain & & & & & & & \\
\hline General UK population & & 19.3 & 25.9 & 43.7 & 46.2 & 56 & 60.3 \\
\hline PD patients & & 50 & 0 & 83.3 & 54.5 & 63.9 & 69.2 \\
\hline Anxiety/depression & & & & & & & \\
\hline General UK population & & 17.4 & 18.7 & 27.2 & 28 & 25.3 & 24.8 \\
\hline PD patients & & 50 & 100 & 33.3 & 40.9 & 47.2 & 73.1 \\
\hline
\end{tabular}

PD=Parkinson's disease 
Table 37. Mean scores and standard deviations (in parentheses) of the three quality of life measures as a function of Hoehn and Yahr stage of illness

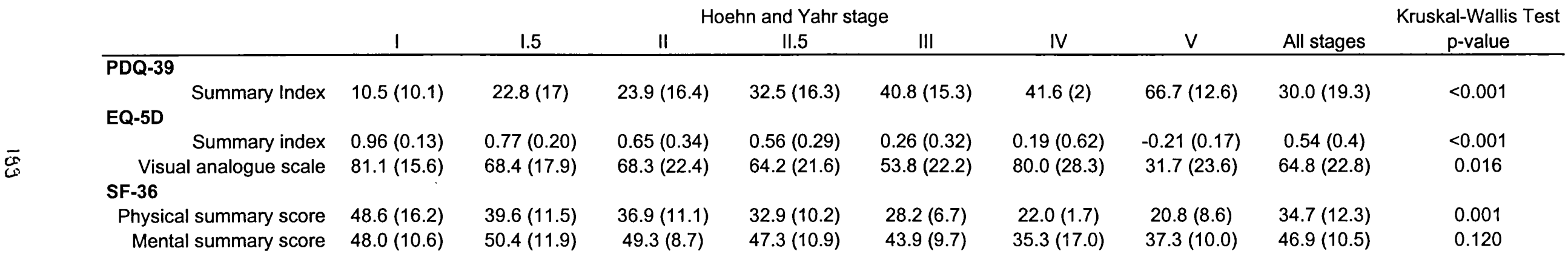


Table 38. Mean (SD) SF 36 scores, by age in comparison to the general UK population*

Age group (years)

$$
35-44
$$

$45-54$

55-64

65-74

\begin{tabular}{|c|c|c|c|c|c|c|c|c|}
\hline & UK population & PD patients & UK population & PD patients & UK population & PD patients & UK population & PD patients \\
\hline Physical functioning & 89 & $62.5(53.0)$ & 84 & $68.7(10.3)$ & 74 & $62.9(24.0)$ & 60 & $50.8(32.3)$ \\
\hline Social functioning & 87 & $75.0(35.4)$ & 87 & $87.5(17.7)$ & 84 & $65.0(25.6)$ & 80 & $59.9(27.4)$ \\
\hline \multicolumn{9}{|l|}{ Role limitations: } \\
\hline physical & 81 & $50.0(70.7)$ & 83 & $62.5(32.3)$ & 72 & $62.9(24.0)$ & 59 & $35.3(54.7)$ \\
\hline emotional & 81 & $50.0(70.7)$ & 82 & $83.3(19.2)$ & 80 & $79.0(52.5)$ & 73 & $63.5(52.8)$ \\
\hline Mental health & 70 & $70.0(31.1)$ & 72 & $81.0(17.1)$ & 74 & $62.0(23.1)$ & 73 & $62.6(20.4)$ \\
\hline Vitality & 58 & $50.0(35.4)$ & 59 & $55.0(26.8)$ & 59 & $50.0(18.7)$ & 57 & $41.3(22.1)$ \\
\hline Pain & 87 & $55.0(63.6)$ & 77 & $81(21.9)$ & 73 & $61.4(32.8)$ & 67 & $62.2(28.7)$ \\
\hline General health perceptions & 72 & $43.5(33.2)$ & 70 & $49.0(24.0)$ & 65 & $48.5(19.5)$ & 58 & $45.8(19.9)$ \\
\hline
\end{tabular}

*from Brazier et al (Brazier) 
Table 39. Mean (SD) quality of life scores in patients with Parkinson's disease in this study and previous studies

\begin{tabular}{rlccc} 
& This study & \multicolumn{2}{c}{ Fitzpatrick et al (1) } & Rubenstein et al (2) \\
& & Clinic attenders & Nonclinic & \\
\hline SF-36 & & & & \\
Physical functioning & $46.9(31.8)$ & $49.3(30.8)$ & $29.0(27.6)$ & $55.4(2.1)$ \\
Physical role limitations & $38.1(53.1)$ & $32.4(39.9)$ & $17.9(31.0)$ & $49.7(3.1)$ \\
eneral health perceptions & $61.3(28.2)$ & $63.1(29.4)$ & $54.0(28.5)$ & $64.6(2.0)$ \\
Vitality & $45.2(20.3)$ & $51.9(21.9)$ & $42.5(21.3)$ & $52.9(1.5)$ \\
Social functioning & $60.6(21.3)$ & $43.0(22.5)$ & $35.1(20.4)$ & $48.2(1.6)$ \\
Emotional role limitations & $69.7(53.9)$ & $63.1(31.0)$ & $48.3(32.2)$ & $73.0(1.9)$ \\
Mental health & $65.4(19.8)$ & $65.3(20.9)$ & $60.6(20.4)$ & $73.1(1.2)$ \\
& & & & \\
PDQ-39 & & & & \\
Mobility & $47.0(33.4)$ & $41.7(31.6)$ & $64.2(30.3)$ & \\
Activities of daily living & $36.5(29.7)$ & $40.4(28.0)$ & $56.3(28.0)$ & \\
Emotional well-being & $29.0(22.5)$ & $32.2(22.3)$ & $40.4(23.2)$ & \\
Stigma & $23.6(27.7)$ & $30.8(26.2)$ & $35.1(28.5)$ & \\
Social support & $14.8(20.7)$ & $13.8(20.0)$ & $20.9(22.3)$ & \\
Cognitions & $31.1(22.0)$ & $33.7(23.1)$ & $48.0(23.3)$ & \\
Communication & $20.3(22.6)$ & $25.9(23.0)$ & $36.1(26.3)$ & \\
Bodily discomfort & $37.4(22.7)$ & $41.0(28.1)$ & $52.7(25.7)$ &
\end{tabular}

EuroQuol

$\begin{array}{rc}\text { Mobility } & 1.8(0.6) \\ \text { Self-care } & 1.5(0.6) \\ \text { Pain } & 1.8(0.7) \\ \text { Social Functioning } & 1.9(0.7) \\ \text { Anxiety/depression } & 1.6(0.6) \\ \text { Summary index } & 0.5(0.4) \\ \text { Visual analogue scale } & 64.0(22.8)\end{array}$


Table 40. Results of stepwise multiple regression analysis of PDQ 39 scores

\begin{tabular}{|c|c|c|c|c|c|}
\hline Predictors & $\begin{array}{c}\text { Standardized Regression } \\
\text { Coefficient }\end{array}$ & $\mathrm{R}$ square & $\begin{array}{c}\text { R square } \\
\text { change }\end{array}$ & t value & $\begin{array}{c}\text { Significance } \\
(p<)\end{array}$ \\
\hline Beck Depression Inventory score & 0.47 & & 0.54 & 5.45 & 0.0001 \\
\hline Schwab and England disability score & -0.31 & 0.64 & 0.1 & 3.41 & 0.001 \\
\hline Minimental state examination score & -0.17 & 0.72 & 0.02 & 2.14 & 0.037 \\
\hline
\end{tabular}

Multiple $\mathrm{R}=0.85$

$\mathrm{R} 2=0.72$

Adjusted $\mathrm{R}^{2}=0.70$ 
Fig. 1 Age-specific prevalence rates of Parkinson's disease per 100,000 (95\% confidence intervals) for all United Kingdom studies of Parkinson's disease

Age groups

Brewis et al 1961

Sutcliffe 1982

Mutch et al 1984

Sutcliffe et al 1992

Schrag et al 1997

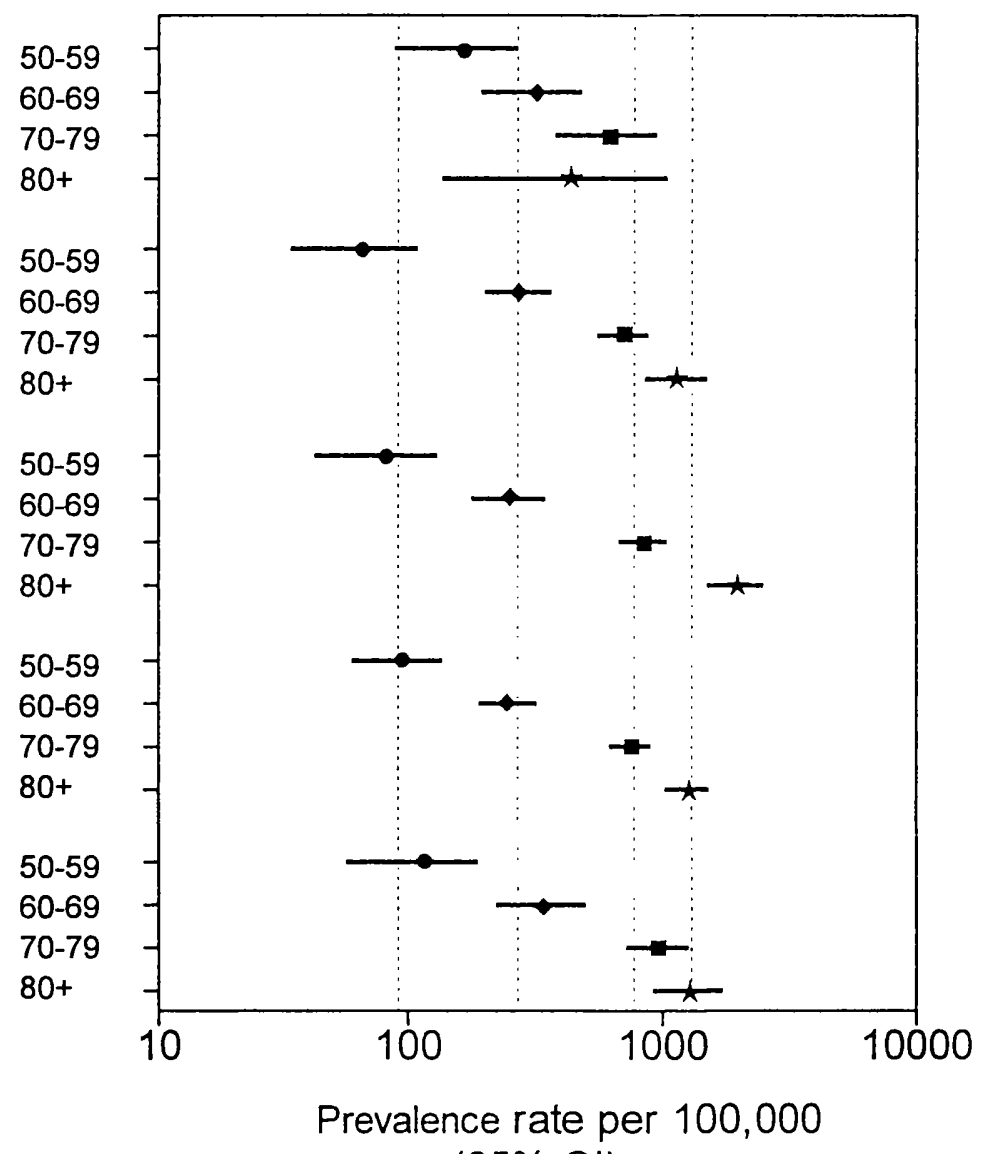

$(95 \% \mathrm{Cl})$ 
Fig. 2. Age-specific prevalence rates of Parkinson's disease in this study and previous studies

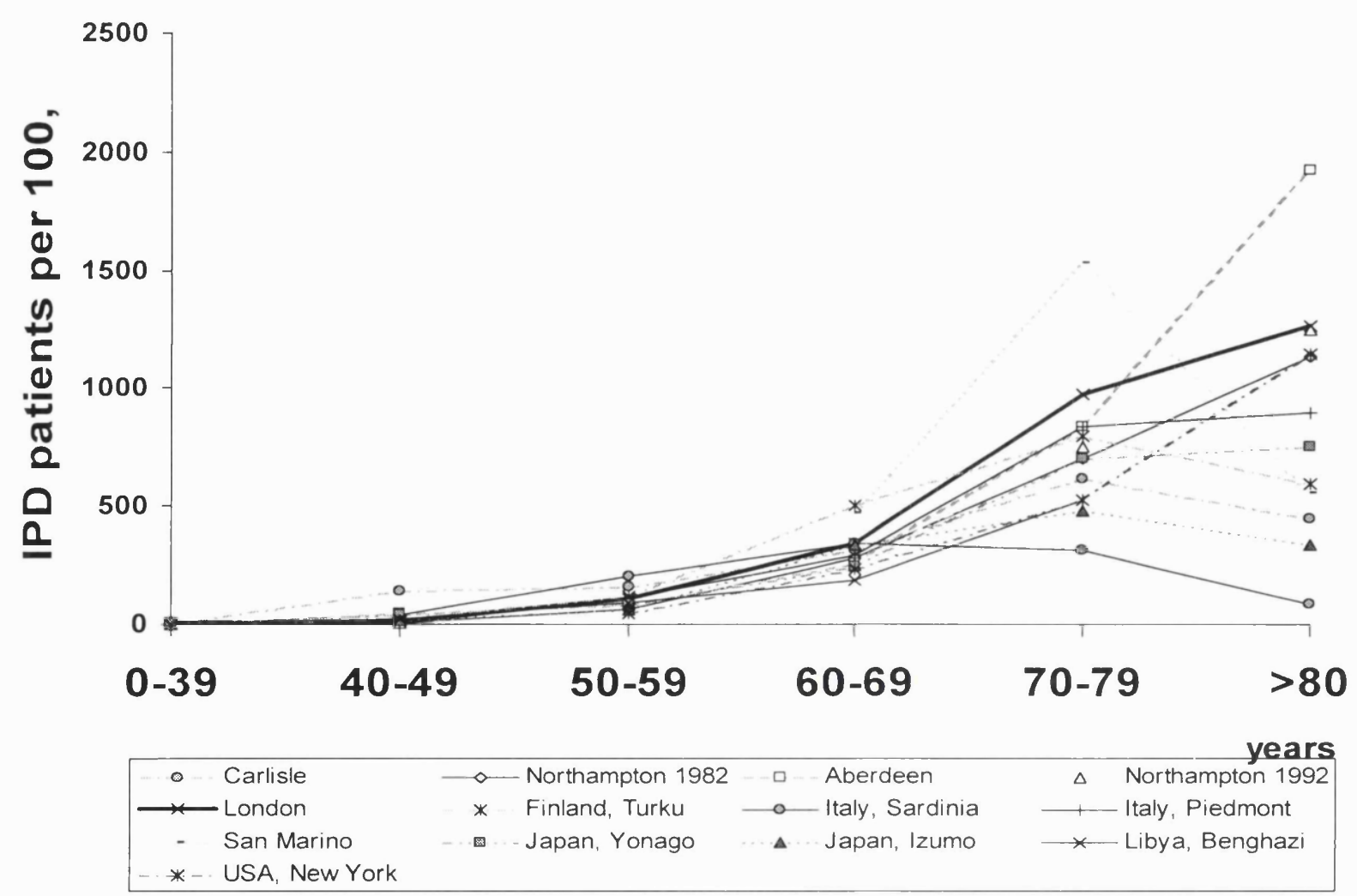

*studies that provided information on age-specific rates, excluding door-to-door studies 
Fig. 3. Percent of patients in each Hoehn and Yahr stage

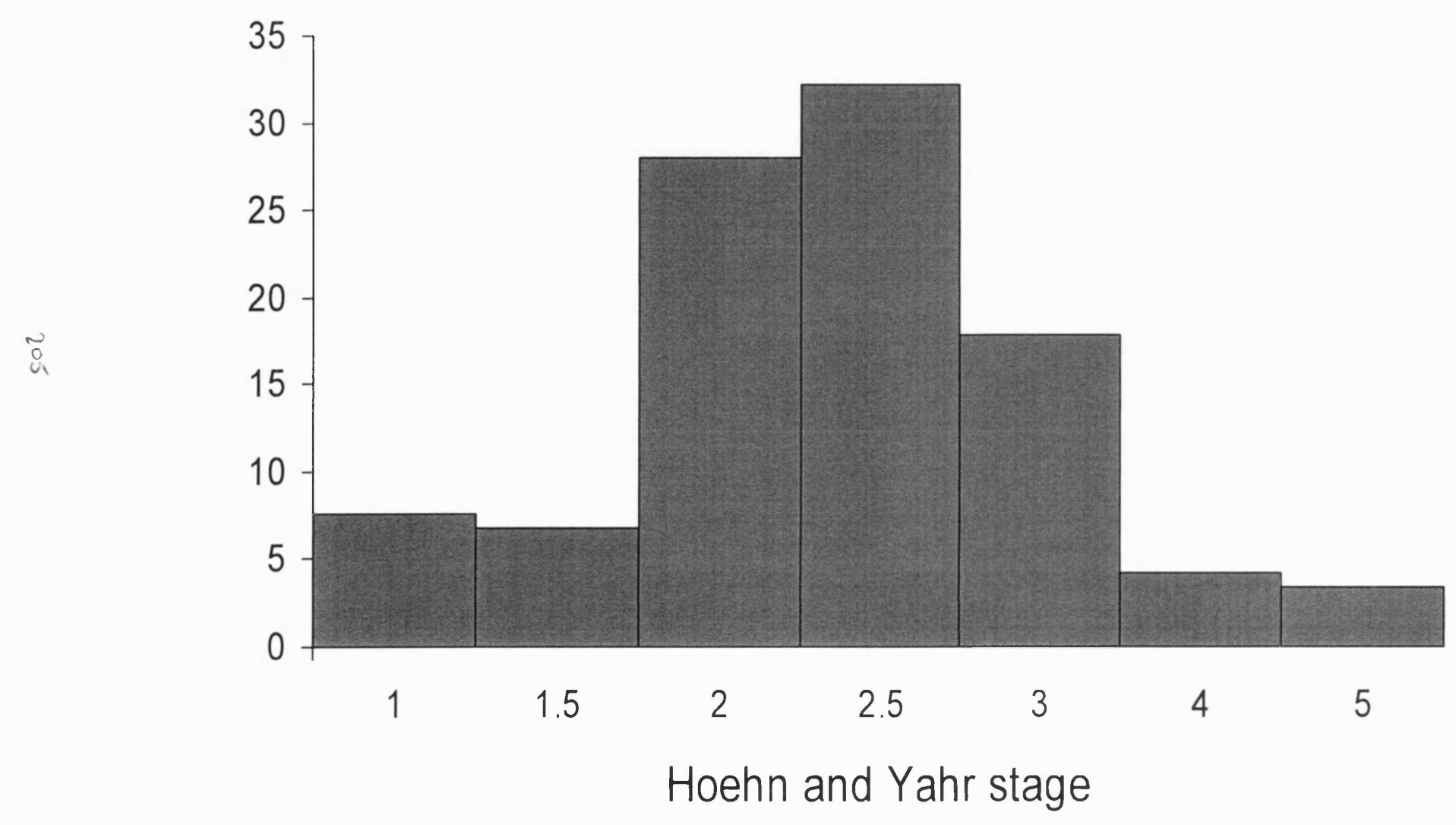




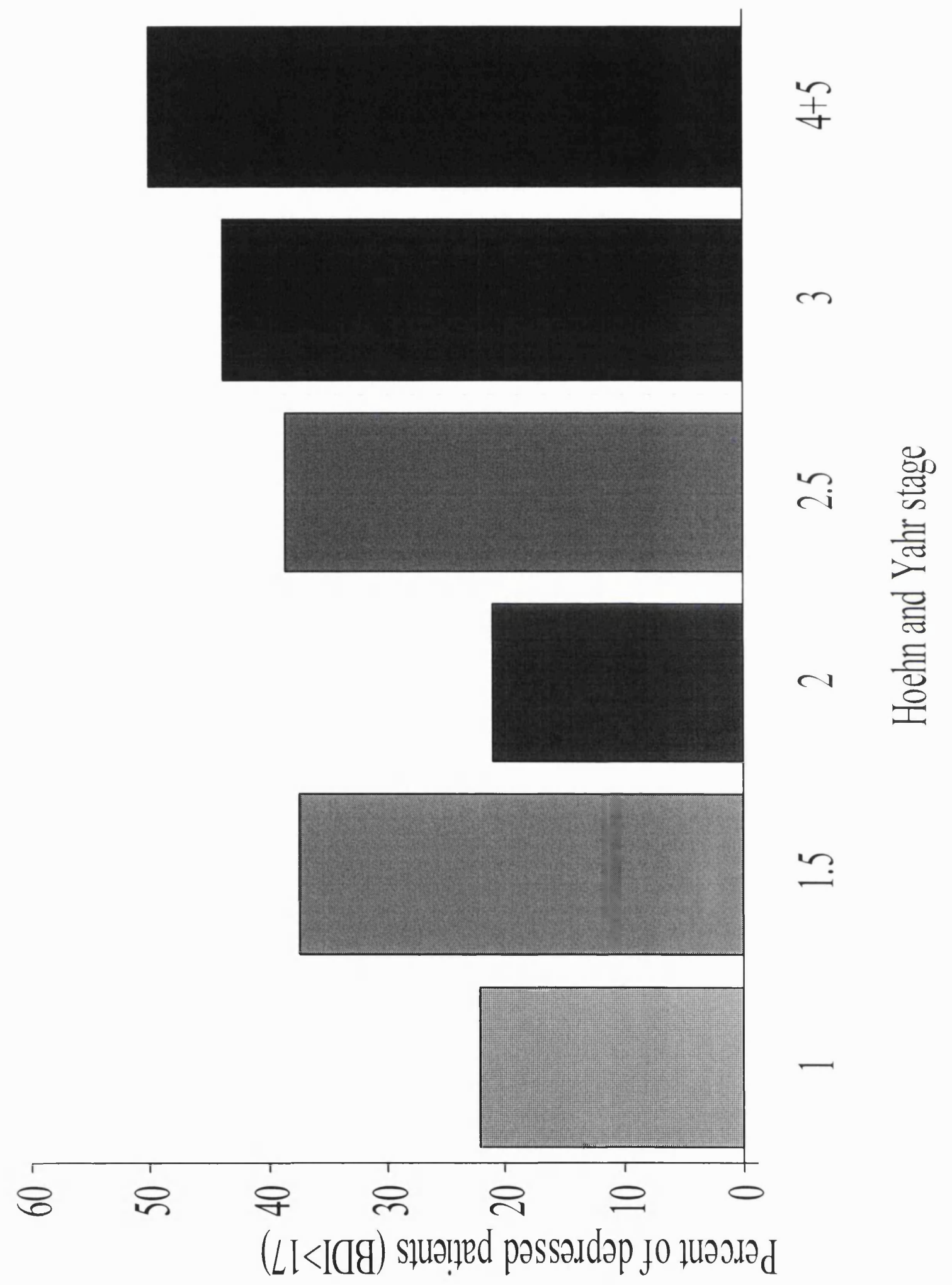


Fig. 5. Rate of prediction of depression scores by parameters of impairment, disability and handicap

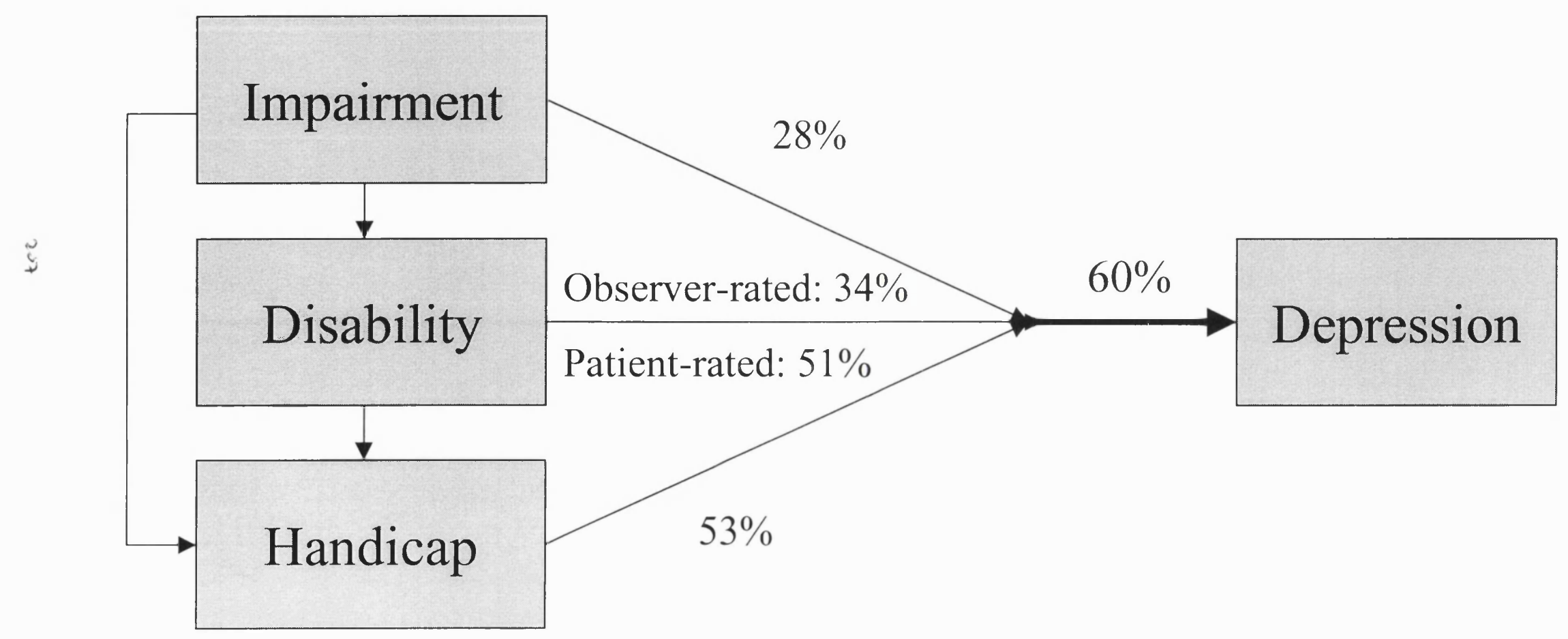


Fig. 6. Number of patients with onset of Parkinson's disease before age 40, by age at onset

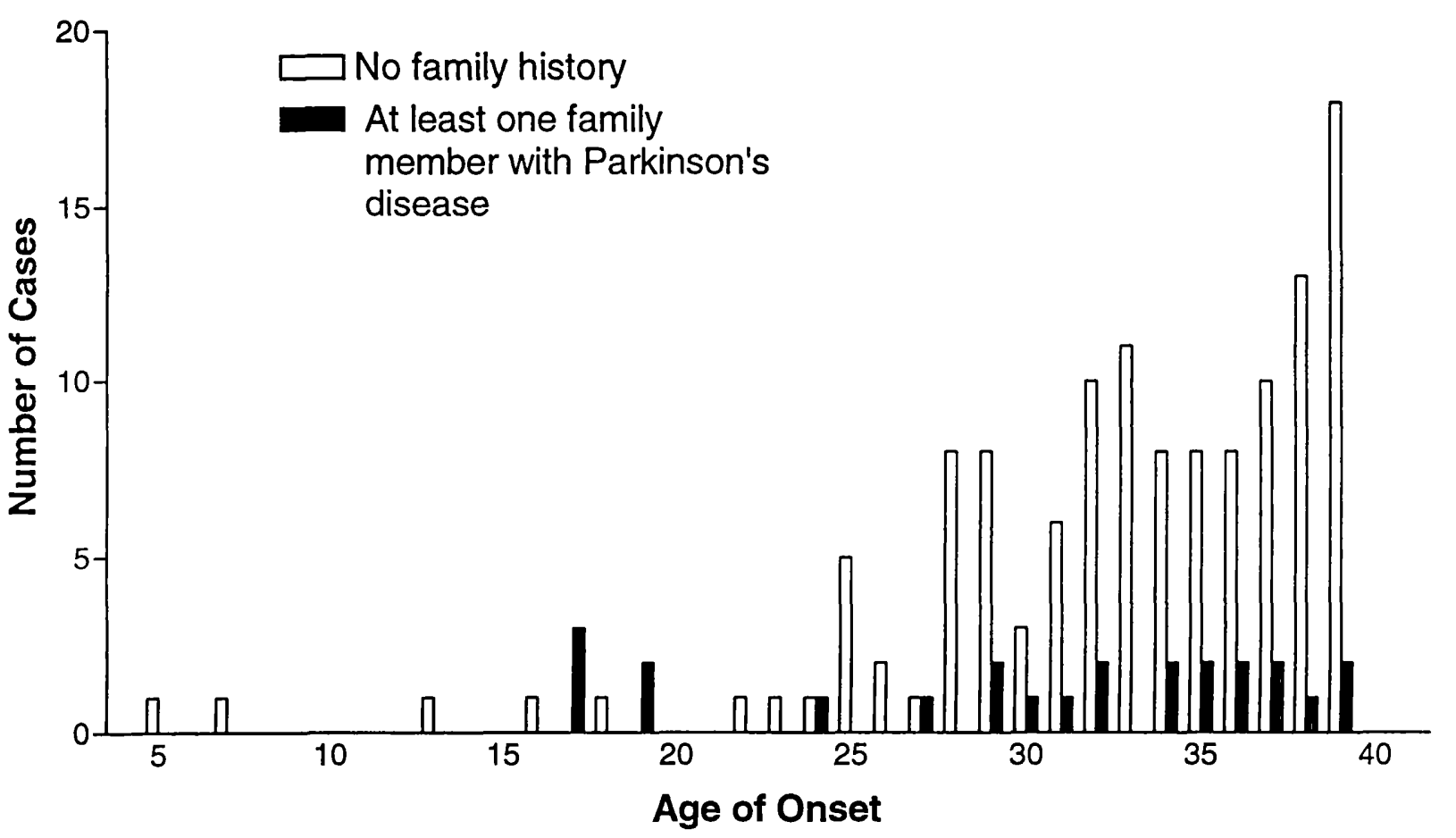


Fig. 7. Cumulative percentage of patients with onset of Parkinson's disease before age 40 and dyskinesias and motor fluctuations, by treatment duration

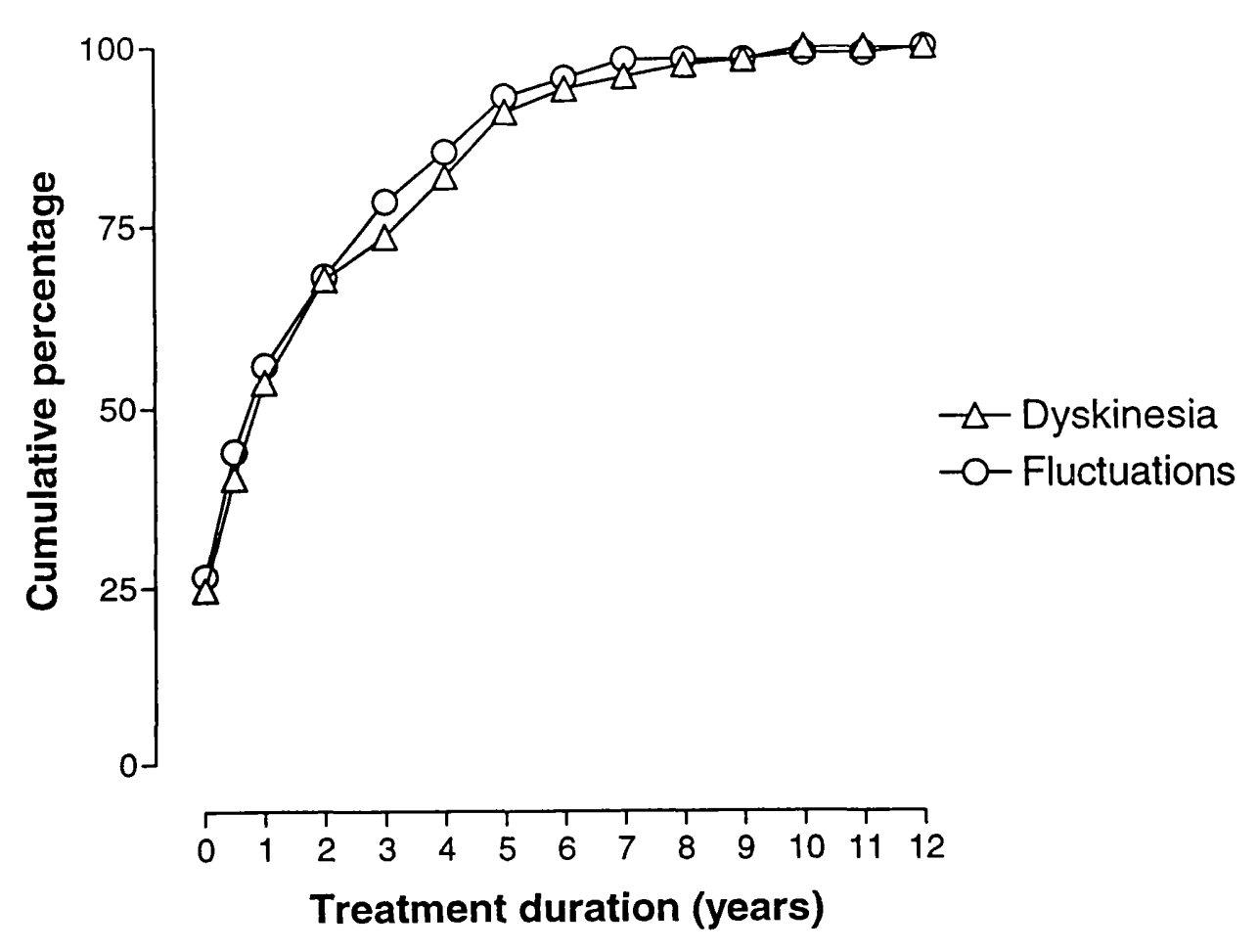


Fig. 8. Percent of patients indicating a problem on the EQ-5D

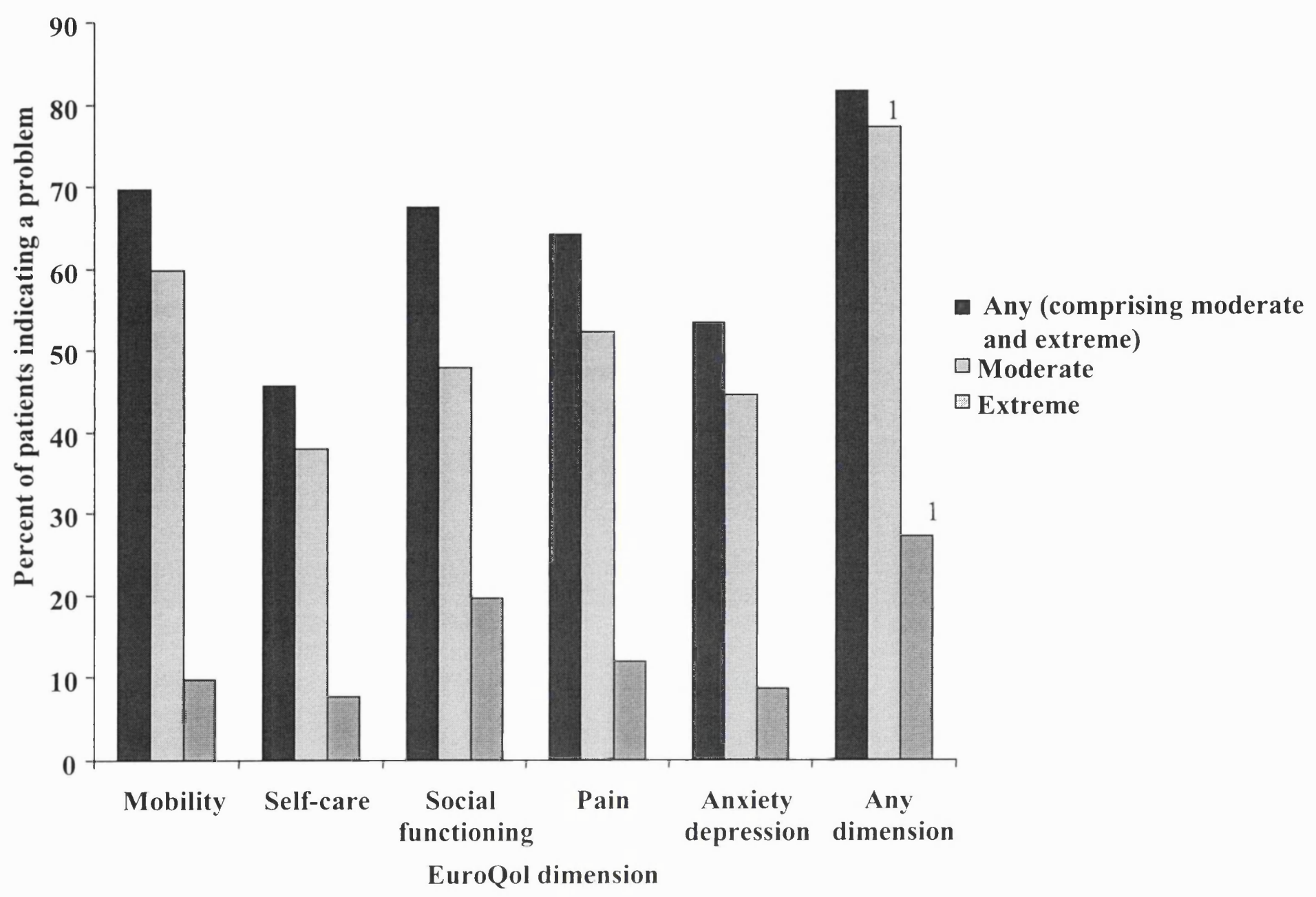

${ }^{\mathrm{l}}$ moderate and extreme problems are not mutually exclusive 
Fig. 9. Mean VAS scores of patients with PD compared to norms from the general population

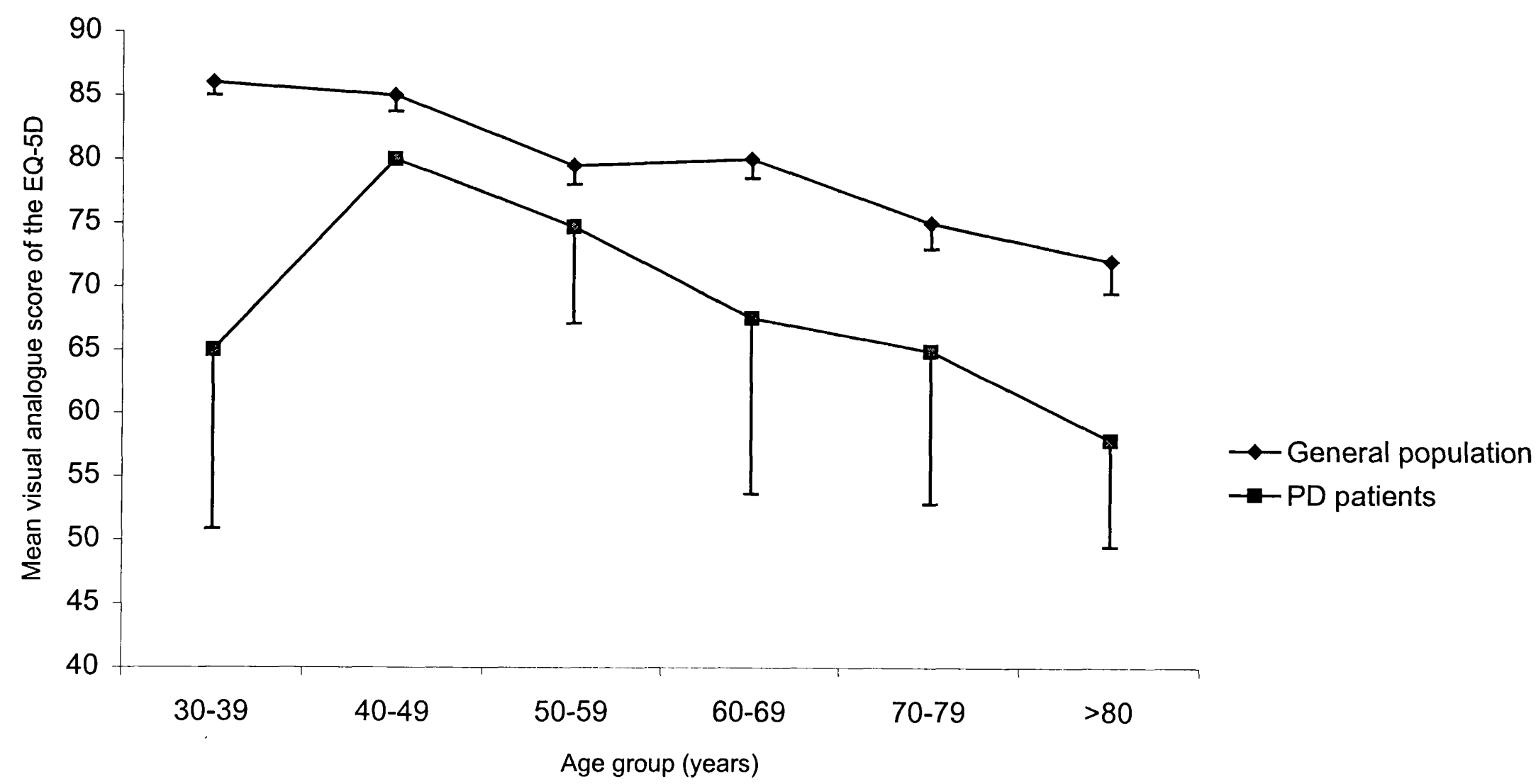

Error bars indicate standard deviations of the means 
Fig. 10. Percent of patients indicating a problem on the EQ-5D by Hoehn and Yahr stage

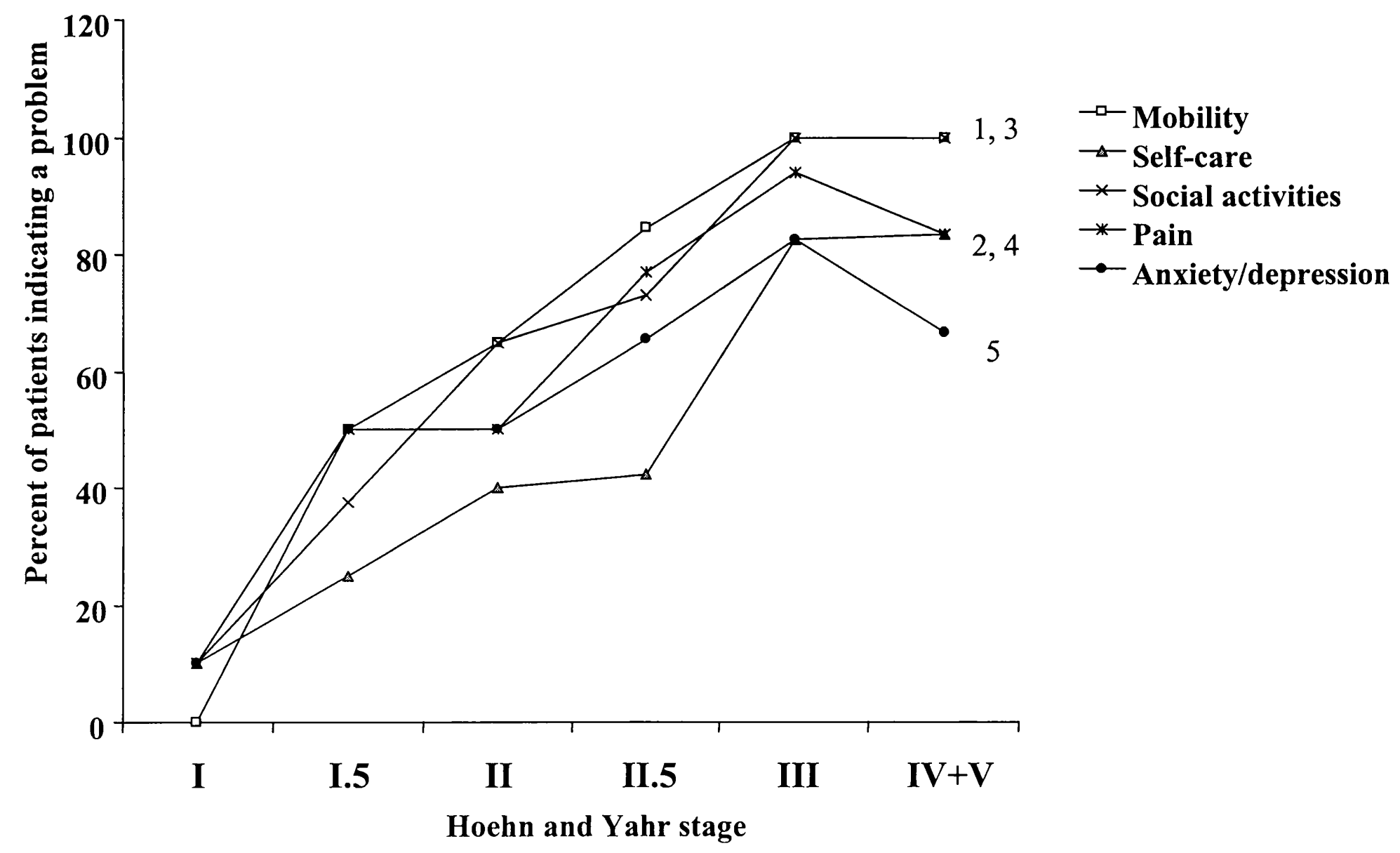

${ }^{1} \mathrm{c}=0.59,{ }^{2} \mathrm{p}=0.54,{ }^{3} \mathrm{c}=0.58,{ }^{4} \mathrm{c}=0.54,{ }^{5} \mathrm{c}=0.41$, all $\mathrm{p}<0.0001$ 
Fig. 11. Mean SF 36 scores, by disease severity

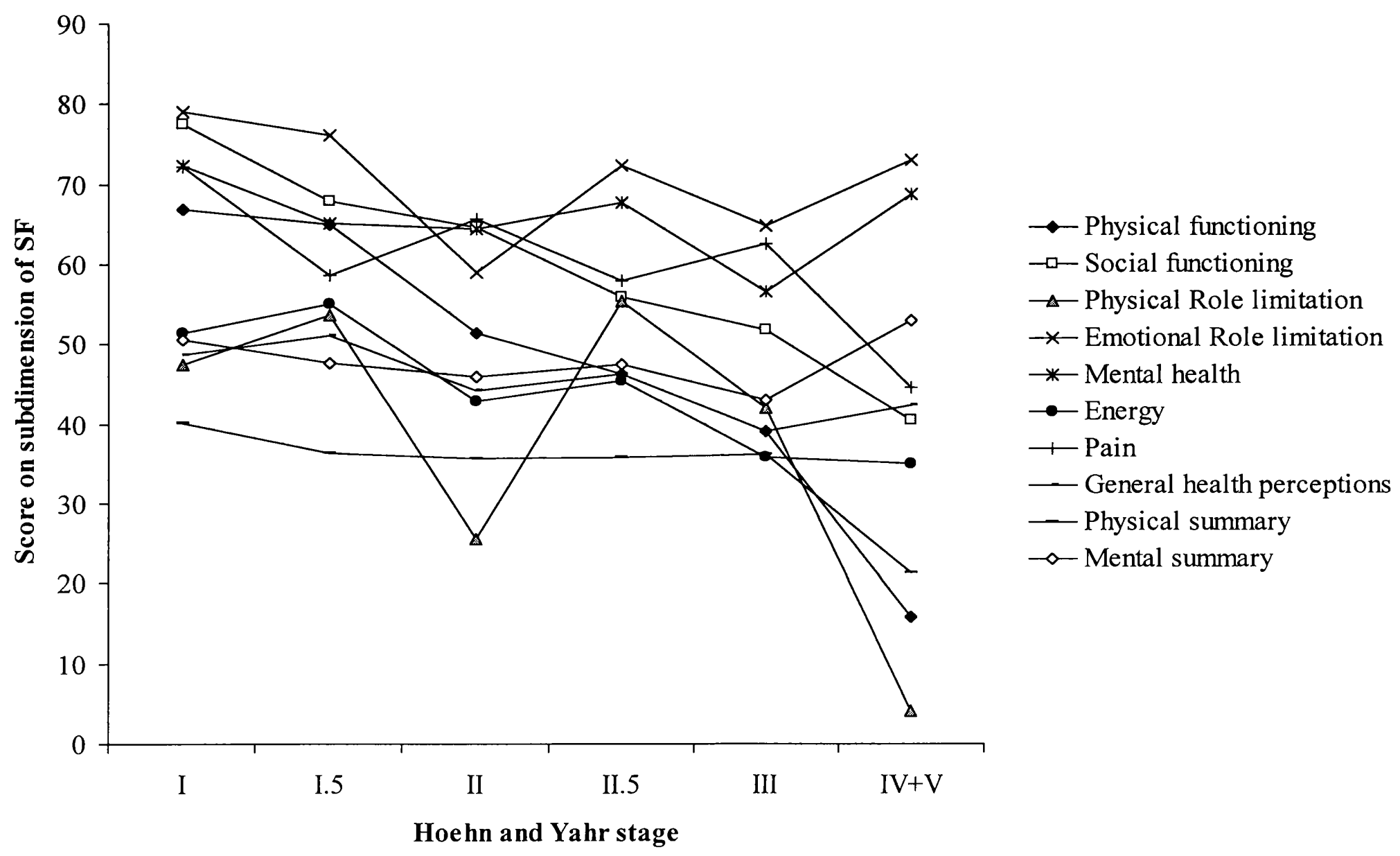


Fig. 12. Mean PDQ 39, by disease severity

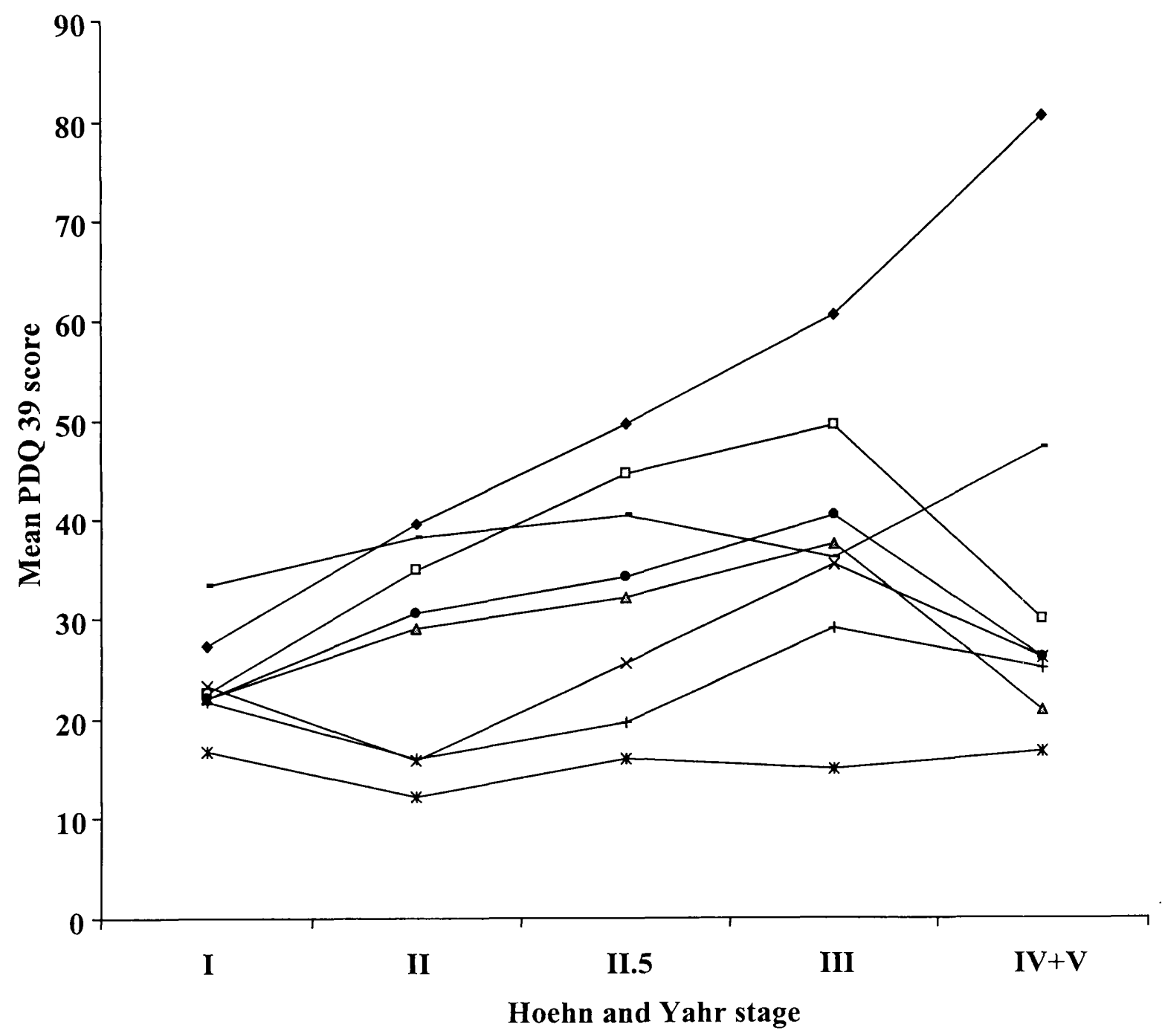

$\rightarrow-$ Mobility

$\rightarrow-$ Activities of daily living

$\triangle$ Emotional well-being

* Stigma

$\rightarrow$-Social support

$\rightarrow-$ Cognitions

+ Communication

- Bodily discomfort 
Appendix 1. Definition of terms used in the analysis of accuracy of diagnosis

Final diagnosis

Parkinson's disease Not Parkinson's disease

Previous diagnosis

Parkinson's disease

A $\quad$ B

Not Parkinson's disease $\quad \mathrm{C} \quad \mathrm{D}$

Sensitivity: Proportion of patients with a final diagnosis of Parkinson's disease (PD) who were previously diagnosed of $\mathrm{PD}(\mathrm{A} / \mathrm{A}+\mathrm{C})$.

Specificity: Proportion of patients without a final diagnosis of PD who were previously diagnosed of not having $\mathrm{PD}(\mathrm{D} / \mathrm{D}+\mathrm{B})$.

Positive predictive value: Proportion of patients with a previous diagnosis of $\mathrm{PD}$ who received a final diagnosis of $\mathrm{PD}(\mathrm{A} / \mathrm{A}+\mathrm{B})$.

Negative predictive value: Proportion of patients with a previous diagnosis of not having $\mathrm{PD}$ who received a final diagnosis of not having $\mathrm{PD}(\mathrm{D} / \mathrm{D}+\mathrm{C})$. 
Appendix 2. Quality of life instruments

2.1. EQ-5D instrument

\section{$E Q-5 D$}

Health Questionnaire

(English version) 
By placing a tick in one box in each group below, please indicate which statements best describe your own health state today.

\section{Mobility}

I have no problems in walking about

I have some problems in walking about

I am confined to bed

\section{Self-Care}

I have no problems with self-care

I have some problems washing or dressing myself

I am unable to wash or dress myself

Usual Activities (e.g. work, study, housework, family or leisure activities)

I have no problems with performing my usual activities

I have some problems with performing my usual activities

I am unable to perform my usual activities

\section{Pain/Discomfort}

I have no pain or discomfort

I have moderate pain or discomfort

I have extreme pain or discomfort

\section{Anxiety/Depression}

I am not anxious or depressed

I am moderately anxious or depressed

I am extremely anxious or depressed

Compared with my general

level of health over the past 12 months,

my health state today is:

Better

TICK

Much the same

ONE

Worse

BOX 
To help people say how good or bad a health state is, we have drawn a scale (rather like a thermometer) on which the best state you can imagine is marked 100 and the worst state you can imagine is marked 0 .

We would like you to indicate on this scale how good or bad your own health is today, in your opinion. Please do this by drawing a line from the box below to whichever point on the scale indicates how good or bad your health state is today.

\section{Your own} health state today 


\section{Short Form Health Survey (SF-36)}

This survey asks for your views about your health and your ability to perform your usual activities. Please tick the box next to the answer which best describes your views about your health.

1) In general, you would say your health is:

1 Excellent

2 Very good

3 Good

4 Fair

5 Poor

2) Compared to one year ago, how would you rate your health in general now?

1 Much better now than one year ago

2 Somewhat better than one year ago

3 About the same as one year ago

4 Somewhat worse than one year ago

5 Much worse now than one year ago

3) During the past 4 weeks, to what extent has your physical health or emotional problems interfered with your normal social activities with family, friends, neighbours, or groups?

1 Not at all

2 Slightly

3 Moderately

4 Quite a bit

5 Extremely 
4) During the past 4 weeks, how much bodily pain have you had?

1 None

2 Very mild

3 Mild

4 Moderate

5 Severe

6 Very severe

5) During the past 4 weeks, how much did pain interfere with your normal work (outside the home and housework)?

1 Not at all

2 A little bit

3 Moderately

4 Quite a bit

5 Extremely

6) During the past 4 weeks, how much of the time has your physical health or emotional problems interfered with you social activities?

1 All the time

2 Most of the time

3 Some of the time

4 A little of the time

5 None of the time

7) During the past 4 weeks, have you had any of the following problems with your work or other regular daily activities as a result of you physical health? (Tick the box or boxes which apply to you)

Cut down on the amount of time you spent on work or other activities Accomplished less than you would like

Were limited in the kind of work or other activities

Had difficulty performing the work or other activities (i.e. it took extra effort) 
8) During the past 4 weeks, have you had any of the following problems with your work or other regular daily activities as a result of any emotional problems (such as feeling depressed or anxious)? (Tick the box or boxes which apply to you)

Cut down on the amount of time you spent on work or other activities Accomplished less than you would like

Did not do work or other activities as carefully as usual

9) The following questions are about activities you might do during a typical day. Does your present health limit you in these activities? Please indicate how much by circling the appropriate number.

\begin{tabular}{|l|c|c|c|}
\hline \multicolumn{1}{|c|}{ ACTIVITIES } & $\begin{array}{c}\text { Yes, } \\
\text { Limited } \\
\text { A lot }\end{array}$ & $\begin{array}{c}\text { Yes, } \\
\text { Limited A little }\end{array}$ & $\begin{array}{c}\text { No, Not } \\
\text { Limited At all }\end{array}$ \\
\hline $\begin{array}{l}\text { a. Vigorous activities, such } \\
\text { as running, lifting heavy } \\
\text { objects, or strenuous sports }\end{array}$ & 1 & 2 & 3 \\
\hline $\begin{array}{l}\text { b. Moderate activities, such as } \\
\text { moving a table, pushing a } \\
\text { vacuum cleaner, bowling, or } \\
\text { playing golf }\end{array}$ & 1 & 2 & 3 \\
\hline c. Lifting or carrying groceries & 1 & 2 & 3 \\
\hline $\begin{array}{l}\text { d. Climbing several flights of } \\
\text { stairs }\end{array}$ & 1 & 2 & 3 \\
\hline e. Climbing one flight of stairs & 1 & 2 & 3 \\
\hline $\begin{array}{l}\text { f. Bending, kneeling, or } \\
\text { stooping }\end{array}$ & 1 & 2 & 3 \\
\hline g. Walking more than a mile & 1 & 2 & 3 \\
\hline h. Walking half a mile & 1 & 2 & 3 \\
\hline i. Walking one hundred yards & 1 & 2 & 3 \\
\hline j. Bathing or dressing yourself & 1 & 2 & 3 \\
\hline
\end{tabular}


10) These questions are about how you feel and how things have been with you during the past 4 weeks. For each question, please give the one answer that comes closest to the way you have been feeling. How much of the time during the past 4 weeks... (circle one number for each question)

\begin{tabular}{|l|c|c|c|c|c|c|}
\hline & $\begin{array}{c}\text { All the } \\
\text { time }\end{array}$ & $\begin{array}{c}\text { Most } \\
\text { of the } \\
\text { time }\end{array}$ & $\begin{array}{c}\text { A Good } \\
\text { Bit of } \\
\text { the time }\end{array}$ & $\begin{array}{c}\text { Some } \\
\text { of the } \\
\text { time }\end{array}$ & $\begin{array}{c}\text { A little } \\
\text { of the } \\
\text { time }\end{array}$ & $\begin{array}{c}\text { None } \\
\text { of the } \\
\text { time }\end{array}$ \\
\hline Did you feel full of life? & 1 & 2 & 3 & 4 & 5 & 6 \\
\hline $\begin{array}{l}\text { Have you been a very nervous } \\
\text { person? }\end{array}$ & 1 & 2 & 3 & 4 & 5 & 6 \\
\hline $\begin{array}{l}\text { Have you felt so down in the } \\
\text { dumps that nothing could cheer } \\
\text { you up? }\end{array}$ & 1 & 2 & 3 & 4 & 5 & 6 \\
\hline Have you felt calm and peaceful? & 1 & 2 & 3 & 4 & 5 & 6 \\
\hline Did you have a lot of energy? & 1 & 2 & 3 & 4 & 5 & 6 \\
\hline $\begin{array}{l}\text { Have you felt downhearted and } \\
\text { low? }\end{array}$ & 1 & 2 & 3 & 4 & 5 & 6 \\
\hline Did you feel worn out? & 1 & 2 & 3 & 4 & 5 & 6 \\
\hline Have you been a happy person? & 1 & 2 & 3 & 4 & 5 & 6 \\
\hline Did you feel tired? & 1 & 2 & 3 & 4 & 5 & 6 \\
\hline
\end{tabular}

11) How true or false is each of the following statements for you? (Please circle one number for each line)

\begin{tabular}{|l|c|c|c|c|c|}
\hline & $\begin{array}{c}\text { Definitely } \\
\text { True }\end{array}$ & $\begin{array}{c}\text { Mostly } \\
\text { true }\end{array}$ & $\begin{array}{c}\text { Don't } \\
\text { know }\end{array}$ & $\begin{array}{c}\text { Mostly } \\
\text { false }\end{array}$ & $\begin{array}{c}\text { Definitely } \\
\text { false }\end{array}$ \\
\hline $\begin{array}{l}\text { I seem to get ill a little } \\
\text { easier than other people }\end{array}$ & 1 & 2 & 3 & 4 & 5 \\
\hline $\begin{array}{l}\text { I am as healthy as } \\
\text { anybody I know }\end{array}$ & 1 & 2 & 3 & 4 & 5 \\
\hline $\begin{array}{l}\text { I expect my health to get } \\
\text { worse over time }\end{array}$ & 1 & 2 & 3 & 4 & 5 \\
\hline My health is excellent & 1 & 2 & 3 & 4 & 5 \\
\hline
\end{tabular}


2.3. The PDQ 39 instrument

\section{The Parkinson's disease questionnaire-PDQ39}

Due to having illness, how often have you experienced the following, during the last month? Please tick one box for each question

\begin{tabular}{|c|c|c|c|c|c|}
\hline & Never & Occasionally & Sometimes & Often & $\begin{array}{c}\text { Always or } \\
\text { cannot do } \\
\text { at all }\end{array}$ \\
\hline \multicolumn{6}{|c|}{$\begin{array}{l}\text { 1. Had difficulty doing the leisure activities you } \\
\text { would like to do? }\end{array}$} \\
\hline \multicolumn{6}{|l|}{$\begin{array}{l}\text { 2. Had difficulty looking after your home, e.g. } \\
\text { DIY, housework, cooking? }\end{array}$} \\
\hline \multicolumn{6}{|l|}{ 3. Had difficulty carrying bags of shopping? } \\
\hline \multicolumn{6}{|l|}{ 4. Had problem walking half a mile? } \\
\hline \multicolumn{6}{|l|}{ 5. Had problems walking 100 yards? } \\
\hline \multicolumn{6}{|l|}{$\begin{array}{l}\text { 6. Had problem getting around the house as } \\
\text { easily as you would like? }\end{array}$} \\
\hline \multicolumn{6}{|l|}{ 7. Had difficulty getting around in public? } \\
\hline \multicolumn{6}{|l|}{$\begin{array}{l}\text { 8. Needed someone else to accompany you } \\
\text { when you went out? }\end{array}$} \\
\hline \multicolumn{6}{|l|}{$\begin{array}{l}\text { 9. Felt frightened or worried about falling over } \\
\text { in public? }\end{array}$} \\
\hline \multicolumn{6}{|c|}{$\begin{array}{l}\text { 10. Have been confined to the house more than } \\
\text { you would like? }\end{array}$} \\
\hline \multicolumn{6}{|l|}{ 11. Had difficulty washing yourself? } \\
\hline \multicolumn{6}{|l|}{ 12. Had difficulty dressing yourself? } \\
\hline \multicolumn{6}{|l|}{$\begin{array}{l}\text { 13. Had problems doing up buttons and } \\
\text { shoelaces? }\end{array}$} \\
\hline \multicolumn{6}{|l|}{ 14. Had problems writing clearly? } \\
\hline \multicolumn{6}{|l|}{ 15. Had difficulty cutting up your food? } \\
\hline $\begin{array}{l}\text { 16. Had difficulty holding a drink without } \\
\text { spilling it? }\end{array}$ & & & & & \\
\hline
\end{tabular}

\section{Continued on next page.}




\section{Appendix 3. The Beck Depression Inventory}

\section{Beck Depression Inventory}

On this questionnaire are groups of statements. Please read each group of statements carefully. Then pick out the one statement in each group, which best describes the way you have been feeling during the past week, including today. Circle the number next to the statement you have chosen. If several statements in the group seem to apply equally well, then circle each number which applies to you.

Be sure to read all the statements in each group before making your choice.

1)

0 I do not feel sad.

1 I feel sad.

2 I am sad all the time and I can't snap out of it.

3 I am so sad or unhappy that I can't stand it.

2) 0 I am not particularly discouraged about the future.

1 I feel discouraged about the future.

2 I feel I have nothing to look forward to.

3 I feel that the future is hopeless and that things cannot improve.

3) 0 I do not feel like a failure.

1 I feel I have failed more than the average person.

2 As I look back on my life, all I can see is a lot of failures.

3 I feel I am a complete failure as a person.

4) $\quad 0 \quad$ I get as much satisfaction out of things as I used to.

1 I don't enjoy things the way $\mid$ used to.

2 I don't get satisfaction out of anything anymore.

3 I am dissatisfied or bored with everything.

5) 0 I don't feel particularly guilty.

1 I feel guilty a good part of the time.

2 I feel quite guilty most of the time.

3 I feel guilty all of the time.

6) 0 I don't feel I am being punished.

1 I feel I may be punished.

2 I expect to be punished.

3 I feel I am being punished.

7) $0 \quad 1$ don't feel disappointed in myself.

1 I am disappointed in myself.

2 I am disgusted with myself.

3 I hate myself. 
8) 0 I don't feel I am any worse than anybody else.

1 I am critical of myself for my weaknesses or mistakes.

2 I blame myself all the time for my faults.

3 I blame myself for everything bad that happens.

9) 0 I don't have any thoughts of killing myself.

1 I have thoughts of killing myself, but I would not carry them out.

2 I would like to kill myself.

3 I would kill myself if I had the chance.

10) $0 \quad I$ don't cry any more than usual.

1 I cry more now than I used to.

2 I cry all the time now.

3 I used to be able to cry, but now I can't cry even though I want to.

11) 0 I am no more irritated now than I ever am.

1 I get annoyed or irritated more easily than I used to.

2 I feel irritated all the time now.

3 I don't get irritated at all by things that used to irritated me.

12) 0 I have lost not interest in other people.

1 I am less interested in other people than I used to be.

2 I have lost most of my interest in other people.

3 I have lost all of my interest in other people.

13) 0 I make decisions about as well as I ever could.

1 I put off making decisions more than I used to.

2 I have greater difficulty in making decisions than before.

3 I can't make any decisions at all any more.

14) 0 I don't feel I look worse than I used to.

1 I am worried that I am looking old and unattractive.

2 I feel that there are permanent changes in my appearance and they make me look unattractive.

3 I believe that I look ugly.

15) 0 I can work about as well as before.

1 It takes extra effort to get started at doing something.

2 I have to push myself very hard to do anything.

3 I can't do any work at all.

16) 0 I can sleep as well as usual.

1 I don't sleep as well as I used to.

2 I wake up 1-2 hours earlier than usual and find it hard to get back to sleep.

3 I wake up several hours earlier than I used to and cannot get back to sleep. 
17) 0 I don't get any more tired than usual.

1 I get tired more easily than I used to.

2 I get tired from doing almost anything.

$3 \mathrm{I}$ am too tired to do anything.

18) 0 My appetite is no worse than usual.

1 My appetite is not as good as it used to be.

2 My appetite is much worse now.

3 I have no appetite at all any more.

19) 0 । haven't lost much weight, if any lately.

1 I have lost more than 5 pounds.

2 I have lost more than 10 pounds.

3 I have lost more than 15 pounds.

I am purposely trying to lose weight eating less: (Circle) Yes No

20) 0 I am no more worried about my health than usual.

1 I am worried about my physical problems such as aches and pains, upset stomach or constipation.

2 I am very worried about physical problems and it's hard to think about much else.

$3 \mathrm{I}$ am so worried about my physical problems that I cannot think about anything else

21) 0 I have not noticed any recent change in my interest in sex.

1 I am less interested in sex than I used to be.

2 I am much less interested in sex now.

3 I have lost interest in sex completely. 


\section{Appendix 4. Numbers of patients screened and examined in the overall study}

\begin{tabular}{|c|c|c|c|}
\hline & $\begin{array}{c}\text { Overall } \\
\mathbf{n}\end{array}$ & $\begin{array}{c}\text { Examined } \\
\mathbf{n}\end{array}$ & $\begin{array}{c}\text { Not examined } \\
\mathbf{n}\end{array}$ \\
\hline Screened positive & 679 & & \\
\hline Excluded after review of records & 438 & & \\
\hline Eligible & 241 & 202 & 39 \\
\hline Declined & 33 & & \\
\hline Died & 6 & & \\
\hline Seen & 202 & & \\
\hline Participation rate (\%) & 83.82 & & \\
\hline All patients with parkinsonism & 235 & 148 & 87 \\
\hline Primary parkinsonism & 170 & 137 & 33 \\
\hline Probable PD & 152 & 120 & 32 \\
\hline Possible PD & 4 & 4 & 0 \\
\hline PD overall & 156 & 124 & 32 \\
\hline Atypical parkinsonism & 14 & 13 & 1 \\
\hline MSA & 4 & 3 & 1 \\
\hline PSP & 6 & 6 & 0 \\
\hline Atypical parkinsonism not otherwise specified & 4 & 4 & 0 \\
\hline Secondary parkinsonism & 65 & 11 & 54 \\
\hline Drug-induced parkinsonism & 43 & 0 & 43 \\
\hline Parkinsonism following dementia & 5 & 0 & 5 \\
\hline Vascular parkinonism & 17 & 11 & 6 \\
\hline Patients without parkinsonism & & 54 & \\
\hline Non-parkinsonian tremor & & 46 & \\
\hline Other & & 8 & \\
\hline
\end{tabular}

